\title{
IMPLEMENTATION
}

AND EFFECTS

OF A SCHOOLWIDE

DATA-BASED

\section{DECISION MAKING}

INTERVENTION:

A LARGE-SCALE STUDY

Trynke Keuning \& Marieke van Geel 



\section{IMPLEMENTATION AND EFFECTS \\ OF A SCHOOLWIDE \\ DATA-BASED DECISION MAKING INTERVENTION: \\ A LARGE-SCALE STUDY}

Trynke Keuning

Marieke van Geel 
Samenstelling promotiecommissie

Voorzitter

Promotoren

Leden

Prof. dr. T.A.J. Toonen

Prof. dr. A.J. Visscher

Prof. dr. ir. J.-P. Fox

Prof. dr. R.J. Bosker

Prof. dr. P.J. den Brok

Prof. dr. T.J.H.M. Eggen

Prof. dr. S.E. McKenney

Prof. dr. K. van Veen

Prof. dr. ir. B.P. Veldkamp

ico

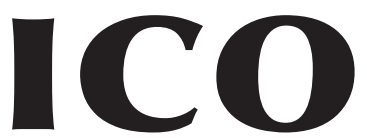

\begin{tabular}{|cc|}
\hline FSC & $\begin{array}{c}\text { MIX } \\
\text { Paper from } \\
\text { responsible sources } \\
\text { FSC }\end{array}$ \\
\hline www.fsc.org & C012854 \\
\hline
\end{tabular}

Druk: Gildeprint, Enschede

ISBN: 978-90-365-4119-0

DOI: 10.3990/1.9789036541190

Copyright (C) 2016, Keuning, Trynke \& Van Geel, Marieke 


\title{
IMPLEMENTATION AND EFFECTS \\ OF A SCHOOLWIDE \\ DATA-BASED DECISION MAKING INTERVENTION: \\ A LARGE-SCALE STUDY
}

\author{
PROEFSCHRIFT \\ ter verkrijging van de graad van doctor \\ aan de Universiteit Twente \\ op gezag van de Rector Magnificus \\ prof. dr. H. Brinksma, \\ volgens besluit van het College voor Promoties \\ in het openbaar te verdedigen \\ op vrijdag 8 juli 2016
}

om 14.45 uur

door

Marieke Johanna Maria van Geel

geboren op 25 oktober 1984

in Papendrecht om 16.45 uur

door

Trynke Keuning

geboren op 17 juli 1988

in Dokkum 
Dit proefschrift is goedgekeurd door de promotoren:

Prof. dr. A.J. Visscher

Prof. dr. ir. J.-P. Fox 


\section{Contents}

CHAPTER 1

INTRODUCTION

\section{CHAPTER 2}

ASSESSING THE EFFECTS OF A SCHOOLWIDE DATA-BASEd DECISION MAKING

INTERVENTION ON STUDENT ACHIEVEMENT GROWTH IN PRIMARY SCHOOLS

\section{CHAPTER 3}

THE EFFECTS OF A SCHOOLWIDE DATA-BASEd DeCISION MAKING INTERVENTION on ELEMENTARY SCHOOL'S StUdent ACHIEVEMENT GROWTH FOR MATHEMATICS AND SPELLING

\section{CHAPTER 4}

CHANGES IN EDUCATORS' DATA LITERACY DURING A DATA-BASED DECISION MAKING INTERVENTION

\section{CHAPTER 5}

THE DeVElopment of TeACHING QUALITY DURING A DATA-BASEd DeCISION MAKING INTERVENTION: A PRE-POST MULTILEVEL IRT APPROACH

\section{CHAPTER 6}

State TRANSitions in Educational LEAdERSHIP DURING A DATA-BASED

DECISION MAKING INTERVENTION

\section{CHAPTER 7}

DEVELOPMENT OF SCHOOlS' DATA CULTURE DURING A SCHOOLWIDE DATABASED DECISION MAKING INTERVENTION

\section{CHAPTER 8}

THE TRANSFormation of SCHOOls' SOCIAL NeTWORKS DURING A DATA-BASED DECISION MAKING REFORM

\section{CHAPTER 9}

WHY A DATA-BASED DECISION MAKING INTERVENTION WORKS IN SOME

SCHOOLS AND NOT IN OTHERS

\section{CHAPTER 10}


A DETAILED EXPLANATION OF THE EQUATIONS FOR EACH MODEL IN CHAPTER 2

\section{APPENDIX B}

DATA LITERACY TEST

\section{APPENDIX C}

AppendiX to Chapter 5: "The DeVelopment of TeACHING Quality DuRING A DBDM INTERVENTION: A PRE-POST MULTILEVEL IRT APPROACH"

\section{APPENDIX D}

QUESTIONNAIRE LEADERSHIP FOR DBDM

\section{APPENDIX E}

QUESTIONNAIRE DATA CULTURE

APPENDIX F

R SCRIPT OF THE MULTIVARIATE LINEAR ANALYSIS USING tHE CAR PACKAGE AND CONTRAST MATRICES TO TEST HYPOTHESES

APPENDIX G

259

Questionnaire to Measure Educators' AtTitude

APPENDIX H

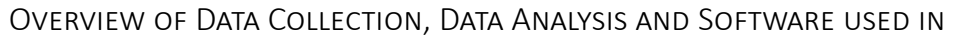

CHAPTER 9

REFERENCES 


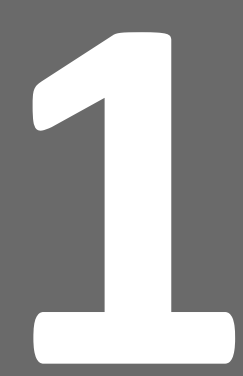

Introduction 
In many countries around the world, the systematic evaluation and analysis of student achievement data, and the subsequent linking of the results to classroom practice, to enhance student outcomes is becoming popular. In 2007, the results of international comparative studies like PIRLS 2006 (Mullis, Martin, Kennedy, \& Foy, 2007), PISA (OECD, 2007), and TIMSS (Martin, Mullis, \& Foy, 2008) showed that the Netherlands was one of the countries for which a decline in performance in the core subjects (mathematics and reading) was found. Therefore, 'data-based decision making' (DBDM) became a core theme in Dutch educational policy. The Dutch Inspectorate of Education qualified DBDM as the key to educational improvement (Inspectie van het Onderwijs, 2011). According to their own data, in schools in which DBDM was implemented more, student achievement was higher (Inspectie van het Onderwijs, 2010). During the last few years, a large number of Dutch primary schools have acknowledged the importance of DBDM, and have started to implement DBDM in order to improve student achievement in reading, language and/or mathematics. The Inspectorate uses a set of five indicators to determine to what extent schools apply DBDM in practice: 1) the school uses a coherent system of standardized tools and procedures for monitoring the performance and development of students; 2) teachers monitor and analyse students' progress in a systematic way; 3 ) the school evaluates the effects of educational care on a regular basis; 4) the school annually evaluates students' results; 5) the school evaluates the educational process on a regular basis (Inspectie van het Onderwijs, 2015).

Despite the ambition of the Dutch Ministry of Education of a 90 percent score in 2018 , the percentage of schools that score satisfactorily on all five indicators has remained constant around 30 percent over the last three years, (Inspectie van het Onderwijs, 2015). In order to achieve this goal, initiatives to promote data use and DBDM in primary schools are supported by the Dutch government. One of those initiatives is the 'Focus' intervention, developed by the University of Twente. This dissertation is aimed at evaluating the effects of this intervention on both student achievement as well as determining the characteristics of educators and schools that enhance the implementation of DBDM. Furthermore, we aim to identify the effect of the intervention on those characteristics.

In this chapter, the concept of DBDM is first defined, and scientific evidence for the components of DBDM is discussed. Next, evidence on the effectiveness of DBDM will be described as well as the challenges and opportunities to enhance DBDM. After the characteristics of the Focus intervention have been described, the research questions and the outline of this dissertation will be presented.

\section{A Definition of DBDM}

In today's society, data play an important role in informing decisions in all sectors of society, from commercial organizations to adjust their sales strategy based on the analysis of customer behavior, to hospitals evaluating their treatment effectiveness, and teachers adapting their instruction to well-defined student needs (Lai \& Schildkamp, 2013). Technological developments make it possible to collect, analyze, interpret and distribute data in an efficient and systematic way (Mandinach, 2012).

In education, the increasing interest in data use is twofold. On the one hand, there is the accountability context in which school leaders and teachers are held accountable for the quality of the education they provide (Lai \& Schildkamp, 2013). Data, such as student achievement scores on standardized tests, are used in a summative way for the purpose of accountability to external parties such as parents and (in the Netherlands) the Inspectorate 
of Education. On the other hand, there is a growing recognition that data should not only be used for compliance and accountability but also for continuous improvement (Lai \& Schildkamp, 2013; Mandinach, 2012; Kingston \& Nash, 2011). In that context, data use is seen as a way to inform teachers about students' needs, and to adapt and adjust instruction based on this information. School leaders can use data to base their school level decisions on (Lai \& Schildkamp, 2013). Mandinach (2012) argued that data use to inform instructional decisions is not new. Teachers collect information about their students all the time: they ask questions, observe students, and examine student work. Mostly, teachers process this information in their heads to determine how to proceed. However, this is not always done systematically. Due to technological developments, like the development of student monitoring systems, it is now possible to use data in a more systematic way. For example, these systems enable schools to compare student outcomes with previous performance, or to represent data in graphs and tables by retrieving those immediately from the system.

The use of data to enhance instruction and to improve achievement at the class, school and school board level ${ }^{1}$ is known as Data-Driven Decision Making (DDDM), or Data-Based Decision Making (DBDM). Ikemoto and Marsh (2007) use the following broad definition of DBDM: "teachers, principals, and administrators systematically collecting and analyzing data to guide a range of decisions to help improve the success of students and schools" (Ikemoto \& Marsh, 2007, p. 108). Visscher and Ehren (2011) distinguish three core components of DBDM: 1) evaluating the starting situation of students, student groups and the school as a whole; 2) defining goals; 3) choosing strategies to accomplish those goals. Together, these components are assumed to improve education and to lead to improved student achievement. Based on the three components, and the definition given by Ikemoto and Marsh (2007), the model for DBDM as used in this dissertation was developed (Figure 1).

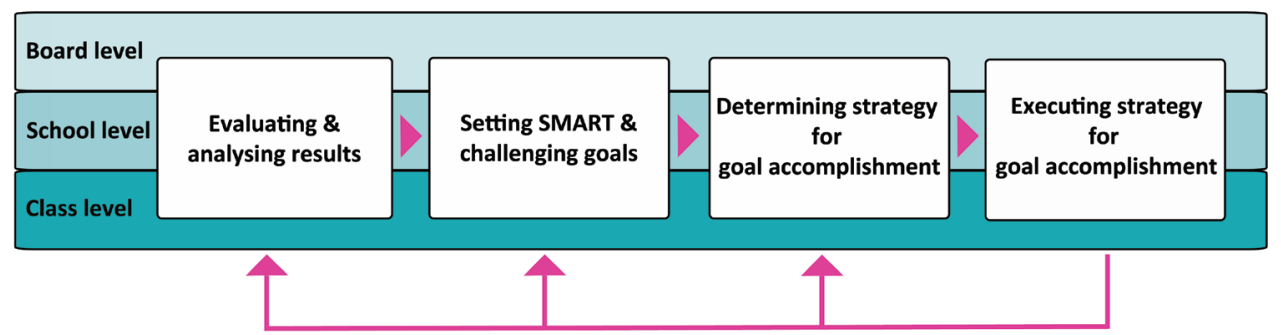

Figure 1. Schematic overview of DBDM

The four DBDM components and the relationships assumed between them will now be explained. At the class, school and board levels, student and school performance data are supposed to be analysed, and decisions are supposed to be based on the results of the analyses. Since the aim of DBDM is to systematically maximize student achievement of all students, the focus is explicitly on evaluating and analysing student performance data. Standardized test results are the starting point, but ideally additional information also is gathered because no single assessment can provide all the information necessary, to make informed decisions (Hamilton et al., 2009). Based on the analysis of the standardized test results, teachers come to understand their students' strengths and weaknesses, and they can

1 In the Netherlands, schools are not organized in districts, often there is one school board for several schools (approximately $8 \%$ of the schools are 'single schools' with one board, the other 7.000 schools belong to one of 569 boards) (PO-Raad, 2010) 
combine those insights with curriculum-based tests, classwork, homework and classroom observations, to determine students' instructional needs more in detail.

In the DBDM literature, the term 'decisions' implies a variety of actions that can be undertaken on the basis of data, such as: setting goals, adapting instruction, adapting the curriculum, improving policy and reallocating time and resources as necessary (Earl \& Katz, 2006; Hamilton et al., 2009; Ikemoto \& Marsh, 2007; Mandinach, Gummer, \& Muller, 2011). In the schematic representation of DBDM (Figure 1), 'decisions' are decomposed into 'setting goals' based on the data, and 'determining strategies' in order to achieve those goals.

The goals set and the strategies chosen for goal accomplishment vary according to the level of decision making. At the group level, teachers can use student performance data to differentiate instruction (Dunn, Airola, Lo, \& Garrison, 2013). Teachers first set goals in terms of desired achievement gains, or skill attainment. To accomplish those goals, they can (for example) decide to use a specific instructional strategy, or form a separate group of students to work on improving a specific skill. At the school and board levels, data can be used to highlight specific areas for improvement in the school(s), and the strategies chosen often comprise policy decisions, or (for example) the allocation of resources, or the modification of the curriculum.

The final step is to implement and execute the chosen strategies. As Bennett (2011), Anderson, Leithwood, and Strauss (2010) and others have argued, the effects of these implementation activities are closely related to the quality of the inferences drawn on the basis of the data, the chosen approach for addressing the identified problems, and the instructional expertise of those working in schools. If, for example teachers draw incorrect conclusions about students' learning needs, they are likely to implement a strategy that is unlikely to lead to the desired outcomes.

Since DBDM is intended to be implemented as a systematic approach, data are also supposed to be used for monitoring and evaluating the effects and outcomes of implemented strategies, for evaluating the extent to which goals have been achieved, and for making new data-informed decisions.

Additionally, DBDM takes place at all levels of education and it is important that support is consistent and well-coordinated across these levels (Anderson et al., 2010; Berends, Bodilly, \& Kirby, 2002; Desimone, 2002). The school board can play a crucial role in achieving and maintaining such consistency. Mandinach (2012) emphasized the importance of a vision, norms and goals at the board and the district level. An explicit vision should be aligned across all levels throughout the board or district, in order to set clear expectations for and purposes of data use.

\section{DBDM - Why Is It Supposed to Work?}

It is assumed that DBDM has a positive influence on student outcomes (Turner \& Coburn, 2012), as research has demonstrated positive effects of each separate component of DBDM.

The first component in the DBDM model is the use of performance data, to analyze and evaluate student results. This can be regarded as using performance feedback: student monitoring systems provide feedback to schools and teachers, in fact they reflect how students, teachers, and schools perform in comparison with the national average performance level, whether students' progress is adequate, and how much students master subject matter elements. The positive, performance improving effects of using feedback have 
been shown in several reviews and meta-analyses (Black \& Wiliam, 1998; Fuchs \& Fuchs, 1986; Hattie \& Timperley, 2007; Hattie, 2009; Kluger \& DeNisi, 1996). Studies investigating the use of performance feedback in an educational setting (for example Coe, 2002; Gray, 2002; Oláh, Lawrence, \& Riggan, 2010; Vanhoof, Verhaeghe, Van Petegem, \& Valcke, 2013), identified critical features of effective student performance feedback in schools. Among other things, the degree to which feedback recipients obtain an idea of how they can improve is very important. Information that one is underperforming without an idea of the cause of underperformance, and about how performance may be improved makes demotivation more likely than improving performance. Furthermore, a clear graphical representation of data is crucial for improving the understanding and use of performance feedback (Verhaeghe, 2011).

The second element of DBDM - setting SMART and challenging goals - also has proven effects on performance. Locke and Latham (2002), who developed their goal setting theory for various types of tasks, showed that setting challenging and SMART (Specific, Measurable, Attainable, Relevant, Time-bound) learning goals, or performance goals significantly helps in improving performance. Goal setting in combination with the use of feedback improves performance even more (Locke \& Latham, 2002). The mechanism that explains such goal setting effects is that difficult but attainable goals in general motivate people. Explicit SMART goals also make people focus their activities more (leading to less variation in the definition of goals, reducing activities that do not contribute to goal accomplishment, and increasing time on task), and they promote the search for and utilization of task-relevant knowledge leading to improved performance (Morisano \& Locke, 2013).

The third and fourth element of DBDM concern choosing and executing a strategy for goal accomplishment. Although the type of strategy chosen is dependent on the level of decision-making (group, school, or board), in general all strategies are aimed at improving instruction, and consequently at improving student achievement. It is generally accepted that what teachers do in the classroom is the most important malleable factor influencing student performance (Hanushek, 2011; Hattie, 2009; Nye, Konstantopoulos, \& Hedges, 2004). Therefore, it makes sense to search for ways to improve the quality of teacher behavior, since ultimately it is the quality and the execution of the chosen instructional strategies that is decisive for increasing student achievement.

\section{Scientific Evidence for DBDM - What Do We Know?}

As described in the previous section, studies into the effects of each of the core components of DBDM led to encouraging results. Although DBDM 'as a package of those components' is therefore regarded as a promising approach for improving student achievement, the effects of DBDM interventions on student achievement so far were mostly insignificant (Henderson, Petrosiono, Guckenburg, \& Hamilton, 2007; Quint, Sepanik, \& Smith, 2008; Ritzema, 2015), or small (Campbell \& Levin, 2008; Carlson, Borman, \& Robinson, 2011; Konstantopoulos, Miller, \& van der Ploeg, 2013; Lai \& McNaughton, 2013; May \& Robinson, 2007). This does not necessarily imply that DBDM in education is not effective, but rather suggests that more research is needed on how data use can reach its full potential (Kaufman, Graham, Picciano, Popham, \& Wiley, 2014). In order to develop a DBDM intervention that might reach this full potential, those factors that are assumed to support the implementation of DBDM should be taken into account. 


\section{Factors Influencing the Implementation of DBDM}

Although studies on the relationships between DBDM and student outcomes are scarce and inconclusive, numerous studies in the field of DBDM were dedicated to determining the factors that support the implementation of DBDM effectively. Based on Schildkamp and Lai (2013), Mandinach (2012), Ikemoto and Marsh (2007), and Visscher and Ehren (2011), several factors related to the implementation of DBDM can be distinguished. Although this is not an exhaustive list, the factors described below are considered the most relevant ones for enhancing the implementation of all four DBDM components at all levels of education. These factors can be divided into three categories: 1) resources; 2) educator characteristics; and 3) school organizational characteristics.

\section{Resources}

a) The availability of assessment tools for collecting data, especially on student progress. These assessment tools can vary from teachers' daily observations to high stake standardized tests, but their overall aim is to "inform teachers about the understanding of their students in order to inform subsequent teaching" (Supovitz, 2012 , p. 2). Since the data collected by means of assessments is used to base decisions on, the quality of these assessment tools is of great importance.

b) Technological tools to make the data accessible and interpretable. To handle the increasing amount of (complex) data, technological tools are needed to help educators collect, analyze, interpret and report data in meaningful ways (Bernhardt, 2005; Mandinach, 2012). A student monitoring system (SMS) is an example of such a tool: in a SMS a teacher can store data, visualize those data in graphs and tables, and compare the results with previous results, or national benchmarks (Kamphuis \& Moelands, 2000). These technological tools need to be easily accessible, deliver results timely, and be user-friendly (Datnow, Park, \& Wohlstetter, 2007; Ikemoto \& Marsh, 2007).

c) Time to implement $D B D M$ is important for two reasons. First, developing DBDM implies that school teams have to acquire new knowledge and skills. This process takes time and will include a period of practicing and gradually optimizing DBDM (Desimone, 2002). Second, DBDM implies new activities: time must be scheduled to analyze and discuss data, to set goals, and to determine and execute the strategy for goal accomplishment (Kirkup, Sizmur, Sturman, \& Lewis, 2005; Visscher \& Ehren, 2011).

\section{Educator Characteristics}

d) Knowledge and skills. Implementing DBDM requires knowledge and skills with regard to the components of DBDM: 1) analyzing an interpreting data (also known as 'data literacy') (Bennett, 2011; Mandinach, Honey, \& Light, 2006); 2) setting SMART and challenging goals (Visscher \& Ehren, 2011); 3) choosing effective strategies to meet those goals and; 4) executing these strategies in practice (Anderson et al., 2010; Visscher \& Ehren, 2011).

e) Attitudes towards DBDM. A positive attitude is regarded as essential, because school teams, including the school leader, need to be motivated to implement DBDM in their school (Hammond \& Yeshanew, 2007; Lai \& Schildkamp, 2013; Reeves \& Burt, 2006; Schildkamp \& Ehren, 2013; Schildkamp, Karbautzki, \& Vanhoof, 2014; Vanhoof, Van Petegem, \& De Maeyer, 2009). 


\section{School Organizational Characteristics}

f) Leadership for $D B D M$. In addition to the responsibility for fulfilling the practical preconditions for DBDM (e.g. selecting a proper student monitoring system, and providing time for DBDM activities) (Datnow \& Stringfield, 2011; Ikemoto \& Marsh, 2007; Lai \& Schildkamp, 2013) a school leader is assumed to have a key role in influencing teachers' attitude towards DBDM, and in creating a data culture by promoting clear visions and norms to DBDM (Coburn \& Turner, 2011; Hamilton et al., 2009; Marsh, 2012).

g) Data culture. A culture that is achievement oriented and fosters data use is regarded essential for implementing and sustaining DBDM (Ingram, Louis, \& Schroeder, 2004; Mandinach, 2012; Wayman, Midgley, \& Stringfield, 2006). According to Lai and Schildkamp (2013), DBDM is best implemented in schools in which team members feel the need to critically look at data, to reflect on their own functioning, and who are open to changing their practice if the data reveal a need to do so. However, it is not always easy to constantly reflect on one's own functioning without feeling threatened (Marsh, 2012). A climate of trust is therefore essential in establishing a data culture within schools (Ikemoto \& Marsh, 2007; Marsh, 2012; Visscher \& Ehren, 2011). In such a culture, data is not used to judge but to support improvement.

h) Collaboration. In schools with strong collaboration patterns, teachers may have good access to the knowledge and skills for analyzing data; have the opportunity to discuss challenging performance goals; and share effective teaching strategies to achieve those goals (Ingram et al., 2004; Lai \& McNaughton, 2009; Schildkamp \& Lai, 2013).

\section{The DBDM Intervention}

As it was described in the previous, DBDM is regarded a promising strategy to enhance student achievement, and was made a core theme in Dutch educational policy. It is acknowledged that professional development activities are needed, for example to acquire the knowledge and skills required for successfully implementing DBDM (Mandinach, 2012; Timperley, 2008). The Dutch government therefore supported professionalization initiatives, to promote data use and DBDM in schools.

One of those initiatives was the 'Focus' intervention, developed at the University of Twente. After a pilot study, and the first Focus project ran in 43 schools in the Netherlands (Staman, Visscher, \& Luyten, 2013), the intervention was optimized on the basis of experiences and new insights, and was implemented as the 'Focus II project' during the school years 2011-2013, and the 'Focus III project' in the school years 2012-2014. The Focus II and Focus III projects are the main focus of this dissertation. In the remainder of this dissertation, the term 'Focus', refers to both Focus II and Focus III, unless explicitly stated otherwise.

The Focus project was a two year training course for entire primary school teams, aimed at as a school acquiring the knowledge and skills for DBDM, and implementing and sustaining DBDM 'as a package' (as depicted in Figure 1) including all four components, at all levels of the school organization. In order to promote the successful implementation of Focus, the factors influencing the implementation of DBDM (as described in the previous) were taken into account. The design of the intervention was based on the literature on effective professional development. 
In the following sections, first the design of the Focus intervention is related to the professional development literature. In the section thereafter, the content of the Focus intervention is described and finally, some differences between the two Focus projects are highlighted.

\section{Design of the Intervention}

The design of the Focus intervention was based on insights from the professional development literature. Three core aspects of the intervention design were: collective participation, time, and the use of protocols and documents.

Collective participation. All teachers and members of the school management team (such as the school leader and the academic coach), participated in the intervention, since collective participation is generally found to be positively correlated with active participation in professional development activities (Garet, Porter, Desimone, Birman, \& Yoon, 2001; Lumpe, 2007). Van Veen, Zwart, and Meirink (2011), and Timperley (2008) argued that the interaction with and collaboration between colleagues is important for implementing and mastering an innovation.

Time. It takes time to learn and change, and therefore 'time' is a structural feature of professional development in two ways: the time span over which the intervention is spread, and the number of contact hours (Birman, Desimone, Porter, \& Garet, 2000; Desimone, Porter, Garet, Yoon, \& Birman, 2002; Garet et al., 2001).

In Focus, the time span of the intervention was two subsequent school years: a total of 22 months. There were 14 contact moments (each of approximately 4 hours), and additional to these contact moments, participants were supposed to apply what they have learned in practice, for instance by carrying out analyses, developing instructional plans, and, finally, adapting their instruction. Teachers gradually practiced and implemented what they had learned, which is also an important aspect of effective professional development activities (Timperley, 2008; Van Veen et al., 2011).

The use of protocols and documents. Comprehensive school reform is more easy and faster for externally developed reform designs than for locally developed designs (by districts, or schools). The reason for this is that they often provide detailed implementation guidelines (Desimone, 2002). On the other hand, staff seems to support reform better when they are actively engaged in co-constructing the changes in their schools in such a way that those changes fit their local context (Datnow, Hubbard, \& Mehan, 1998). Timperley (2008) stressed that external experts are more effective when they involve teachers in discussing and developing understandings that are meaningful in their own context.

In Focus, schools were initially provided with the same protocols, documents and planning aids, to help them incorporate DBDM in their organization and practice. During training sessions, these protocols and documents however were discussed and adapted to fit the local school context.

\section{Content of the Intervention}

The intervention was aimed at acquiring the knowledge and skills for DBDM by school staff, and at implementing and sustaining DBDM in the school organization (and at the school board level, if applicable ${ }^{2}$ ), by following the DBDM cycle as depicted in Figure 1 . In Table 1 an overview of the intervention meetings is provided. The first five meetings (meeting 1.1

2 The board level was included in the intervention for boards of which the majority of schools participated in the intervention. School boards were stimulated to support and encourage schools in implementing DBDM; implementing DBDM at the board level was not the main focus of this intervention. 
Table 1. Projects overview

\begin{tabular}{|c|c|c|c|c|}
\hline & & Focus II & & Focus III \\
\hline \multirow[t]{8}{*}{$\overline{\mathrm{Y} 1}$} & $\begin{array}{l}\text { School leader } \\
\text { / school board } \\
\text { meeting }\end{array}$ & $\begin{array}{l}\text { Fulfilling practical } \\
\text { preconditions and } \\
\text { stressing the importance } \\
\text { of the role of the school } \\
\text { leader/school board }\end{array}$ & $\begin{array}{l}\text { School leader } \\
\text { / school board } \\
\text { meeting }\end{array}$ & $\begin{array}{l}\text { Fulfilling practical } \\
\text { preconditions and } \\
\text { stressing the importance } \\
\text { of the role of the school } \\
\text { leader/school board }\end{array}$ \\
\hline & Team meeting & $\begin{array}{l}\text { Analyzing test score } \\
\text { data from the student } \\
\text { monitoring system }\end{array}$ & Team meeting & $\begin{array}{l}\text { Analyzing test score } \\
\text { data from the student } \\
\text { monitoring system }\end{array}$ \\
\hline & Team meeting & $\begin{array}{l}\text { Subject matter content - } \\
\text { curriculum }\end{array}$ & Team meeting & $\begin{array}{l}\text { Subject matter content - } \\
\text { curriculum }\end{array}$ \\
\hline & & $\begin{array}{l}\text { Individual diagnosis of } \\
\text { students' learning needs }\end{array}$ & & $\begin{array}{l}\text { Individual diagnosis of } \\
\text { students' learning needs }\end{array}$ \\
\hline & Team meeting & $\begin{array}{l}\text { Goal setting and } \\
\text { developing instructional } \\
\text { plans }\end{array}$ & Team meeting & $\begin{array}{l}\text { Goal setting and } \\
\text { developing instructional } \\
\text { plans }\end{array}$ \\
\hline & Team meeting & $\begin{array}{l}\text { Instructional plans in } \\
\text { practice }\end{array}$ & Team meeting & $\begin{array}{l}\text { Instructional plans in } \\
\text { practice }\end{array}$ \\
\hline & & $\begin{array}{l}\text { Monitoring and adjusting } \\
\text { instructional plans based } \\
\text { on test data from content } \\
\text { mastery tests and daily } \\
\text { work in class }\end{array}$ & & $\begin{array}{l}\text { Monitoring and adjusting } \\
\text { instructional plans based } \\
\text { on test data from content } \\
\text { mastery tests and daily } \\
\text { work in class }\end{array}$ \\
\hline & $\begin{array}{l}\text { School leader } \\
\text { /school board } \\
\text { meeting }\end{array}$ & $\begin{array}{l}\text { Discussing progress and } \\
\text { the goals for the next } \\
\text { period (trainer, school } \\
\text { leader and school board) }\end{array}$ & $\begin{array}{l}\text { School leader } \\
\text { / school board } \\
\text { meeting }\end{array}$ & $\begin{array}{l}\text { Discussing progress and } \\
\text { the goals for the next } \\
\text { period (trainer, school } \\
\text { leader and school board) }\end{array}$ \\
\hline 1.5 & Team meeting & $\begin{array}{l}\text { Team meeting: evaluating } \\
\text { standardized test } \\
\text { performance data } \\
\text { Additional: math content } \\
\text { and curriculum }\end{array}$ & Team meeting & $\begin{array}{l}\text { Team meeting: evaluating } \\
\text { standardized test } \\
\text { performance data }\end{array}$ \\
\hline \multirow[t]{2}{*}{1.6} & Team meeting & $\begin{array}{l}\text { Collaboration in the } \\
\text { school: how to learn } \\
\text { from each other by using } \\
\text { classroom observations }\end{array}$ & Team meeting & $\begin{array}{l}\text { Collaboration in the } \\
\text { school: how to learn } \\
\text { from each other by using } \\
\text { classroom observations }\end{array}$ \\
\hline & $\begin{array}{l}\text { School leader } \\
\text { /school board } \\
\text { meeting }\end{array}$ & $\begin{array}{l}\text { Discussing progress and } \\
\text { goals for the next period } \\
\text { (trainer, school leader and } \\
\text { school board) }\end{array}$ & $\begin{array}{l}\text { School leader } \\
\text { /school board } \\
\text { meeting }\end{array}$ & $\begin{array}{l}\text { Discussing progress and } \\
\text { goals for the next period } \\
\text { (trainer, school leader and } \\
\text { school board) }\end{array}$ \\
\hline 1.7 & Team meeting & $\begin{array}{l}\text { Team meeting: evaluating } \\
\text { standardized test } \\
\text { performance data }\end{array}$ & Team meeting & $\begin{array}{l}\text { Team meeting: evaluating } \\
\text { standardized test } \\
\text { performance data }\end{array}$ \\
\hline
\end{tabular}




\begin{tabular}{|c|c|c|c|c|c|}
\hline & & & Focus II & & Focus III \\
\hline \multirow[t]{5}{*}{$\bar{Y} 2$} & 2.1 & Team meeting & $\begin{array}{l}\text { Option 1: Continue with } \\
\text { math: based on issues } \\
\text { raised by school } \\
\text { Option 2: New subject: } \\
\text { tests and analysis for new } \\
\text { subject }\end{array}$ & Team meeting & $\begin{array}{l}\text { Option 1: Continue with } \\
\text { the same subject: based } \\
\text { on issues raised by school } \\
\text { Option 2: New subject: } \\
\text { tests and analysis for } \\
\text { new subject, content and } \\
\text { curriculum }\end{array}$ \\
\hline & 2.2 & Team meeting & $\begin{array}{l}\text { Option 1: Continue for } \\
\text { math: based on issues } \\
\text { raised by the school } \\
\text { Option 2: New subject: } \\
\text { content and curriculum }\end{array}$ & $\begin{array}{l}\text { Classroom } \\
\text { observations }\end{array}$ & Classroom observations \\
\hline & 2.3 & Team meeting & $\begin{array}{l}\text { Team meeting: evaluating } \\
\text { standardized test } \\
\text { performance data } \\
\text { Optional: adding another } \\
\text { subject, tests and analysis } \\
\text { for new subject }\end{array}$ & Team meeting & $\begin{array}{l}\text { Team meeting: evaluating } \\
\text { standardized test } \\
\text { performance data } \\
\text { Optional: adding another } \\
\text { subject, tests and analysis } \\
\text { for new subject }\end{array}$ \\
\hline & 2.4 & $\begin{array}{l}\text { Classroom } \\
\text { observations }\end{array}$ & Classroom observations & $\begin{array}{l}\text { Classroom } \\
\text { observations }\end{array}$ & Classroom observations \\
\hline & 2.5 & Team meeting & $\begin{array}{l}\text { Team meeting: evaluating } \\
\text { standardized test } \\
\text { performance data. }\end{array}$ & Team meeting & $\begin{array}{l}\text { Team meeting: evaluating } \\
\text { standardized test } \\
\text { performance data. }\end{array}$ \\
\hline
\end{tabular}


to 1.5) were therefore spent on the knowledge and skills required to complete each of the following steps: analyzing and interpreting student achievement data, diagnosis of students' learning needs, setting goals, determining a strategy to accomplish these goals, and, finally, completing the cycle by evaluating student achievement, and discussing the results of those evaluations in a team meeting during meeting 1.5. The evaluation of the student results also was the first step of the new cycle for DBDM.

During meeting 1.5, it was stressed by the trainers, school leaders and other participants that data should be used to support improvement, and not to judge. This was meant to contribute to a culture of trust and collaboration, a school culture in which the school team as a whole felt the responsibility for students' performance. Meeting 1.6 was focused more specifically at collaboration among team members by observing each other's lessons, either to learn from the colleague visited, or providing him/her with feedback on specific topics. Finally, in meeting 1.7, like in meeting 1.5, student outcomes were discussed and plans were made for the second school year.

The five meetings in the second year (meeting 2.1 to 2.5) were aimed at internalizing, sustaining and broadening the scope of DBDM within the school, and at supporting participants with carrying out their decisions in practice, for example, by means of coaching sessions in which trainers were observing teachers, and in which they provided teachers with feedback. During the team meetings, attention was paid to issues that were raised by the school.

Next to the team meetings, the trainers met with school leaders and school boards twice a year, to discuss their role in the process, the school's progress, and the goals to be set for the upcoming period. During these meetings, the importance of encouragement and support of teachers (by school leaders and school boards) was stressed.

\section{Differences between Focus II and Focus III}

The two projects differed with regard to the subject for which DBDM was introduced first. All schools participating in the Focus II project started with implementing DBDM for mathematics. Schools participating in Focus III could choose to start with mathematics, spelling, vocabulary, or reading. After the first intervention year, schools in both projects either chose to continue with DBDM for the subject they started with, or to broaden the scope of DBDM to another subject. Halfway the second intervention year, schools could again choose to continue, or to broaden the scope of DBDM.

Another difference between the two interventions was the amount of time spent on subject matter content. Meeting 1.5 in the first year of Focus II lasted eight hours, compared to four for Focus III; these four extra hours were dedicated to subject matter content and curriculum matters. The second meeting in the second intervention year for Focus II was a team meeting about either issues raised by the school (in the case of a continuation of DBDM for mathematics), or subject matter content and curricular topics of the newly introduced subject. In Focus III, this time was spent on extra classroom observations.

Because of the large overlap between the two intervention projects, the research data from both projects are combined in this joint $\mathrm{PhD}$ thesis. This enabled us to investigate a broad array of factors related to the implementation, and the effects of DBDM. Marieke van Geel was responsible for the coordination of the Focus II project, Trynke Keuning was responsible for the coordination of the Focus III project. Each of the authors of this thesis is the main author of four chapters in this dissertation (see Table 2).

Figure 2 presents the time line of the data collection prior to and during the 
intervention. Note that Focus II started in school year 2011-2012 and that Focus III started one year later in school year 2012-2013. Similar data was collected within the two projects, except for information about teaching quality using student perception data, and information about collaboration patterns, which was only available in the Focus III project.

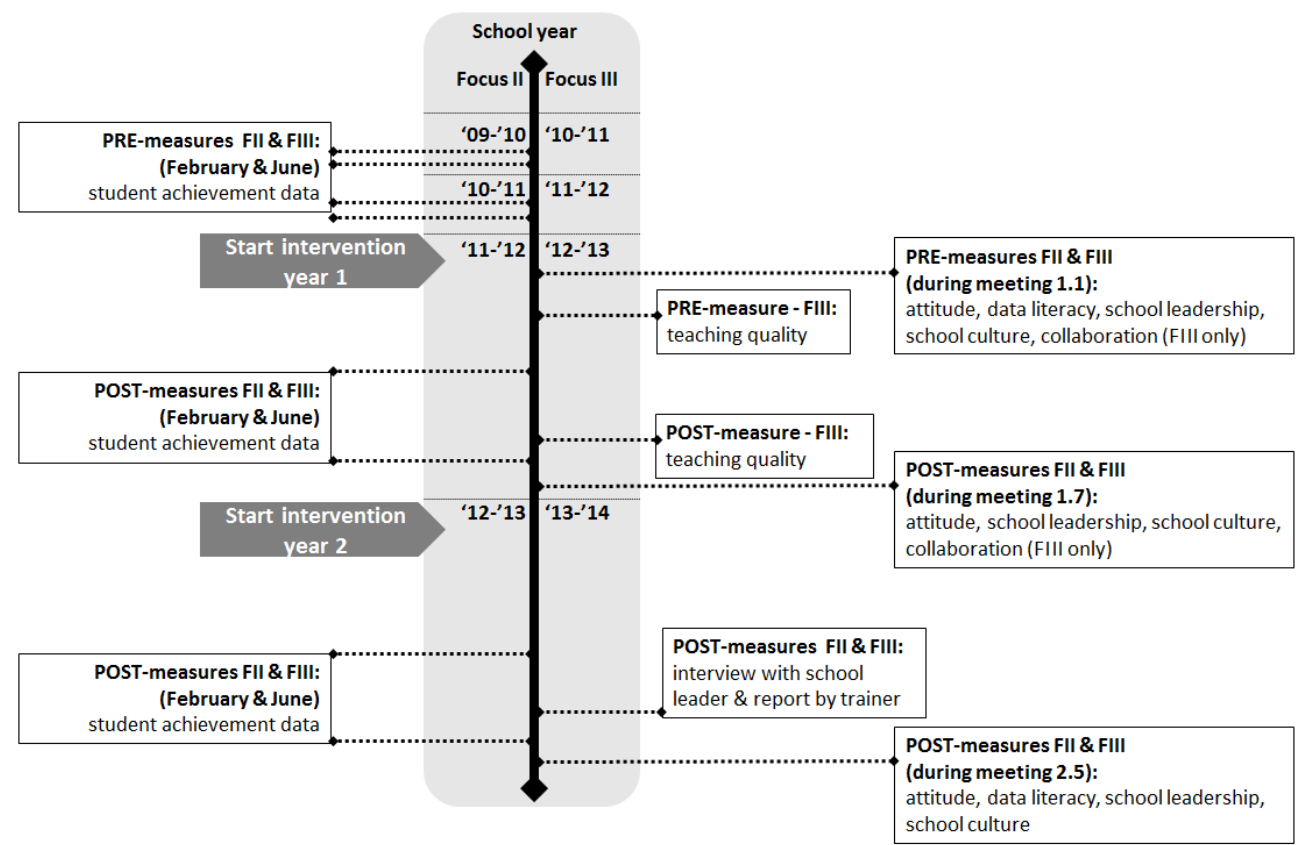

Figure 2. Time line data collection Focus II (FII) and Focus III (FIII) 


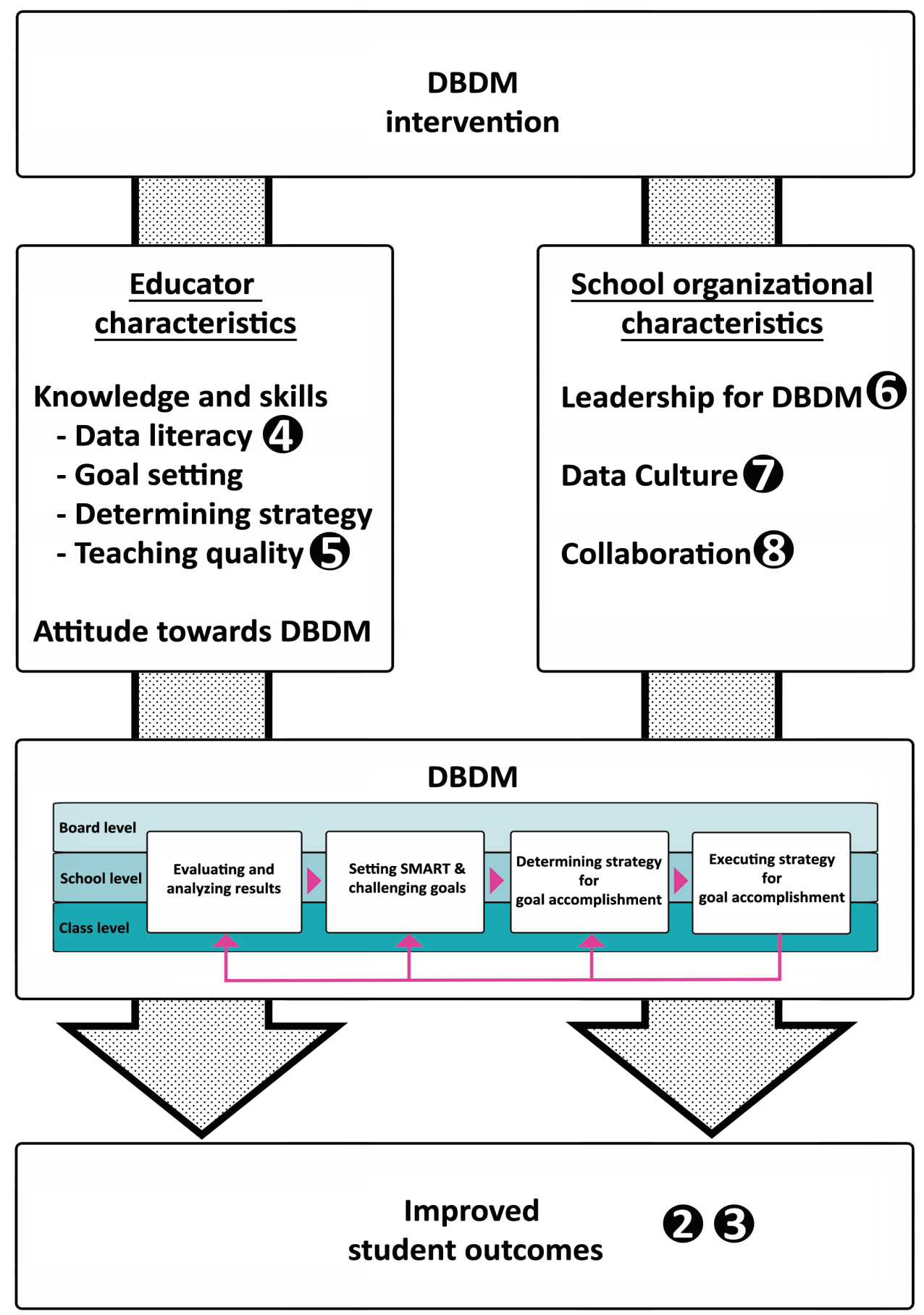

Figure 3. Conceptual model of this dissertation 


\section{Outline of this Dissertation}

Figure 3 presents a graphical overview of the conceptual model of this dissertation. As discussed in the previous, the intervention was primarily aimed at implementing DBDM and, by that, improve student outcomes. The main research question of this joint PhD dissertation is: has the DBDM intervention caused improved growth in student achievement? As shown in Figure 3 (indicated by the chapter numbers), this research question will be answered in two separate chapters for the two different Focus projects. For chapter 2 student achievement for mathematics was analyzed for 53 schools for the Focus II project. For chapter 3 (Focus III) student achievement in mathematics and spelling was analyzed for 40 schools.

In order to enhance DBDM implementation, educator and school organizational characteristics were taken into account. In chapters 4 and 5, data literacy and teaching quality are addressed in order to answer another research question: how do educator characteristics related to the implementation of DBDM change during the DBDM intervention? In chapter 4, the focus is on changes in educators' data literacy. In chapter 5 , changes in teaching quality as perceived by students are analyzed and discussed. For both these chapters, pre-post multivariate multilevel IRT analyses were conducted.

Chapters 6, 7 and 8 are focused on school organizational factors: leadership for DBDM, schools' data cultures, and school internal collaboration, to answer the research question: how do school organizational characteristics related to the implementation of $D B D M$ change during the DBDM intervention? For chapter 6 and 7, questionnaires to measure perceptions with regard to the school leader (chapter 6) and school data culture (chapter 7) were administered to all participating team members at the start, during, and at the end of the intervention. These perceptions were analyzed by means of multilevel latent class analysis, and multi-state modelling to investigate changes in leadership and schools' data cultures during the intervention. Collaboration was identified using social network measures (density, reciprocity, and centralization), and changes were modelled by means of multivariate linear analysis.

In order to assess the relationships between on the one hand the factors assumed to influence the implementation of DBDM, and on the other hand the actual intervention effects on student achievement, in chapter 9 an explorative study was conducted, to evaluate the differences between ten schools in which the intervention had the largest effects on student achievement with ten schools in which the intervention had the smallest effects on student achievement.

It should be noted that the required resources for DBDM implementation described earlier (the availability of assessment tools, technological tools to make data accessible and interpretable, and time to implement DBDM) were not included in the conceptual model of this dissertation as depicted in Figure 3. As far as assessment tools and technological tools are concerned, in the Dutch context a student monitoring system (SMS) has been developed by the Central Institute of Test Development (Kamphuis \& Moelands, 2000). This system includes a coherent set of tests (available for the core subjects: mathematics, reading, spelling and vocabulary) for the longitudinal assessment of students' achievement throughout all grades of primary education. The test results are converted into an ability scale for each subject so that student progress can be monitored over grades and school years (Kamphuis \& Moelands, 2000).

To process the collected test data, tools are required to help educators organize, analyze, interpret and report test data in meaningful ways (Bernhardt, 2005; Mandinach, 
Table 2. Dissertation overview

\begin{tabular}{|c|c|c|c|c|c|c|}
\hline Chapter & Main focus & Data analysis & Source & FII & FIII & $\begin{array}{l}\text { First } \\
\text { author }\end{array}$ \\
\hline \multicolumn{7}{|c|}{ Has the DBDM intervention caused improved growth in student achievement? } \\
\hline 2 & $\begin{array}{l}\text { Student } \\
\text { achievement } \\
\text { growth for } \\
\text { mathematics }\end{array}$ & $\begin{array}{l}\text { Linear mixed } \\
\text { models }\end{array}$ & $\begin{array}{l}\text { Students in grade } \\
1 \text { to } 6\end{array}$ & $\checkmark$ & & $\begin{array}{l}\text { Marieke } \\
\text { van Geel }\end{array}$ \\
\hline 3 & $\begin{array}{l}\text { Student } \\
\text { achievement } \\
\text { growth for } \\
\text { mathematics and } \\
\text { spelling }\end{array}$ & $\begin{array}{l}\text { Linear mixed } \\
\text { models }\end{array}$ & $\begin{array}{l}\text { Students in grade } \\
1 \text { to } 6\end{array}$ & & $\checkmark$ & $\begin{array}{l}\text { Trynke } \\
\text { Keuning }\end{array}$ \\
\hline \multicolumn{7}{|c|}{$\begin{array}{l}\text { How do educator characteristics related to the implementation of DBDM change during the DBDM } \\
\text { intervention? }\end{array}$} \\
\hline 4 & Data literacy & $\begin{array}{l}\text { Pre-post } \\
\text { multivariate } \\
\text { multilevel IRTa }\end{array}$ & $\begin{array}{l}\text { All participating } \\
\text { team members }\end{array}$ & $\checkmark$ & $\checkmark$ & $\begin{array}{l}\text { Marieke } \\
\text { van Geel }\end{array}$ \\
\hline 5 & Teaching quality & $\begin{array}{l}\text { Pre-post } \\
\text { multivariate } \\
\text { multilevel IRT }\end{array}$ & $\begin{array}{l}\text { Students in grade } \\
2 \text { to } 6\end{array}$ & & $\checkmark$ & $\begin{array}{l}\text { Trynke } \\
\text { Keuning }\end{array}$ \\
\hline
\end{tabular}

How do school organizational characteristics related to the implementation of DBDM change during the DBDM intervention?

\begin{tabular}{|c|c|c|c|c|c|c|}
\hline 6 & $\begin{array}{l}\text { Educational } \\
\text { leadership }\end{array}$ & $M L L C A^{b}$ and $M^{\prime} M^{c}$ & $\begin{array}{l}\text { All participating } \\
\text { team members, } \\
\text { except for the } \\
\text { school leaders }\end{array}$ & $\checkmark$ & $\checkmark$ & $\begin{array}{l}\text { Marieke } \\
\text { van Geel }\end{array}$ \\
\hline 7 & Data culture & ML LCA and MSM & $\begin{array}{l}\text { All participating } \\
\text { team members }\end{array}$ & $\checkmark$ & $\checkmark$ & $\begin{array}{l}\text { Marieke } \\
\text { van Geel }\end{array}$ \\
\hline 8 & Collaboration & $\begin{array}{l}\text { Social network } \\
\text { analysis } \\
\text { Multivariate linear } \\
\text { analysis }\end{array}$ & $\begin{array}{l}\text { All participating } \\
\text { team members }\end{array}$ & & $\checkmark$ & $\begin{array}{l}\text { Trynke } \\
\text { Keuning }\end{array}$ \\
\hline
\end{tabular}

What differentiates schools that benefit from the DBDM intervention from those that do not benefit from the intervention?

\begin{tabular}{|c|c|c|c|c|}
\hline $\begin{array}{l}\text { Identifying critical } \\
\text { factors for DBDM } \\
\text { implementation }\end{array}$ & Mixed methods & $\begin{array}{l}10 \text { strong-effect } \\
\text { schools and } 10 \text { no- } \\
\text { effect schools }\end{array}$ & $\checkmark$ & $\checkmark$ \\
\hline
\end{tabular}

Note. ${ }^{a}$ Item Response Theory (IRT), ${ }^{b}$ Multilevel Latent Class Analysis (ML LCA), ${ }^{c}$ Multi-State Modelling (MSM) 
2012). Student monitoring system software is an example of such a technical tool. After taking a test, teachers can store the student performance data in the SMS-software. Graphs and tables representing various aspects of student performance can then immediately be retrieved from the system, and with the SMS-software it also is possible to compare the scores of students with national benchmarks. The use of these standardized SMS tests and the registration in SMS software is common practice in the Netherlands, in other words, the availability of assessment tools and technological tools was not a concern.

Finally, 'time to implement DBDM' was not included in the conceptual model either, since this DBDM precondition was also met due to participating in the intervention. For example, teachers need time to conduct the analyses in the student monitoring system, and this time was provided during the first meeting. The same holds for, for example, time to discuss student achievement, performance goals, or instructional strategies.

In Table 2, an overview is presented per chapter of the research question, the topic studied, and the data analysis method used. Furthermore, the first author of each chapter is mentioned in order to identify the contribution of each author to this joint PhD thesis. Both authors take full responsibility for this introductory chapter as well as for the general conclusion (chapter 10), and the coherence of the thesis as a whole.

All chapters in this dissertation can be regarded as separate papers, which were submitted as articles to scientific journals. For each chapter it is indicated by/to which journal the article based on the chapter was accepted or submitted. Furthermore, since all chapters are related to the same intervention, there is some overlap between chapters. 


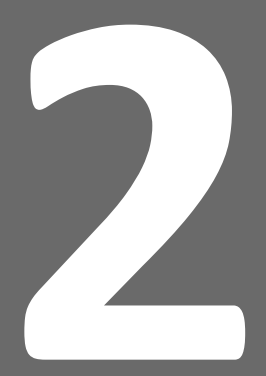

\section{Assessing the Effects of a Schoolwide Data-Based}

\section{Decision Making Intervention} on Student Achievement Growth in Primary Schools

This chapter is a modified version of the article:

Van Geel, M., Keuning, T., Visscher, A. J., \& Fox, J.-P. (2016). Assessing the Effects of a Schoolwide Data-Based Decision Making Intervention on Student Achievement Growth in Primary Schools. American Educational Research Journal, 53(2), 360-394. http://doi.org/10.3102/0002831216637346 


\section{Abstract}

Background. Despite growing international interest in the use of data to improve education, few studies examining the effects on student achievement are yet available.

Purpose. In the present study, the effects of a two-year data-based decision making intervention on student achievement growth were investigated.

Method. Fifty-three primary schools participated in the project, and student achievement data were collected over the two years before and two years during the intervention. Linear mixed models were used to analyze the differential effect of data use on student achievement.

Findings. A positive mean intervention effect was estimated, with an average effect of approximately one extra month of schooling. Furthermore, the results suggest that the intervention especially significantly improved the performances of students in low-SES schools. 
Today, data play an important role in informing decisions in all sectors of society: from commercial organizations adjusting their sales strategy based on the analysis of customer behavior, to hospitals evaluating their treatment effectiveness, and teachers adapting their instruction to well-defined student needs (Lai \& Schildkamp, 2013). In education, there is a growing emphasis on the use of data to base decisions on, assuming that this will lead to increased student achievement. Although only a few studies provide empirical evidence for the effect of data-based decision making (DBDM) on the achievement of students, there is considerable empirical evidence for the elements DBDM can be decomposed into, such as the impact of feedback, setting goals, and improving instructional quality.

In line with an increasing interest all over the world, the government in the Netherlands promotes the use of data to improve education. At the University of Twente, an intervention aimed at data-based decision making has been developed. A multiple singlesubject design was used to investigate the effect of this DBDM intervention on student achievement growth, and to explore patterns in DBDM effectiveness based on background variables at both the school and the student levels.

\section{Theoretical Framework}

\section{The Background of DBDM}

The increasing interest in data use in education is twofold. On the one hand, there is the accountability context in which school leaders and teachers are held accountable for the quality of the education they provide (Lai \& Schildkamp, 2013). Data, such as student achievement scores on standardized tests, are used in a summative way for the purpose of accountability to external parties such as parents and (in the Netherlands) the Inspectorate of Education. On the other hand, there is a growing recognition that data should not only be used for compliance and accountability but also for continuous improvement (Lai \& Schildkamp, 2013; Mandinach, 2012; Kingston \& Nash, 2011). In that context, data use is seen as a way to inform teachers about students' needs, and to adapt and adjust instruction based on such information. School leaders can use data as the basis for their decisions at the school level (Lai \& Schildkamp, 2013). Although there is growing emphasis on data use, Mandinach (2012) argues that data use to inform instructional decisions is nothing new. Teachers collect information about their students all the time: they ask questions, observe students, and examine students' work. Mostly, teachers process this information to help them make informed decisions. However, this may not always be done systematically. Technological developments enable educators to collect, analyze, interpret and distribute data in increasingly efficient and systematic ways (Mandinach, 2012). For example, a student monitoring system allows schools to monitor students' progress throughout their entire school careers (Kamphuis \& Moelands, 2000). In such a system, student achievement data can be easily stored, manipulated and retrieved. Furthermore, data can be represented in such a way that the data are easy to interpret (e.g. in graphs and growth-models). Moreover, the increased use of national standardized tests makes it possible to compare student performance against national benchmarks.

Growing interest in data use is reflected by the growing body of literature on this topic. Recently, several special journal issues and edited volumes have been dedicated to this topic (Coburn \& Turner, 2012; Schildkamp, Ehren, \& Lai, 2012; Schildkamp, Lai, \& Earl, 2013; Turner \& Coburn, 2012). Hamilton et al. (2009) make recommendations regarding the use of student achievement to support instructional decisions based on the available 
evidence regarding data use. However, they also conclude that few studies draw firm conclusions on the effects of data use, and that recommendations are based primarily on case studies, descriptive studies, and expert opinions (Hamilton et al., 2009). In her literature review of data use in education, Marsh (2012) recognized a similar trend: the majority of studies regarding data use are descriptive (e.g. case study design, interviews, focus groups, observations, document analysis). However, some studies show that the use of data to reflect on and to adapt education can improve student achievement (Campbell \& Levin, 2008; Carlson, Borman, \& Robinson, 2011; Faber \& Visscher, 2014; Lai \& McNaughton, 2013).

\section{Defining DBDM}

Ikemoto and Marsh (2007) use the following broad definition of DBDM: "teachers, principals, and administrators systematically collecting and analyzing data to guide a range of decisions to help improve the success of students and schools" (Ikemoto \& Marsh, 2007, p. 108). At the class, school and board levels, student and school performance data are supposed to be analysed, and decisions are supposed to be based on these data. Since the aim of DBDM is to systematically maximize student achievement of all students, the focus is explicitly on evaluating and analysing student performance data. Standardized test results are the starting point, but ideally additional information also is gathered because no single assessment can provide all the information necessary to make informed decisions (Hamilton et al., 2009). Based on standardized test results, teachers come to understand their students' strengths and weaknesses, and they can use curriculum-based tests, classwork, homework and classroom observations to help determine students' instructional needs.

In the DBDM literature, the term 'decisions' implies a variety of actions that can be undertaken on the basis of data, such as: setting goals, adapting instruction, adapting the curriculum, evaluating the effectiveness of programs and practices, improving policy and reallocating time and resources as necessary (Earl \& Katz, 2006; Hamilton et al., 2009; Ikemoto \& Marsh, 2007; Mandinach, Gummer, \& Muller, 2011). In the schematic overview of DBDM (Figure 1), 'decisions' are decomposed into 'setting goals' based on the data, and 'determining strategies' in order to achieve those goals.

The goals set and the strategies chosen for goal accomplishment vary according to the level of decision making. At the group level, teachers can use student performance data to differentiate instruction (Dunn, Airola, Lo, \& Garrison, 2013). Teachers first set goals in terms of desired achievement gains or skill attainment. To accomplish those goals, they can (for example) decide to use a specific instructional strategy, or form a separate group of students to work on improving a specific skill. At school and board levels, data is used to highlight specific areas for improvement in the school(s), and the strategies chosen often comprise policy decisions or (for example) the allocation of resources or the modification of the curriculum.

The final step is to implement and execute the chosen strategies. As Bennett (2011), Anderson, Leithwood, and Strauss (2010) and others have argued, the effects of these implementation activities are closely related to the quality of the inferences drawn on the basis of the data, the chosen approach to addressing the identified problems, and the instructional expertise of those working in schools. If, for example teachers draw incorrect conclusions about students' learning needs, they are likely to implement a strategy that is unlikely to lead to the desired outcomes.

Since DBDM is intended to be implemented as a systematic approach, data are also supposed to be used for monitoring and evaluating the effects and outcomes of the 
implemented strategies, for evaluating the extent to which goals have been achieved, and for making new data-informed decisions.

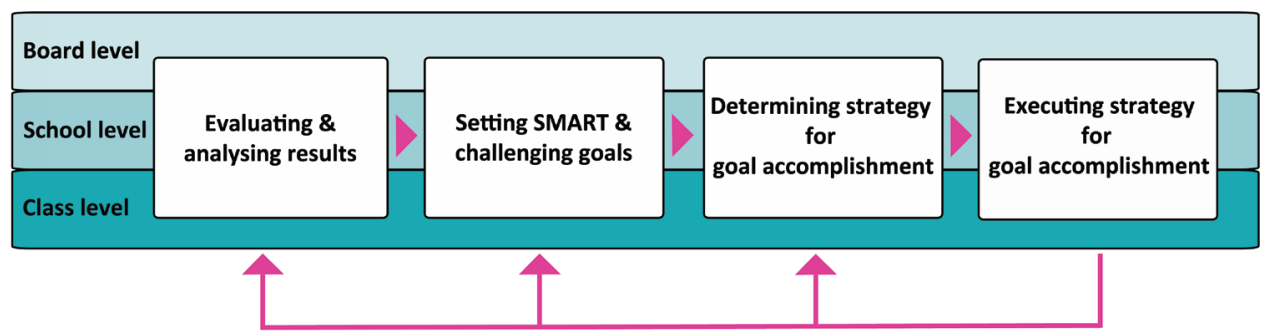

Figure 1. Schematic overview of DBDM

\section{DBDM - Why Should it Work?}

As mentioned earlier, there is little empirical evidence demonstrating the desired effects of data use as a 'package' of the four components as shown in Figure 1. However, research has demonstrated the effects of the separate components of DBDM. In this section, the scientific basis for each of those components of data-based decision making is discussed.

The first component in the DBDM model is the use of performance data to analyze and evaluate student results. This can be regarded as using performance feedback: student monitoring systems provide feedback to schools and teachers, in fact they reflect how students, teachers, and schools perform in comparison with the national average performance level, whether students' progress is adequate, and how students perform on subject matter content elements. The positive, performance improving effects of using feedback have been shown in several reviews and meta-analyses (Black \& Wiliam, 1998; Fuchs \& Fuchs, 1986; Hattie \& Timperley, 2007; Hattie, 2009; Kluger \& DeNisi, 1996) although recently the evidence has come to be questioned (Kingston \& Nash, 2011). Studies investigating the use of performance feedback in an educational setting (for example Coe, 2002; Gray, 2002; Oláh, Lawrence, \& Riggan, 2010; Vanhoof, Verhaeghe, Van Petegem, \& Valcke, 2013), identified critical features of effective student performance feedback in schools. Among other things, the degree to which the feedback recipients obtain an idea of how they can improve is very important. Information that one is underperforming without an idea of the cause of underperformance and about how performance may be improved makes demotivation more likely than improving performance. Furthermore, clear graphical representation of data is crucial for improving understanding and using the performance feedback (Verhaeghe, 2011).

The second element of DBDM - setting SMART and challenging goals - also has a proven effect on performance. Locke and Latham (2002), who developed their goal setting theory for various types of tasks, have shown that setting challenging and SMART (Specific, Measurable, Attainable, Relevant, Time-bound) learning, or performance goals significantly helps in improving performance. Goal setting in combination with the use of feedback improves performance even more (Locke \& Latham, 2002). The mechanism that explains such goal setting effects is that difficult but attainable goals in general motivate people. Explicit SMART goals also make people focus their activities more than otherwise (leading to less variation in the definition of goals, reducing activities that do not contribute to goal accomplishment, and increasing time on task), and promote the search for and utilization of task-relevant knowledge leading to improved performance (Morisano \& Locke, 2013).

The third and fourth elements of DBDM concern choosing and executing a strategy 
for goal accomplishment. Although the type of strategy chosen is dependent on the level of decision-making (group, school or board), in general all strategies are aimed at improving instruction, and consequently at improving student achievement. It is generally accepted that what teachers do in the classroom is the most important malleable factor influencing student performance. Although the impact of student characteristics is considered to be greater, such factors can only be influenced to a limited degree, and instruction is in any case essential for such influence (Hanushek, 2011; Hattie, 2009; Nye, Konstantopoulos, \& Hedges, 2004). Therefore, it makes sense to search for ways to improve the quality of teacher behavior, since ultimately it is the quality and execution of the chosen instructional strategies that are decisive for increasing student achievement.

Since DBDM includes several elements of which the effects were described in the previous paragraphs, the question remains whether the effect of the whole DBDM 'package' is greater than the sum of its parts. In the following section, the available scientific evidence for DBDM as a whole will be discussed.

\section{Research on Data Use}

In their introduction to their data use special issue, Turner and Coburn (2012) stated that all interventions to promote data use are "rooted in the conviction that if the right data are collected and analyzed, they will provide answers to key educational questions and inform actors' decisions, and better educational outcomes will follow" (Turner \& Coburn, 2012, p. 2). However, as described earlier, few causal studies on the effects of data use are available, and the existing literature does not always provide us with the answer to the question when and under what conditions data use interventions lead to the ultimate outcomes: improved student achievement.

Four aspects of the available studies on data use are important in this context (Coburn \& Turner, 2012; Hamilton et al., 2009). First, studies are often descriptive: the nature and background of interventions are described, but the implementation or effects are not analyzed. For example, Wayman and Cho (2008) advocated that teachers should be prepared to use data systems and also which approach could be suitable, and what preconditions should be met, but they did not go beyond making recommendations. In other studies, only practical aspects regarding the implementation of DBDM are described. Supovitz (2012), for example, investigated how tests can be designed that can maximize feedback to teachers, and Wayman, Stringfield and Yakimowski (2004) and Vanhoof et al. (2013) focused on technical and graphical aspects of performance representations in school performance feedback systems.

Second, the outcomes or the process of data use interventions are examined, but there is no published research that had looked into both of these effects together. Based on Desimone (2009), one may argue that the introduction of DBDM will lead to increased teacher quality, which will lead to improved teaching, and that this will finally lead to better student results. Information on the changes and effects on all subsequent stages in this process is necessary in order to be able to design effective interventions that lead to desired outcomes via effective processes.

Third, the focus of studies is often on aspects of the organizational context, such as leadership, school characteristics, policy environment and political context, although little is known about the interactions between all those contextual factors.

Fourth and finally, in many studies causal claims are made without a research design that justifies such claims: data use was often not examined in real school contexts 
and scholars relied mainly on self-reports and retrospective forms of data collection (Turner \& Coburn, 2012).

Based on insights from the literature on data use, professional development and comprehensive school reform, a DBDM intervention was designed. After a pilot study and the first project ran in 43 schools in the Netherlands (Staman, Visscher, \& Luyten, 2013, 2014), the intervention was optimized on the basis of experiences and new insights, and was implemented as 'the Focus intervention' in 53 schools during the school years 2011-2012 and 2012-2013.

\section{The Intervention}

The Focus intervention is a two year training course for entire primary school teams (all teachers as well as the members of the management team such as the school leader and deputy director), aimed at acquiring the knowledge and skills related to DBDM and implementing and sustaining DBDM in the school organization by means of the training activities as depicted in Figure 2. The training course and accompanying protocols and documents were developed by the University of Twente, but participating schools were stimulated to adapt these in order to fit their specific context. School leaders were supported in fulfilling the conditions in terms of school leadership, school culture, and professional networks and collaboration.

\begin{tabular}{|c|c|c|c|c|c|c|c|c|c|c|c|}
\hline Year 1 & 1 & 2 & 3 & 4 & $S$ & & 5 & & 6 & $S$ & 7 \\
\hline & $\mathrm{Se}$ & & Oct & Nov & Dec & Jan & Febr & Mar & Ap & May & June \\
\hline Year 2 & & 1 & 2 & & $S$ & & 3 & & 4 & S & 5 \\
\hline
\end{tabular}

Figure 2. Overview of the intervention. Numbers indicate team meetings, Sindicates meetings with trainer and school leader

First, practical preconditions needed to be fulfilled in order to make DBDM possible. Therefore, the availability of assessment tools (standardized tests) and technological tools (a student monitoring system) was a requirement for participation in the training. Furthermore, prior to the first meeting, a meeting with the school leader and school board was organized to stress the importance of their role in encouraging, motivating and supporting their team members. This meeting was also organized to assure that they would allocate sufficient time to their team members to work on DBDM activities such as analyzing data, planning and evaluating instruction, and that other practical preconditions (e.g. the availability of a SMS) were also fulfilled.

During the first year of the training, the subsequent steps of DBDM (Figure 1) were introduced one by one. The first four meetings were primarily dedicated to working on the knowledge and skills related to DBDM: using the student monitoring system, analyzing and interpreting test score data, diagnosing learning needs, setting goals and developing instructional plans. Next to the knowledge and skills at the user level, beliefs, attitudes and motivation also play a role when introducing and implementing DBDM. Also at the level of the organization these factors are important for success: a vision on what is considered important, performance norms, goals to be accomplished, a culture of collaboration, and a culture of trust. No meetings were especially dedicated to overcoming resistance and creating motivation, but attention was paid to explaining and stressing the (expected and experienced) benefits of DBDM according to the scientific literature, and based on the experience of participants in the pilot study and the first tranche of the intervention training 
course.

During meeting 1.5 in the first year of the intervention, the cycle of DBDM was fully completed for the first time when student achievement results were discussed in a team meeting. It was stressed by the trainers, school leaders and participants that data were supposed to be used for improvement, and not for judging colleagues. This was supposed to contribute to a culture of trust, and of collaboration, in which the school team as a whole felt the responsibility for their students' performance. Meeting 1.6 focused at collaboration among team members by observing each other's lessons, either to learn from the colleague they visited, or providing him/her with feedback on specific topics.

As of meeting 1.7 in the first intervention year, the meetings were aimed at internalizing, sustaining and broadening the scope of DBDM in the school, and supporting participants with carrying out their decisions in practice, for example, by coaching sessions (meeting 2.4 in the second intervention year) in which the trainers were observing teachers in their classrooms, and provided them with feedback. During team meetings, attention was paid to issues that were raised by the school and based on their requests for help.

Next to the team meetings, the trainers met with school leaders and school boards twice a year (indicated with ' $S$ ' in Figure 2) to discuss their role in the innovation process, the school's progress, and the goals to be set for the upcoming period. During these meetings, the importance of encouragement and support from school leaders and school boards was stressed.

All schools started with DBDM for mathematics. After the first intervention year, participating schools either chose to continue with DBDM for mathematics, or to broaden the scope of DBDM to spelling. Halfway the second intervention year, schools that chose to continue with DBDM for mathematics could again choose to continue with mathematics, or to broaden to spelling.

\section{The Link with the Literature on Professional Development}

The training activities were based on the literature on professional development. In the following paragraphs, those aspects are described.

Time. It takes time to learn and change. Desimone (2002) stated "it can take anywhere from 5 to 10 years for a school to completely reform" (p. 455). 'Duration' therefore is a structural feature of professional development in two ways: the number of contact hours and the time span over which the PD activity is spread (Birman, Desimone, Porter, \& Garet, 2000; Desimone, 2009; Garet, Porter, Desimone, Birman, \& Yoon, 2001). According to Timperley (2008), it takes typically one to two years for teachers to fully understand the promoted beliefs and practices, and to change practice. Due to many other obligations teachers face in their work, they should be provided with enough time to master the learning goals (Timperley, 2008; Van Veen, Zwart, \& Meirink, 2011). The time span of the intervention is two subsequent school years, a total of 22 months. Fourteen contact moments (each of approximately 4 hours) were planned, and in addition to these meetings, participants were expected to apply what they had learned in practice, for instance by carrying out analyses, developing instructional plans, and, finally adapting their instruction. Teachers gradually practiced and implemented what they had learned, which is also an important aspect of effective professional development activities (Timperley, 2008; Van Veen et al., 2011).

The entire school team has to participate. Collective participation (e.g. as a school team) is positively correlated with active participation in professional development activities. Garet et al. (2001), Lumpe (2007) and Van Veen, Zwart, and Meirink (2011) as well 
as Timperley (2008) argued that interaction with and collaboration between colleagues is important when implementing and mastering an innovation. In the Focus intervention, entire school teams participated in the intervention.

The use of protocols and documents. The implementation of comprehensive school reform is easier and faster in case of externally developed reform designs, often because they provide specific and detailed guidelines for implementation (Desimone, 2002). External experts who involve teachers in discussing and developing understanding, and who support teachers as they develop the understanding, the used tools (protocols and documents) are more effective (Timperley, 2008). In the Focus intervention, schools are therefore provided with protocols, documents and planning aids to help them incorporate DBDM in their organization and practice. During training sessions, these protocols and documents are discussed and adapted to the local school context because staff seems to support reform better when they are actively engaged in co-constructing the changes in their schools in such a way that the changes fit their local context (Datnow, Hubbard, \& Mehan, 1998). Datnow and Castellano (2000) describe the adaptations teachers made to the Success for All program, especially when they felt that their students' needs were not met by the prescribed program. Although these adaptations could affect program fidelity, some flexibility was needed to ensure continuing teacher support.

\section{A Hypothetical Model of DBDM and Student Achievement}

In Figure 3, the general model for this study is presented. It builds on previous studies on databased decision making which found that the use of data can improve student achievement (Campbell \& Levin, 2008; Carlson et al., 2011; Lai \& McNaughton, 2013). In this multilevel model, it is hypothesized that implementing DBDM will lead to (unmeasured) changes in a teacher's classroom practices which, in turn, will lead to student achievement growth in mathematics (hypothesis 1), and furthermore that intervention effects will differ between schools (hypothesis 2).

At the school level, the effect of the implementation of DBDM are expected to vary as a result of school characteristics such as school size, average student SES, and the level of urbanization. Schools with a higher percentage of students with a lower socio-economic background on average score less well than schools with a high-SES student population (Carlson et al., 2011; Inspectie van het Onderwijs, 2012). Since teachers are more likely to underestimate the potential of students from a low-SES background, an interaction between intervention and average school student-SES is expected (hypothesis 3 ) because the intervention is aimed at ambitious goal setting by teachers, and improving the educational achievement of all students.

At the student level, achievement might differ based on students' gender, SES, initial achievement, and the grade they are in at the moment of testing: therefore achievement will be controlled for these background characteristics. At the student level, comparable with hypothesis 3 at the school level an interaction effect is expected for SES and the intervention: the intervention effect is expected to be higher for low-SES students (hypothesis 4).

Furthermore, as mentioned in the previous, schools chose one out of three intervention trajectories at the end of the first intervention year. It was expected that schools in which DBDM for mathematics was implemented successfully during this first intervention year, chose to continue with DBDM for spelling immediately at the start of or halfway through the second intervention year. Therefore, the intervention effect will probably be greatest for schools following the mathematics-spelling-spelling variant, smaller for the mathematics- 
mathematics-spelling trajectory, and smallest for schools that decided they needed the full two intervention years to implement DBDM for mathematics (hypotheses $5 a$ and $5 b$ ).

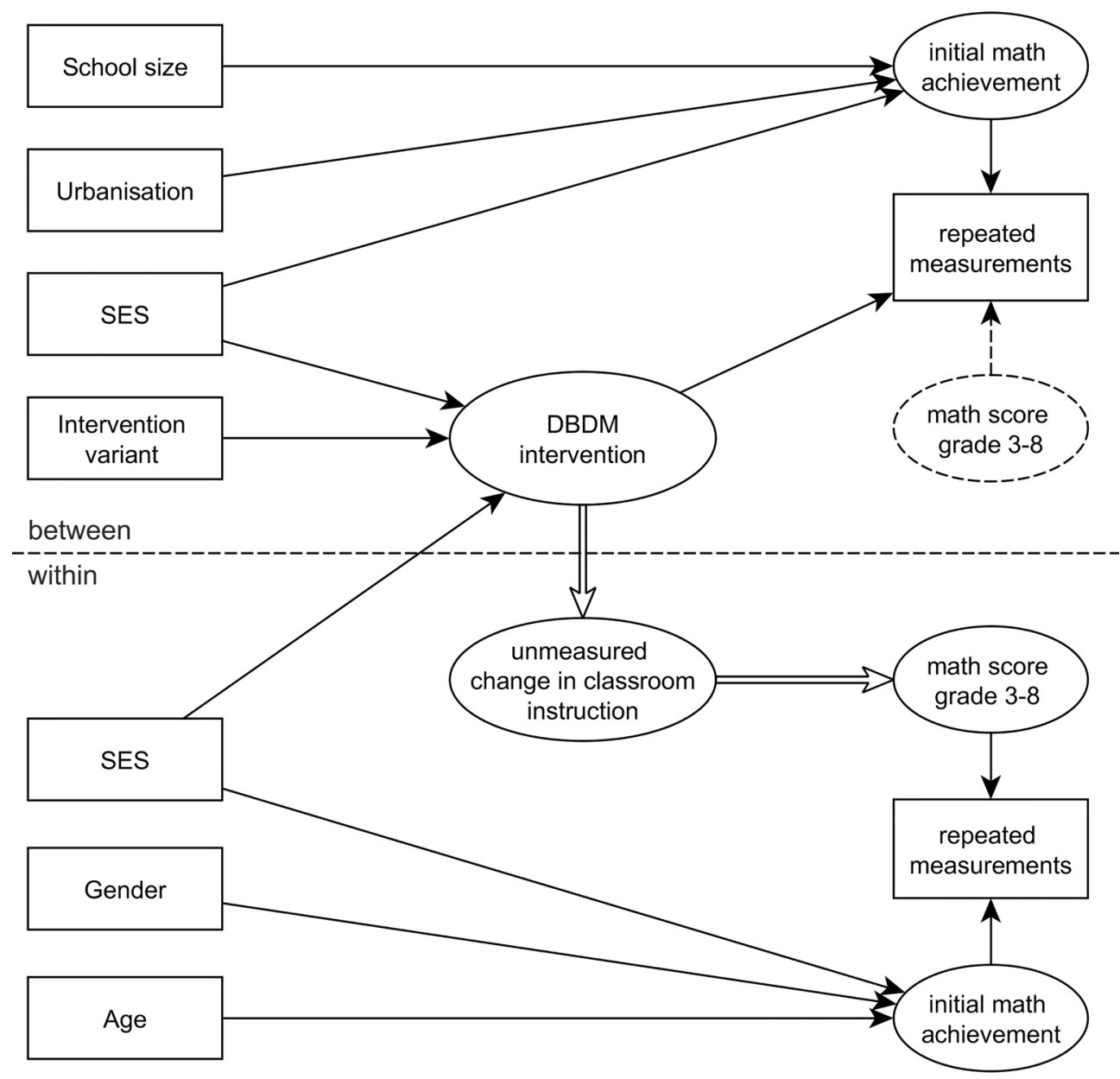

Figure 3. Conceptual model of the relationship between DBDM and student achievement growth

Following the conceptual model, as given in Figure 3, a multilevel growth model can be specified for the repeated measurements, which are nested in grades, students, and schools. Let $Y_{\text {tgij }}$ denote the performance measurement of student $i(i=1, \ldots, N)$ on test occasion $t(t=1,2)$ in grade year $g(g=3,4, \ldots, 8)$ in school $j(j=1, \ldots, J)$. The test occasion $t$ corresponds to the two test occasions in each grade year (mid, end), from the third to the eighth grade. The time between measurement occasions (i.e., the follow-up times) were more or less uniform across students and schools. A multilevel modeling approach is used to handle differences in the number of measurements per grade year, per student, and the number of students per school.

It is assumed that a student's scores in each grade year are independently normally distributed given the population-average occasion-specific score and the grade-year average 
student's performance, representing the student's deviation from the population average in grade $g$. Then, the level-1 part of the multilevel model for the grade- $g$ measurements can be represented as

$$
y_{t g i j}=\mu_{t g}+\delta_{g i j}+e_{t g i j}
$$

where $\mu_{t g}$ is the population-average performance on test $t$ in grade year $g$ and $\delta_{g i j}$ the individual variation in the performance of student $i$ in school $j$ in grade year $g$. The error component, $e_{t g i j}$, is assumed to be independently and normally distributed. The $g=6$ individual random components, $\delta_{i j}=\left(\delta_{3 i j}, \ldots, \delta_{8 i j}\right)$, representing the student's deviation from the population-average performance in each grade, are assumed to be multivariate normally distributed with mean zero and a common covariance matrix across students. These student's performance deviations are assumed to be correlated over grades, to model the covariance structure of each student's longitudinal measurements given the occasionspecific population performances.

The student-level modeling part is a function of the school-average performance (i.e., deviation from the population average) and a random error term. The school-average performance consists of a random component and an additional random intervention effect, which represents the school-average change in performance due to the intervention. The random effect of this intervention is assumed to be a school-specific effect but homogenous over grades and over the intervention period. Let the intervention variable, denoted as Int ${ }_{g i j}$ for student $i$ in school $j$ and grade $g$, equal one, when the measurement was observed during the intervention, and equal zero otherwise. Then, the grade- $g$ performance of student $i$ in school $j$ is represented by

$$
\delta_{g i j}=\beta_{0 j}+\beta_{1 j} \operatorname{Int}_{g i j}+u_{g i j}
$$

where $\beta_{0 j}$ is the mean achievement of school $j$ representing the deviation of school $j$ from the population average before the intervention, and $\beta_{1 j}$ the additional contribution in performance of the school during the intervention period. Furthermore, $u_{g i j}$ is the random variation in students's performances from the school-average performance in grade $g$ and school $j$, which is assumed to be independently and normally distributed.

Finally, the school-level part of the model is represented by,

$$
\begin{aligned}
& \beta_{0 j}=\gamma_{00}+r_{0 j} \\
& \beta_{1 j}=\gamma_{10}+r_{1 j}
\end{aligned}
$$

where $\gamma_{00}$ is the average performance in the population but equals zero, when $\mu_{t g}$ defines the population average scores. The component $r_{0 j}$ represents the random deviation of school $j$ in average performance. The $\gamma_{10}$ is the population-average change in performance during the intervention and $r_{1 j}$ the random deviation of school $j$ in the change in average performance during the intervention. The error components $\left(r_{0 j}, r_{1 j}\right)$ are assumed to be multivariate normally distributed.

The explanatory variables, as represented in Figure 3, can be incorporated in the multilevel model for repeated measurements to explain variation in the random student, school and intervention effects. 
Each school is repeatedly measured over time before the intervention period (the control phase) and during the intervention period (the treatment phase). The purpose is to measure the change in scores (i.e., performance of each school) and to assess the impact of the intervention for each school. Jenson, Clarck, Kircher and Kristjansson (2007) and Van den Noortgate and Onghena (2003) advocated the use of hierarchical linear models to improve the statistical inferences. The present design research extends the hierarchical linear model modeling approach of single-subject design studies, by extending the level-1 model for the repeated measurements of a single-subject study. Through the joint modeling of multiple single-subject designs, each single-subject study of a school concerns multivariate repeated measurements of students (representing the school), who are followed over time.

The proposed multilevel modeling approach overcomes the common problems to make accurate statistical inferences from a single-subject design study. The typical serial correlation between single-subject observations are modeled with random student effects such that the correlation will not bias the residual errors, parameter estimates and standard errors. In contrast to the typical small sample sizes, which are used in single-subject studies, much more reliable and accurate school-specific intervention effects can be obtained by pooling the information from all schools, and by combining the results from multiple single-subject design studies (e.g. Gage \& Lewis, 2014). The results can also be more easily generalized through the proposed multilevel modeling approach.

\section{Methodology}

The data used in this study were collected before, during and after the implementation of DBDM by means of the Focus intervention in 53 Dutch primary schools. In this section, first the participants, measures, and data collection are described, after which the section ends with a description of how the data were analyzed.

\section{Participants}

In November 2010, over 500 primary schools in the northern and central parts of The Netherlands were invited to attend a project briefing in their region in order to determine whether they would like to participate in the project. In total, eleven project briefings were organized which led to 55 participating schools. Two schools chose not to continue with the intervention after completing the first year: their school leaders argued that their teams already implemented the DBDM way of working for other subject areas and therefore did not see added value in participating for another year.

In total, 53 schools (1190 team members) fully participated in the study. Their characteristics are presented in Table 1. School teams included on average 22 team members, with a range from 5 to 67. School size was on average 245 students (range 55-806), and was categorized into small, medium and large. School-SES (categorized in high, medium and low) was based on the percentage of students who had been assigned extra 'weight' based on parental educational level indicating low SES'. Approximately half of the schools (23) were suburban schools, eleven were situated in big cities (urban), and 19 schools were located in rural areas.

Regarding the intervention trajectory variants: 15 schools chose to work on DBDM

1 Students are assigned extra 'weight' if their parents are from a lower educational background. Students can get an extra weight of 0.3 (maximum parental educational level of lower vocational education), or 1.2 (maximum parental educational level of primary education, or special needs education). Schools receive additional funding based on student weights as it is assumed that schools with students with student weight have a more difficult job to do. 
for mathematics in both intervention years (version MMM), 25 schools started with DBDM for spelling at the beginning of the second year (MSS), and 13 schools started with spelling halfway the second intervention year (MMS).

Table 1. Sample characteristics of schools $(N=53)$

\begin{tabular}{llcc}
\hline & & N & (\%) \\
\hline School size & Small $(<150)$ & 14 & $(26 \%)$ \\
& Medium (150-350) & 31 & $(58 \%)$ \\
School-SES & Large $(>350)$ & 8 & $(15 \%)$ \\
& High & & \\
& Medium & 17 & $(32 \%)$ \\
& Low & 24 & $(45 \%)$ \\
Urbanization & Rural & 12 & $(23 \%)$ \\
& Suburban & & \\
& Urban & 19 & $(36 \%)$ \\
& & 23 & $(43 \%)$ \\
Trajectory & M-M-M & 11 & $(21 \%)$ \\
& M-M-S & & \\
& M-S-S & 15 & $(28 \%)$ \\
& & 13 & $(25 \%)$ \\
& & 25 & $(47 \%)$ \\
\hline
\end{tabular}

\section{Measures and Data Collection}

The intervention took place from August 2011 until July 2013. In order to compare achievement growth during the intervention with achievement growth before implementing DBDM, student achievement data were collected from August 2009 until July 2013. The data were retrieved from the student monitoring systems of the schools participating in the intervention.

The student achievement on standardized tests were scored on an ongoing ability scale per subject, from grade three to eight (students aged six to twelve years old, all primary school grades). Students take these tests twice a school year (mid and end of school year) with an exception for grade eight, where the test at the end of the school year is scaled differently. The test at the mid-occasion however, can also be taken at the beginning of the school year, but students can only take this test once. This means that there are eleven standardized assessments per student per subject over the course of their primary school career. Over the two years before the intervention and the two intervention years, most students took eight tests, leading to eight ability scores per subject, which makes it possible to follow student cohorts and to compare achievement of grades across years. An overview of test occasions is depicted in Figure 4. With approximately 1,500 observations per grade per test moment per school year, the total of observed achievement scores was 66,486.

Next to students' ability scores, the following data was collected at the student level: gender, student weight category indicating SES, and date of birth. Age was centered based on the expected age in months at the time of the test, based on the average age for students who do not accelerate or repeat grades, and thus indicating how many months younger or older a student was than expected.

At the school level, data was collected on school size, degree of urbanization, average SES, and intervention trajectory variant. 


\begin{tabular}{|c|c|c|c|c|c|c|c|c|}
\hline & \multicolumn{4}{|c|}{ Prior to intervention } & \multicolumn{4}{|c|}{ During intervention } \\
\hline & \multicolumn{2}{|c|}{ School Year 2009-2010 } & \multicolumn{2}{|c|}{ School Year 2010-2011 } & \multicolumn{2}{|c|}{ School Year 2011-2012 } & \multicolumn{2}{|c|}{ School Year 2012-2013 } \\
\hline & $\begin{array}{l}\text { Mid } \\
\text { (Febr) }\end{array}$ & $\begin{array}{c}\text { End } \\
\text { (June) }\end{array}$ & $\begin{array}{l}\text { Mid } \\
\text { (Febr) }\end{array}$ & $\begin{array}{l}\text { End } \\
\text { (June) }\end{array}$ & $\begin{array}{l}\text { Mid } \\
\text { (Febr) }\end{array}$ & $\begin{array}{l}\text { End } \\
\text { (June) }\end{array}$ & $\begin{array}{l}\text { Mid } \\
\text { (Febr) }\end{array}$ & $\begin{array}{l}\text { End } \\
\text { (June) }\end{array}$ \\
\hline Grade 3 & $x$ & $x$ & $x$ & $x$ & $x$ & $x$ & $x$ & x \\
\hline Grade 4 & $x$ & $x$ & $x$ & $x$ & $x$ & $x$ & $x$ & $x$ \\
\hline Grade 5 & $x$ & $x$ & $x$ & $x$ & $x$ & $x$ & $x$ & $x$ \\
\hline Grade 6 & x & $x$ & $x$ & $x$ & $x$ & $x$ & $x$ & $x$ \\
\hline Grade 7 & $x$ & $x$ & x & x & $x$ & $x$ & $x$ & $x$ \\
\hline Grade 8 & $x$ & & $x$ & & $x$ & & $x$ & \\
\hline
\end{tabular}

Figure 4. Overview of measurement occasions (shadings indicate cohorts)

\section{Data Analysis}

Given the multilevel structure of the data, with measurements nested within students, and students nested within schools, the Ime4 package (Bates, Mächler, Bolker, \& Walker, 2014) in R (RCoreTeam, 2013) was used to perform linear mixed effects analyses to investigate and assess effects of the intervention on student achievement.

For each student, an incomplete set of measurements was observed. In the four years of the study, a maximum of eight measurements was observed of the in total eleven measurements (from grade three to grade eight, see also Figure 4). Therefore, it was not possible to estimate a random performance effect for each student in each grade, and to model the change in performance from grade three to grade eight for each student. The in total six individual random effects, referred to as $\delta_{g i j}(g=3, \ldots, 8)$, was reduced to three, based on the common combination of grades into middle and higher grades units. Each student's changes in performance over grades was modeled using a random initial level (first test occasion in $3^{\text {th }}$ grade, referred to as $\left.\tilde{g}=1\right)$, a middle $\left(3^{\text {th }}-5^{\text {th }}\right.$ grade) grades level $(\tilde{g}=2)$, and a higher $\left(6^{\text {th }}-8^{\text {th }}\right.$ grade) grades level $(\tilde{g}=3)$. This reduced level-1 random effects structure corresponds in a more efficient way to the incomplete data structure and also avoids the missing data problem in estimating all random effects. The individual random effects $\delta_{\tilde{g} i j}(\tilde{g}=1,2,3)$ were assumed to be multivariate normally distributed. A positive covariance between individual random effects was expected, which defined the correlation between each student's latent performances.

The differences in population-average achievements over measurement occasions were modeled as fixed effects such that the general mean represents the average performance of students over schools at measurement occasion mid-year grade three. Student and school achievements were allowed to vary across the general mean, which was accomplished by the individual-specific and school-specific random effects. At the level of schools, a random effect was introduced to model the average differences in achievements between schools over grades.

The three individual random effects were used to model each student's (average) deviation from the population-average scores in mid-grade three, grade years three to five, and grade years six to eight. This led to three separate latent measurements over time, representing growth in student performance given the population average scores. The individual random effects capture the heterogeneity in average achievements in the lower and upper grades over students given differences in population-average achievement over students and schools between test occasions.

A second school random effect was introduced to model the difference in average performance of schools before the intervention and during the intervention. By modeling 
the differential effect of this intervention effect, school-specific intervention effects were estimated and schools benefiting from the intervention were identified. This mixed effects model for the individual scores is given by,

$$
\begin{aligned}
& Y_{t g i j}=\mu_{t g}+\delta_{\tilde{g} i j}+e_{t g i j} \\
& \delta_{\tilde{g} i j}=\beta_{0 j}+\beta_{1 j} I_{n t_{g i j}}+u_{\tilde{g} i j} \\
& \beta_{0 j}=r_{0 j} \\
& \beta_{1 j}=\gamma_{10}+r_{1 j},
\end{aligned}
$$

which corresponds to the model in Equation 1 to 3, except for the reduction in level-1 random effects.

To test the specific hypotheses and to explore the effects of the intervention, several multilevel models were fitted. In the null models (Model $\mathrm{Oa}$ and $\mathrm{Ob}$ ), student and school achievements were modeled through random effects, while accounting for differences in average achievements over assessments. Subsequently, heterogeneity in intervention effects among schools were estimated, given the growth specification of student achievements. In the subsequent models, the average intervention effect (Model 1), student background characteristics (Model 2), school characteristics (Model 3), intervention trajectory (Model 4), and interaction effects (Model 5) were added. Non-significant effects were not included in the next model. A detailed explanation of the equations for each model is provided in Appendix A.

\section{Results}

Basic descriptives of ability scores, per grade and by intervention status, are presented in Table 2. The results of the analyses of the relationship between student achievement and the implementation of a DBDM intervention are presented in Table 3. Based on decrease in information criteria values (i.e., AIC, BIC, Deviance), each subsequent model was a significant $(p<.001)$ improvement compared to the previous one, except for Model 4 as compared to Model 3.

Student achievement was measured using standardized tests with a national benchmark. Based on the benchmark data, the estimated average difference between student scores at two subsequent test moments is approximately 7.7 (Cito, 2009a). From Table 2 and 3 it follows that differences in average scores of the same magnitude were found between subsequent assessments. Since there are approximately five school months between two test occasions, an effect of 1.54 (average of 7.7 ability points, divided by five months of schooling) on average can be interpreted as the expected increase in performance due to one additional month of schooling. This expected effect of an additional month of schooling will differ slightly between lower and higher grades, since the estimated differences in ability scores between two test occasions are larger in the lower grades (Cito, 2009a). 
Table 2. Mean ability score per grade, by intervention status

\begin{tabular}{lcccc}
\hline & \multicolumn{2}{c}{ Prior to intervention } & \multicolumn{2}{c}{ During intervention } \\
Mean (SD) & N & Mean (SD) & N \\
\hline Mid Grade 3 & $29.70(16.05)$ & 3077 & $31.25(15.93)$ & 3178 \\
End Grade 3 & $40.39(16.51)$ & 3066 & $43.07(16.21)$ & 3104 \\
Mid Grade 4 & $49.40(15.99)$ & 3100 & $51.07(15.55)$ & 3232 \\
End Grade 4 & $60.29(15.37)$ & 3141 & $62.35(15.57)$ & 3097 \\
Mid Grade 5 & $70.11(15.84)$ & 3146 & $71.48(15.08)$ & 3236 \\
End Grade 5 & $78.01(14.70)$ & 3169 & $79.14(14.51)$ & 3237 \\
Mid Grade 6 & $84.77(13.77)$ & 3046 & $85.52(13.01)$ & 3123 \\
End Grade 6 & $90.88(12.37)$ & 3043 & $91.29(11.67)$ & 3041 \\
Mid Grade 7 & $98.73(12.43)$ & 2775 & $100.16(12.20)$ & 3214 \\
End Grade 7 & $104.99(13.46)$ & 2679 & $105.88(12.81)$ & 3069 \\
Mid Grade 8 & $110.78(12.40)$ & 1453 & $112.72(11.64)$ & 2275 \\
\hline
\end{tabular}

\section{Baseline Model}

The achievement scores are measured on one common ability scale and students are expected to grow in ability between every two assessments. This average growth in achievements over test occasions is represented by the growth in average scores over students from grade 3 to grade 8 . The random intercepts show a significant variability in achievements over students at the first assessment. There is less variability between students in average achievements in grades three to five and grades six to eight when comparing them to the variability in achievements at the first assessment. It follows that the variability in achievements over students diminishes when students receive education over a longer time period. This corresponds to the fact that student achievements from the same school are more alike than those from different schools.

\section{Intervention Effects}

In Model Ob, the random intervention effect at the school level was introduced, leading to a significant decrease in deviance $\left(\Delta \chi^{2}=864.16,2 \mathrm{df}, \mathrm{p}<.001\right)$. It can be concluded that the intervention effect varied significantly across schools. These findings support hypothesis 2 (the intervention effect will differ between schools).

By modeling achievement differences between schools through a random intervention effect, the variance of the student random effects only slightly decreased. In Model Ob and Model 1, the random intercept at the student level represents differences in mid-grade three student achievements, while accounting for differences between schoolaverage performances prior to the intervention and during the intervention. Accounting for differences in school performances during and prior to the intervention did not influence the variability in student performances. However, in comparison to Model Oa, the school-level random intercept variance increased in Model $0 \mathrm{~b}$. This occurred due to the fact that the random intercept variance represents the variability in average mid-grade three achievements across schools prior to the intervention, and not over all measurement occasions. In Model $\mathrm{Oa}$, this random intercept variance represents the school-average achievements over time, where in Model $\mathrm{Ob}$ a distinction is made between the school-average achievements prior and during the intervention.

From Model 1 it can be concluded that the general average intervention effect differed significantly from zero, and equals 1.40. The random intervention effect is assumed to be normally distributed in the population of schools. Given the estimated random effect 
variance of 4.55 , the $95 \%$ confidence interval of intervention effects in the population ranges from-7.52 to $10.32(1.40 \pm 1.96 * 4.55)$. As a result, it can be concluded that the effect of the intervention will not necessarily be positive for all participating schools.

The estimated intervention effects are possibly biased due to missing confounding (background) variables. Therefore, it is important to include student and school background variables, which are known to be related to student achievement and possibly also to the intervention. These background characteristics were added in Model 2 and Model 3. Below, the estimated intervention effects will be explored and more profound explanations are given to explain variability in intervention effects.

\section{Exploring Student Effects}

Four schools did not provide data on student date of birth, and therefore information on student age related to the average age in their grade was missing for these schools. These schools were excluded from the further analyses, leading to a total of 49 schools as from this model.

Student background characteristics were added in Model 2. As is known from previous research such as TIMSS (Mullis, Martin, Foy, \& Arora, 2012), girls on average score lower than boys for mathematics. In this study the fixed effect for gender was-3.49 points on the ability scale, which can be interpreted as a lag of 2.3 educational months.

Another important factor in explaining variability in student achievement is studentSES. The Dutch student-SES weights are based on parental education, and previous studies, for instance TIMSS (Mullis et al., 2012) show that there are strong positive relations between the level of parents' education and their children's educational attainment. These findings are partially confirmed in the present study: high-SES students on average 6.43 points higher than medium-SES students (Table 3). It is remarkable that low-SES students on average also score higher than medium-SES students, although this effect is not significant.

Student age, centered around the expected age (in months) at each measurement occasion, represented the difference between the actual age and the average student age. The significant effect of age indicates that students above expected age score.43 points higher on the ability scale per month. Note that age is a time-varying variable and its effect represents average latent growth in student achievement. This effect cannot be interpreted as an argument for repeating grades. Students who repeat grades are older and therefore according to this model will score higher than their non-repeated classmates, however, test performances of students who repeat grades can be lower than the performance of students of the same age not repeating the grade.

When considering the random effects at the student and the school level, the student predictors explained variability at both levels. The differences in average student achievement at the lower and higher grades were reduced significantly. Note that including student characteristics did not lead to a change in the estimated average intervention effect, indicating a constant, positive main effect for intervention on student achievement. That is, differences in the achievements of students in the study prior to intervention and during intervention were not attributable to differences in observed student background variables.

\section{School Characteristics}

In Model 3, the school level characteristics school size, urbanization and SES were added (all were categorical variables). No significant effects were found for school size or level of urbanization. The effect of school-SES however is significant. Recall that school-SES is 


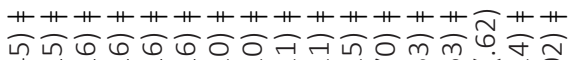

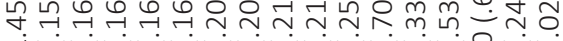
-

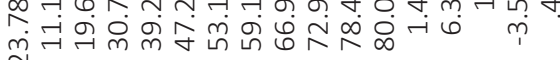

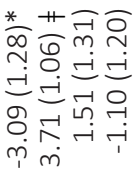

\#\#\#\#\#\#\#\#\#\#\#\#\#\#葓\#

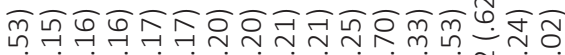
تं山N

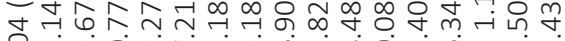

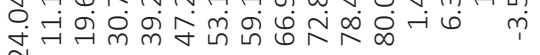

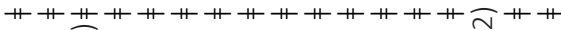

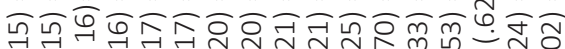

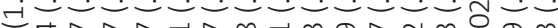
盯

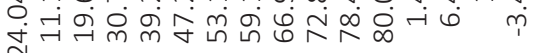

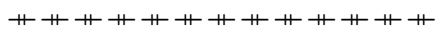

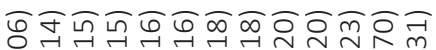
mㄷำ

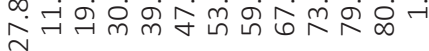

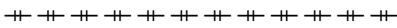
สิษ $=-1-10$ 는 लेન ન્ં

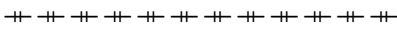

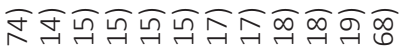

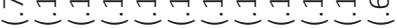

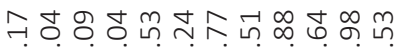

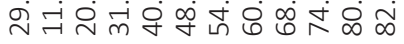

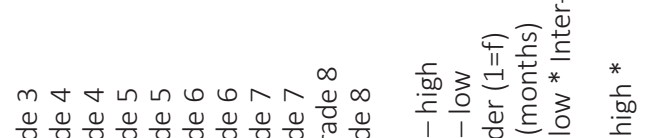
出 ब

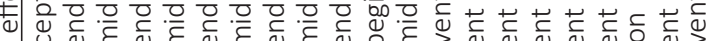

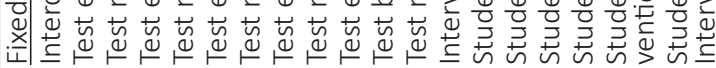

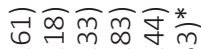

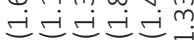

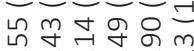
i 


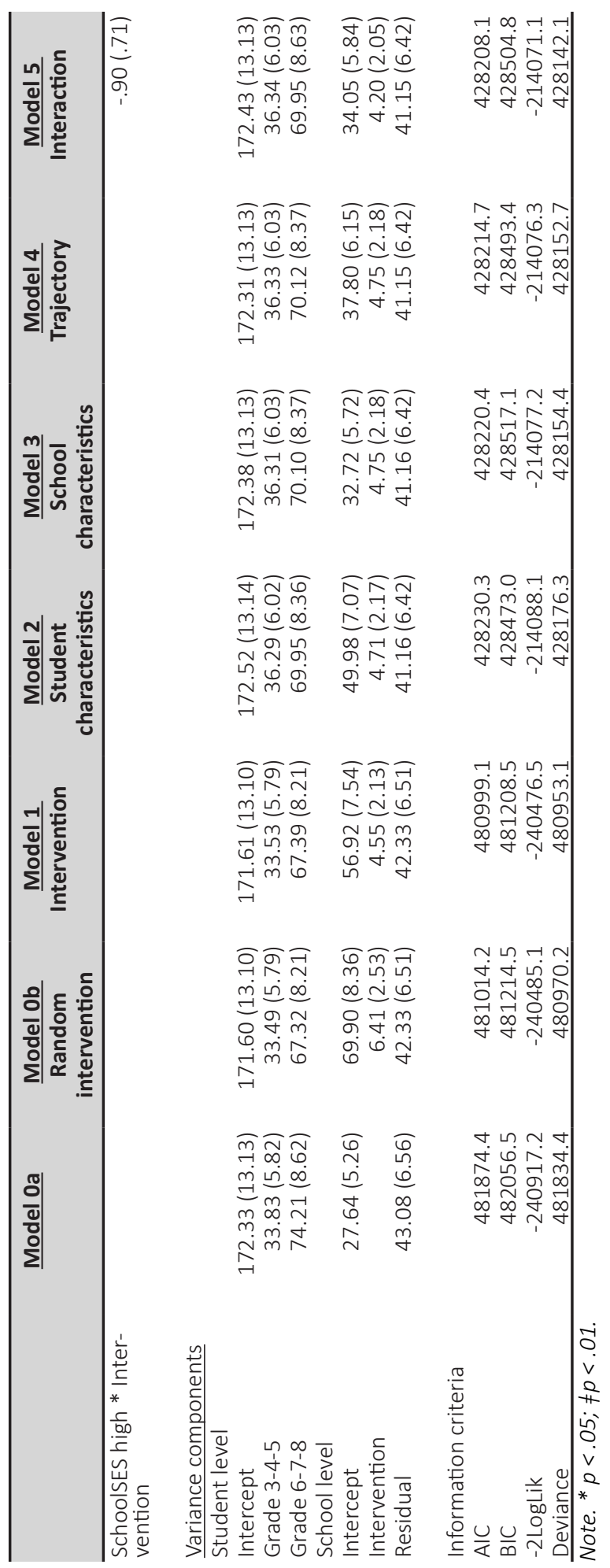


categorized into high, medium and low, and that medium is used as the reference category. Students in medium-SES schools score on average lower than schools with high-SES, and students in low-SES schools on average have lowest scores. All schools included in the analysis participated in the whole study. As expected, it followed that the observed background information of schools (in the period prior to intervention and in the intervention period) did not explain any variability in intervention effects.

According to Model 3, correlation between random intercept and random intervention effect was-.84 (see Table 3), indicating that the intervention effect is smaller for schools with high average achievement. The random intervention effect was plotted against random intercept in Figure 5, illustrating these findings. For illustrative purposes, shapes indicate school-SES. Surprisingly, schools with the highest intercept are not high-SES schools.

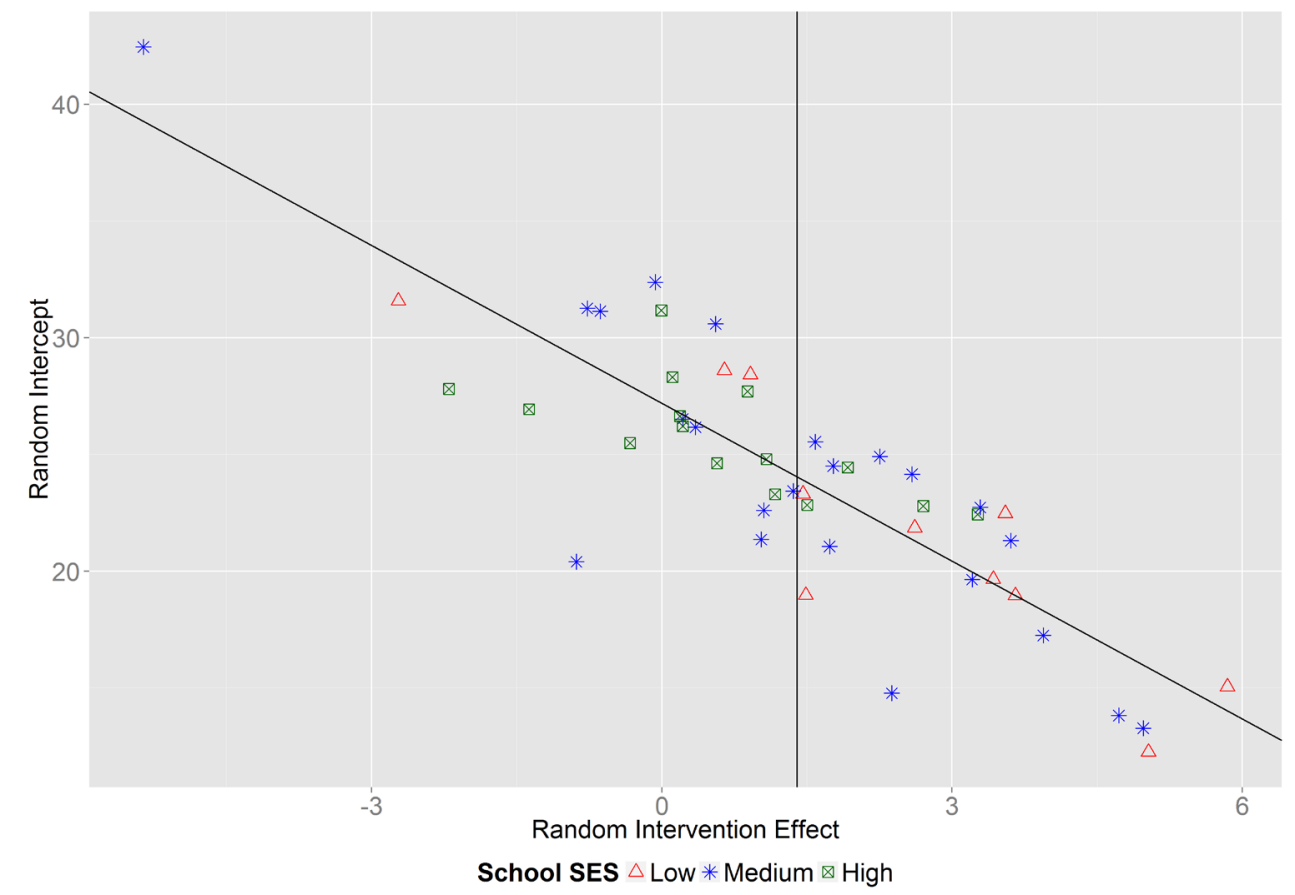

Figure 5. Random intervention effects plotted against random intercepts (Model 3)

\section{Intervention Trajectory}

The chosen intervention trajectory was not significant as a main fixed effect (Model 4). It was tested (not shown in Table 3) whether there was an interaction effect for trajectory and intervention, this was positive for the Math-Math-Spelling trajectory and negative for the Math-Spelling-Spelling trajectory, but these effects were far from significant. Hypotheses $5 \mathrm{a}$ (the intervention effect will be larger for schools that chose the M-S-S trajectory than for schools that chose the M-M-S trajectory) and $5 b$ (the intervention effect will be larger for schools that chose the M-M-S trajectory than for schools that chose the M-M-M trajectory) therefore have to be rejected. Student achievement did not differ significantly between schools choosing different trajectories, and no interaction effect with intervention was found. 


\section{Interaction Effects}

In Model 5, interaction effects were introduced for school-SES and the intervention, and for student-SES and the intervention. It was hypothesized that the intervention effect would be larger for schools with a large population of students with low-SES (hypothesis 3 ). The findings of this study support this hypothesis: the interaction effect for school-SES and intervention was positive but not significant for schools with low-SES, and negative but not significant for high-SES schools.

Hypothesis 4 concerned the interaction between intervention effect and studentSES. It was stated that the intervention effect would be larger for low-SES students. The interaction effect for intervention and low-SES student was significant and positive (effect of 1.05), but surprisingly the effect found for high-SES students and the intervention was also positive and significant (also an effect of 1.05).

Given that the interaction effect for student-SES and intervention is conditional for the interaction effect of school-SES and intervention, it is interesting to compare effects for the combination of student and school-SES even though the latter were not significant. These combinations indicate that the effect of the intervention will only lead to a negative effect on student achievement for medium-SES students in high-SES schools, and is positive and quite large for low- and high-SES students, regardless of their school's average SES.

It was assumed that school and student background characteristics would not influence the effect of intervention, and it is interesting to monitor the stability of the estimated intervention effect across models. The estimated positive main intervention effect and the random effect variability over schools is stable across Models 1 to 4 . Furthermore, the random intervention effect could not be attributed to differences in background information, or to differential growth in student and school achievement. Therefore, it is concluded that the random intervention effects are identified based on differences in achievement of students in the prior-to-intervention group and during-intervention group. The main intervention effect only decreased after including interaction effects in Model 5. These findings provide partial support for hypothesis 1 (the implementation of DBDM will lead to higher student achievement, there is a positive intervention effect).

Although the model fit results are not presented, the multilevel models were investigated with respect to model fit. For each model, a residual analysis was carried out to identify outliers and to investigate distributional assumptions of the residuals. The level-1 residuals showed nine outliers, which most likely stemmed from incorrectly entered observations in the student monitoring system. Some of the scores did not fall within the possible score range of the administered test. Some bias in the fitted values were detected because of observed scores of zero. Since the scores were assumed to be normally distributed and by ignoring this lower-bound, some negative fitted scores were obtained. This lowerbound problem was ignored since the few negative predicted scores did not influence the parameter estimates, which were based on a total of 66,486 observed scores.

The student random effects were approximately normally distributed, but the random student effect distribution for grade 6-8 scores was very peaked. The variability in random latent student scores in grade 6-8 was less than expected since the correlation between random effects (grade 3-5 and grade 6-8) was high. The variance in effects across students was relatively low, leading to a more peaked distribution of the random effect distribution of student scores in grade 6-8. However, this random effect was needed to properly represent the scores of students in grade 6 to 8 despite the high correlation with the scores grade 3 to 5 . 
The residual analyses at level 2 showed that large residuals were obtained for the school coded as 2310, where all students scored exceptionally high. The school could be marked as an outlier but there was no more information available. Finally, the assumption of homoscedasticity of level-1 variances was tested using the chi-square test (Snijders \& Bosker, 1999). It was found that the assumption of equal level-1 variances was rejected, where significance was easily obtained through the high number of students per school. A further investigation of the level-1 variances showed that for almost all schools the assumption of a common residual variance was acceptable. The extension to deal with heteroscedastic level-1 variances would complicate the model analysis significantly, and would only Improve the error distribution of a few schools.

\section{Conclusions and Discussion}

There is a worldwide interest in the use of data in order to help improve education. Many studies focus on the preconditions for successful data-based decision making, or describe the process of DBDM in schools, but only a very few empirical studies are available on the effects of DBDM on student achievement. The present study is intended to contribute to the international knowledge base on DBDM effects, by investigating heterogeneity in the effects of a DBDM intervention on student achievement for mathematics in 53 primary schools in the Netherlands.

The findings of this study indicate that DBDM can improve student achievement (hypothesis 1, confirmed), although effects differ across schools (hypothesis 2, confirmed). The fixed effect of intervention without introducing interaction effects is 1.40 , indicating an effect of almost an extra month of schooling during the two intervention years. Interaction effects suggest that DBDM is especially effective for schools with a large proportion of lowSES students (hypothesis 3, confirmed). Interestingly, the effects for interaction between student-SES and intervention were not completely in line with expectations (hypothesis 4, partially confirmed): the interaction effect was positive and significant for low-SES, but this was also the case for high-SES students. Combining the interaction effects of intervention and student-SES and school-SES leads to the conclusion that the effect of intervention will lead to a positive effect for bot low- and high-SES student, regardless of their school's SES, and will only lead to a negative effect on student achievement for medium-SES students in high-SES schools. An explanation might be that medium-SES students in high-SES schools often belong to the lower-scoring students. Since the intervention was aimed at raising achievement for all students, it is possible that teachers decreased the amount of time dedicated to the lowest scoring students in order to devote attention across all students more equally. However, this does not seem to hold for low-SES students. Further analysis of the data may provide more insight into this effect.

The present study investigated the effects of a DBDM intervention that was focused on all four components of data-based decision making, as shown in Figure 1: analyzing results; setting goals; determining a strategy for goal accomplishment; and executing the chosen strategy.

Based on the results of another, quite similar intervention project, it is known that this intervention can lead to a considerable improvement in the correct interpretation of student achievement data (Staman et al., 2014). However, especially teachers still proved to make some misinterpretations after the intervention. These misinterpretations can lead to less adequate goals and a less effective instruction strategy, resulting in lower student 
achievement growth than is possible.

Furthermore, the meta-analysis of the effects of the use of digital student monitoring systems (DSMSs) on student achievement by Faber and Visscher (2014) shows that the use of a DSMS was especially effective when it was implemented by small groups of teachers (up to 30 teachers), and when DSMS use was aimed at improving instruction for small groups of children. An explanation for this may be that the intervention intensity will be smaller when addressing all teachers in a school at the same time. Furthermore, adapting instruction for all students will be more difficult for teachers than adapting instruction to the needs of a selection of students (Faber \& Visscher, 2014). The small intervention effect found in the present study is in line with their research, since the use of data (e.g. by using the DSMS) was implemented schoolwide, and aimed at improving education for all students at the same time.

Moreover, according to this review, the effect of the use of digital student monitoring systems was greater when the systems provided teachers with suggestions for adapting their instruction (Faber \& Visscher, 2014). However, in the present study, the student monitoring systems used by the participating schools did not provide teachers with this kind of instructional suggestions. Based on anecdotal evidence from trainers in the project, the quality of analyses and instructional plans certainly increased during the intervention. The question remains however, of whether all teachers can master the professional skills needed to implement DBDM in daily practice and whether they are all able to adapt their instruction to the needs of all students in their classroom. From the reports of the Dutch Inspectorate of Education, it is known that half to two-thirds of the teachers in primary schools do not master complex skills as differentiation (Inspectie van het Onderwijs, 2013). Exploratory classroom observations by the trainers, during the intervention in this study, confirmed those findings and suggest that the execution of instructional plans can still be significantly improved. Due to the large number of participating teachers in this project, it was not possible to explicitly include the coaching of teachers in their classrooms.

The Netherlands' government promotes the use of data to improve education, but policy makers must be aware of the preconditions for implementing DBDM in practice. Acquiring skills related to the analysis of data, setting goals and developing plans such as in [the intervention], combined with coaching and support in the classroom is costly, but is expected to lead to larger intervention effects than found in the present study (Faber \& Visscher, 2014). For successful large-scale implementation, the combination of DBDM with classroom support or coaching is therefore recommended.

Previous research calls for more empirical studies in real school contexts (Turner \& Coburn, 2012). It proved practically to be infeasible to find schools who were willing to participate in an experimental setting for two subsequent school years, risking the chance of being assigned to a control group. In this study, the effect of implementing DBDM was therefore compared to student achievement in the same schools during school years before the intervention.

\section{Design Limitations: External and Internal Validity}

The study design can be recognized as a single-subject design. Each school is repeatedly measured over time before the intervention period (the control phase) and during the intervention period (the treatment phase). The object was to measure the change in scores (i.e., performance of each school) and to assess the impact of the intervention for each school. In the single-subject design, multiple schools can be measured repeatedly but 
interest is focused on the intervention effect for each school and not for a (sub-)population of schools. This typical advantage of the single-subject design was used to measure schoolspecific intervention effects. Therefore, the fact that schools applied to participate in the study and that schools were not randomly assigned (to a control or treatment group) did not influence the validity of the school-specific measurement of the intervention. The intra-school measurements showed that the intervention effect was real and the method was reliable also through the use of standardized tests and the common scale analysis over grades.

Furthermore, several measures were taken to meet criteria of internal validity. Repeated measurements were taken at the pre-intervention period to take control over different threats to internal validity. The repeated measurements in the pre-intervention period did not show clear patterns illustrative for threats as testing, maturation, instrumentation, and statistical regression (Kratochwill et al., 2010). Furthermore, the repeated measurements for each school were not single-subject observations but were aggregate measurements constructed from multiple student scores. Therefore, it was not likely that extreme (low or high) school-performances were measured due to for example sampling error or measurement error, which could highly influence the estimated intervention effect.

The student population for each school changed over time such that school measures were not based on a fixed student population. This diminished the possibility that some other event influenced the results. Schools in the study did not report any event which could influence a substantial amount of student performances to influence the estimate of the intervention effect. Furthermore, the average (between-school) intervention effect was based on the multiple within-school intervention effects, which can be considered to be robust to bias from event effects (e.g. Ferron, Moeyaert, Van den Noortgate, \& Beretvas, 2014).

Finally, there was a threat of selection bias due to the self-selection of schools to participate in a specific trajectory. Systematic differences between schools before the study could possibly relate to the different trajectories within the intervention. During the intervention period schools were not allocated to intervention trajectories at random, but were allowed to choose the trajectory of their preference after the first intervention year. The choice to continue with DBDM for mathematics, or broadening the scope of DBDM to spelling during the second intervention year was allowed to be made by schools in order to increase motivation and commitment. It was expected that this choice would be related to achievement gain during the first intervention year. However, analyses showed that there were no significant differences in achievement or intervention effects across trajectories (hypotheses $5 \mathrm{a}$ and $5 \mathrm{~b}$, both rejected). Therefore, it may be assumed that schools did not base their choice of an intervention trajectory on the student achievement results during the first intervention year. Furthermore, it was unlikely that the self-selection of schools to participate in the study influenced the results. Besides the intervention trajectories, there were no different intervention conditions used.

In contrast to this design, in a completely randomized design, schools would be assigned to a control or a treatment group. This would provide insight in the effect of the intervention on the group of schools but ignore each school's experience with the intervention. Although this would support measuring the general intervention effect for the population of schools, the school's population average intervention effect might not be that interesting, since it is to be expected that the intervention effect will differ substantially over schools. The average intervention effect will simply not apply to most of the schools, since schools show different changes in performances due to the intervention. Furthermore, it 
is not realistic to assume that schools can be assigned to a control phase for several years, which forbids them to participate in any other program to improve their performances.

However, the strength of the single-subject design is also its main limitation, since results cannot be easily generalized beyond the schools which were included in the study. From this perspective, the multilevel modeling of the multiple single-subject studies (i.e., multiple schools were followed over time) can be seen as the joint modeling of all these studies to generalize the results. In our approach, by introducing a random intervention effect, the outcomes of the single-subject studies can be combined. This leads to an estimate of the average intervention effect and of the variation in the effects across the schools in the study. In fact, the joint multilevel modeling approach overcomes typical issues associated with the single-subject design in providing scientific evidence (Gage \& Lewis, 2012; Jenson et al., 2007).

The schools in the study were self-selected and not sampled from a population of schools, and they might not be representative of all primary schools in the Netherlands. Compared to the total number of schools, schools from big cities were disproportionally represented, and participating schools had a higher than average proportion of students from a lower-SES background. Since the effect of intervention was greater for low-SES schools, the effect might be smaller for a sample containing more high-SES schools. In future work, student achievement data of a national representative sample will be collected to compare student achievement growth of schools participating in this intervention with actual average growth of all students in the Netherlands.

The support from the project team finished after the two intervention years, and therefore the continuing implementation and sustainability were schools' own responsibility. Since full implementation of schoolwide reform can take up to five years (Desimone, 2002), it will be interesting to monitor student achievement and DBDM implementation in the schools that participated in the intervention. Student achievement data in the first school year after completing the intervention will be collected in the summer of 2014 in order to estimate retention effects, and school leaders will be interviewed about the sustainability of DBDM in their school organizations.

Further research within this project will focus on the relationship between DBDM effectiveness and the preconditions for successful DBDM, such as school leadership, an achievement oriented culture, and collaboration within the school team. A follow-up project includes the coaching of teachers on the effective use of DBDM in their classrooms. 


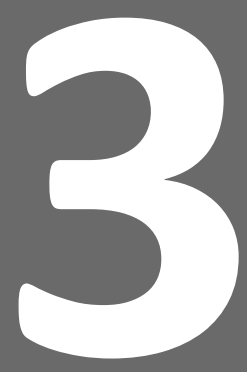

\section{The Effects of a Schoolwide} Data-Based Decision Making Intervention on Elementary School's Student Achievement Growth for
Mathematics and Spelling

This chapter is a modified version of the manuscript: Keuning, T., Van Geel, M., Visscher, A. J., \& Fox, J.-P. (submitted). The Effects of a Schoolwide Data-Based Decision Making Intervention on Elementary School's Student Achievement Growth for Mathematics and Spelling. 


\section{Abstract}

Background. Data-based decision making (DBDM) is presumed to improve student performance in primary schools in all subjects. The majority of studies in which DBDM effects has been evaluated have focused on mathematics. The limited number of other studies investigating the effects of DBDM on student performance has focused on the subject of reading.

Purpose. In the current study, the effects of an existing two-year training, in which entire school teams learned how to implement and sustain DBDM, was evaluated in 40 primary schools. Student achievement in mathematics and spelling was analyzed to broaden our understanding of the effects of this DBDM intervention.

Method. Student achievement data covering the period from August 2010 to July 2014 were retrieved from schools' student monitoring systems. Student performance on the standardized tests was scored on an ongoing ability scale per subject (mathematics and spelling), for grades one to six (students aged six to twelve years old). To investigate intervention effects on student achievement for both mathematics as well as spelling, linear mixed effect analysis was conducted.

Findings. Findings revealed a positive intervention effect for both mathematics and spelling. Furthermore, low-SES students and low-SES schools benefitted most from the intervention for mathematics. 
Throughout the last decade, policy makers around the globe have increasingly emphasized the use of data in education to enhance student achievement (Orland, 2015; Schildkamp, Ehren, \& Lai, 2012). As a result, the number of reform initiatives aimed at promoting 'databased decision making' (DBDM), or 'data-driven decision making' (DDDM) have increased rapidly (e.g. Boudett, City, \& Murnane, 2005; Carlson, Borman, \& Robinson, 2011; Love, Stiles, Mundry, \& DiRanna, 2008; Ritzema, 2015; Schildkamp, Poortman, \& Handelzalts, 2015; Slavin, Cheung, Holmes, Madden, \& Chamberlain, 2012). The idea of using student achievement data for evaluating student progress, providing tailor-made instruction, and developing strategies for maximizing performance in order to positively influence student outcomes, seems straightforward. However, the number of large-scale studies into the effects of DBDM on student outcomes is limited and the studies available have mainly focused on the effects of DBDM interventions on students' mathematics outcomes (e.g. Henderson, Petrosiono, Guckenburg, \& Hamilton, 2007; Ritzema, 2015; van Geel, Keuning, Visscher, \& Fox, 2016) rather than on reading comprehension, vocabulary or spelling. In order to broaden our understanding of the effects of DBDM on student outcomes, research into DBDM-effects on multiple subjects is necessary. A few studies have examined the effects of data use on reading (e.g. Carlson et al., 2011; Konstantopoulos, Miller, \& van der Ploeg, 2013; Quint, Sepanik, \& Smith, 2008), but to our knowledge, studies into the effects of DBDM on students' spelling outcomes are non-existent.

The University of Twente developed a DBDM intervention in which entire primary school teams were systematically introduced to DBDM and trained. Teachers learned how to analyze data, set goals, and choose appropriate instructional strategies based on the data, and, finally to alter instruction in the classroom accordingly.

In 2011, the DBDM intervention showed promising results on mathematics outcomes for a first group of 53 elementary schools. In a group of 7,500 students, a statistically significant positive improvement of student achievement, of approximately one extra month of schooling was achieved during two intervention years, was found. Furthermore, the results suggested that the intervention had been particularly effective at improving the performance of students in low socio-economic status schools (see chapter 2).

In the current study, the effects of a similar DBDM intervention on mathematics as well as on spelling achievement was studied. The contribution of this study was twofold; on the one hand it provided insights into the effects of DBDM on spelling, which as far as we know, was previously uninvestigated. On the other hand this study aimed to replicate the findings of the previous study into DBDM effects on mathematics in order to strengthen the evidence regarding the effectiveness of the intervention.

\section{Theoretical Framework}

In the following section, first the rationale underlying the assumption that DBDM positively influences student outcomes is explained. Second, we elaborate on how DBDM interventions should look like in order to improve student outcomes. Next, after a brief description of the DBDM intervention, we briefly present the results of the previous study into the effects of the DBDM intervention on mathematics. Finally, the hypotheses for this study will be presented.

\section{The Link between DBDM and Student Outcomes}

Data is supposed to inform educators, for example, for making deliberate instructional decisions, choosing a new curriculum, or for selecting a proper professional development 
intervention for their district. This data can encompass anything, from student results on benchmark assessments, student daily work, curriculum-based tests, homework, to classroom observations (Supovitz, 2012). In general, it is assumed that DBDM has a positive influence on student outcomes (Turner \& Coburn, 2012). The rationale behind this assumption can be found in the scientific evidence concerning the power of feedback. Data can provide feedback to boards or districts, schools, and teachers on how students, teachers, and schools perform in comparison to the national average, whether student progress is adequate, and on how students perform on subject matter content elements. Although feedback is not a panacea (Kingston \& Nash, 2011), the positive performance improving effects of using feedback and formative assessment have been shown in several reviews and meta-analyses (Black \& Wiliam, 1998; Fuchs \& Fuchs, 1986; Hattie \& Timperley, 2007; Hattie, 2009; Kluger \& DeNisi, 1996; Van der Kleij, Vermeulen, Schildkamp, \& Eggen, 2015).

Over the past ten years, a substantial number of studies has investigated DBDM. Several special issues regarding data use reflect the growing interest in DBDM (e.g. Coburn \& Turner, 2012; Mandinach \& Gummer, 2015; Schildkamp et al., 2012; Schildkamp \& Lai, 2013b; Turner \& Coburn, 2012). The majority of studies has focused on the effects of DBDM initiatives on teachers' attitudes, knowledge and behavior. Fewer studies have aimed at investigating student outcomes, the final criterion for DBDM-effectiveness. These studies, in which the effect of DBDM on student achievement was studied, mainly focused on mathematics and/ or reading outcomes (e.g. Carlson et al., 2011; Konstantopoulos et al., 2013; Ritzema, 2015).

To our knowledge, studies into the effects of DBDM on spelling do not exist. Spelling, however, is important for both writing and reading (Graham \& Santangelo, 2014). Especially students from a low socio-economic backgrounds run a higher risk to develop impaired spelling, consequently influencing their writing and reading skills (Graham et al., 2008). As studies into the effects of DBDM on mathematics achievement have shown that DBDM was especially beneficial for low-SES students, the intervention may yield similar benefits for spelling.

DBDM is not subject-specific, educators are supposed to apply DBDM to all subjects. To broaden our understanding of the connection between DBDM and student outcomes, interventions applied to a variety of subjects should be examined.

We will now explain why two characteristics of a DBDM intervention, regardless of subject-matter, are important for accomplishing positive student outcomes.

\section{The Challenge of Impacting Student Outcomes}

First, DBDM interventions should include all DBDM components of Figure 1 in a coherent and consistent way. Second, teachers should explicitly be involved in the intervention.

DBDM as a whole package. Data-based decision making starts with analyzing data, but it encompasses much more. As Kaufman (2014) states: "While identifying and analyzing data lays the groundwork for impactful improvements to student learning, the resulting actions and progress monitoring will ultimately determine the efficacy of DDDM efforts" (p. 341). In Figure 1, DBDM is decomposed into four components (Keuning, van Geel, Visscher, Fox, \& Moolenaar, in press). The first component, analyzing and evaluating data, is only meaningful when it is part of the entire DBDM cycle. Based on the insights gained from the analysis of data, SMART and challenging goals should be set. Next, strategies for accomplishing these goals have to be chosen and, finally, the chosen strategy should be executed. Since DBDM is ideally carried out in a systematic approach, data is also supposed to be used for monitoring 
and evaluating the effects of the implemented strategy, so that the extent to which goals have been achieved can be evaluated, and new data-informed decisions can be made.

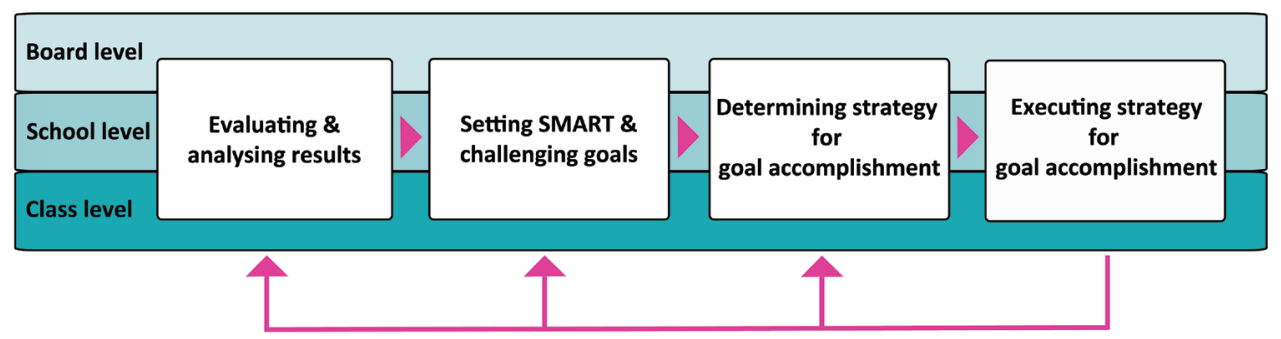

Figure 1. The DBDM cycle

As all components of the cycle are related to each other, in order for DBDM interventions to be meaningful and effective, the interventions should include all DBDM components. A school leader can produce the most impressive reports on student performance and make great plans based on these reports, but if those plans are never executed, no DBDM effects will be found. If teachers draw incorrect conclusions about students' learning needs, they are likely to implement a strategy that will not lead to the desired outcomes. Many DBDM interventions mainly focus on the first component of DBDM and have found that this does not necessarily lead to changes in teacher classroom practices, not to mention changes in student outcomes (Ikemoto \& Marsh, 2007; Marsh, Pane, \& Hamilton, 2006; Oláh, Lawrence, \& Riggan, 2010). This strongly suggests that it is important that school staff participating in the intervention learn to execute all DBDM components at a high level.

Teacher involvement in DBDM. As Figure 1 shows, the process of DBDM (ideally) takes place at the board, school and class level. Research has however repeatedly shown that, of the malleable factors within a school, teachers influence student outcomes most (Darling-Hammond, 2000; Hattie, 2009; Kaufman et al., 2014; Nye, Konstantopoulos, \& Hedges, 2004)state case study analyses, the 1993-94 Schools and Staffing Surveys (SASS. When implementing DBDM, teachers face many barriers (Wayman, Cho, Jimerson, \& Spikes, 2012), such as a lack of time, a lack of DBDM skills and knowledge, and a lack of timely and high-quality data (Marsh, 2012). As a consequence teachers may develop a negative attitude towards data use, which decreases the probability that they will use data to inform classroom practice (Wayman et al., 2012). DBDM initiatives should thus engage teachers intensively and break down the barriers for DBDM so that DBDM interventions can impact student outcomes.

Many DBDM initiatives have not involved the teacher level sufficiently. Sometimes, interventions were only implemented at the district level and teachers were unaware of their participation in a DBDM reform (e.g. McCaffrey \& Hamilton, 2007). In other cases, interventions were aimed at only training the school leader (e.g. Slavin et al., 2012) or a subset of motivated teachers (e.g. Schildkamp \& Poortman, 2015). This is often done under the assumption that a school leader or a small group of teachers will 'spread the word' throughout the entire school, but examples show that this expectation is not always fulfilled. In the so-called data-team procedure (Schildkamp \& Poortman, 2015), a group of teachers and a school leader collaboratively learn how to use data to deal with problems faced within the school. In one study, data-team results were received skeptically by other staff members who had not been involved from the outset in data team activities (Schildkamp \& Poortman, 
2015). Slavin et al. (2012) argued that "helping school leaders to understand student data is helpful but in itself does not produce educationally important gains in achievement" (p. 390).

In sum, we assume that to positively influence student achievement, a DBDM intervention should pay attention to the class/teacher level and, at that level, to the whole DBDM package, instead of only a few DBDM elements. We assume that student outcomes will improve once a DBDM intervention meets these two prerequisites, regardless of the subject the intervention focuses on. The University of Twente developed a DBDM intervention in line with these recommendations, which will be described now.

\section{The Focus Intervention}

The DBDM intervention consisted of a two-year training course for entire Dutch elementary school teams (all teachers as well as the members of the management team such as the school leader and deputy director), aimed at implementing and sustaining DBDM in the whole school organization by systematically following the DBDM cycle as shown in Figure 1.

Figure 2 provides an overview of the first and second intervention year meetings.

\begin{tabular}{|c|c|c|c|c|c|c|c|c|c|c|c|}
\hline Year 1 & 1 & 2 & 3 & 4 & $S$ & & 5 & & 6 & $S$ & 7 \\
\hline & \multicolumn{2}{|c|}{ Sept } & Oct & Nov & Dec & Jan & Febr & Mar & $A p$ & May & June \\
\hline Year 2 & & 1 & 2 & & $S$ & & 3 & & 4 & $S$ & 5 \\
\hline
\end{tabular}

Figure 2. Overview of the intervention. Numbers indicate team meetings, $S$ indicates meetings with trainer and school leader.

The first year of the intervention included seven team meetings aimed at developing DBDM knowledge and skills. The first four meetings were primarily aimed at DBDM-related knowledge and skills: analyzing and interpreting test score data from the student monitoring system ${ }^{1}$, diagnosing learning needs, setting performance goals, and developing instructional plans. Prior to the fifth meeting, teachers had executed the instructional plans in the classroom and, based on students' curriculum-based tests, classwork, homework and classroom observations, they had adjusted those plans, if necessary. At the time of the fifth meeting, the DBDM cycle had been completed for the first time and student achievement data were then discussed in a team meeting. During this meeting, teachers shared their experiences with effective and ineffective classroom practices. Meeting six focused on collaboration among team members by preparing them for observing each other's lessons; either to learn from the colleague they visited, or to provide him/her with feedback. In the last meeting of the school year, the DBDM cycle was completed for the second time and student results and classroom practices were evaluated again. Furthermore, teachers made an instructional plan for the next school year (and for the teacher(s) of that year) and also provided class information to the new teacher. In addition to the seven meetings, teachers were provided with feedback by the external trainer on both the way they had analyzed and interpreted data, as well as on the quality of their instructional plans. Furthermore, teachers were provided with a feedback

In the Netherlands over $90 \%$ of schools have a student monitoring system (SMS). Such a system includes a coherent set of tests for the longitudinal assessment of students' achievement throughout all grades of elementary education. The tests, which are usually taken twice a year (in January and in July), are available for all core subjects (mathematics, reading, spelling and vocabulary) and can best be described as interim benchmark assessments. Teachers enter students' test results into their SMS-software. Thereafter, graphs and tables representing various aspects of student performance can be retrieved from the system. The SMS-software also allows for comparison between student scores and national benchmarks. 
report concerning their teaching skills as judged by their students.

The second intervention year was aimed at deepening, sustaining and broadening DBDM within the school and included five meetings, in which new subjects were introduced (optional for schools). The DBDM cycle was completed again twice that year. Furthermore, a coaching session was included in this second school year, in which the DBDM trainer observed teachers' classroom instruction and provided them with feedback.

Aside from the two criteria for DBDM interventions discussed in the previous section (the inclusion of all DBDM-components and the intensive involvement of teachers), in the development of the intervention, the features of effective teacher professional development were also taken into account (Desimone, 2009; Van Veen, Zwart, \& Meirink, 2011). We describe these features and the method of integrating them into the intervention, in the following paragraphs.

A clear link between newly learned knowledge and skills and the practice of schools is considered essential (Timperley, 2008; Van Veen et al., 2011). Therefore, when learning how to analyze data, teachers applied what they had learned about data on their own students. Furthermore, in the instructional plans teachers learned to develop, they set goals and formulated instructional strategies to achieve these goals for their own classes.

During the meetings teachers engaged in active learning, they for example discussed their data analysis results in small groups or investigated the alignment of standardized test components and the curriculum.

Furthermore, the literature on teacher professional development emphasizes that professional development should be consistent with teachers' knowledge and beliefs. Knowledge of and beliefs surrounding DBDM varied between teachers within participating schools. During the intervention, the external DBDM coach supported schools in developing a shared DBDM vision.

Since it takes time to learn and change, duration is an important feature of effective professional development in two ways: the number of contact hours and the time span over which the TPD activity is spread (Birman, Desimone, Porter, \& Garet, 2000; Desimone, 2009; Garet, Porter, Desimone, Birman, \& Yoon, 2001). Due to the many other obligations teachers face in their work, they should be provided with sufficient time to master the learning goals (Timperley, 2008; Van Veen et al., 2011). Hence, the DBDM intervention in this study persisted for two years. The first year included seven contact meetings (each one lasted approximately four hours) and participants were encouraged to apply what they had learned in practice, for example, by carrying out data analyses, developing instructional plans, and finally, adapting their instruction (Timperley, 2008; Van Veen et al., 2011).

Finally, collective participation (e.g. as a school team) is often positively associated with active participation in professional development activities. Garet et al. (2001), Lumpe (2007), and Van Veen, et al. (2011) as well as Timperley (2008) argue that interaction and collaboration between colleagues is important for mastering and implementing an innovation. Therefore, the entire school team participated in the DBDM intervention.

By taking into account the features of effective TPD, by engaging all teachers in the training, and by paying attention to all elements of the DBDM cycle, we expected the DBDM intervention to influence teaching quality and student outcomes positively.

\section{Results of the Previous Study on the Mathematic Outcomes}

In 2011, a first group of 53 elementary schools participated in the DBDM intervention, of 
which the characteristics are described here. In the 2011-study, it was hypothesized that the intervention would lead to student achievement growth in mathematics and that this intervention effect would differ between schools. Using linear mixed models these hypotheses were confirmed, an average positive intervention effect of approximately 1.40 ability score points ( $S E=.31$ ) was found, indicating an average effect of almost one extra month of schooling during the two intervention years. This statistically significant effect was found for a group of approximately 7,500 students. The random part of the multilevel model showed that this intervention effect varied significantly across schools, whereas the correlation between the random intercept and the random intervention effect of $r=.84$ suggested that the intervention effect was smaller for schools with high initial achievement.

As expected, based on for instance TIMMS (Martin, Mullis, \& Foy, 2008), high-SES students outperformed low-SES and medium-SES students in mathematics, and boys had higher mathematics scores compared to girls. Within grades, older students scored higher than younger students. At the school level, no effects of 'urbanization' and 'school size' were found, but in line with the findings at the student level, the average school-SES was related to initial achievement: schools with few low-SES students scored significantly higher compared to medium school-SES schools and schools with many low-SES students scored significantly lower compared to medium school-SES.

It was expected that the schools with many students from a low-SES background would benefit most from the DBDM intervention: by setting ambitious goals for, and improving the achievement of all students, the probability that teachers would underestimate their low-SES students had been expected to become smaller. Interaction effects confirmed that the intervention effect was larger for schools with high proportions of low-SES students, compared to schools with few low-SES students. At the student level, a significant positive intervention effect for low-SES students compared to medium-SES students was found. Surprisingly, a positive effect was also found in favor of high-SES students compared to medium-SES students.

Finally, at the start and halfway through the second year, schools could choose to stick to mathematics or focus on a different subject (e.g. spelling or reading). It was hypothesized that the specific trajectory schools would choose would be related to the intervention effect. However, no evidence was found for this hypothesis.

In sum, the results of this study showed that a DBDM intervention, in which whole school teams are actively involved and in which attention is paid to all DBDM components, can improve students' mathematic outcomes.

\section{The Current Study}

To test whether the Focus intervention would also show similar positive effects on student outcomes for other subjects (i.e. spelling) and to strengthen the generalizability of the findings concerning mathematics, we conducted a conceptual replication (Makel \& Plucker, 2014; Schmidt, 2009) of the 2011 study.

In August 2012, a new group of 43 schools started a DBDM intervention similar to the DBDM intervention described in the previous. The same aim, implementing and sustaining DBDM in the whole school organization, was pursued by delivering a training in working with the (entire) DBDM cycle. In this intervention, the same content was taught to the participants and the training included the same sequence of meetings as the previous study. One extra classroom coaching session was added to the program in the second intervention year, to ensure that all teachers would be provided with feedback on the execution of DBDM within 
the classroom. However, the training was not led by the same DBDM trainers. These trainers had also been appointed by the University of Twente for this project and the implementation of the training was supervised by the first author of this paper, who was not directly involved in working with the schools.

The major difference between this project and the 2011 study was that it was up to the participating schools to decide whether they wanted to start the intervention with the implementation of DBDM for mathematics or spelling. In the second year, as was done in the 2011-study, schools could again choose to continue with DBDM for the subject that had been chosen in the first year or to broaden the scope to another subject. This enabled us to investigate the effects of the intervention on both mathematics and spelling.

To ensure that the training was as consistent as possible across schools and trainers, each meeting had a central topic which was the same for every participating school (e.g. analyzing test score data from the student monitoring system, goal setting and developing instructional plans, discussing student progress, and the goals for the next period). The content of these meetings was fixed for all schools, the same PowerPoint slides were used and the same assignments were done in all schools. Prior to each meeting, the trainers discussed the content for that specific meeting intensively to assure that each of them would present the information similarly. Due to the variation in school teams' prior knowledge, team members' needs and the subject chosen by a school, the time a trainer spent on a specific topic within a meeting varied somewhat between schools.

Although the content of the meetings was very similar across schools, what schools did with the information after the meetings varied and was influenced by several factors such as the characteristics of the school leader, teachers' attitudes towards the intervention, and the time that could be devoted to DBDM.

\section{Hypotheses}

In line with the 2011-study, it was expected that, as a result of the intervention, student achievement growth would increase for both mathematics (hypothesis 1A) and spelling (hypothesis 1B). Schools which did not include mathematics or spelling into their intervention were not taken into account in the analysis involved for that specific subject.

Next to these main intervention effects, it was expected that school-specific intervention effects would differ across schools (hypothesis 2). It was expected that the chosen trajectory would influence the intervention effects. Although no evidence was found to confirm this hypothesis in the previous study, we retested the hypothesis that schools that maintained the same subject for more than one year would benefit less from the intervention (hypothesis 3). The rationale for this hypothesis is that schools would mostly choose to broaden the scope of DBDM, from for example spelling to mathematics, if DBDM for spelling had been implemented successfully.

Moreover, we assumed that school-SES would partly explain differences in intervention effects between schools: in schools with a high proportion of low-SES students, the intervention effect was expected to be higher (hypothesis 4). These schools, on average, score lower than schools with a high-SES student population (Carlson et al., 2011; Inspectie van het Onderwijs, 2012) and teachers are more likely to underestimate the potential of students from a low-SES background. Since the intervention was aimed at developing ambitious goal-setting by teachers and improving the educational achievement of all students, the intervention effect was expected to be higher in low-SES schools. At the student level, the intervention effect was expected to be highest for low-SES students for the same 
Table 1. School characteristics $(N=40)$

\begin{tabular}{llcc}
\hline & & $\mathbf{N}$ & $\mathbf{( \% )}$ \\
\hline School Size & Small $(<150)$ & 13 & $(32.5 \%)$ \\
(number of students) & Medium (150-350) & 20 & $(50.0 \%)$ \\
& Large (>350) & 7 & $(17.5 \%)$ \\
Urbanization & Rural & 17 & $(42.5 \%)$ \\
& Suburban & 16 & $(40.0 \%)$ \\
& Urban & 7 & $(17.5 \%)$ \\
School-SES & High & & \\
& Medium & 12 & $(30.0 \%)$ \\
& Low & 21 & $(52.5 \%)$ \\
Main intervention subject & 7 & $(17.5 \%)$ \\
& & & \\
& Mathematics & 21 & $(52.5 \%)$ \\
& Spelling & 15 & $(37.5 \%)$ \\
& Reading & 3 & $(7.5 \%)$ \\
& Vocabulary & 1 & $(2.5 \%)$ \\
Trajectory spelling & & & \\
& No spelling at all & 12 & $(30.0 \%)$ \\
& $2^{\text {nd }}$ year spelling & 13 & $(32.5 \%)$ \\
& $1^{\text {st }}$ year spelling & 12 & $(30.0 \%)$ \\
& $>1$ year spelling & 3 & $(7.5 \%)$ \\
& & & $(12.5 \%)$ \\
Trajectory mathematics & No mathematics at all & 5 & $(35.0 \%)$ \\
& $2^{\text {nd }}$ year mathematics & 14 & $(25.5 \%)$ \\
& $1^{\text {st }}$ year mathematics & 11 &
\end{tabular}

Table 2. Student characteristics for mathematics $(n=8,396)$ and spelling $(n=6,615)$

\begin{tabular}{llcccc}
\hline & & \multicolumn{2}{c}{ Mathematics } & \multicolumn{2}{c}{ Spelling } \\
& & $\mathbf{N}$ & $\mathbf{( \% )}$ & $\mathbf{N}$ & $(\%)$ \\
\hline Gender & Boy & 4214 & $(50.3 \%)$ & 3333 & $(50.4 \%)$ \\
\multirow{3}{*}{ Student SES } & Girl & 4182 & $(49.8 \%)$ & 3282 & $(49.6 \%)$ \\
& High & 6779 & $(80.7 \%)$ & 5688 & $(86.0 \%)$ \\
& Medium & 688 & $(8.2 \%)$ & 444 & $(6.7 \%)$ \\
Number of & Low & 922 & $(11.0 \%)$ & 476 & $(7.2 \%)$ \\
observations per & 2 & 1740 & $(20.7 \%)$ & 1165 & $(17.6 \%)$ \\
student & 3 & 676 & $(8.1 \%)$ & 600 & $(9.1 \%)$ \\
& 4 & 1508 & $(18.0 \%)$ & 1095 & $(16.6 \%)$ \\
& 5 & 659 & $(7.8 \%)$ & 509 & $(7.7 \%)$ \\
& 6 & 1194 & $(14.2 \%)$ & 935 & $(14.1 \%)$ \\
& 7 & 643 & $(7.7 \%)$ & 639 & $(9.7 \%)$ \\
& 8 & 1517 & $(18.1 \%)$ & 1170 & $(17.7 \%)$ \\
& $>8$ & 459 & $(5.5 \%)$ & 502 & $(7.6 \%)$ \\
\hline
\end{tabular}


reason (hypothesis 5).

Based on large-scale studies such as TIMMS (Mullis, Martin, Foy, \& Arora, 2012), that showed that student characteristics 'gender' and 'age' were correlated with student outcomes, these variables were also taken into account in our analyses. Finally, at the school level the background variables 'school size' and 'urbanization' were included.

\section{Methodology}

Data for this study were gathered from 40 participating elementary (K-6) schools in the Netherlands from August 2012 until July 2014. Student achievement data covering the period of August 2010 until July 2014 were retrieved from the student monitoring systems of the schools. In this section, the sample and method of data collection are described first, after which a description of the data analysis methods will be presented.

\section{Sample}

In August 2012, 43 schools started with the DBDM intervention. Two schools dropped-out during the two intervention-years because of a mismatch between intervention content and school challenges at the time. In one new school, no data was gathered in the period before the intervention, as the school had not yet existed. This school was therefore excluded from this sample. The final sample included 40 participating schools. Characteristics of these schools are presented in Table 1.

Schools were supposed to first choose one subject (mathematics, spelling, vocabulary or reading), to focus on during the intervention. After one year, they were given the option to add a second subject or to continue working with only the main subject. After one and a half year schools were again given the choice to work on a new subject. This approach resulted in different intervention trajectories across schools. Five schools did not include mathematics into their trajectories and were thus excluded from analyses involving the development of mathematics achievement. For spelling, 12 schools did not include spelling and were removed from the analysis of the development in spelling results. Next, students with only one measurement point were removed from the sample. This resulted in a sample of 8,396 unique students for mathematics and 6,615 unique students for spelling. Table 2 presents the characteristics of these students.

\section{Instruments}

Student performance on the standardized tests were scored on an ongoing ability scale per subject (mathematics and spelling) for grades one to six (students aged six to twelve years old). Over the course of the two years preceding the intervention and the two intervention years, a maximum of eight measurements were observed out of eleven in total (two measurements per grade for grade years one to five and one for grade six). The total number of observations for mathematics was 42,787 and 35,361 for spelling. An overview of test occasions is depicted in Figure 3. In addition to students' ability scores, we collected student level data concerning gender, socioeconomic status (SES) category (high, medium, low) and date of birth. Age was converted on the basis of average age in months at the time of the test. 


\begin{tabular}{|c|c|c|c|c|c|c|c|c|}
\hline & \multicolumn{4}{|c|}{ Prior to intervention } & \multicolumn{4}{|c|}{ During intervention } \\
\hline & \multicolumn{2}{|c|}{ School Year 2010-2011 } & \multicolumn{2}{|c|}{ School Year 2011-2012 } & \multicolumn{2}{|c|}{ School Year 2012-2013 } & \multicolumn{2}{|c|}{ School Year 2013-2014 } \\
\hline & $\begin{array}{l}\text { Mid } \\
\text { (Febr) }\end{array}$ & $\begin{array}{l}\text { End } \\
\text { (June) }\end{array}$ & $\begin{array}{l}\text { Mid } \\
\text { (Febr) }\end{array}$ & $\begin{array}{l}\text { End } \\
\text { (June) }\end{array}$ & $\begin{array}{l}\text { Mid } \\
\text { (Febr) }\end{array}$ & $\begin{array}{l}\text { End } \\
\text { (June) }\end{array}$ & $\begin{array}{l}\text { Mid } \\
\text { (Febr) }\end{array}$ & $\begin{array}{l}\text { End } \\
\text { (June) }\end{array}$ \\
\hline Grade 3 & $x$ & $x$ & $x$ & $x$ & $x$ & $x$ & $x$ & x \\
\hline Grade 4 & $x$ & $x$ & $x$ & $x$ & $x$ & $x$ & $x$ & $x$ \\
\hline Grade 5 & $x$ & $x$ & $x$ & $x$ & $x$ & $x$ & $x$ & $x$ \\
\hline Grade 6 & $x$ & $x$ & $x$ & $x$ & $x$ & $x$ & $x$ & $x$ \\
\hline Grade 7 & $x$ & $x$ & $x$ & $x$ & $x$ & $x$ & $x$ & $x$ \\
\hline Grade 8 & $x$ & & $x$ & & x & & $x$ & \\
\hline
\end{tabular}

Figure 3. Overview of measurement occasions (shadings indicate cohorts)

\section{Multiple Single-Subject Design}

Our sample did not allow us to treat any schools as controls. However, multiple measurements prior to the intervention (baseline measurements) and multiple measurements during the intervention (treatment phase) were made to collect valuable information about school performance during and prior to the intervention. Hence, by comparing the performance of the schools from the period prior to the intervention to the period during the intervention, schools served as their own controls. Intervention effects were then calculated by means of multilevel modelling (Shadish, Kyse, \& Rindskopf, 2013; Van Den Noortgate \& Onghena, 2003). Using this so-called multiple single-subject design and fulfilling some strict conditions, it is possible to make causal inferences in studies without a control group (Kratochwill et al., 2010). Moreover, by using information of the change in performance of other schools, it was possible to increase the reliability and accuracy of the estimation of a school's intervention effect.

\section{Model}

Similar to the study in chapter 2, linear mixed effect analyses were conducted to measure changes in mathematics and spelling performance using the Ime4 package (Bates, Mächler, Bolker, \& Walker, 2014) in R (RCoreTeam, 2013). This model takes into account the nested structure of the data: measurements were nested within students and students were nested within schools. As can be seen in Figure 3, mathematics- and spelling performance was measured repeatedly over time, both before the intervention period (the control phase) and during the intervention period (the treatment phase). Growth was modeled by modeling heterogeneity in (average) student achievement while accounting for differences between measurement occasions in the different grade years in average test performance over students and schools. The differences in average achievements over grades were modeled as fixed effects, and student achievement and school achievement were allowed to vary across the general mean by introducing student and school-specific random intercepts. Random effects were introduced for average achievement over grades three to five and grades six to eight at the student level, represented as $\delta_{g i j}$. At the school level, a random effect representing the variability in the effect of the intervention across schools, represented as $\beta_{1 j}$, was introduced. This multilevel growth model can be presented as: 


$$
\begin{aligned}
& Y_{t g i j}=\mu_{t g}+\delta_{g i j}+e_{t g i j} \\
& \delta_{g i j}=\beta_{0 j}+\beta_{1 j} I_{g i j}+u_{g i j} \\
& \beta_{0 j}=r_{0 j} \\
& \beta_{1 j}=\gamma_{10}+r_{1 j},
\end{aligned}
$$

where $t$ refers to the measurement occasion, $g$ to the grade groups, $i$ the student, and $j$ the school. The multilevel growth model thus represents variation in performance between schools, grades, students, and time periods (prior to and during the intervention period). As mathematics and spelling were measured on different scales, the analyses for these two subjects were performed separately.

Interpretation of effects. The average difference between student sores at two subsequent test moments for mathematics is approximately 7.7 (Cito, 2009a) and 3.3 for spelling (Cito, 2009b). Based on the fact that there are approximately five months of schooling between two test occasions, an effect of 1.54 (mathematics) and 0.66 (spelling) can be interpreted as the expected average increase in performance due to one additional month of schooling. This effect will differ slightly between lower and upper grades, since the estimated differences in ability scores between two test occasions are larger in lower grades.

\section{Results}

In Figure 4, boxplots of the ability scores for mathematics achievement per grade are presented for the two years prior to the intervention and the two-intervention years. As would be expected, ability scores improved over a student's school career. As displayed in Figure 4, the mean ability scores prior to the intervention tended to be slightly lower, compared to mean ability scores during the intervention. Boxplots of the ability scores for spelling (see Figure 5) revealed the same trend. Note that the ability scores for spelling and mathematics were not measured on the same scale and scores are thus incomparable.

Linear mixed effect analysis provides more insight into whether the differences in mean scores indicate an intervention effect. In the following section, first the results for mathematics are given, followed by the results for spelling.

\section{Linear Mixed Effects Analysis for Mathematics}

In one school, data was entered incorrectly into the student monitoring system, as such information about the test occasions within students' school careers were not available. This school was excluded from further analysis, leading to a total of 34 schools that were included in the analysis regarding mathematics achievement.

In Table 3, the results of the subsequent models are presented. A total of seven models was analyzed. As assessed through the decrease in information criteria values (i.e., AIC, BIC, Deviance), each subsequent model was a significant $(p<.05)$ improvement over the previous one. The exception was Model 5 compared to Model 4 where 'intervention trajectory' was included as a fixed effect in the model. However, inclusion of an interaction effect of the intervention with trajectory revealed a significant improvement of the model $\left(\Delta \chi^{2}=15.90,2 \mathrm{df}, p<.001\right)$.

In the baseline-model, the subsequent tests occasions throughout a student's school 


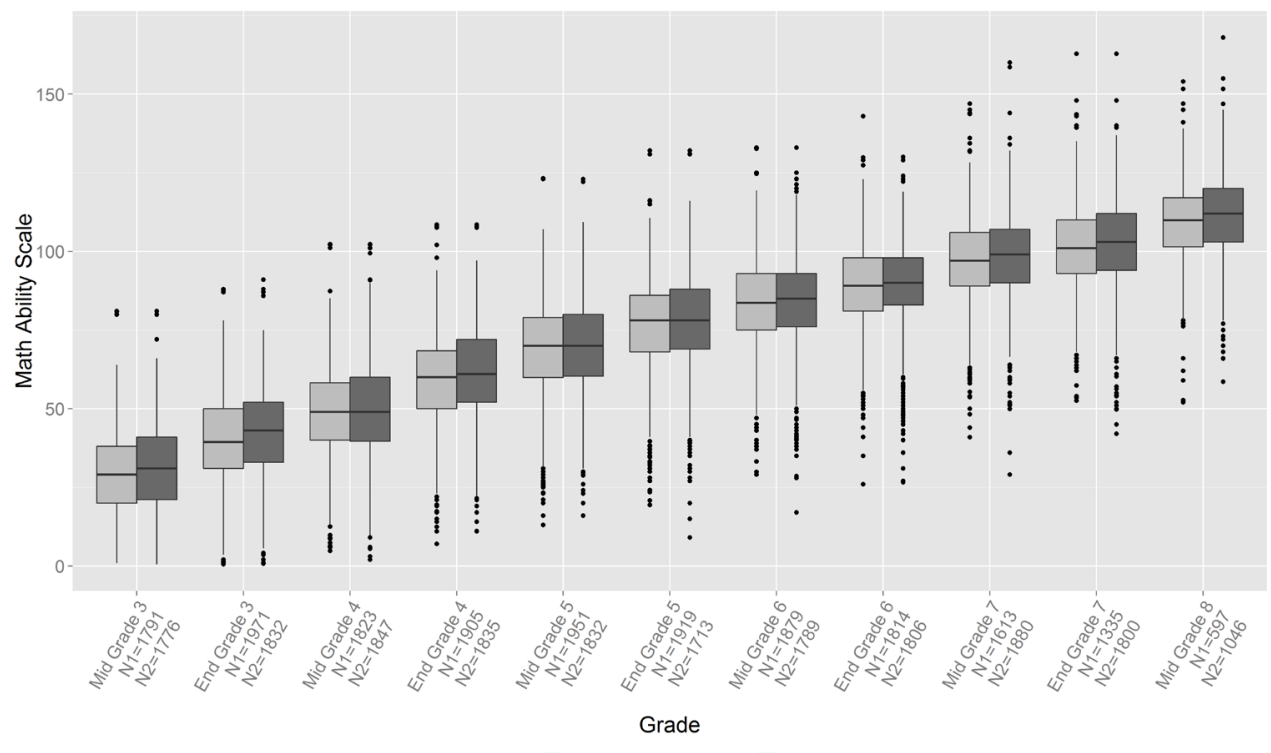

Intervention Status 追 Prior to intervention During intervention

Figure 4. Boxplots of mathematics ability scores per grade, by intervention status

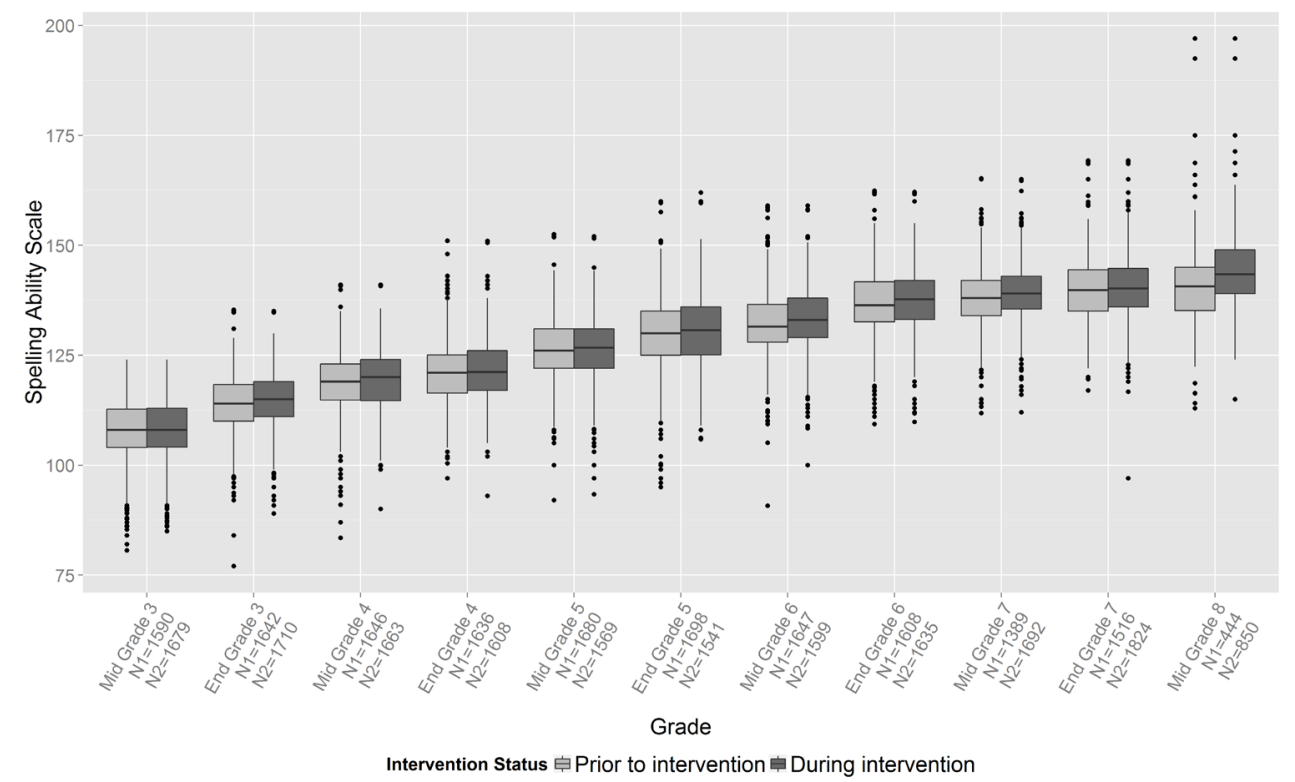

Figure 5. Boxplots of spelling ability scores per grade, by intervention status 
career were included as a fixed effect. These fixed effects showed that, in line with the boxplots in Figure 4, on average students grow in ability between every assessment. The random intercept effect at the student level (i.e. halfway through grade three) and the random effects of grades 3-5 and grades 6-8 showed considerable variation between students' mathematics achievement scores at the first assessment in grade three. This variation decreases between grades 3-5 and increases again between grades 6-8. The intraclass correlation (ICC) based on the school variance was very small $(I C C=.04)$. Note that this ICC was calculated based on the model in which the subsequent test occasions were yet included as fixed effects. More variation is explained by the cluster grade variables at the student level, where the random cluster effect 3 to 5 explained $11 \%$ of the variance in scores, and cluster 6 to 8 explained $22 \%$ of the variance in scores.

The influence of student- and school characteristics on mathematic achievement. Prior to testing the hypotheses, student characteristics were included in Model 1 and school characteristics were included in Model 2. Results indicated that high-SES students achieved higher mathematics outcomes compared to low- and medium-SES students. Moreover, boys tended to reach higher mathematics outcomes compared to boys. Regarding age, a positive effect of .46 (SE = .02) was found, suggesting that the older a student was compared to his/ her classmates, the higher his/her mathematics achievements.

At the school level, student achievement was lower in small schools compared to medium and large schools. Moreover, schools in urban and suburban areas performed poorer compared to schools in rural areas. Finally, the more low-SES students in a school, the lower the average mathematics achievement of these schools was.

The effect of the intervention on mathematics outcomes. To test the first hypothesis, concerning the effects of the intervention on student outcomes, a fixed effect of the intervention was included in Model 3. Results indicated that the general average intervention effect equaled 1.34, and differed significantly from zero, providing support for hypothesis 1 . Subsequently, in Model 4 the random effects of the intervention was included to test whether intervention effects differed between schools (hypothesis 3 ). This resulted in an increase of the fixed intervention effect to 1.39. Moreover, the random effect of 3.72 revealed that the intervention effect indeed differed between schools. Based on the $95 \%$ confidence interval of intervention effects in the population which ranges from-5.90 to 8.68 $(1.39 \pm 1.96 * 3.72)$, we can thus conclude that the effect of the intervention is not positive for all participating schools. This is graphically illustrated in Figure 6, where the random intervention effect is plotted against the random intercept. In schools placed on the right side of the 0 -axis, no effects of the intervention were observed, whereas in schools on the left side of the 0 -axis, intervention effects were positive. Note that schools on the left side of the 0 -axis prior to the intervention, generally achieved higher outcomes on mathematics compared to schools with large intervention effects. 


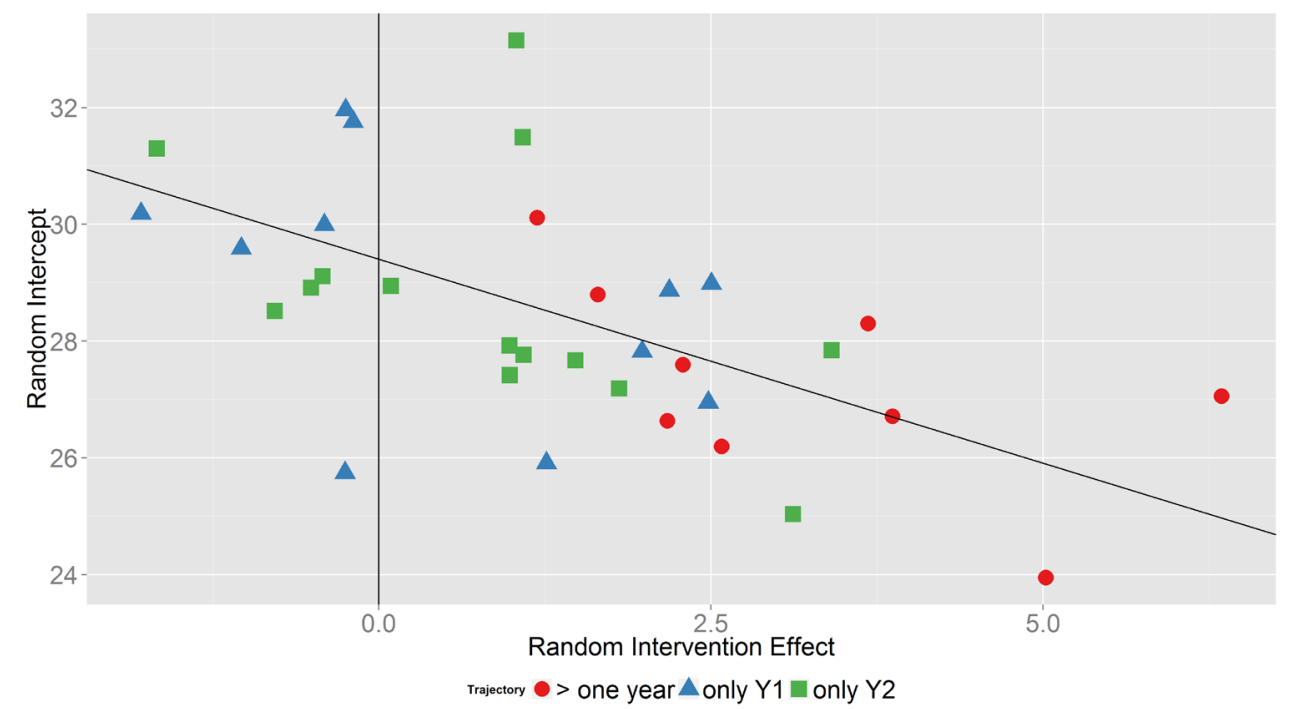

Figure 6. Random intervention effect plotted against random intercept for mathematic achievement

The influence of trajectory. For illustrative purposes, in Figure 6 schools are marked based on their trajectory. It seems that schools which focused on mathematics for more than one year showed the greatest improvement in achievement compared to schools which included mathematics only in year one or in year two. To investigate whether trajectory indeed explained part of the differences between schools (hypothesis 3), in Model 5 'trajectory' was included and in Model 6 an interaction effect of trajectory with the intervention effect was included. Although the inclusion of a main effect of trajectory did not lead to an improved model fit, according to the information criteria values $\left(\Delta \chi^{2}=5.28,2\right.$ $\mathrm{df}, p=.07)$, findings showed a significant effect of trajectory, suggesting that schools which worked on mathematics for more than one year, scored, on average, lower compared to schools which focused on mathematics in only the first year. Furthermore, Model 6 shows that the intervention effect was largest for these schools. Note that, due to the inclusion of the interaction effect with trajectory, the main effect of the intervention was no longer significant.

Interactions with student-SES and school-SES. The final two hypotheses (hypothesis 4 and 5) were about whether the school-SES and student-SES could serve as an explanation for the differences in intervention effects. A higher intervention effect was expected in schools with a high proportion of low-SES students, and for students with lowSES or medium-SES backgrounds compared to schools with high-SES backgrounds. In Model 7, these interaction effects were included. The significant interaction effect at the school level suggests that the intervention effect was larger in schools with a high proportion of low-SES students compared to schools with a high proportion of medium-SES and highSES students. This confirmed hypothesis 4. Moreover, at the individual level, both low-SES students as well as high-SES students benefitted more from the intervention compared to medium-SES students. This is not in line with our expectations, as it was not expected that 
high-SES students would also benefit more from the intervention than medium-SES students did. Thus, hypothesis 5 was only partially supported.

\section{Linear Mixed Effects Analysis for Spelling}

Results of the linear mixed effects analysis for spelling achievement are provided in Table 4. A total of 28 schools were included in the analyses of spelling achievement. Similar to the mathematics analysis, seven models were used to analyze the data. Not all subsequent models led to significant improvements.

In the baseline model, the fixed effects of the subsequent test occasions showed a similar growth pattern as the pattern shown in Figure 5 . The variance components revealed much variation at the student level, whereas the clustering of grades 3-5 explained 15\% of the total variance and the clustering of grades $6-8$ explained $31 \%$ of the total variance. The intraclass correlation at the school level was rather small: $2 \%$ of the total variance is explained at the school level, since score differences between test occasions explained much of the variance across schools.

The influence of student- and school characteristics on spelling achievement. In Model 1, student characteristics were added. Findings showed that high-SES students performed higher on spelling compared to medium-SES and low-SES students. Moreover, girls tended to achieve higher spelling scores compared to boys. Finally, similarly to mathematics achievement, older students performed better on spelling compared to their younger peers. Findings of Model 2, in which school characteristics were added, showed that spelling achievement in small schools was on average lower compared to medium sized schools. No effects of urbanization or school-SES on spelling achievements were found. Therefore, in the subsequent models, urbanization was excluded from the model. The main effect of schoolSES remained in the model in order to test hypothesis 4 in a later model.

The effect of the intervention on spelling outcomes. In order to test hypothesis 1 , a fixed intervention effect was included in Model 3. Findings showed a significant effect of .72 , suggesting that, on average, the intervention had a positive effect on spelling outcomes. To test whether this effect differed between schools, a random intervention effect was specified in Model 4, resulting in a random intervention effect of .96. By adding the random intervention effect, the fixed intervention effect slightly increased to .79. The 95\% confidence interval of intervention effects in the population ranged from-1.09 to 2.67 (.79 $\pm 1.96 * .96$ ), revealing that not all participating schools experienced positive effects. In Figure 7 , this is illustrated. As can be seen in the graph, in the majority of schools the random intervention effects were positive, but 7 out of the 28 schools did not achieve higher student achievement growth during the two intervention years. 


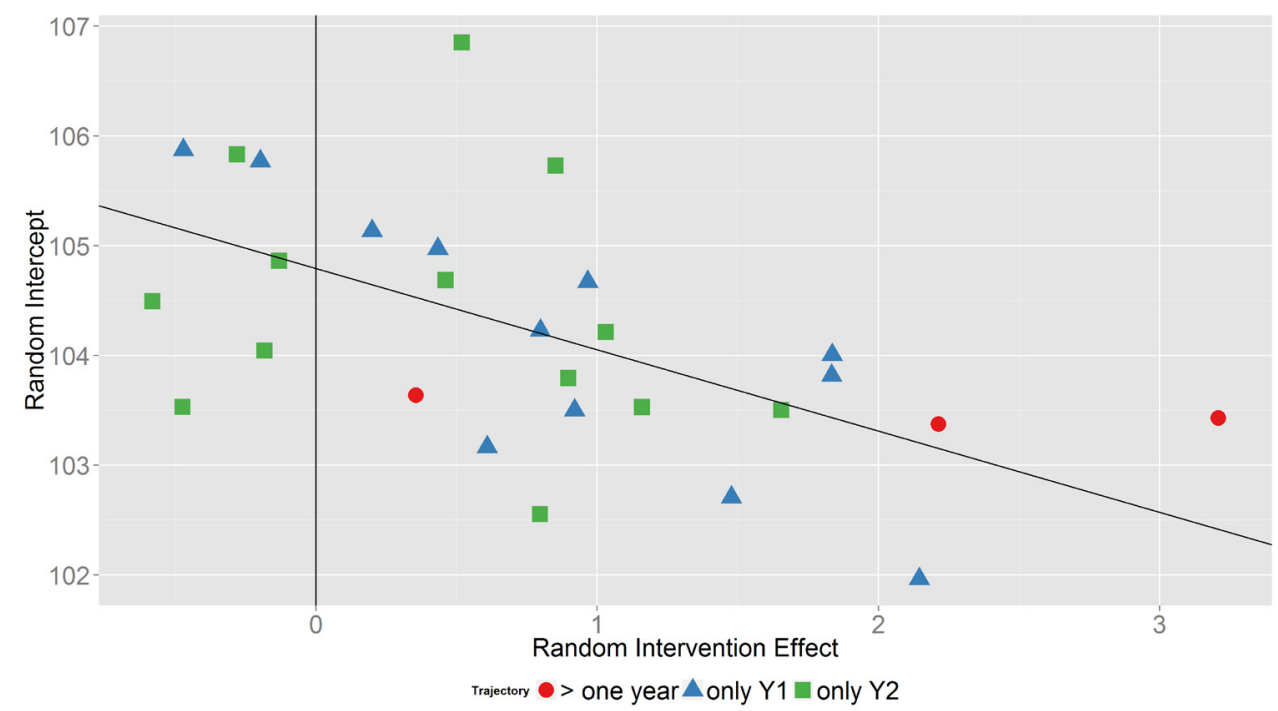

Figure 7. Random intervention effect plotted against random intercept for spelling achievement

The influence of trajectory. As can be seen in Figure 7, only three schools focused on spelling during more than one intervention year. For this reason, results from Model 5 and 6 should be interpreted with caution. In Model 5, the fixed trajectory effect was included, this effect was not significant. Furthermore, the model fit did not improve due to inclusion of trajectory. To test hypothesis 3, an interaction effect with intervention was included in Model 6. This resulted in a positive interaction effect for schools which focused on spelling for more than one year, however, note that this was based on three schools. For the three schools focusing on spelling for more than one year, the intervention effect was substantially higher than for schools which focused on spelling for only one year.

The influence of socioeconomic status. Finally, to test hypotheses 4 and 5 regarding school-SES and student-SES, interaction effects between SES and the intervention effect were included in Model 7. None of these interaction effects was significant, nor led to an improvement of the model fit. Therefore, hypotheses 4 and 5 could not be confirmed for spelling outcomes.

\section{Evaluating Model Fit}

In order to test model assumptions, several analyses were conducted. First, fitted scores and residuals at the student level (level 1 ) were plotted to check for outliers and observed scores were plotted against fitted scores to check for systematic patterns of misfit. No abnormal patterns or outliers were found in the spelling data. In the student mathematics performance data, a number of students was removed for a score of ' 0 ', since 0 was not a valid score for the test, and could thus be coded as missing.

Next, for each random effect at the first level, the ordered fitted random effect residuals were plotted against their normal quantiles in a normal QQ-plot. For both, mathematics and spelling, the random intercept effects followed an approximately normal distribution, but student level random effects, namely grades 3-5 and grades 6-8, showed 
more deviations. Both random effects seemed to be more peaked than what would be expected under the normal distribution. This means that we measured less variation in student scores in grades 3-5 and grades 6-8 than expected under the normal distribution. This can partly be explained by the fact that almost $30 \%$ of the students were measured only three or fewer times, which made it difficult to distinguish their average performance. As a result, average student performance showed less variation than expected under the normal distribution, leading to more peaked random effect distributions. Furthermore, the sample showed fewer students with more extreme average grade scores than expected under normality, but the average grade-score distribution could still be normal in the population.

At the school level (level 2), QQ-plots were examined for each random effect (random intercept and random intervention effects). For mathematics, both the random intercept residuals and the school-level random intervention effects showed some deviations. A similar pattern was found for spelling. However, there were too few schools in the sample to make inferences about the normality assumption at this level.

Finally, to test the assumption of homoscedasticity of Level-1 residual variances, the chi-square test developed by Snijders and Bosker (1999, pp. 126-127) was used. The chisquare test revealed significant results for both mathematics and spelling, indicating that at least one of the school-specific level-1 residual variances was significantly different from the other schools. Therefore, the logarithm of the estimated variances were plotted to evaluate the variation across schools. The 95\% lower and upper-bound were computed, assuming that the logarithm of residual variances were normally distributed. It became apparent that a few outliers led to a significant test result. Furthermore, the high number of students per school also led to significant differences, since many of the school-specific level-1 variances were estimated very accurately. Although this provided support for the modelling of schoolspecific residual variances, for most of the schools the assumption of a common residual variance was acceptable. 


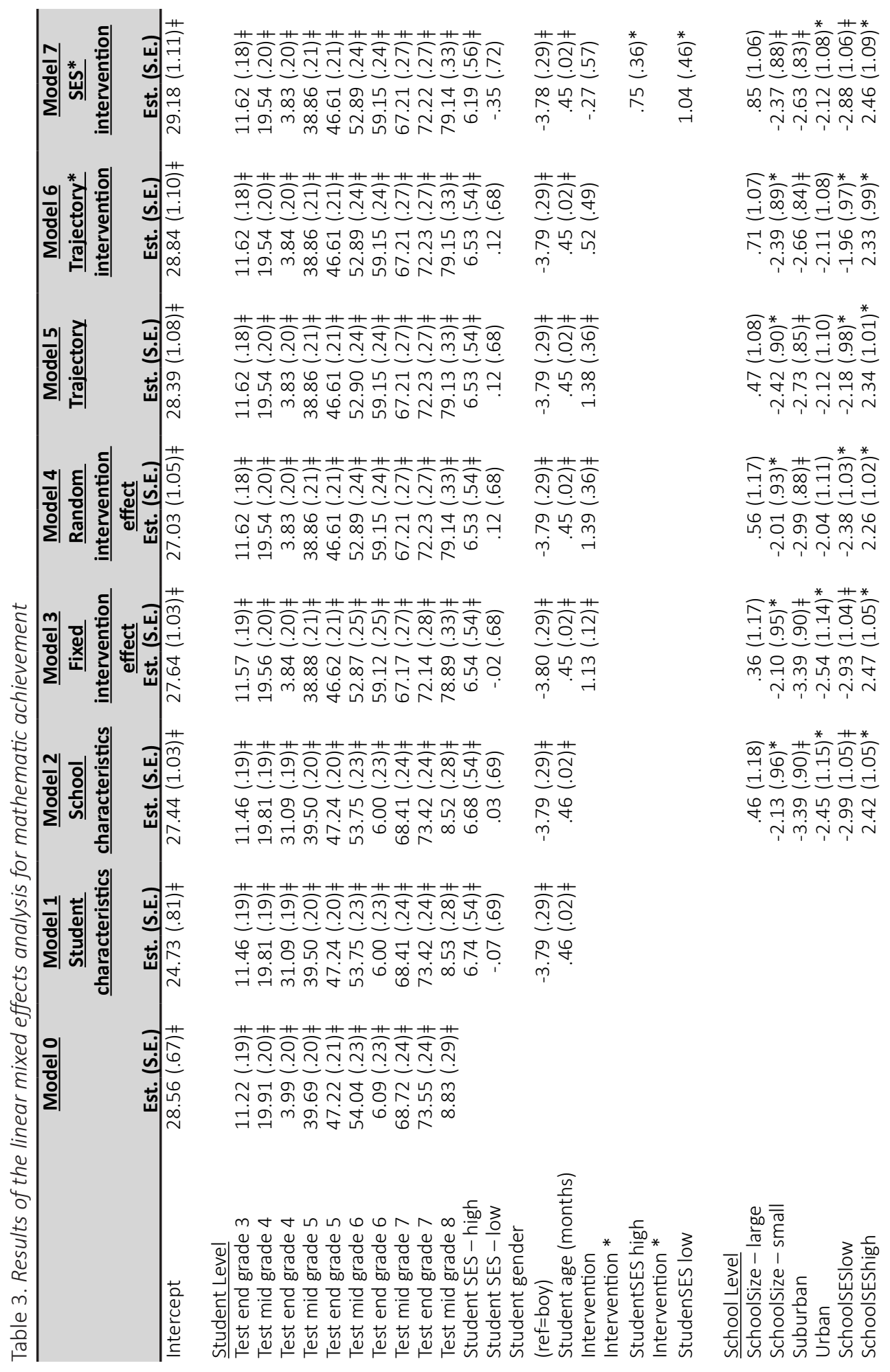




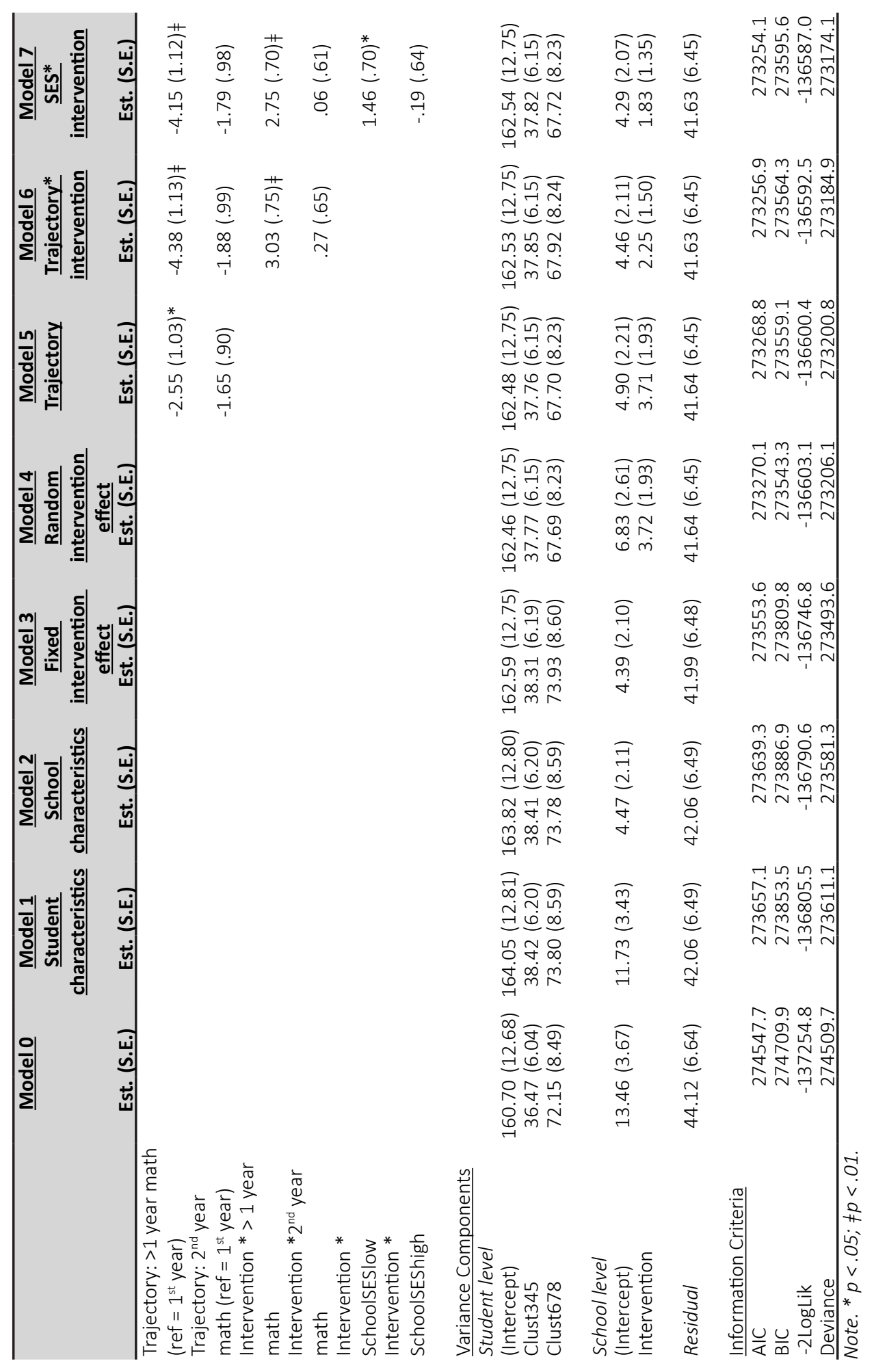




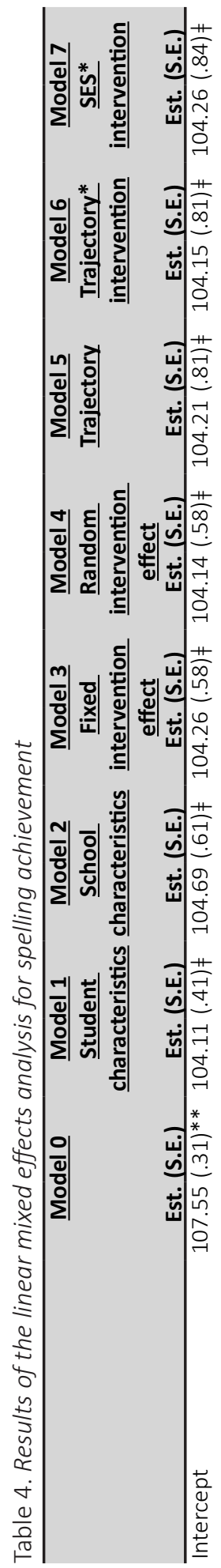

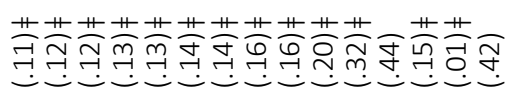

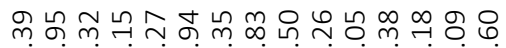

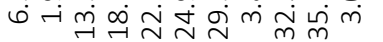

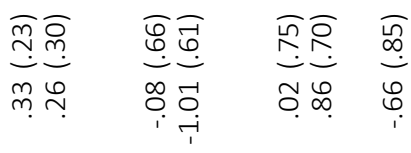

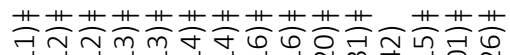

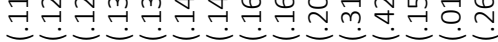

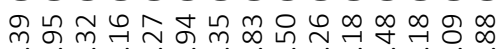

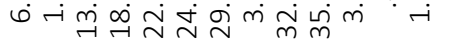

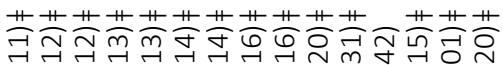

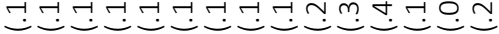

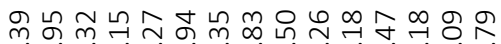

ம

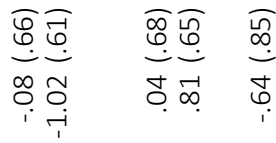

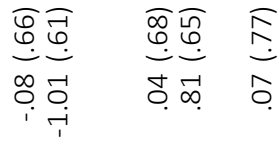

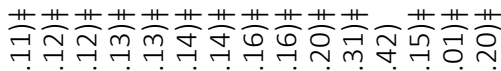

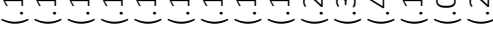

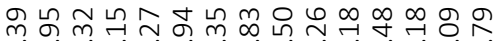

ம $\dot{\sim} \underset{\sim}{\infty} \dot{\sim} \dot{\sim} \dot{\sim} \tilde{m} \dot{m} \dot{m}$

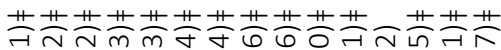

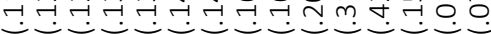

ㅇํำ

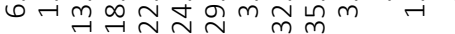

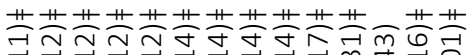

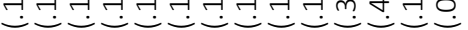

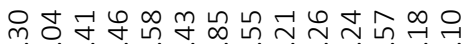

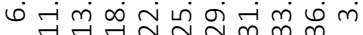

กิก สำ

ํํ ने

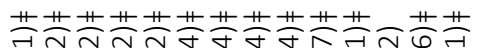

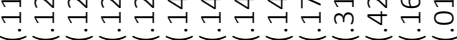

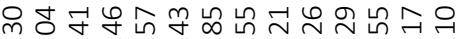

ம்

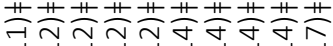

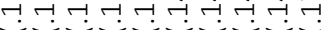

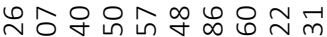

ம்

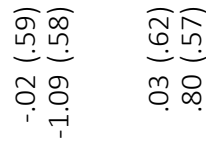

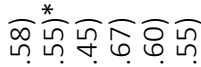

융ำ

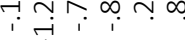

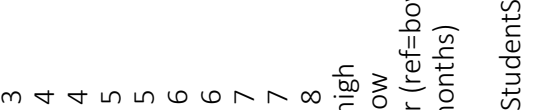

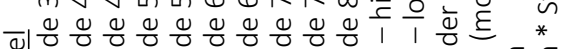

ד

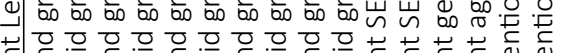

3
$\frac{3}{n}$
w
$\frac{1}{0}$
$\frac{0}{2}$
$\frac{1}{n}$

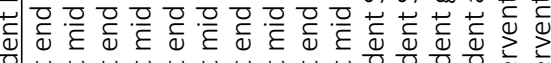

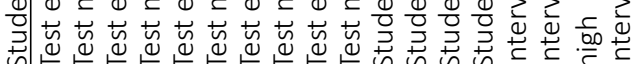

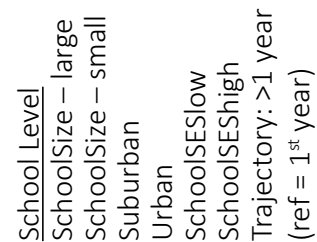




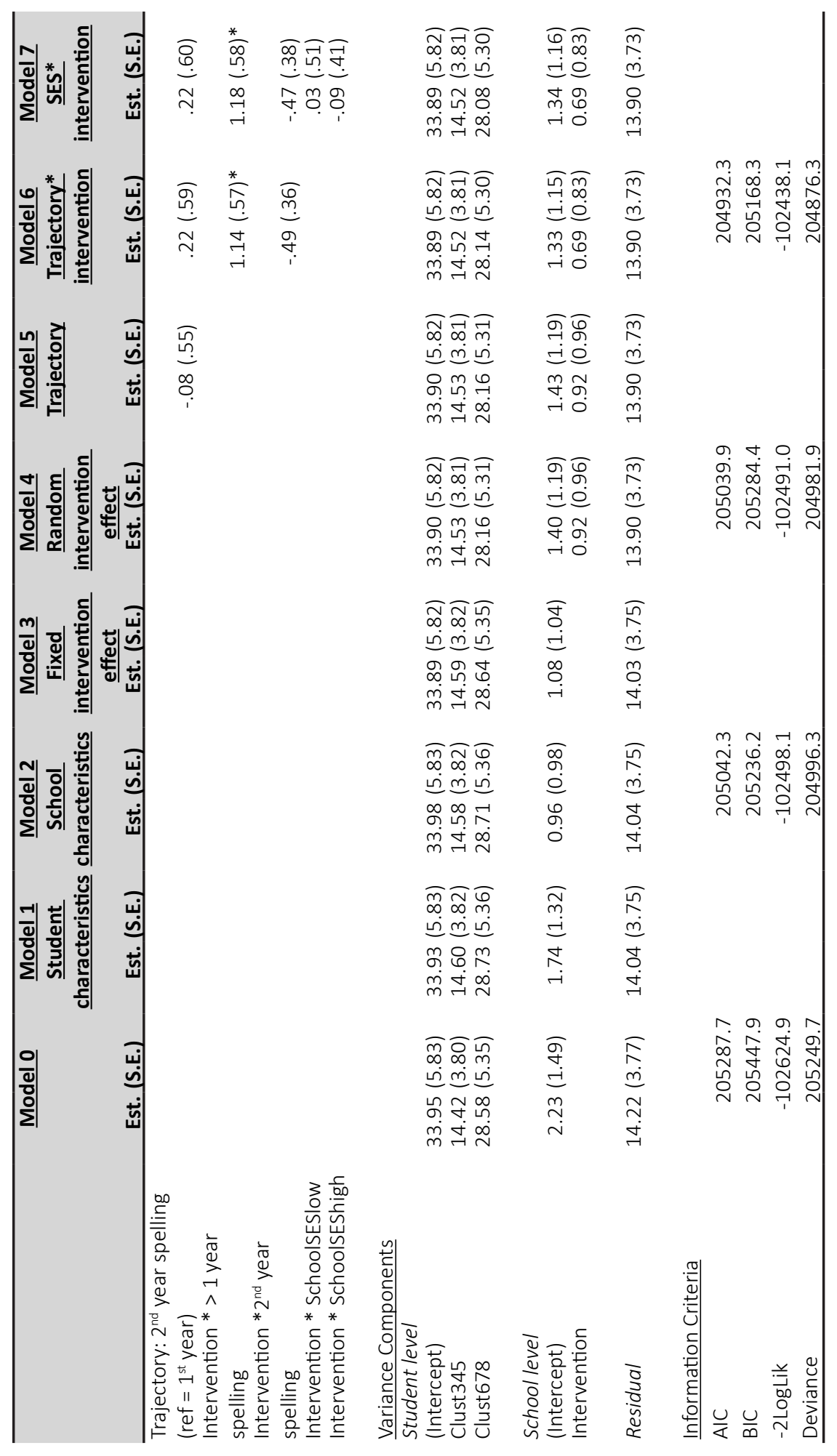




\section{Conclusions and Discussion}

Although the concept of DBDM is applicable to all school subjects, studies into the effects of DBDM on student achievement are merely aimed at mathematics or reading. In order to broaden our understanding of the link between DBDM and student performance, the effect of a DBDM intervention on both mathematics as well as spelling was studied.

We argued that to be able to influence student performance regardless of the subject-matter content, two characteristics of a DBDM intervention are specifically important. First, DBDM interventions should include all DBDM components in a coherent and consistent way. Second, interventions should take both the school level as well as the teacher level into account. In line with these criteria, we developed a two-year DBDM intervention. In a previous study, this intervention proved to be effective for mathematics. To broaden our understanding of the effectiveness of this intervention and to increase the generalizability of the findings, the effects of the intervention on student achievement were analyzed in a new cohort of 40 schools. In this study, we were not only interested in the intervention effects on mathematics achievement but also in the effects on spelling achievement.

In Table 5 the results of testing the hypotheses for both mathematics and spelling are summarized.

Table 5. Results of hypothesis testing for mathematics and spelling

\begin{tabular}{|c|c|c|}
\hline Hypotheses & Mathematics & Spelling \\
\hline $\begin{array}{l}\text { Hypothesis 1: Student achievement growth will increase as } \\
\text { a result of the intervention. }\end{array}$ & Supported & Supported \\
\hline $\begin{array}{l}\text { Hypothesis 2: School-specific intervention effects will differ } \\
\text { across schools. }\end{array}$ & Supported & Supported \\
\hline $\begin{array}{l}\text { Hypothesis 3: Schools that stick to the same subject for } \\
\text { more than one year will have the smallest intervention } \\
\text { effect. }\end{array}$ & Not supported & Not supported \\
\hline $\begin{array}{l}\text { Hypothesis 4: In schools with a high proportion of low-SES } \\
\text { students, the intervention effect will be higher }\end{array}$ & Supported & Not supported \\
\hline $\begin{array}{l}\text { Hypothesis 5: At the student level the intervention effect } \\
\text { will be highest for low-SES students. }\end{array}$ & $\begin{array}{l}\text { Partially } \\
\text { supported }\end{array}$ & Not supported \\
\hline
\end{tabular}

It was expected that student achievement growth for both mathematics as well as for spelling would be greater during the intervention, compared to student achievement growth in the two years prior to the intervention. Our findings showed that this was indeed the case for both mathematics as well as for spelling (Hypothesis 1, confirmed). On average an effect of respectively 1.39 for mathematics and .79 for spelling was found. Considering that an effect of 1.54 (mathematics) and .66 (spelling) on average can be interpreted as the expected increase in performance due to one additional month of schooling, it seems that for both subjects the intervention resulted in approximately one extra month of schooling. However, findings also revealed that the intervention effects differed between schools (Hypothesis 2, confirmed). There were schools for which the intervention effects were much larger than average. In contrast, for spelling in $25 \%$ and for mathematics in $30 \%$ of the schools, the intervention did not have an effect on student achievement growth.

We hypothesized that the trajectory schools chose to follow during the intervention 
would influence the effects of intervention. Prior to the intervention, schools could choose with which subject they wanted to start the intervention and in the second year schools could choose to focus on another subject or stick to the same subject. It was expected that schools choosing another subject in the second year DBDM would do so on the premise of successfully implementing the first subject and therefore these schools would achieve higher intervention effects. For mathematics, findings revealed effects suggesting the opposite of this hypothesis was the case (hypothesis 3 , rejected). Schools which started with mathematics and remained focused on mathematics in the second year, showed greater improvement in student performance than students from schools which changed their focus. A possible explanation might be that these schools were also the schools with the lowest mathematics starting scores. For spelling, only three schools remained focused on spelling for more than one year. Although these three schools also showed higher intervention effects on average, compared to the other schools, the small size of this group of schools offers little evidence that this holds for the spelling trajectory in general.

Furthermore, it was expected that school-SES would explain part of the differences in intervention effects across schools. For mathematics, the intervention effects were largest for schools with a large proportion of low-SES students. For spelling, the intervention effects did not significantly differ between low-SES, medium-SES or high-SES schools. Thus, hypothesis 4 was confirmed for mathematics, but not for spelling. Additionally, at the student level we expected the same trend as for hypothesis 4: students from a low-SES background would benefit most from the intervention. The results of the spelling analysis did not confirm this hypothesis. However, for mathematics we found that student outcomes improved to a greater extent for low-SES and high-SES students in comparison to medium-SES students. Therefore, hypothesis 5 was confirmed for mathematics but not for spelling.

This study confirmed the finding that mathematics outcomes improved especially for lowSES schools of the first study presented in chapter 2 . The effect sizes for mathematics are comparable to the effect sizes found in the previous study. In contrast to the first study, a significant effect of intervention trajectory was found, in favor of those schools who focused on mathematics for more than one year. It is possible that this was caused by the fact that in the current study, schools could choose their subject in the first intervention year whereas in the first study, schools were required to start with mathematics. It is likely that schools that chose mathematics performed poor on this subject, whereas in the first study schools may have performed quite well on mathematics and, as a result, showed less improvement in performance.

Moreover, this study built upon the findings of the previous study by showing that the intervention can have a positive effect on spelling. It looks like the DBDM is not subject-specific and can be implemented in multiple subjects. Interestingly, the effect of the intervention was not related to students' SES for spelling. We expected the intervention to be especially beneficial for students from low socio-economic backgrounds as these students have a higher risk of becoming poor spellers. Nevertheless, the DBDM intervention seemed to improve students' spelling outcomes regardless of their socio-economic background.

\section{Future Research}

In this study, the data did not allow us to model student performance per grade level. This would have led to a huge number of random effect parameters and a complex missing data problem, since many students were not measured more than once at each grade level. This 
problem was avoided by measuring average student performance in grades 3-5 and 6-8. As a result, we could not study differences in intervention effects across grades. In future work, it would be interesting to disentangle the average school intervention effect into grade-specific effects, to deepen our understanding of DBDM.

Furthermore, it would be interesting to conduct a retention measure in the intervention schools. As Desimone (2009) argued, it takes time to implement reform within a school and that fully changing practices in schools can take up to five years. During the two intervention years, student achievement growth improved with approximately one month of extra schooling. The question remains as to how these effects develop after these two years. A follow-up study can provide insight into the development of the effect over time: will it remain stable, increase, or decrease?

Another question raised is how we can explain the differences in effects found between spelling and mathematics. Whereas school-SES and intervention trajectory proved to explain differences in intervention effects between schools for mathematics, such relationships were not found for spelling. More research into DBDM and spelling (and especially the relationship between school-SES, trajectory and spelling achievement) is needed to, clarify these findings.

Finally, although school-SES and intervention trajectory seem to explain some of the variation in intervention effects for mathematics, a more detailed analysis of the implementation process in the participating schools could provide further insight into the reasons for these differences. The DBDM literature includes a broad range of factors that potentially explain differences in DBDM effects (Ikemoto \& Marsh, 2007; Mandinach, 2012; Schildkamp, Karbautzki, \& Vanhoof, 2014; Schildkamp \& Lai, 2013a; Schildkamp \& Poortman, 2015; Visscher \& Ehren, 2011), including teachers' attitudes, leadership, internal collaboration and the quality of the teachers. In future research the explanatory power of these factors has to be investigated in depth. 
Effects on student achievement growth for mathematics and spelling 


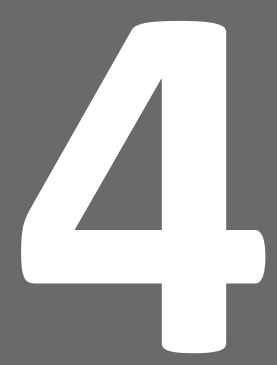

\section{Changes in Educators' Data} Literacy During a Data-Based Decision Making Intervention

This chapter is a modified version of the manuscript: Van Geel, M., Keuning, T., Visscher, A.J., Fox, J.-P. (submitted). Changes in Educators' Data Literacy During a Data-Based Decision Making Intervention 


\section{Abstract}

Background. Data literacy is assumed to be a precondition for the effective implementation of data-based decision making in schools. A two-year training course was provided at 101 primary schools. Throughout the intervention, participants acquired both knowledge and skills related to data use.

Purpose. This study was aimed at investigating changes in educators' data literacy. To investigate this, data literacy was assessed using a pretest and posttest.

Method. A multivariate multilevel IRT analysis was conducted to calibrate the test versions. The multivariate approach enabled the identification of differences in initial data literacy and data literacy development, based on educators' characteristics.

Findings. Findings showed that providing an intensive, long-term professional development trajectory, aimed at directly applying new skills, can lead to significant improvements in educators' data literacy. Academic coaches still outperformed teachers, who reached the same level of data literacy as school leaders. The 'knowledge gap' between educators with a master's degree versus higher education was closed. 
Although schools are increasingly expected to use data to guide their education, many educators do not feel prepared to use data to inform their practice (Earl \& Fullan, 2003; Ikemoto \& Marsh, 2007), struggle with the use of data (Huguet, Marsh, \& Farrell, 2014), and have shown to lack the required testing and measurement knowledge (Daniel \& King, 1998; Oláh, Lawrence, \& Riggan, 2010; Supovitz, 2012). Relatively little attention is dedicated to the preparation of educators in the use of data during their pre-service training (Mandinach, Gummer, \& Muller, 2011; Mandinach \& Gummer, 2013a; Popham, 2011). Thus, in order to develop their "human capacity to use data", professional development is essential (Mandinach \& Gummer, 2013b, p. 21).

In the Netherlands, a comprehensive intervention aimed at implementing databased decision making (DBDM) was developed and implemented in 101 primary schools. Development of participants' data literacy was stimulated throughout the intervention. This study focused on investigating changes in participants' data literacy as a result of the DBDM intervention, and at exploring differences in initial scores and the changes in scores, based on educators' characteristics.

\section{Theoretical Framework}

In the following sections, the variation in the definitions of the concept 'data literacy' is discussed. A working definition, taking the context of primary education in the Netherlands into account, is developed for the purpose of this study. This study took place within a DBDM intervention, which is described after discussing the evidence on data literacy development and its effects. The section ends with an overview of the research questions and hypotheses for this study.

\section{The Data Literacy Concept}

There is wide-spread agreement about the importance and relevance of educators being knowledgeable about testing, assessment and data, and being able to use data correctly. This is often referred to as 'data literacy', but due to a lack of a common, generally accepted definition, it remains an elusive construct (Mandinach \& Gummer, 2013b). In addition, Mandinach and Gummer (2013b) noticed that the terms 'data literacy' and 'assessment literacy' are often used interchangeably. People often seem to think of only assessment data, when talking about data in general. However, data use does not only concern assessment results, but should involve a wide range of data (Mandinach \& Gummer, 2013b).

Mandinach and Gummer (2013b) made an attempt to define data literacy by bringing together stakeholders and experts in the field of DBDM (researchers, professional development providers, funders, government representatives, policymakers and other experts), and tried to identify the skills and knowledge educators need to use data effectively to inform their practice. This convention of experts was aimed at developing an operational definition of 'data literacy'. A small majority of experts regarded assessment literacy as a part of data literacy, the other $46 \%$ indicated a large $(21 \%)$ or small $(25 \%)$ overlap between data literacy and assessment literacy, but did not provide an indication of the content that overlapped nor what would distinguish data literacy from assessment literacy (Mandinach \& Gummer, 2013b). In our search for a definition of data literacy, we therefore also investigated definitions of both data literacy and assessment literacy, in order to pinpoint similarities and differences.

Our search for a definition of data literacy led to an array of knowledge and skills that 
are assumed to be important for the effective use of data in education. Mandinach, Honey and Light (2006) stated that educators need to be able to transform raw data into actionable knowledge, and therefore that skills such as collecting and organizing data, analyzing and summarizing data, and synthesizing and prioritizing data are required. Mandinach (2012) expanded on this description of data literacy by considering the knowledge and skills required for the interpretation and use of data, and referred to this as 'pedagogical data literacy'. This definition includes the transformation of numbers, statistics and analysis outcomes into instructional strategies that meet the students' needs. Earl and Fullan (2003) stressed that the "process of human interpretation and creating meaning has to happen to change data into information and ultimately into workable knowledge" (p. 389).

Assessment literacy is often defined in a more statistical or technical manner. In their evaluation of the effects of an instructional module to enhance school personnel's assessment literacy, Zwick et al. (2008) defined it as "understanding of the psychometric and statistical principles, needed for the correct interpretation of standardized test scores" (p. 15). Interpreting test scores is a vital component of assessment literacy (Sklar \& Zwick, 2009), but Popham (2011) took a broader perspective, which includes the understanding of assessment concepts and procedures that influence educational decisions. This is also reflected in the description used by Koh (2011), in which the emphasis lies more on teachers being competent at developing and using assessment and scoring rubrics, and to master evaluative skills to judge student performance.

Based on the literature we reviewed, we concluded that there is no consensus regarding the definition of 'data literacy', due to the complexity of data use in education. However, there is a wide spread acknowledgement of the importance of using data for practice. The conceptual framework presented by Mandinach and Gummer (2013b) can be used as a guide in determining which knowledge and skills need to be combined in the construct of data literacy, and specifically placing this construct in the broader context of the data use landscape. All participants at the conference organized by Mandinach and Gummer (2013b) "clearly indicated that professional development is central to building human capacity to use data" (Mandinach \& Gummer, 2013b, p. 21). Therefore, in order to implement data use, professional development activities regarding data literacy seem essential.

\section{Defining Data Literacy for This Study}

As Mandinach and Gummer (2013b) noted, 'data literacy' cannot be defined without taking the landscape of data use into account. For the purpose of this study, we combined insights from general data literacy and assessment literature with the context characteristics of data use in the Netherlands, as a basis for a definition of 'data literacy'.

DBDM, of course, requires data to base decisions on. In order to collect such data, a variety of student assessment tools and methods are needed, ranging from teachers' daily observations to high-stakes standardized tests (Supovitz, 2012). Because the data collected by means of assessments is often used to base decisions on, the quality of the assessment tools is of great importance. In the Dutch context, a student monitoring system (SMS) has been developed by the Central Institute of Test Development (Kamphuis \& Moelands, 2000). This system includes a coherent set of tests for the longitudinal assessment of students' achievement throughout all grades of primary education. These tests, which are usually taken twice a year (in January and in July), are available for all core subjects (mathematics, reading, spelling and vocabulary). The test results are converted into an ability scale for each subject so that student progress can be monitored over grades and school years (Kamphuis \& Moelands, 
2000). Although the results of some of these tests are used for accountability purposes by the Inspectorate, the tests are clearly designed for monitoring student achievement progress and analyzing patterns in achievement across students and grades. The tests are therefore generally not perceived as 'high-stakes' tests (Kamphuis \& Moelands, 2000).

To process the collected data, tools are required to help educators organize, analyze, interpret and report data in meaningful ways (Bernhardt, 2005; Mandinach, 2012). Student monitoring system software is an example of such a technical tool. As mentioned previously, the tests in the student monitoring system enable educators to monitor students' progress throughout their entire school career (Kamphuis \& Moelands, 2000; Verhaeghe, Schildkamp, Luyten, \& Valcke, 2015). After taking a test, teachers can store the student performance data in the SMS-software. Graphs and tables representing various aspects of student performance can then immediately be retrieved from the system, and with the SMS-software it also is possible to compare the scores of students with national benchmarks.

Although there is a clear distinction between the student monitoring system (a coherent set of tests) and the SMS-software to analyze the results, in practice the term 'student monitoring system' is used for both of these tools. In the remainder of this paper, 'student monitoring system' and the abbreviation SMS are used for the set of tests as well as the software used to analyze the results.

In the definitions of data literacy by Mandinach, Honey and Light (2006) and Mandinach (2012), important aspects of data literacy are 'processing, organizing and summarizing data'. After 'clicking the right buttons', these steps are automatically performed by the student monitoring systems that are commonly used in the Netherlands. Student performance data is represented, in for example graphs or growth models, and the results can be compared with previous results of the students or the group, and can also be compared with national percentile scores. In the Netherlands, where the standardized tests and registration in SMS-software are common practice, data literacy therefore entails: knowing what kinds of analyses can and cannot be done with the system, and correctly interpreting graphs, tables, and other data representations.

\section{Data Literacy Evidence}

Although the systematic use of data is regarded as an important characteristic of effective teachers, evidence on both the development of data literacy as well as on the contribution of data literacy to student achievement is scarce. Several studies have concluded that teachers' fundamental testing and measurement knowledge is insufficient (Daniel \& King, 1998; Oláh et al., 2010; Supovitz, 2012), and that relatively little attention during their pre-service training is dedicated to the preparation of educators for the use of data (Mandinach et al., 2011; Mandinach \& Gummer, 2013a; Popham, 2011).

Empirical studies on developing data literacy points towards a similar conclusion. For example, Zwick and colleagues (2008) evaluated the effect of an instructional module about psychometric and statistical principles, and found that these modules were effective for students in teacher education preparation programs, but not for school personnel. The scores of in-service teachers, with an average experience of 11 years, were comparable to post-intervention scores achieved by pre-service teachers. Apparently, these teachers developed this knowledge during their work. On the other hand, in the study by Gotch and French (2013) no correlation was found between teaching experience and assessment literacy or assessment self-efficacy. An empirical study by Koh (2011) revealed that teachers who received ongoing, sustained professional development over the course of two school years 
instead of one-shot workshops, showed significant improvements in the use of authentic assessment, quality of assessment, and student engagement in learning. The importance of professional development to build human capacity for data use is widely acknowledged (Mandinach \& Gummer, 2013b). Therefore, a professional development intervention was developed at the University of Twente.

\section{The Intervention}

This study was conducted within the context of a comprehensive professional development intervention: a two-year training course for entire primary school teams, aimed at developing the knowledge and skills for DBDM, and implementing and sustaining DBDM in the school organization. Participants' data literacy was stimulated throughout the intervention by means of workshops on tests, scores, analyses, and by learning how to use the SMS and interpret SMS output. Furthermore, participants were required to analyze their own student performance data five times during the two intervention years by following a data analysis protocol. They received individualized feedback on the results of their analyses. Trainers also devoted time during project meetings to discuss common interpretation mistakes with the entire school team.

For this study, two cohorts of schools were used. The first cohort, of 53 schools, was exposed to the intervention in the school years 2011-12-13, the second cohort, of 48 schools, in 2012-13-14.

\section{Research Question and Hypotheses}

Since data literacy development of participants was one of the explicit aims of the intervention, the research question was: can data literacy of educators be improved, by means of an intensive DBDM intervention?

It was expected that their data literacy levels would improve during the intervention (hypothesis 1). Before DBDM was introduced, often only school leaders and academic coaches used data from a SMS to analyze student achievement. Staman, Visscher and Luyten (2014) found that initial scores for school leaders and internal coaches were significantly higher than for teachers, a finding we expect to replicate (hypothesis 2). Also, it was expected that data literacy of educators with a master's degree would initially be higher, since they would be more familiar with statistical concepts and graphical data representations as compared to educators with higher education (hypothesis 3), which is the required level of education for teachers in the Netherlands. Furthermore, it was expected that educators - especially prior to the intervention - would have benefited from each other's expertise. As such, the general level of data literacy would be higher in schools in which teams consisted of more highly educated staff. The proportion of team members that completed a master-level education was therefore expected to be positively associated with data literacy (hypothesis 4). The intervention was aimed at reaching the same level of data literacy for all participants, it was therefore expected that the 'gap' in data literacy ability, based on educational level and function (hypothesis 5 and 6) would decrease. Furthermore, scores were controlled for school background variables. Differences might be attributed to cohort effects, since half of the schools started the intervention a school year later. We also controlled for student monitoring system. 


\section{Methodology}

\section{Data Collection}

In order to determine participants' data literacy levels, a SMS data literacy test was administered at both the beginning and end of the intervention. Since three SMS software systems (C, E, and P) are commonly used by schools in the Netherlands, three test versions were developed. The tests consisted of a set of general items for all participants, and system specific questions for each of the SMS software systems. Note that all three systems are used to represent the scores on the same SMS tests, and that schools are free to choose the program they prefer. The SMS data literacy tests were developed in the summer of 2010 to be used in the first cohort of the project (not included in this study). In the tests, data literacy entails "knowing what kinds of analyses can and cannot be made with the system, and correctly interpreting graphs, tables, and other data representations". The tests therefore consisted of two parts: a) knowing which analyses can be made with the system (the 13 general data literacy items about possible analyses), and b) the correct interpretation of results from the system (the two general items about the interpretation of benchmarks, and the items about the interpretation of software specific representations). Questions were asked about the interpretation of outcomes from the analyses at the school level, the classroom level, and the individual student- level. Data literacy was regarded as one latent variable.

The 'knowing which analyses are possible' items were based on general SMS software possibilities and common (mis)conceptions about possibilities and interpretations. The two items about the general interpretation of benchmarks were meant to measure the interpretation and use of the letters indicating student performance levels, related to the national average ${ }^{1}$. For the software specific interpretation items, data representations were used that were expected to be common in DBDM practice, such as cross sections, trend analyses, and overviews of achievement growth. In Table 1, the number of general items and system specific items per SMS test version are depicted.

Table 1. Overview of the number of items per category per data literacy test version

\begin{tabular}{lccccccc}
\hline \multirow{2}{*}{ Test version } & Possibilities & $\begin{array}{c}\text { General data literacy } \\
\text { Interpreting } \\
\text { benchmarks }\end{array}$ & $\begin{array}{c}\text { System } \\
\text { specific }\end{array}$ & $\begin{array}{c}\text { Analyses } \\
\text { in system C }\end{array}$ & $\begin{array}{c}\text { Analyses } \\
\text { in system E }\end{array}$ & $\begin{array}{c}\text { Analyses } \\
\text { in system P }\end{array}$ \\
\hline SMS C & & & 2 & 15 & 0 & 0 \\
SMS E & \multirow{2}{*}{11} & 2 & 2 & 0 & 11 & 0 \\
SMS P-pre & & & 2 & 0 & 0 & 3 & 7 \\
SMS P-post & & & 0 & 0 & & 7 \\
\hline
\end{tabular}

The general items were shared across test versions. Some items were about the general possibilities of the SMS system software, for which the answer sometimes was the same across systems (for example: "With the SMS, we can compare the scores of our students with students in other countries, is this true or false?", which is false for all systems), and sometimes the correct answer differed across systems (for example: "With the SMS, we can monitor student scores for different subareas of the test, is this true or false?", which is true for systems $C$ and $E$, but false for system P). Two items were removed from the test prior to the analyses in this study, because the correct answers changed over time due to software

1 The letters indicating levels are based on the distribution of student achievement. $A=$ top $25 \%$; $B=25 \%$ above average; $C=25 \%$ below average; $D=15 \%$ far below average; $E=$ lowest scoring $10 \%$. The national average lies between level $\mathrm{B}$ and $\mathrm{C}$. 
updates. This left thirteen general data literacy items about possibilities for the analysis. Out of these thirteen items, two had different correct answers for different SMS's and were therefore treated as system specific items.

The two other general items were not related to the software system but about the general interpretation of performance levels (for example: "Margot scored a level B on the previous test and now scored a level $\mathrm{C}$. What happened to her score, relative to the national average?"). These items are labeled as 'interpreting benchmarks' in Table 1.

The items about analyses and representations in the specific systems related to software possibilities were about graphical representations of test results in the software system (for example: "Based on the trend graph above, what can you say about the scores of grade 3 in school year 2011-'12, compared to their scores in the previous school year?"). Because the specific software possibilities differed across systems, the number of items per system differed as well. The items about the interpretation of analyses in the different systems were kept as similar as possible, using representations of roughly the same information and asking the same types of questions about these analyses.

Since the graphical data representations in system $\mathrm{P}$ changed over time, two versions of this data literacy test were developed. Test version P-pre was used as a pretest, version P-post was used as a posttest. Three out of ten system specific items for system $P$ were identical in both versions.

The thirteen general items about possibilities with the systems were statements to which the respondents could respond by choosing from true, false or I don't know. The two general items about interpretation and benchmarks, and the items about analyses in the specific systems were multiple choice questions, with three or four answer choices, and the option "I don't know". Responses were scored dichotomously: 1 for a correct response and 0 for an incorrect or missing response. The response "I don't know" also was scored as zero. A translation of all data literacy test versions can be found in Appendix B.

Aside from the data literacy test, participants' background characteristics such as gender, age, and educational level, were collected by means of a questionnaire.

\section{Sample}

The data literacy pretest was conducted at the start of the intervention, in August 2011 (for cohort 1) and August 2012 (for cohort 2). Posttests were conducted at the end of the intervention - in July 2013 (cohort 1) and July 2014 (cohort 2). Out of the total number of 1883 unique respondents, 1204 (64\%) completed both the pretest and the posttest, 445 respondents (24\%) only completed the pretest, and 234 (12\%) only completed the posttest. For the purpose of this study, only respondents who completed both pretest and posttest were included. Furthermore, only schools with five or more respondents were included. This lead to a total of 1182 respondents from 83 schools. An overview of the number of respondents, and schools per system and measurement occasion can be found in Table 2 .

In Table 3, respondents' characteristics are presented. The majority of respondents were teachers and female, approximately two-third of the respondents completed higher education, which is the common educational level for primary school teachers in the Netherlands. Since only $2.4 \%$ of the respondents were older than 60 years at the beginning of the intervention, the highest age category was set to include participants aged 51 years and older. 
Table 2. Overview of final number of respondents (and schools) per system and measurement occasion

\begin{tabular}{lcccc}
\hline & & & Pretest & \\
\cline { 5 - 5 } & SMS C & SMS E & SMS P & Total \\
\hline Cohort1 & $342(24)$ & $56(3)$ & $222(18)$ & $620(45)$ \\
Cohort2 & $165(13)$ & $124(9)$ & $273(16)$ & $562(38)$ \\
Total & $507(37)$ & $180(12)$ & $495(34)$ & $1182(83)$ \\
\hline
\end{tabular}

Table 3. Overview of respondents' characteristics (final sample, $N=1182$ )

\begin{tabular}{llcc}
\hline & & $\mathbf{N}$ & $\mathbf{( \% )}$ \\
\hline Gender & Male & 167 & $(14.1 \%)$ \\
& Female & 1015 & $(85.9 \%)$ \\
Education & & & \\
(highest level) & Master's degree & 357 & $(30.2 \%)$ \\
& Higher Education & 713 & $(60.3 \%)$ \\
& Vocational Education & 103 & $(8.7 \%)$ \\
& Unknown & 9 & $(0.8 \%)$ \\
Function (highest) & & & \\
& School leader & 105 & $(8.9 \%)$ \\
& Internal coach & 93 & $(7.9 \%)$ \\
& Teacher & 928 & $(3.6 \%)$ \\
& Other & 42 & $(1.2 \%)$ \\
Une (at pretest) & Unknown & 14 & $(20.7 \%)$ \\
& & & $(24.5 \%)$ \\
& & 245 & $(22.1 \%)$ \\
& $31-40$ years & 289 & $(32.8 \%)$ \\
\hline
\end{tabular}

\section{Data Analysis}

Conceptual model. It was assumed that the data literacy test can be used to measure the unidimensional, latent ability 'SMS data literacy'. However, because the tests consisted of three parts, of which two were administered to all participants and one was SMS software specific, raw scores were neither suitable for comparing scores of users across different systems nor for comparing achievement over time. For example, it is possible that items that were specific for system $\mathrm{C}$ were easier than the items specific for system $\mathrm{P}$, for instance because the graphical representations in the former system were easier to interpret than those in the latter. Furthermore, educators were nested within schools, requiring a multilevel analysis.

Figure 1 depicts the conceptual model applied in this study. At both the individual level as well as at the school level, pretest and posttest score correlated. Covariates at the individual level were gender, age, educational level and function. At the school level, the effects of cohort, proportion of master's, and the student monitoring system used by the school were included. 

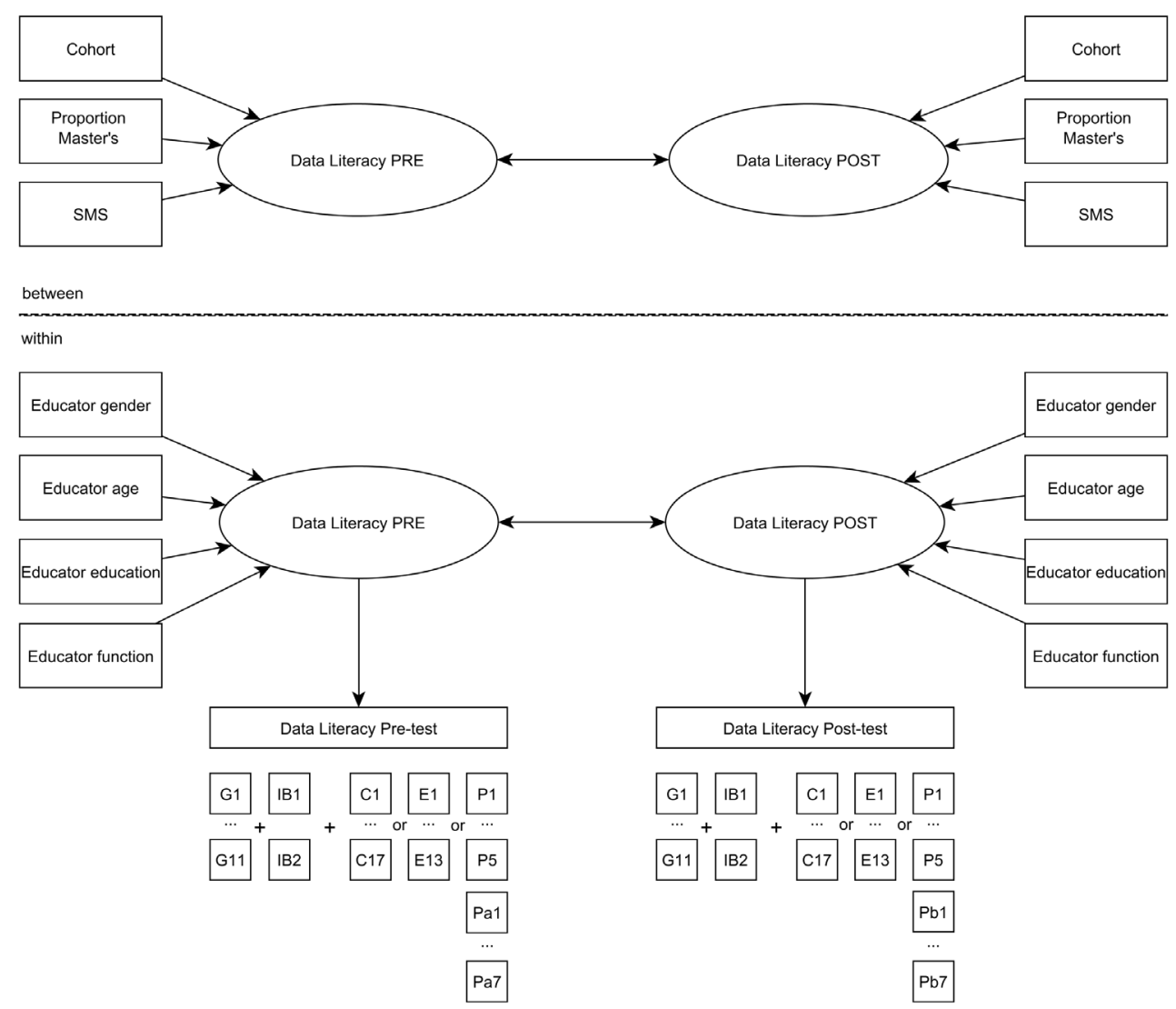

Figure 1. Conceptual model

Multivariate Multilevel Pre-Post IRT Model. In order to enable comparisons within and between respondents, take the nested structure into account and to establish different covariate effects for pretest and posttest, a multilevel IRT analysis, with a multivariate approach of the pre-post design, was conducted.

As depicted in Figure 1, the latent variable 'data literacy' was measured at the educator level, with educators nested within schools. This data structure required a multilevel analysis, since item responses were clustered within educators, who in turn were clustered within schools. Data literacy at the time of the pretest and posttest was measured using a two-parameter item response model, while accounting for the multilevel design, representing educators nested in schools, and item test differences across measurement occasions.

For the pretest, the difficulty and discrimination parameters for item $k\left(k=1, \ldots, k^{p r e}\right)$ are denoted as $b_{k}^{\text {pre }}$ and $a_{k}^{\text {pre }}$, respectively. The data literacy of educator $i$ in school $j$ at the pretest is denoted as $\theta_{i j}^{\text {pre }}$. The superscript post is used to refer to posttest characteristics. The pretest and posttest item response models were used to measure the educator's data literacy ability. The probability of a correct response to item $k$ on the pretest and the posttest, by educator $i$ of school $j$ is given by 


$$
\begin{aligned}
& P\left(Y_{i j k}^{\text {pre }}=1 \mid \theta_{i j}^{\text {pre }}, a_{k}^{\text {pre }}, b_{k}^{\text {pre }}\right)=\Phi\left(a_{k}^{\text {pre }} \theta_{i j}^{\text {pre }}-b_{k}^{\text {pre }}\right), \\
& P\left(Y_{i j k}^{\text {post }}=1 \mid \theta_{i j}^{\text {post }}, a_{k}^{\text {post }}, b_{k}^{\text {post }}\right)=\Phi\left(a_{k}^{\text {post }} \theta_{i j}^{\text {post }}-b_{k}^{\text {post }}\right),
\end{aligned}
$$

respectively. The $\Phi($.$) denotes the cumulative normal distribution function, and for each item$ response model the success probability depends on the occasion-specific item parameters and the educator's data literacy ability.

To ensure that the pretest and posttest data literacy latent variables were measured on the same scale, anchor items were selected for which parameters were fixed across measurement occasions. The anchor items were used to link the pretest and posttest scales. Furthermore, educators and schools were measured on both occasions, which led to correlated measurements at the level of educators and schools. Therefore, as depicted in Figure 1, pre- and posttest measurements were assumed to be correlated at the school level as well as the educator level. A multivariate multilevel modeling approach was defined to control for these correlations. At the level of educators, a multivariate distribution was assumed for the latent variables for the pre- and posttest measurement, which is given by

$$
\begin{aligned}
& \theta_{i j}^{\text {pre }}=\beta_{0 j}^{\text {pre }}+X_{i j}^{\text {pre }} \beta_{1}^{\text {pre }}+e_{i j}^{\text {pre }} \\
& \theta_{i j}^{\text {post }}=\beta_{0 j}^{\text {post }}+X_{i j}^{\text {post }} \beta_{1}^{\text {post }}+e_{i j}^{\text {post }}
\end{aligned}
$$

Where the educator and school-level explanatory variables are given by $X^{\text {pre }}$ and $X^{\text {post }}$ for both occasions. The error terms were assumed to be multivariate normally distributed to model the correlation between the educator's data literacy ability at the pre- and postmeasurement occasion. That is:

$$
\left(\begin{array}{c}
e_{i j}^{\text {pre }} \\
e_{i j}^{\text {post }}
\end{array}\right) \sim N\left(\left(\begin{array}{l}
0 \\
0
\end{array}\right),\left(\begin{array}{cc}
\tau_{\text {pre }}^{2} & \tau_{\theta} \\
\tau_{\theta} & \tau_{\text {post }}^{2}
\end{array}\right)\right)
$$

where the occasion-specific parameters $\tau_{\text {pre }}^{2}$ and $\tau_{\text {post }}^{2}$ represent the error variances. The parameter $\tau_{\theta}$ represents the covariance between educator's measurements, while controlling for differences by the mean terms including the explanatory variables.

The random school components $\left(\beta_{0 j}^{\text {pre }} \beta_{0 j}^{\text {post }}\right)$ describe the average performance of the school's educators at both occasions, while controlling for differences between educators and controlling for possible differences in effects of the occasion-specific explanatory variables. The random school components were also assumed to be multivariate normally distributed to address the correlation between the pretest and posttest measurements of the same school. This multivariate level-2 part is given by

$$
\left(\begin{array}{l}
\beta_{0 j}^{\text {pre }} \\
\beta_{0 j}^{\text {post }}
\end{array}\right) \sim N\left(\left(\begin{array}{l}
\gamma_{0}^{\text {pre }} \\
\gamma_{0}^{\text {post }}
\end{array}\right),\left(\begin{array}{cc}
\sigma_{\text {pre }}^{2} & \sigma_{\beta} \\
\sigma_{\beta} & \sigma_{\text {post }}^{2}
\end{array}\right)\right)
$$

where $\gamma_{0}^{\text {pre }}$ and $\gamma_{0}^{\text {post }}$ are the expected population-average scores on the pretest and posttest, respectively. The covariance matrix represents the variability in school's data 
literacy on the pretest and posttest, and $\sigma_{\beta}$ represents the covariance between pre and posttest scores at the school level.

The pre and posttest measurements are defined on the same scale, since anchor items are used to link the scales. The scale is identified by fixing the mean to zero and variance to one for the pretest. This is a common identifying restriction in multiple-group IRT modelling (Azevedo, Andrade, \& Fox, 2012; Bock \& Zimowski, 1997; Reise, Widaman, \& Pugh, 1993). Therefore, $\gamma_{0}^{\text {pre }}=0$ and the total variance of the pretest measurements is restricted to one, where the total variance is represented by the variance components at the different levels. The restriction on the total latent variance for the pretest is included in the estimation method. That is, the pretest measurements are rescaled to have a mean of zero and a variance of one during the estimation of the model parameters. Since the parameter $\gamma_{0}^{\text {pre }}$ is fixed to zero, the parameter $\gamma_{0}^{\text {post }}$ represents the average pre-post effect, representing the average score differences between the pre- and post-measurements given the explanatory variables.

The multivariate two-level model for the latent pre- and posttest measurement can be stated as a multivariate regression model with error components at different levels; that is,

$$
\left(\theta_{i j}^{\text {pre }}, \theta_{i j}^{\text {post }}\right)=\left(X_{i j}^{\text {pre }} \beta^{\text {pre }}, X_{i j}^{\text {post }} \beta^{\text {post }}\right)+\left(u_{0 j}^{\text {pre }}, u_{0 j}^{\text {post }}\right)+\left(e_{i j}^{\text {pre }}, e_{i j}^{\text {post }}\right)
$$

where the two error components at the educator and school level are multivariate normally distributed to model the correlation between educator's measurements and school measurements. The school and educator measurements are on a common scale due to the anchor items, and due to the correlation between measurements.

Schools and educators within each school were tested during the pretest and posttest. Therefore, the covariance between those measurements is complex since educators are nested in schools. The covariance between the pretest and posttest measurements of school $j$ is given by

$$
\operatorname{Var}\left(\theta_{j}^{\text {pre }}, \theta_{j}^{\text {post }}\right)=\left[\begin{array}{cc}
\Sigma_{11} & \Sigma_{12} \\
\Sigma_{21} & \Sigma_{22}
\end{array}\right]=\left[\begin{array}{cc}
\tau_{p r e}^{2} \mathbf{I}_{n j}+\sigma_{p r e}^{2} \mathbf{J}_{n j} & \tau_{\theta} \mathbf{I}_{n j}+\sigma_{\beta} \mathbf{J}_{n} \\
\tau_{\theta} \mathbf{I}_{n j}+\sigma_{\beta} \mathbf{J}_{n j} & \tau_{p o s t}^{2} \mathbf{I}_{n j}+\sigma_{p o s t}^{2} \mathbf{J}_{n j}
\end{array}\right]
$$

where $\mathbf{I}_{n_{j}}$ is the identity matrix of dimension $n_{j}$ and $\mathbf{J}_{n_{j}}$ a matrix of ones of dimension $n_{j}$. The covariance matrices $\Sigma_{11}$ and $\Sigma_{22}$ define the correlation between pretest and posttest measurements due to the nesting of educators in schools, respectively. The covariance matrix $\Sigma_{12}$, which represents the covariance between the posttest and pretest measurements of school $j$, has on the diagonal $\tau_{\theta}$ to model the correlation between each educator's pretest and posttest measurement. The non-diagonal terms describe the covariance between measurements of school $j$ at the pre- and posttest.

This multivariate multilevel modeling approach for pre- and posttest measurements extends the pre-post model of presented in chapter 5 in which a multivariate approach is applied, where only higher-level units (i.e., schools) are repeatedly measured. In this extension, pre- and posttest measurements are jointly modeled at each (hierarchical) level of the model, which means that educator and school pretest and posttest measurements are each jointly modeled. Here, the repeated measurements, made at both modeling levels, are also clustered in a cross-sectional way at each measurement occasion. Both pre-post models extend the multilevel item response model of Fox and Glas (2001) and Fox (2010).

Multivariate analysis of pre- and posttest scores has the advantage that covariates 
(such as educational level, gender and age) can be added independently at both measurement occasions. The effect therefore can also differ across occasions, and the effects of these variables at both pretest and posttest can be compared. Furthermore, in multivariate analysis the measurement errors of both measurement occasions are taken into account. Differential measurement errors are allowed at the level of schools and educators. It is also possible to include time-invariant and time-variant explanatory variables, which can differ in their effects on the pre and post measurement.

In the traditional change score method (e.g., Allison, 1990), the difference score $\beta_{0 j}^{\text {post }}-\beta_{0 j}^{\text {pre }}$ or $\theta_{i j}^{\text {post }}-\theta_{i j}^{\text {pre }}$ would be considered as a dependent variable, since the change score method is not developed for clustered outcomes. The linear regression of the change scores on the predictors ignores the differential measurement error of educator and/or school scores. This will lead to biased estimates of pre-post effects. Furthermore, when effects of explanatory variables are not invariant from the pre to posttest, they need to be included to account for differences between the two occasions. In the change score method, it is only possible to address effects of pre- posttest differences in explanatory variables, but not of the actual effects at each occasion. This complicates the interpretation of the effects of explanatory variables. Time-invariant explanatory variables will drop out of the equation, although their effects might be different for the pre and posttest.

In the so-called regressor variable approach, the pre measurement is used as a control variable, which implies that $\beta_{0 j}^{\text {post }}$ is regressed on $\beta_{0 j}^{\text {pre }}$ (or of $\theta_{i j}^{\text {post }}$ on $\theta_{i j}^{\text {pre }}$ ) and explanatory variables, to make inferences about changes in school performances. Again, the regressor variable approach has not been developed for clustered outcomes. It also follows that the measurement error associated with the pre measurement can produce biased estimates of the pre-post effect. Furthermore, in correspondence to the changes score method, the inclusion of explanatory variables is restricted to changes in the variables measured at both occasions. The regressor variable approach is implied by the multivariate model.

When considering the conditional distribution of $\theta_{i j}^{\text {post }}$ given $\theta_{j}^{\text {pre }}$, it follows that the conditionally expected measurement for educator $i$ in school $j$ at the post test given the pretest measurements of all educators of school $j$ is given by

$$
E\left(\theta_{i j}^{\text {post }} \mid \theta_{j}^{\text {pre }}\right)=X_{i j}^{\text {post }} \beta^{\text {post }}+\Sigma_{12} \Sigma_{11}^{-1}\left(\theta_{j}^{\text {pre }}-X_{i j}^{\text {pre }} \beta^{\text {pre }}\right)
$$

where the covariance between pre and post measurement is defined by the covariance between the educator's measurements but also between the school's measurements. So, beside the covariance between educator's measurements, the covariance between the school's measurements contribute to the covariance between an educator's posttest measurement and the pretest measurements of school $j$. School $j$ is measured at the pretest and the posttest, which lead to correlated measurements of school $j$. Subsequently, each educator's posttest measurement is related to all pretest measurements of school $j$.

Statistical inferences from the pre-post model. Following the procedure as described in chapter 5, Bayesian inferences can be made by computing characteristics of the posterior distribution of the model parameters. The estimated posterior means are used as estimates of the model parameters. Bayesian 95\% confidence intervals (i.e., highest posterior density (HPD) intervals) can be calculated, where each point inside the interval has a higher posterior density value than excluded points (e.g. Fox, 2010, p. 59). The 95\% HPD intervals can 
be used to evaluate whether estimated parameters are significantly different from zero ${ }^{2}$. This procedure is used to evaluate whether effects of predictor variables are significantly different from zero. Explanatory variables without a significant effect on both pre- and posttest factor scores, can be excluded from the model. Furthermore, the estimated characteristics of the posterior distribution of the difference between pretest and posttest parameters can be used to evaluate whether predictor effects differ across occasions. It follows that this procedure is specifically useful in multivariate multilevel modeling, where an interest is in the differential effects of predictors across measurement occasions.

The empty model can be used to investigate the amount of level-1 variance between educators and level-2 variance between schools. By considering the cross-sectional clustering at the pre and posttest occasion, the intra-class correlation coefficient at the pre and post measurement can be defined as

$$
\rho_{\text {pre }}=\frac{\sigma_{p r e}^{2}}{\sigma_{p r e}^{2}+\tau_{p r e}^{2}} \text {, and } \rho_{\text {post }}=\frac{\sigma_{p o s t}^{2}}{\sigma_{p o s t}^{2}+\tau_{p o s t}^{2}} .
$$

They represent the proportional amount of variance explained by the clustering of educators in schools. Following (Snijders \& Bosker, 1999, pp. 102-103) and Keuning et al., (cf. chapter $5)$, the proportional reduction in variance at each level can be computed to quantify the relevance of explanatory variables included in the model, at the level of educators or schools.

Simulation study. A Markov Chain Monte Carlo (MCMC) algorithm was developed to estimate all parameters, which was also used to obtain sampled values of functions of parameters. This was used to compute the intra-class correlations and the proportional reductions in level- 1 and level-2 variances. The MCMC algorithm is an extension of the MCMC methods for multilevel IRT models (e.g., Fox \& Glas, 2001; Fox, 2010; Stone \& Zhu, 2015) and the pre-post multivariate multilevel IRT model of Keuning et al., (cf. chapter 5).

A simulation study was carried out to investigate the parameter recovery performance of the developed MCMC algorithm. Therefore, a total of 50 data sets were simulated according to the model with true parameter values as given in Table 4. Each simulated data set consisted of 1,000 educators, nested in 100 schools, and educators' dichotomous item responses were generated for 10 items on the pre and posttest. For each data set, the MCMC algorithm was ran for 5,000 iterations, a burn-in period of 1,000 iterations was used, and the remaining sampled values from the posterior distributions were used to estimate the model parameters and standard deviations. The reported estimates in Table 4 are the pooled values, where the average is taken over the estimates corresponding to the 50 generated data sets.

It can be seen from Table 4 that the true parameter values were well recovered. It was concluded that the developed MCMC algorithm produced correct estimates for the parameters of the pre-post multivariate multilevel IRT model.

Missing data. Missing answers were scored as incorrect. Furthermore, a missing data matrix was defined, indicating missing by design for the system E and P specific items for respondents who worked at schools with system $C$, and so on for users of system $E$ and $P$. Missing data with regards to covariates were replaced with the most common value: female,

2 The (Bayesian) HPD interval provides information about the most likely parameter values given the posterior information. The true parameter value is with $95 \%$ probability likely to be different from zero when zero is not included in the parameter's 95\% HPD interval. This will be referred to as a significant effect. 
Table 4. Results simulation study for the parameter recovery of the pre post multivariate multilevel model

\begin{tabular}{|c|c|c|c|c|c|c|}
\hline & \multicolumn{2}{|c|}{$\underline{\text { True values }}$} & \multicolumn{4}{|c|}{ Estimated values (over 50 replications) } \\
\hline & \multirow[t]{2}{*}{ Pretest } & \multirow[t]{2}{*}{ Posttest } & \multicolumn{2}{|c|}{ Pretest } & \multicolumn{2}{|c|}{ Posttest } \\
\hline & & & Mean & (SD) & Mean & (SD) \\
\hline \multicolumn{7}{|l|}{ Fixed effects } \\
\hline Intercept & .00 & .05 & .00 & $(.00)$ & .04 & $(.04)$ \\
\hline Covariate 1 & 1.05 & .89 & 1.03 & $(.08)$ & .86 & $(.09)$ \\
\hline \multicolumn{7}{|l|}{$\underline{\text { Random effects }}$} \\
\hline$\overline{\text { LVl-1 variance }}$ & 1.00 & .80 & 1.02 & $(.06)$ & .80 & $(.10)$ \\
\hline Lvl-2 variance & .50 & .30 & .51 & (.09) & .31 & $(.07)$ \\
\hline Covariance Ivl-1 & .30 & & .24 & $(.04)$ & & \\
\hline Correlation Ivl-1 & .34 & & .27 & $(.04)$ & & \\
\hline Covariance $|v|-2$ & .15 & & .18 & (.05) & & \\
\hline Correlation Ivl-2 & .39 & & .45 & $(.09)$ & & \\
\hline
\end{tabular}

teacher, higher education and $\geq 51$ years old.

Inclusion of items. The first step was to determine whether discrimination and difficulty parameters were acceptable for all items. Based on an empty model with all general items set as invariant, items were removed when discrimination parameters were below .30 at all moments the item was administered (three items in total) or when the difficulty was below-3 or higher than 3 (one item). In Table 5, the final number of items per category for each test version is presented.

Table 5. Overview of the final number of items per category per data literacy test version

\begin{tabular}{|c|c|c|c|c|c|c|}
\hline \multirow[b]{2}{*}{ Test version } & \multicolumn{3}{|c|}{ General data literacy } & \multirow[b]{2}{*}{$\begin{array}{c}\begin{array}{c}\text { Analyses } \\
\text { in system C }\end{array} \\
\end{array}$} & \multirow[b]{2}{*}{$\begin{array}{c}\begin{array}{c}\text { Analyses } \\
\text { in system E }\end{array} \\
\end{array}$} & \multirow[b]{2}{*}{$\begin{array}{c}\text { Analyses } \\
\text { in system P }\end{array}$} \\
\hline & Possibilities & $\begin{array}{l}\text { Interpreting } \\
\text { benchmarks }\end{array}$ & $\begin{array}{l}\text { System } \\
\text { specific }\end{array}$ & & & \\
\hline SMS C & \multirow{4}{*}{10} & \multirow{4}{*}{2} & 1 & 15 & 0 & 0 \\
\hline SMS E & & & 1 & 0 & 11 & \multirow{3}{*}{$\begin{array}{l}7 \\
6\end{array}$} \\
\hline SMS P-pre & & & & 0 & 0 & \\
\hline SMS P-post & & & 2 & 0 & 0 & \\
\hline
\end{tabular}

Selection of anchor items. After determining the final set of items, the items that would be set as invariant across measurements were selected. It was the aim to select the five general items with the highest factor loadings as anchor items. The stability of the intended anchor items was tested by comparing item parameter estimates across different combinations of anchor items, selected from all general items. There was some variability detected in item parameter estimates over different anchor settings, which is partly caused by the sample size and sampling error. The mean estimated posterior standard deviation of the difference in item parameter estimates was approximately .10 and .11 for the discrimination and difficulty parameter, respectively. So, some item parameter differences were expected due to sampling error. The maximum difference in discriminations was approximately 0.2 . Under one anchor specification, items with a low difficulty value showed more variation in estimates, with a maximum of 0.4 . This was caused by the fact that in this setting all specified 
anchors had low discrimination values, which led to a larger difference in estimated pretest and posttest item difficulties of the non-anchor items, compared to differences obtained with anchors with higher discrimination values. Subsequently, this also led to larger differences in item difficulty estimates when comparing the estimates with those obtained under another anchor setting. As a result, the five items were considered appropriate anchor items. Item parameters of all items in the final sample are available upon request.

\section{Results}

\section{Empty Model}

The first step was to identify the empty model, in order to explain variance at the school and educator levels at the time of the pretest and posttest, and to identify overall differences in pretest and posttest scores, without taking covariates into account. The results for the empty model are presented in Table 6.

Table 6. Empty model

\begin{tabular}{|c|c|c|c|c|c|c|}
\hline & \multicolumn{3}{|c|}{ Pretest } & \multicolumn{3}{|c|}{ Posttest } \\
\hline & Estimate & SD & HPD & Estimate & SD & HPD \\
\hline \multicolumn{7}{|l|}{ Fixed effects } \\
\hline Intercept & .00 & .05 & {$[-.10, .11]$} & 1.36 & .11 & {$[1.14,1.58]$} \\
\hline \multicolumn{7}{|l|}{ Random effects } \\
\hline Level2 variance (schools) & .15 & .03 & {$[.09, .21]$} & .18 & .05 & {$[.10, .27]$} \\
\hline Level1 variance (educators) & .88 & .04 & {$[.81, .96]$} & 1.44 & .15 & {$[1.18,1.72]$} \\
\hline Intra Class Correlation & .14 & & & .11 & & \\
\hline Level1 covariance pre-post & .60 & .05 & {$[.50, .70]$} & & & \\
\hline Level1 correlation pre-post & .53 & .04 & {$[.47, .60]$} & & & \\
\hline Level2 covariance pre-post & .10 & .03 & {$[.05, .16]$} & & & \\
\hline Level2 correlation pre-post & .63 & .13 & {$[.38, .89]$} & & & \\
\hline
\end{tabular}

The nested structure of the data, with responses nested in educators, and educators nested in schools, is captured by two random effects per measurement occasion: the random effect at the school level $\left(\beta_{o j}^{\text {pre }}, \beta_{0 j}^{\text {post }}\right)$ and at the educator level $\left(\theta_{i j}^{\text {pre }}, \theta_{i j}^{\text {post }}\right)$. The random effect variances indicate that the largest part of the variance is explained at the individual level. The level-1 random effect identifies the clustering of responses within subjects.

The intraclass correlation was used to explain the proportion of variance due to the clustering of educators within schools. For the pretest, $14.4 \%$ of variance was explained by this clustering and at the posttest this was $11.0 \%$, indicating that posttest scores were less clustered at the school level. Thus, the individual level explained more of the variation in scores on the posttest.

For all models, the mean and variance of the latent scale of the pretest was fixed to identify the scale. Since the model identification was not directly implied on the intercept parameter, the estimated intercept for the pretest was $.002(S D=.05)$, and almost equal to zero. At the posttest the estimated intercept was $1.36(S D=.11)$. The estimated intercept at the posttest implies that, without controlling for background characteristics, data literacy 
ability increased compared to the pretest. On average across educators and schools, the population score on the posttest is significantly higher. For illustrative purposes, we plotted the pretest and posttest scores for four different schools in Figure 2. The diamonds represent educator scores, the dotted line represents the pre-post regression line over the entire population, and the dashed line represents the pre-post regression line for the school where the fitted area is plotted as a straight, bold line. Note that the pre-post regression lines follow from the multivariate modeling approach.

The pre-post regression slopes differ over schools. For schools with positive (negative), steep pre-post slopes, the high-scoring (low-scoring) educators on the pretest showed most improvement. So, for schools with different pretest scores, a substantially different increase in the score can be expected across schools.

\section{Adding Covariates}

In subsequent models, covariates were added step-wise, and variables with non-significant effects at both the time of the pretest and posttest were excluded from the next model. Individual-level covariates were introduced before adding school-level covariates. First, educators' educational level was added, in the next model, function was included as well. In Model 3 and 4, educators' gender and age were added subsequently. All individual-level covariates showed significant effects. In Model 5, we controlled for cohort, which appeared to be a significant covariate. Model 6 also included the student monitoring system, but this was not significant and therefore not included in Model 7, in which the proportion of masterlevel educators at the school level was added. This was also not significant, therefore Model 5 was chosen as the final model.

When comparing Model 5 with the empty model, the proportional reduction in variance at the educator level was .26 for the pretest $\left(R_{p r e, 1}^{2}\right)$, and .16 for the posttest $\left(R_{p o s t, 1}^{2}\right)$. At level 2 (school level), the proportional reduction in variance was 37 for the pretest $\left(R_{p r e, 2}^{2}\right)$ and .25 for the posttest $\left(R_{\text {post }, 2}^{2}\right)$. This indicates that the covariates explained more variance for the pretest than the posttest.

In this final model (see Table 7), the intercept at the pretest was-.08 (SD =.10) and the intercept at the posttest was $1.43(S D=.19)$, indicating a significant increase in data literacy. The intercept can be interpreted as the data literacy ability scores for male teachers, aged 41-50, with higher vocational education, working in a school from cohort 1 . Covariates can be used to explain individual differences. We will describe the effects for each covariate separately.

Education. Educators with lower vocational education scored significantly lower than higher vocational educated educators, both on the pretest as well as on the posttest. People with a master's degree scored significantly higher than intermediate vocational education on the pretest. Although the change in the effect of attaining a master's degree was not significant, the difference between educators with a higher vocational education and master's degree was no longer significant at the time of the posttest.

Function. Academic coaches and school leaders outperformed teachers on the pretest, whose scores were statistically comparable to the scores of people with 'other' functions at the school. On the posttest, academic coaches again scored significantly higher than teachers. The scores of the school leaders did not deviate from teachers' scores significantly, and this school leader effect itself was significantly smaller than at the time of the pretest.

Gender. Female educators scored lower than their male colleagues, this difference 

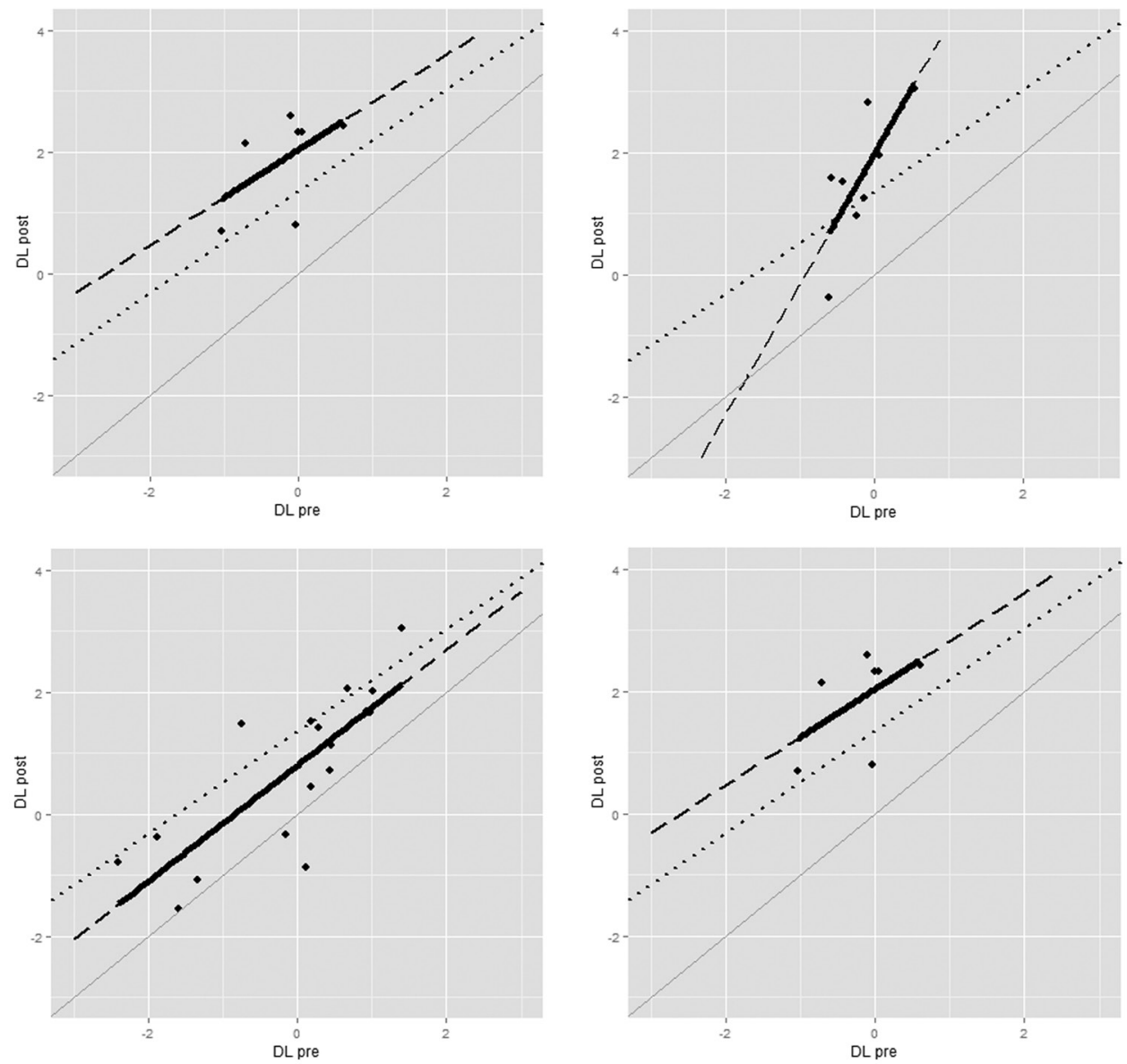

Figure 2. Pretest and posttest scores for four different schools. Reference line (grey, straight line) with intercept 0 and slope 1, dotted line represents population intercept and slope, dashed line represents intercept and slope for selected school, fitted area was printed bold

was significant on the pretest, but not on the posttest. However, the change in the effect of gender was not significant.

Age. On the pretest, only the group of educators who are 51 years or older, scored significantly lower than the reference group of 41-50 years old. This effect remained for the posttest and was even larger during that measurement (-.47 as opposed to-..24 for the pretest), but on the posttest, educators aged 30 years and younger also outperformed the age groups of $41-50$ and $\geq 51$ years old. The change in effects between the two measurement occasions was not significant for any age group.

Cohort. Educators in the second cohort scored significantly higher than educators in the first cohort. This effect was similar for both the pretest and posttest.

Conditional on the effects of the explanatory variables, the educator's and school's pretestposttest scores are correlated with a correlation of .45 and .47, respectively. So, the correlation between individual pretest-posttest scores was almost equal to the correlation between school-average pretest-posttest scores. The computed (conditional) pre-post 
regression effects (from the regression of posttest scores on pretest scores given explanatory variables) show that within schools the relationship between pretest-posttest scores was also similar to the relationship across schools. When increasing the performance on the pretest, the expected improvement on the posttest is almost identical for both educators and schools.

\section{Conclusions and Discussion}

Data literacy is one of the preconditions for the successful implementation of data-based decision making. The DBDM intervention was explicitly aimed at fulfilling preconditions in order to enable DBDM in the participating schools, and the current study was focused on investigating the effects of the intervention on the data literacy of participants.

The first hypothesis (participant's data literacy will improve during the intervention) was clearly supported, results indicated a large increase in overall participants' data literacy.

By means of multivariate multilevel latent pre-post analysis, covariate effects for pretest and posttest could be determined separately. This enabled the comparison of the covariate effects across measurement occasions and allowed us to investigate hypotheses related to 'closing the gap' based on educational level and function. Two hypotheses regarding initial data literacy were formulated. Both hypothesis 2 (data literacy of educators with a master's degree initially will be higher) and 3 (initial data literacy will be higher for school leaders and academic coaches than for teachers) were supported, this was in line with previous research (Staman et al., 2014).

At the school level, the proportion of team members that had attained a masterlevel education was expected to be positively associated with data literacy (hypothesis 4). The effect of the proportion of master-level educators was positive, but far from significant. Apparently, having a master's degree was only beneficial to those educators themselves. There were no significant differences between data literacy in schools with small and large proportions of educators with a master's degree. Hypothesis 4 therefore was rejected.

The final two hypotheses concerned an expected decrease in the effects of educational level and function (hypothesis 5 and 6). Although the changes in effects of educational level were not significant, at the posttest, there was no significant effect of having a master's degree as opposed to having followed higher vocational education. The 'data literacy gap' between attaining a master's degree and completing higher vocational education therefore seems 'closed'. Regarding function, school leader data literacy at the posttest was comparable to teacher data literacy, whereas school leaders significantly outperformed teachers on the pretest. The change in school leader effect was significant as well. The difference between teachers and academic coaches remained significant, in favor of the academic coaches.

Furthermore, differences in changes in data literacy across educators and student monitoring systems were explored. Overall, female educators showed lower data literacy scores than their male colleagues, and educators aged 51 years and older scored significantly lower than younger educators. At the posttest, educators aged 30 years and younger, outperformed their colleagues in the age groups 41-50 and 51 and older. Because three different SMS's are commonly used, it was investigated whether scores across systems differed. This was not the case; no significant differences were found across student monitoring systems.

This study showed that providing an intensive, long-term professional development trajectory, aimed at directly applying new skills in the context of participants' own school, can lead to significant improvements in educators' data literacy. Initial differences in scores, based on education and function, were not apparent on the posttest. Educators' data literacy 


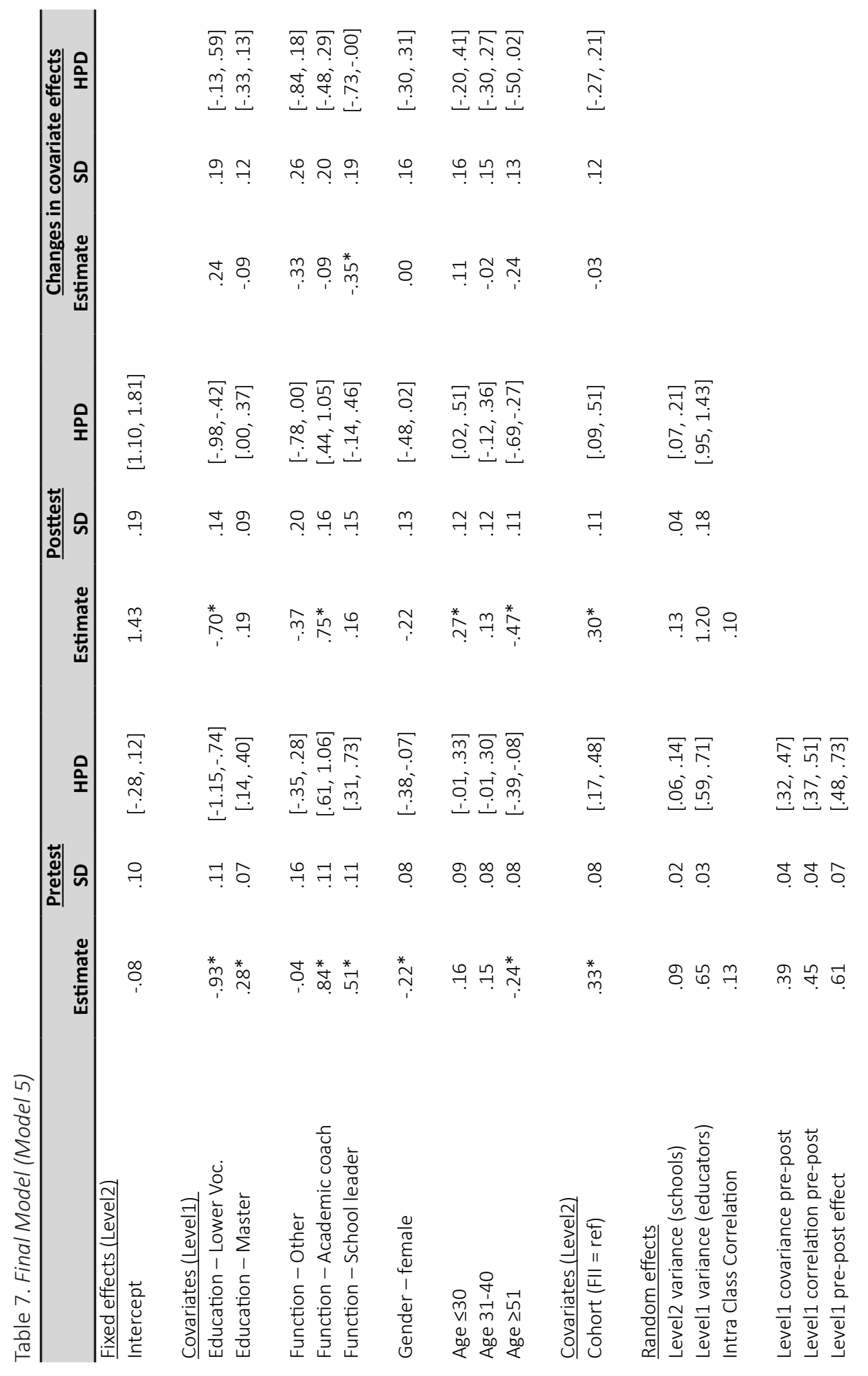


Changes in educators' data literacy

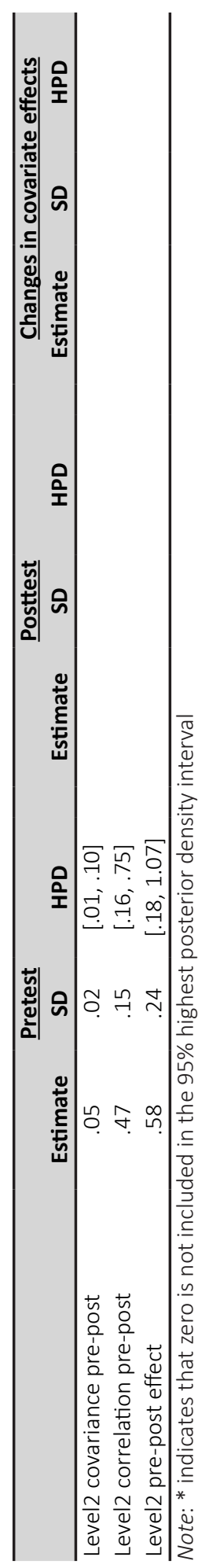


was more alike, indicating that this ability can be developed and that all educators can reach the same level.

The relevance of data literacy is widely acknowledged. In the present study, the data literacy test was used as an instrument for research purposes. In future research, it would be interesting and relevant to use the test items from this study to conduct standard-setting procedures, and to, for example, determine what would be the required starting level of data literacy for beginning teachers. The data literacy test as developed here can then be used in pre-service teacher training, to adapt their own education based on the outcomes of the tests. 
Changes in educators' data literacy 


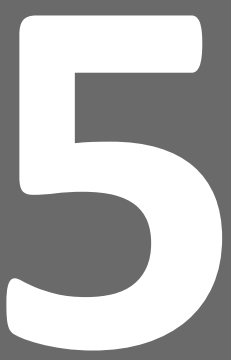

\section{The Development of Teaching Quality during a Data-Based Decision Making Intervention: A Pre-Post Multilevel IRT Approach}

This chapter is a modified version of the submitted manuscript: Keuning, T., Van Geel, M., Visscher, A.J., \& Fox, J.-P. (submitted). The Development of Teaching Quality during a Data-Based Decision Making Intervention: A Pre-Post Multilevel IRT Approach 


\section{Abstract}

Background. The successful implementation of data-based decision making (DBDM) requires high levels of teacher expertise. Although many studies into DBDM examine teachers' knowledge, skills and attitudes outside of the classroom (e.g. analyzing and interpreting data), our knowledge on how teaching skills within the classroom change during a DBDM intervention is still limited.

Purpose. In the current study, teaching quality of 209 Dutch elementary school teachers was examined as they engaged in a DBDM intervention project.

Method. In a pre-posttest design, teachers were rated by their students before the intervention and after having participated in the DBDM intervention for one year. An innovative pre-post multivariate multilevel IRT model was developed to identify whether teaching skills changed as a result of participating in the DBDM intervention.

Findings. The results indicate that teachers implemented the intended DBDM-skills in classroom practice. Students' ratings of teachers' goal-orientation improved at the posttest compared to the pretest. Suggesting that teachers to a greater extent made clear to their students what they were learning and why. Furthermore, students perceived their teachers as putting more effort in challenging students to reach their full potential. 
There is a growing international emphasis on data use in schools and educational systems, also referred to as data-based decision making (DBDM). Ikemoto and Marsh (2007) defined DBDM as "teachers, principals, and administrators systematically collecting and analyzing data to guide a range of decisions to help improve the success of students and schools" ( $p$. 108). Teachers' use of student achievement data for evaluating student progress, providing tailor-made instruction, and developing strategies for maximizing performance is considered to have a positive influence on student outcomes (Coburn \& Turner, 2012). DBDM requires a high level of teacher expertise. Teachers are for example expected to analyze and interpret student performance data, to reflect on their own functioning, and to differentiate their instruction in accordance with the variation in student progress and needs. According to the Dutch Inspectorate of Education, not all teachers in elementary schools possess the skills to implement DBDM in the classroom: approximately $40 \%$ of them does not systematically monitor student progress, and teachers in over half of all Dutch elementary schools do not differentiate instruction to meet the needs of all students (Inspectie van het Onderwijs, 2014). Research in the USA also indicates that the use of data does not necessarily imply a change in teacher practice within the classroom (Ikemoto \& Marsh, 2007; Marsh, Pane, \& Hamilton, 2006; Oláh, Lawrence, \& Riggan, 2010).

A two-year intervention for Dutch elementary (K-6) school teams was carried out, aimed at implementing and sustaining DBDM in the entire school organization. As teaching quality is essential for DBDM, improving teaching quality was one of the fundamental aims of the DBDM intervention. Students' perceptions of teaching quality in grade 2 to 6 were used to answer the following research question: To what extent does teaching quality, as measured by means of student perceptions, develop during a DBDM intervention?

\section{Theoretical Framework}

Below, we first elaborate on why teaching quality is considered important for successful implementation of DBDM, and what teaching quality in the context of DBDM entails. Next, a brief description of the DBDM intervention, of which the effects were studied, is presented in order to explain how elements of this intervention were meant to improve teaching quality. Thirdly, we explain why teaching quality in this study was measured by means of student perceptions. Finally, the hypotheses will be presented and we will elaborate on how changes in teaching quality during an intervention can be modelled using a pre-post multivariate multilevel IRT model.

\section{Teaching Quality in the Context of DBDM}

DBDM can take place at all levels of the educational system: the teacher level, the school level and the board or district level. In this study we focused on the implementation of DBDM at the teacher level. Teachers working within the framework of DBDM use data to monitor their students' strengths and weaknesses, set goals that match with the performance levels achieved, and determine strategies to achieve those goals within their own classroom. In other words, it is no longer possible to simply let the textbook determine when particular subject matter content is taught and how. Some students will need additional challenge because they have already grasped subject matter, while other students would rather benefit from additional instruction. Thus, teachers are required to adjust student learning objectives on the basis of evidence about their students' needs, and to adapt their instructional strategies accordingly, and to do this for a group of students who vary in their progress and needs. 
Studies of teachers' roles in the context of DBDM mainly focus on the skills that teachers need to master outside of the classroom, such as the way teachers analyze and interpret data (Mandinach, 2012; Marsh, 2012). However, as Anderson, Leithwood and Strauss (2010) stated: "It is not data use per se that affects the quality of teaching and learning; rather it is the appropriateness of actions actually taken based on data-informed decisions about the nature of the problem and how it might be solved" (p. 321). Therefore, in this study we focus on skills teachers need to master within the classroom. We consider the following five teaching skills to be important for ensuring effective implementation of DBDM within the classroom. The first three skills, 'creating a safe climate', 'effective classroom management' and 'providing clear instruction', can be considered as basic teaching skills (Fauth, Decristan, Rieser, Klieme, \& Büttner, 2014; Pianta, Hamre, Haynes, Mintz, \& La Paro, 2007). In addition to these basic skills, two other teaching skills are important for DBDM: challenging students and teaching in a goal-oriented way. Below, all these skills are described more in detail.

- Creating a safe climate. Being able to create a safe climate for learning is one of the most important teaching skills, if not the most important prerequisite for learning in general. There is empirical evidence of a positive relationship between a safe classroom climate and student motivation (Hamre \& Pianta, 2006), and their learning outcomes (Brophy, 2000; Cornelius-White, 2007; Hattie, 2009). A safe classroom is characterized by a safe and supportive environment, expressed by a positive relationship between students and teachers (Fauth et al., 2014; Ferguson, 2012; Kyriakides, 2005). Students need to feel free to ask questions and teachers' responses to those questions should be supportive. Furthermore, teachers should have positive expectations of students and support students' self-confidence (Van de Grift, 2014).

- Classroom management. Efficient classroom management is considered to be a precondition for time-on-task (Fauth et al., 2014) which in turn has a positive effect on students' learning outcomes (Seidel \& Shavelson, 2007). In well-managed classrooms lessons start on time, there are clear classroom rules and procedures, students pay attention when the teacher explains subject matter, and it is quiet when students work individually (Ferguson, 2012; Kyriakides, 2005). In such classrooms little time is lost during lesson changes and maintaining order (Van de Grift, 2014).

- Providing clear instruction. Explaining content in such a way that students understand it can be considered to be the heart of teaching (Danielson, 2007). A teacher must be able to clearly explain learning material to students (Creemers, Kyriakides, \& Antoniou, 2013) and assess whether or students understand the new material through students questions (Van de Grift, 2014). Ferguson (2012) stated that "the most effective teachers should be able to diagnose students' skills and knowledge, and they need multiple ways of explaining ideas that are likely to be difficult for students to grasp" (p. 26).

- Challenging students. Since the aim of DBDM is to optimize achievement of all students in the school, the teacher should challenge all students in order to reach their full potential. Ferguson (2012) argued that challenging teachers "tend to monitor students' effort and confront students if their effort is unsatisfactory. Students who do not devote enough time to their work, or who give up too easily in the face of difficulty are pushed to do more. Similarly, students who do not think deeply, or who resist reasoning their way through challenging questions are both supported and pushed." (p. 26). 
- Goal-oriented teaching. An essential part of DBDM is setting goals based on data (Visscher \& Ehren, 2011). Although goal-setting in particular is an activity carried out outside the classroom, it is desirable to pursue the goals set in the classroom. To make lessons more meaningful for students and to encourage their active participation (Kyriakides, 2005), it should be clear to students what they are learning and why. Teachers therefore need to clarify the lesson objectives at the start of the lesson (Locke \& Latham, 2002). Morisano and Locke (2013) argued that feedback to monitor goal progress is essential, teachers thus are also supposed to provide students with feedback on their work.

The quality of the afore mentioned teaching skills is essential in order to achieve the ultimate goal of DBDM: increased student achievement (Kaufman, Graham, Picciano, Popham, \& Wiley, 2014). During the implementation of DBDM, special attention therefore needs to be devoted to teacher practice within the classroom. In the current study the effects of a DBDM intervention on the five teaching skills were evaluated. In the following section, the features of the DBDM intervention are presented.

\section{The DBDM Intervention}

Similar to a number of DBDM interventions in the USA (e.g. Carlson, Borman, \& Robinson, 2011; Love, Stiles, Mundry, \& DiRanna, 2008; Slavin, Cheung, Holmes, Madden, \& Chamberlain, 2012), the DBDM intervention in this study was a two-year training course for entire elementary school (K-6) teams in the Netherlands, aimed at implementing and sustaining DBDM throughout the school organization. A fundamental component of the intervention was the 'DBDM-cycle' as depicted in Figure 1.

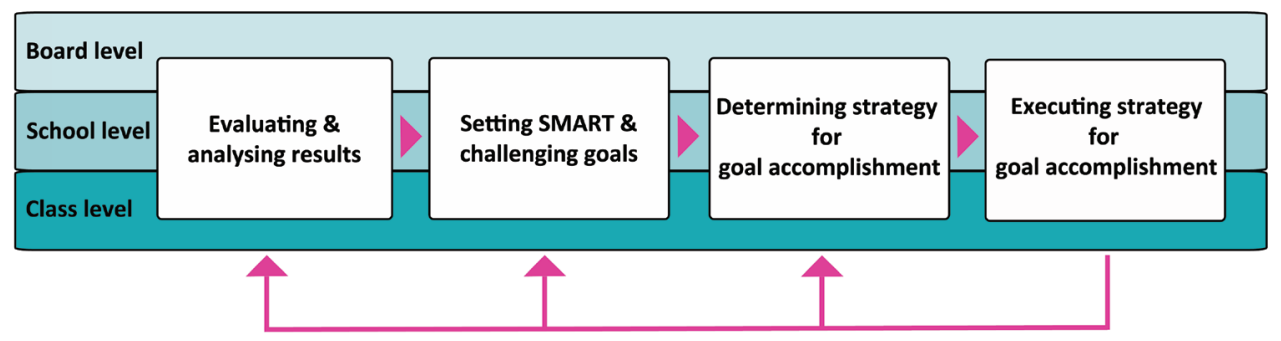

Figure 1. The DBDM cycle

The intervention was provided by DBDM trainers who had been appointed by the University of Twente for this project, and the implementation of the intervention was supervised by the first author who was not directly involved in working with the participating schools.

The first year of the intervention included seven team meetings (each of approximately four hours) aimed at developing DBDM-knowledge and skills. The first four meetings were primarily aimed at DBDM-related knowledge and skills outside the classroom: analyzing and interpreting test score data from the student monitoring system, diagnosing learning needs, setting performance goals, and developing instructional plans. Before the fifth meeting teachers had executed the instructional plans in the classroom, and, based on students' curriculum-based tests, classwork, homework and classroom observations, had adjusted those plans if necessary. By the fifth meeting, the DBDM-cycle had been completed for the first time, and student achievement data were then discussed in a team meeting. During this meeting teachers shared their effective and ineffective classroom practices. 
Meeting six focused on collaboration among team members by preparing for observing each other's lessons, either to learn from the colleague they visited, or to provide him/her with feedback on specific topics. In the last meeting of the school year, the DBDM-cycle was completed for the second time as such results and classroom practices were evaluated again. Furthermore, teachers made an instructional plan for the next school year (and the teacher(s) of that year), and provided information about the class to the new teacher. The second intervention year aimed at deepening, sustaining and broadening DBDM within the school. Since this study examines the changes in teaching quality during the first intervention year, an extensive description of the second intervention year is beyond the scope of this paper.

In the development of the intervention, the features of effective teacher professional development (TPD) were taken into account (Desimone, 2009; Van Veen, Zwart, \& Meirink, 2011). A clear link between newly learned knowledge and skills, together with practice is considered essential (Timperley, 2008; Van Veen et al., 2011). Therefore, when learning how to analyze data, teachers applied what they have learned on data concerning their own students. Also, in the instructional plans teachers learned to develop, they set goals and formulated instructional strategies to achieve these goals for their own classes. During the meetings teachers engaged in active learning, they for example discussed their data analysis results in small groups, or they investigated the alignment of standardized test components and the curriculum. Furthermore, TPD-literature emphasizes that TPD should be consistent with teachers' knowledge and beliefs. Knowledge of and beliefs about DBDM varied between teachers within participating schools. During the intervention, the DBDM-trainer supported schools in developing a shared DBDM-vision.

Since it takes time to learn and change, duration is an important feature of effective professional development in two ways: the number of contact hours and the time span over which the TPD activity is spread (Birman, Desimone, Porter, \& Garet, 2000; Desimone, 2009; Garet, Porter, Desimone, Birman, \& Yoon, 2001). Due to the many other obligations teachers face in their work, they should be provided with sufficient time to master the learning goals (Timperley, 2008; Van Veen et al., 2011). Hence, the DBDM intervention in this study persisted for two years. In the first year there were seven contact meetings, and participants were encouraged to apply and implement in practice what they had learned, for example by carrying out data analyses, developing instructional plans, and, finally adapting their instruction (Timperley, 2008; Van Veen et al., 2011). Finally, collective participation (e.g. as a school team) is positively associated with active participation in professional development activities Garet et al. (2001), Lumpe (2007), and Van Veen, et al. (2011) as well as Timperley (2008) argued that interaction and collaboration between colleagues is important for mastering and implementing an innovation. Therefore, the entire school team participated in the DBDM intervention.

In addition to the seven meetings, teachers were provided with feedback by the DBDM-trainer on both the way they had analyzed and interpreted data as well as on the quality of their instructional plans. Furthermore, teachers were provided with a feedback report about their teaching skills as experienced by their students. In this report the average student scores for each skill were compared to the average across all participating teachers.

To ascertain that the intervention was as consistent as possible across schools and DBDM-trainers, each meeting had a central topic which was the same for every participating school (e.g. analyzing test score data from the student monitoring system, goal setting and developing instructional plans, discussing student progress and the goals for the next period). 
The content of these meetings was fixed for all schools, the same PowerPoint slides were used, and the same exercises were done in all schools. Before each meeting the DBDMtrainers discussed the content for that specific meeting intensively, to assure that each of them would present the information in the same way. Because of variation in school teams' prior knowledge, team members' needs, and the subject chosen by a school, the time a DBDM-trainer spent on a specific topic within a meeting varied somewhat between schools.

Although the content of meetings was very similar across schools, what schools did with the information after the meetings varied and was influenced by several factors such as the characteristics of the school leader, teachers' attitudes towards the intervention, and the time that could be devoted to DBDM.

It was assumed that the features of the intervention as described above would positively influence the knowledge, attitudes and skills of the teachers with regard to DBDM. Thus, it was also expected that teachers would change their instruction, especially with regard to the DBDM teacher skills 'goal-oriented teaching' and 'challenging students' (Desimone, 2009). We will now explain how we measured (changes in) these teaching qualities.

\section{Measuring Teaching Quality by Means of Student Perceptions}

There are several ways to measure teaching quality (Gitomer \& Bell, 2013; Goe, Bell, \& Little, 2008). One way is to use student test scores and determine the added value of a teacher (Guarino, Reckase, \& Wooldridge, 2015). Models used for this purpose are meant to evaluate teachers' contribution to student learning, however, capturing the precise extent to which teacher practices contribute to student learning based on these value-added models is complicated (Goe et al., 2008).

Classroom observations can provide rich information about classroom behaviors and activities (Danielson, 2007; Gitomer \& Bell, 2013; Goe et al., 2008; Pianta et al., 2007; Van de Grift, 2014). However, a valid and reliable measure of teaching quality requires multiple observations and raters (Hill, Charalambous, \& Kraft, 2012). In large-scale studies this is problematic, since teaching quality would have to be measured repeatedly by the same rater or different raters. In the case of one rater, there is a tendency of the rater to score towards what is expected (i.e., rater drift). Therefore, repeated measurements of a rater are correlated and also affected by a rater drift. In the case of different raters, the multiple measurements of a teacher's teaching quality are not correlated, given that raters are randomly assigned to teachers. However, raters may show a lot of variation in measurements of the same teacher, known as inter-rater variability. In that case, the validity of the measurements can be questioned. Raters might interpret measurement procedures differently leading to an inconsistent scoring of a teacher's teaching quality. Besides the practical problems, when using many raters scoring different teachers, a complex correlation structure will be obtained, where measurements are cross-classified within raters and teachers, who are not nested within each other (Hallgren, 2012). Typically for large-scale studies, many raters will be needed and this causes problems with inter-rater reliability and validity. It can also lead to complex cross-classified data structures, which depend on the assignment of raters to teachers. Thus, classroom observations in large-scale studies are not only time-consuming and expensive but it is also hard to obtain valid and reliable measures.

Therefore, in the current study the perceptions of students were used to measure teachers' teaching skills. In contrast with classroom observations, the experiences of students with the behavior of a teacher are based on a large number of lessons (Den Brok, Bergen, Stahl, \& Brekelmans, 2004). Students have encountered many different situations 
with their teacher which may help construct a valid picture of a teacher quality (Goe et al., 2008). Furthermore, where value-added models provide us with information about how much students in the end have learned, student ratings can inform us on the nature of the classroom processes that have contributed to the student outcomes. Such information includes information about teachers' instructional strengths and weaknesses and as such provides a basis for professional development interventions that are in line with teachers' needs (Balch, 2012). Additionally, when students are raters of their teacher, the data analysis becomes less complex, since students are nested within teachers. This leads to a simple hierarchical structure, where student observations are nested within students, who are nested within teachers.

Student ratings are frequently used in higher education as a measure of teaching quality (Spooren, Brockx, \& Mortelmans, 2013) but only rarely in elementary education (Kyriakides, 2005). Possibly, the use of student ratings in elementary school has been avoided, because these students are considered too young to give an accurate judgement of their teachers. However, it looks like this view is gradually changing. De Jong and Westerhof (2001), Peterson, Wahlquist, and Bone (2000), and Kyriakides (2005) for example argue that student perceptions can be used to evaluate elementary school teachers. Recent studies (Balch, 2012; Fauth et al., 2014; Ferguson, 2012; Gaertner, 2014) provided evidence that student perceptions can also be valid in elementary school contexts.

The Tripod student survey, developed by Ferguson (2012), is an example of an instrument designed to measure students' perceptions on seven teaching quality dimensions. The survey was used in the Measurement of Effective Teaching (MET) project (Bill \& Melinda Gates Foundation, 2010) and results showed that elementary school students' ratings of their teachers were positively related with student outcomes (Bill \& Melinda Gates Foundation, 2012).

These results are promising for the use of student ratings in elementary education. However, very little is currently known about changes in student perceptions during a professional development intervention. Few studies measured the development of teacher behavior during an intervention. Ertesvåg (2009) did study the effects of a 'Respect program' (aimed at preventing and reducing problem behavior) on students' perceptions of teachers' emotional support, academic support and monitoring, and she found changes over a period of five years. This is one of the very few studies into the changes in teacher quality during an intervention as measured by means of student perceptions. The current study aims to address the gap in our knowledge by studying the changes in teaching quality during a DBDM intervention by means of student perceptions.

\section{Modelling Changes in DBDM Teaching Skills}

Five teaching skills were examined by means of student perceptions: creating a safe learning climate, classroom management, providing clear instruction, challenging students and goaloriented teaching.

It was expected that student ratings of 'challenging students' and 'goal-oriented teaching' would improve for all teachers after following the DBDM intervention for one year. As no explicit attention was paid to improving the basic teaching skills in the intervention, no changes were expected with regard to 'creating a safe climate', 'classroom management', and 'providing clear instruction'. However, these scales were included to check for possible (negative or positive) side-effects.

Background variables at both the student and the teacher level may explain 
differences in both teachers' pre- and posttest scores. At the student level, grade and gender were taken into account. At the teacher level, teacher characteristics such as gender and years of experience were included. Furthermore, since data were collected over the period of two school years, it was taken into account whether or not teachers changed grades (meaning that the curriculum they taught changed). Finally, whether teachers taught in a multi-grade classroom or a monograde classroom was also included in the model. Executing DBDM-skills in a multi-grade group, might be extra challenging as teachers in these classes have to divide their attention between two or more grades (Little, 2001, 2006; Veenman, 1996). In Figure 3 the conceptual model for 'goal-oriented teaching' is given, which is exemplary for the other four skills studied.

The design of the study. In this pre-posttest design, teachers were rated by their students before the start of the intervention (the control phase) and one year later during the intervention period (the treatment phase). All teachers measured at the pretest were assigned to the treatment. It was not possible to assign teachers to only serve as controls.

This study design has several advantages. Attention is specifically focused on each teacher. It will support statistical inferences about each teacher's performance. The data collected under the design makes it possible to describe in detail the process to investigate change in teachers' performances over time. Furthermore, the data provide information for evidence-based practice. However, the statistical data analysis is more complex, since repeated measurements are correlated over time and correlated within teachers. To make correct statistical inferences, the research design needs to be clearly specified. Furthermore, a statistical model is required which recognizes the complexity of the observed data.

Assume, at the baseline and treatment phase, the order in which the measurements were obtained, was taken into account. Then, repeated measurements were obtained in both phases. Furthermore, without conditioning on teachers' 'goal-oriented teaching', correlated measurements were observed before and during the intervention. This study design relates to the single-subject design (SSD), or single-case design (SCD), where measurements are repeatedly taken over time before and during an intervention (Shadish, Kyse, \& Rindskopf, 2013; Shadish \& Sullivan, 2011)examples are given of how to estimate these models when the single-case designs have features that have not been considered by past authors. These include the use of polynomial coefficients to model nonlinear change, the modeling of counts (Poisson distributed. This study design is used to estimate intra-individual effects, where each teacher also serves as his/her own control. The well-known issues associated with the SSD (e.g., sample size, effect size, serial dependence) can be avoided by means of a multilevel analysis of multiple SSDs concerning all teachers (e.g. Gage \& Lewis, 2012). Since multiple teachers were measured repeatedly, a multilevel modeling approach can be used to estimate an intervention effects across teachers (e.g. Shadish et al., 2013; Van Den Noortgate \& Onghena, 2003).

Although multiple student scores for each teacher were collected in both phases, the measurements were assumed to be independent, given the level of teaching quality, since they were retrieved from different students. Furthermore, the order in which multiple measurements of each teacher were retrieved was assumed to be independent of the scores. Given these assumptions, the serial correlation between (aggregate) measurements over time was only apparent at the level of teachers. Statistical models for data from SSDs were mainly focused on modeling the trend at the lowest level (e.g. Shadish, 2014; Sullivan, Shadish, \& Steiner, 2015). In this study, the correlation between teacher's measurements is modeled 
at a (higher) level of teachers. The presented model for the pre-posttest data extends the hierarchical linear modeling approach of SSD-studies, as students' ratings of their teachers define a separate level of analysis in the model. The performances of teachers are related to each other to define general effects of the DBDM intervention on teaching quality. The typical serial correlation between pre- and posttest scores of teachers are modeled through random teacher effects such that the correlation will not bias the residual errors, parameter estimates and standard errors. By pooling the information from all teachers, reliable and accurate effects of explanatory variables on components of teaching quality can be obtained.

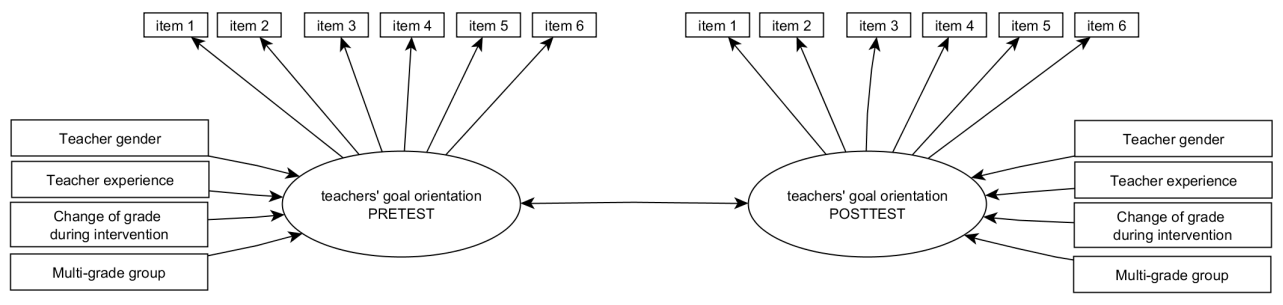
$-\frac{\text { between }}{\text { within }}$
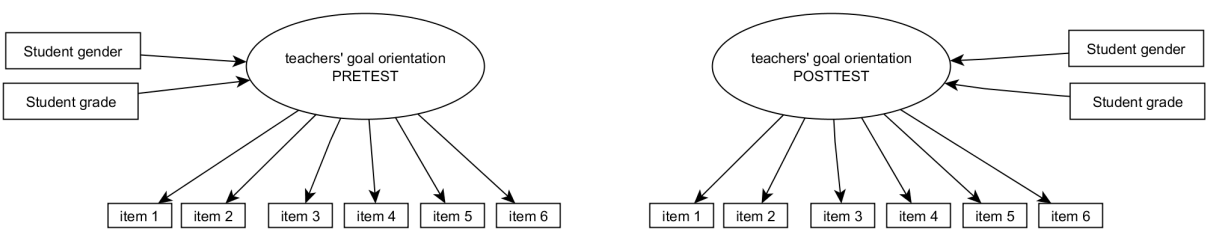

Figure 2. Schematic display of the two-level pre-posttest design concerning teachers' goalorientation

Pre-post multivariate multilevel IRT model. As depicted in Figure 2, the latent variable 'goal-oriented teaching' was measured at the student level with the aim to establish the quality on the teacher level. This nested data structure requires multilevel analysis (Lam, Ruzek, Schenke, Conley, \& Karabenick, 2015). Teachers were graded by students at the start of the first intervention year (pre) and at the start of the second intervention year (post). Since most teachers taught a new class of students in the second intervention year, the same questionnaire was filled out by different students. As a consequence, the number of students grading a teacher varied over the two measurement points- where a teacher may have been graded by twenty students at the pretest, at the posttest twenty-five other students may have filled out the questionnaire about this teacher.

The student latent variables at the pre- and posttest were measured using the graded item response model (Samejima, 1997). At the pretest, the thresholds and discrimination parameters for item $\mathrm{k}\left(k=1, \ldots, K^{\text {pre }}\right)$ are denoted as $\lambda_{k c}^{\text {pre }}$ and $\alpha_{k}^{\text {pre }}$, respectively. The perception of student i about teacher $\mathrm{j}$ at the pre stage is denoted by $\theta_{i j}^{\text {pre }}$. The superscript post is used to refer to the post measurements. The pre- and post-graded item response models were used to measure the perception of student $i$ and / of teacher $j$, respectively, and are given by 


$$
\begin{aligned}
& P\left(Y_{i j k}^{\text {pre }}=c\right)=\Phi\left(\lambda_{k c}^{\text {pre }}-a_{k}^{\text {pre }} \theta_{i j}^{\text {pre }}\right)-\Phi\left(\lambda_{k(c-1)}^{\text {pre }}-a_{k}^{\text {pre }} \theta_{i j}^{\text {pre }}\right) \\
& P\left(Y_{l j k}^{\text {post }}=c\right)=\Phi\left(\lambda_{k c}^{\text {post }}-a_{k}^{\text {post }} \theta_{l j}^{\text {post }}\right)-\Phi\left(\lambda_{k(c-1)}^{\text {post }}-a_{k}^{\text {post }} \theta_{l j}^{\text {post }}\right)
\end{aligned}
$$

To ensure that the pre- and post-measurement of students' perceptions were on the same scale, three anchor items were selected. These anchor items were assumed to function equally over measurement occasions. Student perceptions at the pre- and post-event were used to measure teachers' goal-orientation. As depicted in Figure 2, pre- and postmeasures are assumed to be correlated at the teacher-level, but not at the student-level as different students completed the questionnaire at both time points. Subsequently, a multivariate multilevel model can be defined for the student perceptions, while controlling for the correlation between each teacher's pre- and post-measurements. For the pre- and posttest measurements, student level-1 models can be defined to model the variability in perceptions given teachers' goal-orientation, and student and teacher explanatory variables $X=\left(X^{\text {pre }}, X^{\text {post }}\right)$, given by

$$
\begin{aligned}
& \theta_{i j}^{\text {pre }}=\beta_{0 j}^{\text {pre }}+\mathbf{X}_{i j}^{\text {pre }} \boldsymbol{\beta}^{\text {pre }}+e_{i j}^{\text {pre }} \\
& \theta_{l j}^{\text {post }}=\beta_{0 j}^{\text {post }}+\mathbf{X}_{l j}^{\text {post }} \boldsymbol{\beta}^{\text {post }}+e_{l j}^{\text {post }}
\end{aligned}
$$

where the error components are assumed to be independently normally distributed with an occasion-specific variance, denoted as $\tau_{\text {pre }}^{2}$ and $\tau_{\text {post }}^{2}$. The random teacher effects are assumed to be multivariate normally distributed to address the correlation between each teacher's measurements. This level-2 part is given by

$$
\left(\begin{array}{l}
\beta_{0 j}^{\text {pre }} \\
\beta_{0 j}^{\text {post }}
\end{array}\right) \sim N\left(\left(\begin{array}{c}
\gamma_{0}^{\text {pre }} \\
\gamma_{0}^{\text {post }}
\end{array}\right),\left(\begin{array}{cc}
\sigma_{\text {pre }}^{2} & \sigma_{\beta} \\
\sigma_{\beta} & \sigma_{\text {post }}^{2}
\end{array}\right)\right)
$$

where $\gamma_{0}^{\text {pre }}$ and $\gamma_{0}^{\text {post }}$ are the expected population-average perceptions at the pretest and posttest, respectively. The covariance matrix represents the variability in a teacher's goalorientation at the pre- and post-measurement, and $\sigma_{\beta}$ represents the covariance between a teacher's pre- and post-measurement.

The multiple student measurements were used to measure teaching skills of teachers at the pretest and posttest, according to Equation 2. Without conditioning on explanatory variables, the reliability, $\lambda_{j}$, can be measured of each teacher's measurement at the pretest and posttest, denoted as $\beta_{0 j}^{\text {pre }}, \beta_{0 j}^{\text {post }}$, respectively. According to classical test theory, the reliability can be defined as the ratio of the variance of the latent variable variance relative to the total variance of the average student perceptions, denoted as $\bar{\theta}_{j}$ (Raudenbush \& Bryk, 2002, p. 46). The reliability terms for the pretest and posttest measurement of teacher $j$ are given by

$$
\lambda_{j}^{\text {pre }}=\operatorname{var}\left(\beta_{0 j}^{\text {pre }}\right) / \operatorname{var}\left(\bar{\theta}_{j}^{\text {pre }}\right)=\frac{\sigma_{p r e}^{2}}{\sigma_{p r e}^{2}+\tau_{p r e}^{2} / n_{j}}
$$

and, 


$$
\lambda_{j}^{\text {post }}=\operatorname{var}\left(\beta_{0 j}^{\text {post }}\right) / \operatorname{var}\left(\bar{\theta}_{j}^{\text {post }}\right)=\frac{\sigma_{\text {post }}^{2}}{\sigma_{\text {post }}^{2}+\tau_{\text {post }}^{2} / n_{j}},
$$

respectively, where $\tau_{\text {pre }}^{2}$ and $\tau_{\text {post }}^{2}$ are the occasion-specific error variances at the level of students. Furthermore, let $n_{j}$ denote the number of students grading teacher $j$. The reliability coefficients $\lambda_{j}^{\text {pre }}$ and $\lambda_{j}^{\text {post }}$ are high when the teachers differ substantially in their teaching quality ( $\sigma_{\text {pre }}^{2}, \sigma_{\text {post }}^{2}$ are high) or when the number of students, $n_{j}$, is high.

To identify the mean and variance at both test occasions, the mean and variance of the measured student perceptions at the pretest were fixed at zero and one respectively. This is a common identifying restriction in multiple group IRT modeling (Azevedo, Andrade, \& Fox, 2012; Bock \& Zimowski, 1997; Reise, Widaman, \& Pugh, 1993). Therefore, $\gamma_{0}^{\text {pre }}=0$ and the total variance of $\theta_{i j}^{\text {pre }}$ is restricted to one. However, the restriction on the total variance at the pretest is included in the estimation method, since the multilevel modeling of the premeasurements avoids a restriction on one single variance parameter.

The multivariate two-level model for the latent pre- and post-measurements can be stated as,

$$
\begin{aligned}
& \theta_{i j}^{\text {pre }}=\mathbf{X}_{i j}^{\text {pre }} \boldsymbol{\beta}^{\text {pre }}+u_{0 j}^{\text {pre }}+e_{i j}^{\text {pre }} \\
& \theta_{l j}^{\text {post }}=\gamma_{0}^{\text {post }}+\mathbf{X}_{l j}^{\text {post }} \boldsymbol{\beta}^{\text {post }}+u_{0 j}^{\text {post }}+e_{l j}^{\text {post }}
\end{aligned}
$$

where the teacher-level error components $\left(u_{0 j}^{\text {pre }}, u_{0 j}^{\text {post }}\right)$ are multivariate normally distributed and the student-level error components are independently distributed, since different students over test occasions are nested within a common teacher $j$. The parameter $\gamma_{0}^{\text {post }}$ represents the average pre-post effect, representing the average score differences between the pre- and post-measurements given the explanatory variables. Note that the student and teacher latent variables are on a common scale due to the anchor items and the correlation between teacher measurements.

The multivariate multilevel analysis of pre- and posttest scores has the advantage that independent explanatory variables at the student and teacher level (e.g., student and teacher gender, teacher experience) can be added to the model at both time points. This makes it possible to compare the effects of the explanatory variables from pre- to posttest. Furthermore, in the multivariate analysis the measurement errors of both measurements can be taken into account and can be defined independently from each other. Differential measurement errors are allowed at the level of students and teachers. It is also possible to include time-invariant and time-variant explanatory variables, which both can differ in their effects on the pre- and post-measurements.

In the traditional change score method (e.g. Allison, 1990), the difference score $\beta_{0 j}^{\text {post }}-\beta_{0 j}^{\text {pre }}$ would be considered to be a dependent variable. However, the reliability of this change score is complex, since the teacher measurements are based on different student constructs, which are not likely to be equally reliable. When ignoring the (differential) measurement error of student and/or teacher construct scores, estimates of pre-post effects might be biased. Furthermore, when effects of explanatory variables are not invariant from the pretest to posttest, they need to be included to account for differences between the two occasions. When the change score method is utilized, it is only possible to address effects of pretest-posttest differences in explanatory variables, but not of the actual effects at the occasions. This complicates the interpretation of the effects of explanatory variables. Time- 
invariant explanatory variables will drop out of the equation, although their effects might be different at the pre- and posttest.

In the so-called regressor variable approach, the pre-measurement is used as a control variable, which would mean that $\beta_{0 j}^{\text {post }}$ is regressed on $\beta_{0 j}^{\text {pre }}$ and explanatory variables. In this case, the measurement error associated with the pre-measurement can produce biased estimates of the pre-post effect. Furthermore, in correspondence with the change score method, the inclusion of explanatory variables is restricted to changes in the variables measured at both occasions. The regressor variable approach is implied by the multivariate model in Equation 3. When considering the conditional distribution of $\beta_{0 j}^{\text {post }}$ given $\beta_{0 j}^{\text {pre }}$, it follows that

$$
\beta_{0 j}^{\text {post }} \mid \beta_{0 j}^{\text {pre }} \sim N\left(\gamma_{0}^{\text {post }}+\frac{\sigma_{\beta}}{\sigma_{\text {pre }}^{2}}\left(\beta_{0 j}^{\text {pre }}-\gamma_{0}^{\text {pre }}\right), \sigma_{\text {post }}^{2}-\frac{\sigma_{\beta}^{2}}{\sigma_{\text {pre }}^{2}}\right)
$$

which shows that the covariance between measurements is the unscaled regression effect of $\beta_{0 j}^{\text {post }}$ regressed on $\beta_{0 j}^{\text {pre }}$. However, in this case, the measurement error is included in the predictor, and the mean term is controlled for occasion differences using occasion-specific explanatory variables.

The joint model for measuring constructs using the graded IRT model, and the multivariate multilevel model to analyze the pre-post measurements of students and teachers, will be referred to as the pre-post multivariate multilevel IRT model. This extends the multilevel IRT model of Fox and Glas (2001) and Fox (2010). In the multilevel IRT model, student constructs are measured, which are nested in higher level units (e.g., teachers, schools). In this generalization, the higher-level measurements are allowed to correlate and to be measured at different occasions.

\section{Methodology}

Data for this study were gathered from 209 grade 2 to 6 teachers in the Netherlands which participated in the DBDM intervention. Data were collected at the start and after one year of DBDM-implementation. In this section, the sample and method of data collection are described first, after which a description of how the data were analyzed is presented.

\section{Sample}

As part of the DBDM intervention schools were given the opportunity to administer student questionnaires about the quality of teaching in grades 2 to 6 . Although all team members participated in the DBDM intervention, no student perception data were collected for teachers in Kindergarten and grade 1, since these students were considered to be too young to give a valid and reliable measure. For the current sample, complete cases were selected, where students filled out a questionnaire at the start of the first year, and at the start of the second school year. This resulted in a data sample of 209 teachers from 37 schools. In Table 1 the characteristics of the participating teachers are presented. During both the pretests and the posttests, 22 students per teacher filled out the questionnaire on average. Some teachers were rated by two classes of students, for example because a teacher taught the first part of the week in one class, and the second part of the week in the other class, resulting in approximately 50 students who filled out a questionnaire about this teacher. Similar to the overall Dutch situation, a minority of teachers in this sample was male. Moreover, these male 
Table 1. Teacher characteristics $(N=209)$

\begin{tabular}{lccccc}
\hline & $\mathbf{N}$ & Min & Max & Mean & SD \\
\hline $\begin{array}{l}\text { Average age } \\
\text { Years of experience (at the start of the }\end{array}$ & 207 & 24 & 64 & 43.79 & 12.19 \\
$\begin{array}{l}\text { intervention) } \\
\text { Number of students who filled out the }\end{array}$ & 192 & 1 & 40 & 16.19 & 11.91 \\
$\begin{array}{l}\text { questionnaire at the pretest } \\
\text { Number of students who filled out the }\end{array}$ & 209 & 7 & 53 & 21.98 & 6.00 \\
questionnaire at the posttest & 209 & 6 & 54 & 22.01 & 6.12 \\
Male & & & & & \\
Female & 48 & $(23.0 \%)$ & & & \\
Grade per time point & 160 & $(76.6 \%)$ & & & \\
Grade 2 & & & & & \\
Multigrade group 2/3 & Pre & & & 32 & $(15.3 \%)$ \\
Grade 3 & 34 & $(16.3 \%)$ & & 6 & $(2.9 \%)$ \\
Multigrade group 3/4 & 6 & $(2.9 \%)$ & & 8 & $(14.8 \%)$ \\
Grade 4 & 33 & $(15.8 \%)$ & & 34 & $(16.3 \%)$ \\
Multigrade group 4/5 & 10 & $(4.8 \%)$ & & 7 & $(3.3 \%)$ \\
Grade 5 & 30 & $(14.4 \%)$ & & 37 & $(17.7 \%)$ \\
Multigrade group 5/6 & 5 & $(2.4 \%)$ & & 10 & $(4.8 \%)$ \\
Grade 6 & 37 & $(17.7 \%)$ & & 44 & $(21.1 \%)$ \\
Other & 9 & $(4.3 \%)$ & & & \\
\hline
\end{tabular}

Table 2. Student characteristics for pretest $(n=4,593)$ and posttest $(n=4,598)$

\begin{tabular}{clcccc}
\hline & & N & Pretest & \multicolumn{2}{c}{ Posttest } \\
& & (\%) & N & (\%) \\
\hline Gender & Boys & 2218 & $(48.3 \%)$ & 2264 & $(49.2 \%)$ \\
& Girls & 2285 & $(49.7 \%)$ & 2259 & $(49.1 \%)$ \\
\multirow{6}{*}{ Grade } & Missing & 90 & $(2.0 \%)$ & 75 & $(1.6 \%)$ \\
& 2 & 743 & $(16.2 \%)$ & 743 & $(16.2 \%)$ \\
& 3 & 934 & $(20.3 \%)$ & 934 & $(20.3 \%)$ \\
& 4 & 871 & $(19.0 \%)$ & 871 & $(19.0 \%)$ \\
& 5 & 1048 & $(22.8 \%)$ & 1048 & $(22.8 \%)$ \\
& 6 & 994 & $(21.6 \%)$ & 994 & $(21.6 \%)$ \\
& Missing & 3 & $(0.1 \%)$ & 3 & $(0.1 \%)$ \\
\hline
\end{tabular}


teachers mainly taught in the higher grades. At the pretest, 32 out of 48 males taught in grades 5 and 6 , and at the posttest this number was even higher (36).

Generally, students had the same teacher (or the same teachers in the case of a job share) throughout the school year. With the start of a new school year teachers usually work with a new group of students. In our sample, 112 teachers stayed in the same grade, and thus worked with another group of students. The other 97 teachers started to teach in a new grade, of which 41 teachers moved to a lower grade (e.g. grade 5 in the first year, grade 3 in the second year), 53 teachers moved to a higher grade, and three teachers taught multiple grades - but not as one multigrade group - in the first year, and one grade in the second year (e.g. grade 3 and grade 5 in the first year and grade 5 in the second year). In sum, the majority of the students who filled out the questionnaire about a specific teacher at the pretest were not the same students as those who filled out the questionnaire about this same teacher at the posttest. Furthermore, since the students filled out the questionnaire anonymously, it was not possible to follow students from pretest to posttest. Student characteristics are presented in Table 2 .

\section{Instrument}

Based on the five teaching skills, creating safe climate, classroom management, providing clear instruction, challenging students and goal-oriented teaching, a student questionnaire was developed. The Tripod Survey of Ferguson (2012) served as the initial source of inspiration. Questions were translated into Dutch, and as a result of a pilot study with 59 teachers and the recommendations of an expert group of elementary education teachers, some questionnaire items were excluded, others were further adjusted to match the Dutch context, and various new items were included in the questionnaire. This resulted in a questionnaire containing 36 items divided over the five subscales (ranging from five to ten items per scale). All questions were scored on a five-point Likert-scale, ranging from 'no, never' to 'yes, always'. A translation of the questionnaire can be found in the Table A.1 in Appendix C.

For each teaching skill, on both pretest and posttest, the reliability was calculated using equation 4 and 5 . The results are presented in Table 3. For each teaching skill, the average reliability (across students and teachers) is above .80. This means that the used scales, in combination with students as raters, support reliable measurements of teaching skills. Furthermore, substantial differences in teaching skills across teachers were measured in a reliable way. The used scales made it possible to discriminate well between teacher's performances. The reliability of the measurements of teaching skills differ, since each teacher was rated by different number of students at the pretest and at the posttest. At the pretest, this ranged from 7 to 53 students $(M=22)$ and at the posttest this ranged from 6 to $54(M=$ 22).

Table 3. Estimates of reliability coefficients $\lambda_{i}^{\text {pre }}$ and $\lambda_{i}^{\text {post }}$ for all five teaching skills ( $\left.N=209\right)$

\begin{tabular}{|c|c|c|c|c|c|c|c|c|c|c|}
\hline & \multicolumn{2}{|c|}{$\frac{\text { Creating a safe }}{\text { climate }}$} & \multicolumn{2}{|c|}{$\begin{array}{c}\text { Classroom } \\
\text { management }\end{array}$} & \multicolumn{2}{|c|}{$\frac{\text { Providing clear }}{\text { instruction }}$} & \multicolumn{2}{|c|}{$\begin{array}{l}\text { Challenging } \\
\text { students }\end{array}$} & \multicolumn{2}{|c|}{$\frac{\text { Goal-oriented }}{\text { teaching }}$} \\
\hline & $\lambda_{j}^{\text {pre }}$ & $\lambda_{j}^{\text {post }}$ & $\lambda_{j}^{\text {pre }}$ & $\lambda_{j}^{\text {post }}$ & $\lambda_{j}^{\text {pre }}$ & $\lambda_{j}^{\text {post }}$ & $\lambda_{j}^{\text {pre }}$ & $\lambda_{j}^{\text {post }}$ & $\lambda_{j}^{\text {pre }}$ & $\lambda_{j}^{\text {post }}$ \\
\hline Mean & .86 & .85 & .91 & .89 & .83 & .82 & .85 & .84 & .87 & .91 \\
\hline Min. & .67 & .62 & .77 & .71 & .62 & .58 & .66 & .61 & .70 & .74 \\
\hline Max. & .94 & .94 & .96 & .96 & .92 & .92 & .94 & .93 & .95 & .96 \\
\hline
\end{tabular}




\section{Model Estimation}

The joint estimation of all pre-post multivariate multilevel IRT model parameters as in Equation 1 and Equation 6 could not be carried out with existing statistical software. Therefore, a Markov chain Monte Carlo (MCMC) algorithm was implemented in R (R Core Team, 2014) by extending the MCMC methods for multilevel IRT models (e.g. Fox \& Glas, 2001; Fox, 2010; Stone \& Zhu, 2015). The identification of the model was included in the MCMC algorithm. Complete missing cases, where teachers were only assessed at the pretest, or at the posttest were not allowed. Students with incomplete response patterns were allowed, and missing values at the level of responses were assumed to be missing at random (MAR). Therefore, during each iteration of the MCMC algorithm, a value was generated for each missing value using the model and the current information about the model parameters. Across MCMC iterations, this imputation method generated the most probable observations for the missing responses given the posterior distribution of the model parameters.

The estimation method was evaluated through a simulation study. Fifty data sets were sampled according to the model, where the true parameter values are given in Table 4. Data for 1000 students nested in 100 teachers were sampled, according to a ten-item pretest and a twelve-item posttest with three response categories. The MCMC algorithm required a burn-in period of 1000 iterations, and subsequently 5000 iterations were used to estimate all model parameters. The 5000 iterations delivered sampled parameter values from their posterior distributions. In this Bayesian estimation procedure, all inferences are based on the information from the posterior distributions. Therefore, the estimated mean and standard deviation of the sampled values for each parameter were used as an estimate of the parameter and its standard deviation, respectively.

It can be seen in Table 4, that the true parameter values were recovered. Each reported parameter estimate is the average of the estimates from the fifty replicated data sets. It was concluded that the designed MCMC algorithm correctly produced estimates for the pre-post multivariate multilevel IRT model parameters.

Table 4. Results simulation study for the parameter recovery of the pre-post multivariate multilevel model

\begin{tabular}{|c|c|c|c|c|c|c|}
\hline & \multicolumn{2}{|c|}{$\underline{\text { True values }}$} & \multicolumn{4}{|c|}{$\frac{\text { Estimated values }}{\text { (over } 50 \text { replications) }}$} \\
\hline & \multirow[t]{2}{*}{ Pretest } & \multirow[t]{2}{*}{ Posttest } & \multicolumn{2}{|c|}{ Pretest } & \multicolumn{2}{|c|}{ Posttest } \\
\hline & & & M & (SD) & M & (SD) \\
\hline \multicolumn{7}{|l|}{ Fixed effects } \\
\hline Intercept $\gamma_{0}^{\text {post }}$ & .00 & -.19 & .00 & (.08) & -.19 & $(.07)$ \\
\hline Student predictor & .40 & .92 & .38 & $(.07)$ & .95 & $(.00)$ \\
\hline \multicolumn{7}{|l|}{$\underline{\text { Random effects }}$} \\
\hline Level-2 variance $\sigma_{p r e}^{2}, \sigma_{p o s t}^{2}$ & 1.00 & 1.158 & 1.01 & $(.05)$ & 1.18 & (.12) \\
\hline Level-1 variance $\tau_{\text {pre }}^{2}, \tau_{\text {post }}^{2}$ & .50 & .300 & .51 & (.09) & .32 & $(.07)$ \\
\hline \multirow{2}{*}{$\underline{\text { Covariance pre-post }} \sigma_{\beta}$} & .15 & & .15 & $(.06)$ & & \\
\hline & .39 & & .37 & $(.13)$ & & \\
\hline
\end{tabular}




\section{Data Analysis Plan}

The analyses started with an empty model, which did not contain any explanatory variables. This empty model provided the item parameters difficulty and discrimination, as well as the unconditional treatment effect. Next, the explanatory variables as proposed in the model shown in Figure 2 were included stepwise.

In order to make inferences about the effects of the explanatory variables on the pretest as well as the posttest, highest posterior density (HPD) intervals were calculated. The Bayesian 95\% confidence intervals can be interpreted as the interval which includes the model parameter with a 95\% posterior probability. The 95\% HPD interval is the Bayesian 95\% confidence interval for which each point within the interval has a higher posterior density than any point outside of the interval. For a HPD interval, the most likely parameter values are always included. If the point zero is not included in the 95\% HPD interval, then it can be concluded that the parameter is significantly different from zero with a 95\% posterior probability (e.g. Fox, 2010, p. 59).

Explanatory variables which had no significant effect on both pretest and posttest factor scores were excluded from the model. The empty model was used to investigate the amount of level-1 variance between students, and the level-2 variance between teachers. Let $\tau_{\text {pre }}^{2}$ and $\tau_{\text {post }}^{2}$ denote the student-level variance components at the pretest and the posttest. Then, the intra-class correlation coefficient at the pre- and post-measurement can be defined as

$$
\rho_{\text {pre }}=\frac{\sigma_{p r e}^{2}}{\sigma_{p r e}^{2}+\tau_{p r e}^{2}} \text {, and } \rho_{\text {post }}=\frac{\sigma_{p o s t}^{2}}{\sigma_{p o s t}^{2}+\tau_{p o s t}^{2}} \text {. }
$$

Subsequently, the reduction in level-1 and level-2 variance can be computed at the pretest and posttest. Following (Snijders \& Bosker, 1999, pp. 102-103), the proportional reduction in variances at the pretest are given by

$$
R_{p r e, 1}^{2}=1-\frac{\tau_{p r e}^{2}\left(\mathbf{X}_{p r e}\right)}{\tau_{p r e}^{2}} \text {, and } R_{p r e, 2}^{2}=1-\frac{\sigma_{p r e}^{2}\left(\mathbf{X}_{p r e}\right)}{\sigma_{p r e}^{2}}
$$

where, for example, the $\tau_{\text {pre }}^{2}\left(\mathbf{X}_{\text {pre }}\right)$ denotes the level-1 variance when including the predictor variables $\mathbf{X}_{\text {pre }}$. The MCMC algorithm for parameter estimation can also be used to obtain sampled values of functions of parameters from their posterior distributions, such as the intra-class correlation and the $R_{1}^{2}$ and $R_{2}^{2}$ (i.e., the proportional reduction in level-1 and level-2 variances). The sampled function values are retrieved from their posterior distributions such that the mean, variance and HPD interval can also be computed for the intra-class correlation and the $R_{1}^{2}$ and $R_{2}^{2}$ at the pretest and posttest.

Finally, through the final model, it was investigated whether the effects of explanatory variables had changed over time. This was done by calculating the mean and HPD of the difference in these effects. The differences between the effects again can be considered to be a function of the model parameters. The sampled effect values at the pretest and posttest can be obtained from their posterior distributions using the MCMC algorithm. Subsequently, the sampled differences can be constructed and used to estimate the mean, variance and HPD interval of the difference in effects of variables at the pretest and posttest. 


\section{Results}

For each scale, the three items with the highest discriminating power were selected as anchor items. The invariance assumption of the chosen anchor items was evaluated. Therefore, other anchors were selected and the parameters of the originally chosen anchors were estimated for the pretest and posttest. If the estimated individual item parameters for the pretest were significantly different from those for the posttest, it was concluded that the invariance assumption did not hold and in that case the item could not serve as an anchor. The largest differences in item parameters were found for the 'safe climate' scale, however, these were still within the 95\% HPD intervals of the item parameters. The parameters of the non-anchor items also do not reveal much variability in item characteristics over test occasions. The estimated item parameters are given in Table A.1 and A.2 of Appendix C.

Analyses were performed separately for the five skills. Since our primary focus was on goal-orientation and challenging students, we chose to describe the analysis of the first DBDM-skill 'goal-oriented teaching' extensively in the following section. Next, a brief description of the results for the other four scales is given. Furthermore, the elaborate analysis of the remaining four skills can be found in Appendix $C$.

\section{Goal-Oriented Teaching}

The scale 'goal-oriented teaching' consisted of six items. Based on the empty model, the thresholds $\left(\lambda_{k c}^{\text {pre }}, \lambda_{k c}^{\text {post }}\right)$ and discrimination parameters $\left(\alpha_{k}^{\text {pre }}, \alpha_{k}^{\text {post }}\right)$ of the items were calculated at the pre- and post-measure (these can be found in Appendix C). The discrimination parameters ranged from .51 to 1.04, which showed sufficient discriminating power to rank the students and teachers on a common scale. For all items, the estimated response category boundaries (i.e., the thresholds) are well-spread, as a result the perceptions of students at the lower and higher end of the scale can be measured accurately. The thresholds also reveal that it appeared to be more common for teachers to tell students what they are going to learn (item 5.1) rather than explaining what was covered in the previous lesson (item 5.5). The estimated response category bounds of the items showed a more or less equal range in difficulties over tests. An exception was item 5.3, where it appeared to be more frequent that teachers marked students' work to improve students' understanding at the pretest compared to the posttest. In Table 5 the results of the empty model are presented.

The nested structure of the data, where responses are nested within students, and students nested within teachers, is captured by two random effects per measurement occasion: the random effect at the teacher level $\left(\beta_{0 j}^{\text {pre }}, \beta_{0 j}^{\text {post }}\right)$, and at the student level ( $\theta_{i j}^{\text {pre }}$, $\left.\theta_{l j}^{\text {post }}\right)$. The random effect variances indicate that the largest part of the variance is explained at the student level. The student-level random effect identifies the clustering of responses within subjects. The intra-class correlation estimates (to explain the proportion of variance due to the clustering of students in teachers) show that at the pretest $25 \%$ of the variance is explained by the teacher clustering. At the posttest, this percentage significantly increased to $32 \%$ (mean difference $=.07$, HPD: $[.00, .14]$ ), meaning that the influence of the teacher-level increases overall. The decrease in level-1 variance indicates that students at the posttest agree more with each other about teachers' goal-orientation compared to the pretest.

The intercept is the overall teacher score on the 'goal-oriented teaching' scale. The mean of the latent scale is identified by restricting the mean of the factor scores to zero on the pretest. Due to this restriction on the pretest scale scores, the intercept is approximately equal to zero, since it identifies the mean of the reference scale. The estimated intercept 
Table 5. Empty model - goal-oriented teaching

\begin{tabular}{|c|c|c|c|c|c|c|}
\hline & \multicolumn{3}{|c|}{ Pretest } & \multicolumn{3}{|c|}{ Posttest } \\
\hline & Estimate & SD & HPD & Estimate & SD & HPD \\
\hline \multicolumn{7}{|l|}{ Fixed effects- level 2} \\
\hline Intercept $\gamma_{0}^{\text {post }}$ & .04 & .04 & {$[-.04, .11]$} & .29 & .05 & {$[.20, .39]$} \\
\hline \multicolumn{7}{|l|}{ Random effects } \\
\hline Level-2 variance $\sigma_{p r e}^{2}, \sigma_{\text {post }}^{2}$ & .26 & .03 & {$[.20, .32]$} & .34 & .04 & {$[.26, .42]$} \\
\hline Level-1 variance $\tau_{p r e}^{2}, \tau_{\text {post }}^{2}$ & .77 & .02 & {$[.73, .81]$} & .71 & .03 & {$[.65, .76]$} \\
\hline$\rho_{\text {pre }}, \rho_{\text {post }}$ & .25 & .02 & {$[.20, .30]$} & .32 & .03 & {$[.28, .38]$} \\
\hline Covariance pre-post $\sigma_{\beta}$ & .18 & .03 & {$[.13, .23]$} & & & \\
\hline Correlation pre-post & .61 & .08 & {$[.45, .76]$} & & & \\
\hline
\end{tabular}

at the posttest of $\gamma_{0}^{\text {post }}=.29$ implies that, in general, teachers' goal-orientation increased compared to the pretest, without controlling for background differences. On average, across teachers and students, the population average score is significantly higher on the posttest compared to the pretest. Note that this difference is measured on a common scale through the use of anchor items.

The factor scores of teachers on the pretest are plotted against those on the posttest in Figure 3. Teachers who scored below the straight grey line did not improve their score on the posttest. Apparently, teachers tended to score higher on the posttest compared to the pretest. The dotted fitted line has a slope of .70, which is the estimated regression effect of teachers' pretest scores on posttest scores. The regression effect is calculated by

according to Equation 7.

$$
\text { slope }=\rho * \frac{\sigma_{\text {post }}}{\sigma_{\text {pre }}}
$$

For repeated measurements, a correlation of .61 between pretest and posttest scores is relatively low. This indicates that, on average, teachers' goal-orientation changed in the period from pre- to posttesting. Although the degree of correlation shows that the posttest scores differ from the pretest scores, measurement error and other error components can also diminish the strength of the relation.

Next, we ran multiple models including the explanatory variables stepwise. Explanatory variables that had no significant effect on both pre- and posttest factor scores were excluded from the subsequent models. Whether a teacher changed grades had no effect on the teacher's goal-orientation, and neither did the gender of the teacher influence the factor score $\left(\theta_{i j}^{\text {pre }}, \theta_{l j}^{\text {post }}\right)$. In the final model, shown in Table 6, the explanatory factor variables 'grade', 'student gender', 'teacher experience', and whether the teacher taught a 'multi-grade group' or not were included.

To test how much the model explains, the explained proportion of variance was calculated. The level-1 explained proportion of variance $R_{1}^{2}$ was .01 for the posttest and .07 for the pretest suggesting that the included variables explained little variance within 


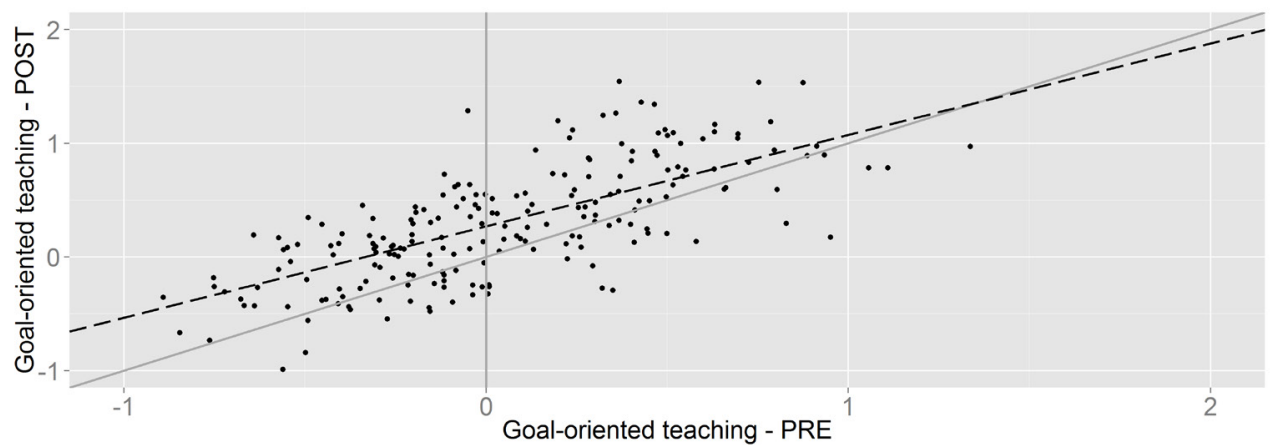

Figure 3. Teachers' factor scores on pretest plotted against scores on posttest. The dotted line represents the linear regression of estimated pretest scores on posttest scores. The grey line represents the line of a perfect correlation between pre-scores and post-scores, and the vertical grey line represents the population-average pretest score of zero

Table 6. Final model-goal-oriented teaching

\begin{tabular}{|c|c|c|c|c|c|c|}
\hline & \multicolumn{3}{|c|}{ Pretest } & \multicolumn{3}{|c|}{ Posttest } \\
\hline & Estimate & SD & HPD & Estimate & SD & HPD \\
\hline \multicolumn{7}{|l|}{ Fixed effects- level 2} \\
\hline Intercept & -.15 & .08 & {$[-.30, .00]$} & .20 & .08 & {$[.03, .34]$} \\
\hline (grand mean centered) & -.01 & .00 & {$[-.02, .00]$} & -.01 & .00 & {$[-.01, .00]$} \\
\hline Multigrade group & -.24 & .09 & {$[-.41,-.08]$} & -.08 & .09 & {$[-.28, .09]$} \\
\hline \multicolumn{7}{|l|}{ Non-multigrade $=$ ref } \\
\hline \multicolumn{7}{|l|}{ Fixed effects - level 1} \\
\hline Grade 2 & .54 & .11 & {$[.34, .76]$} & .61 & .11 & {$[.38, .85]$} \\
\hline Grade 3 & .17 & .10 & {$[-.02, .36]$} & .07 & .11 & {$[-.13, .29]$} \\
\hline Grade 4 & .23 & .09 & {$[.05, .39]$} & -.01 & .10 & {$[-.21, .16]$} \\
\hline Grade 5 & .11 & .09 & {$[-.07, .27]$} & -.01 & .09 & {$[-.19, .15]$} \\
\hline Grade $6=$ ref & & & & & & \\
\hline Girl & .07 & .03 & {$[.01, .13]$} & -.03 & .03 & {$[-.09, .03]$} \\
\hline Boy $=$ ref & & & & & & \\
\hline \multicolumn{7}{|l|}{$\underline{\text { Random effects }}$} \\
\hline Level-2 variance $\sigma_{\text {pre }}^{2}, \sigma_{\text {post }}^{2}$ & .22 & .03 & {$[.17, .28]$} & .28 & .04 & {$[.21, .35]$} \\
\hline Level-1 variance $\tau^{2}, \tau^{2}$ & .76 & .02 & {$[.73, .80]$} & .67 & .04 & {$[.59, .73]$} \\
\hline & .23 & .02 & {$[.18, .27]$} & .29 & .03 & {$[.25, .35]$} \\
\hline \multicolumn{7}{|l|}{$\rho_{\text {pre }}, \rho_{\text {post }}$} \\
\hline Covariance pre-post $\sigma_{\beta}$ & .15 & .02 & {$[.10, .19]$} & & & \\
\hline$\overline{\text { Correlation pre-post }}$ & .59 & .08 & {$[.44, .75]$} & & & \\
\hline
\end{tabular}


teachers. The level-2 explained proportion of variance $R_{2}^{2}$ was .12 for the pretest and .19 for the posttest.

In this final model, the measurements of teachers' goal orientation was disentangled by the explanatory factor variables 'grade', 'student gender' and teaching a 'multi-grade group'. Examination of the effects of the explanatory variables on the pretest indicated that students in the lowest grade were most positive about their teachers' goal-oriented teaching. Next, the results suggest that girls at the pretest were slightly more positive about goalorientation compared to boys. 'Teacher experience' was included as a continuous variable and was centered around its mean. The negative effect suggests that the older a teacher, the lower his/her goal-orientation was rated by his/her students. Finally, teachers who taught a multi-grade group had a considerably lower score at the pretest compared to teachers teaching one grade.

At the posttest, the intercept, $\left(\gamma_{0}^{\text {post }}\right)$, can be interpreted as the average perception of boys in grade 6 having a teacher of a monograde group with a population average of years of experience. Estimates of the effects of the explanatory factor variables on the posttest suggest that apart from grade 2, the explanatory variables did not reveal any significant differences in teachers' goal-orientation. In turn, based on the 95\% HPD interval, the decrease of the effects was only statistically meaningful for 'student gender' (mean difference $=-.10$, HPD: $[-.19,-.02]$ ), and grade 4 compared to grade 6 (mean difference $=.24$, HPD: $[-.48$, -.01]). These findings provide evidence that, compared to the pretest, students were more in agreement about their teachers' goal-orientation, where other background variables did not explain differences between teachers anymore. This finding was supported by the increased teacher-level variance at the posttest compared to the pretest and, as a result, an increase of the intra-class correlation.

Differences in student perceptions of their teachers over occasions were attributed to the intervention. The obtained differences in teachers' goal orientation at the pretest and posttest were explained by several factor variables. Subsequently, differences in effects of the factor variables can be attributed to the intervention. These differences in effects between the pretest and posttest were obtained as additionally sampled output from the MCMC algorithm, and they were used to investigate the significance of effect differences between occasions. When considering the increase in the intercept at the post-occasion, a significant intervention effect of .36 was found (HPD: $[.14, .55])$. This means that goaloriented teaching improved for teachers with a monograde group, and a population average of years of experience, according to the perceptions of grade- 6 boys. According to the girls in this same grade, the intervention effect was slightly smaller (intervention effect $=.25, H P D$ : $[.05, .45])$. As far as the grade effect is concerned, this was strongest for students in grade 2 (intervention effect: .42, HPD: [.21, .62]), followed by students in grade 6 , grade 3 and grade 5. In grade 4, the smallest and also non-significant intervention effect was found (intervention effect $=.11$, HPD: $[-.09, .30])$.

It seems that students in a multi-grade group benefited most of the DBDM intervention as goal-orientation of their teachers improved with .51 (HPD: $[.22, .76])$. 

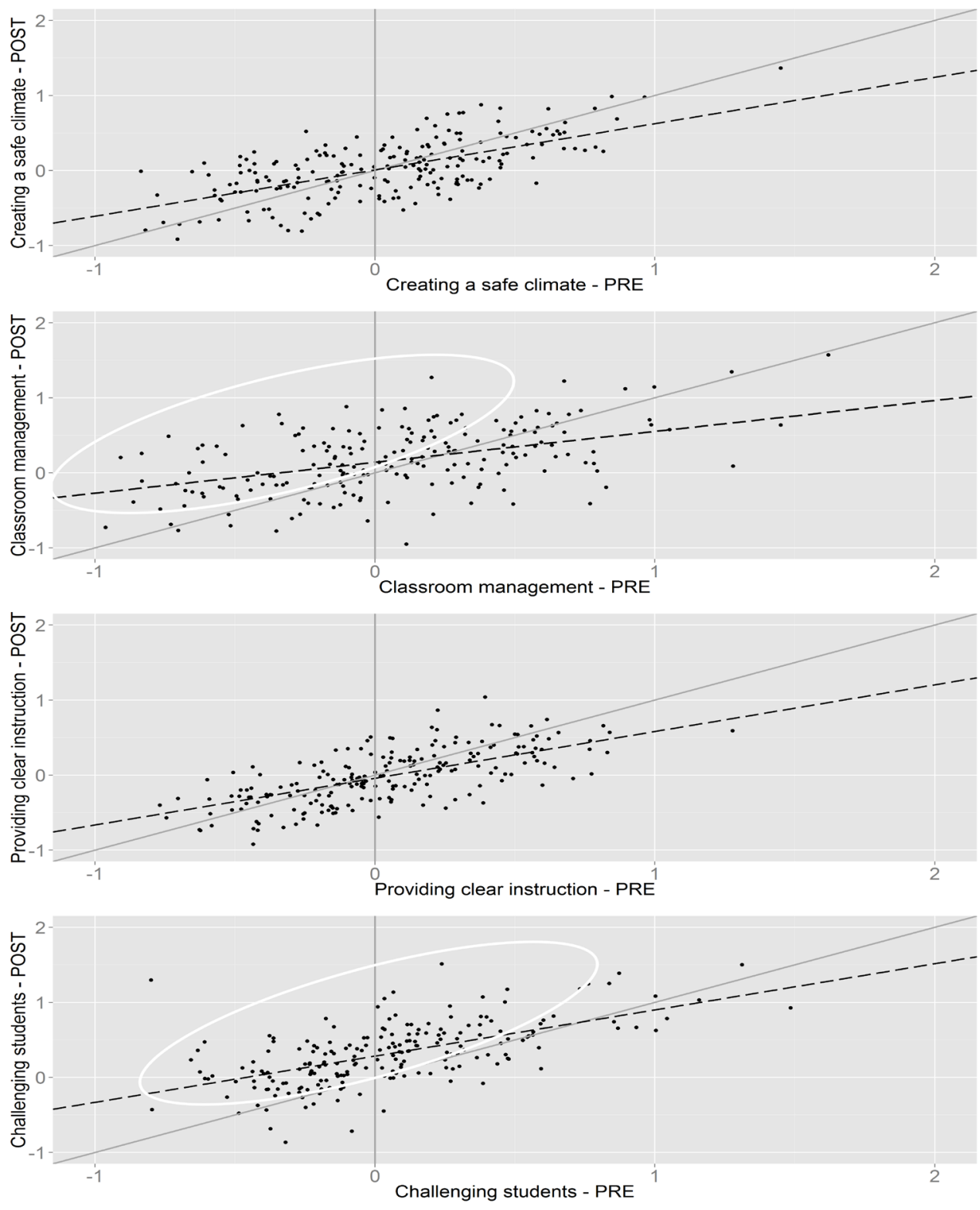

Figure 4. Teachers' factor scores on the pretest plotted against scores on the posttest for (from top to bottom): creating a safe climate, classroom management, providing clear instruction, challenging students 


\section{Analysis of the Other Four Teaching Quality Scales}

For the three basic teaching skills: 'safe climate' (5 items), 'classroom management' ( 7 items) and 'clear instruction' (10 items), no changes from pretest to posttest were expected, as these skills were not addressed during the intervention. We expected an increase of the fourth teaching skill 'challenging students' (5 items) which was, like goal-oriented teaching, considered to be a typical DBDM-teaching skill. For all four teaching skills, the same procedure was followed as for goal-oriented teaching, to estimate changes from pretest to posttest and to investigate the influence of background variables. In Figure 4, for each scale teachers' factor scores on the pretest are plotted on the posttest. A summary of the results is given for each scale below. In Appendix C, a detailed description of the findings is presented.

Creating a safe climate. The empty model for the safe climate scale revealed no changes from pretest to posttest suggesting that overall teachers' climate remained stable according to students. Results of the final model showed that at both the pretest and the posttest, students in grade 2 were most positive about their teachers' safe climate. Furthermore, girls tended to rate their teachers higher than boys. This gap between student ratings of boys and girls decreased significantly from pretest to posttest, but at the posttest girls were still more positive about their teachers than boys. Finally, the more experienced a teacher was, the lower (s)he scored on the safe climate scale.

Classroom management. Unexpectedly, based on the empty model it could be concluded that on average students' perceptions of their teachers' classroom management skills slightly improved, reflected by a small but significant positive intervention effect ( $\gamma_{0}^{\text {post }}$ $=.13, S D=.04, H P D:[.04, .20])$. In particular, teachers who scored below average at the pretest had higher student ratings at the posttest (see Figure 4: identified with an ellipse in the second subplot). However, after controlling for grade no significant differences were found between pretest and posttest. This might be caused by the fact that students in grade 2 scored their teacher significantly higher on both the pretest and posttest compared to the other grades. Additionally, teachers who changed groups had slightly higher classroom management skills at both the pretest and posttest according to their students compared to teachers who remained in the same group. No differences in the effects of explanatory variables were found between pretest and posttest.

Providing clear instruction. In contrast with the previous scales, no difference between grades was found for 'clear instruction' at the pretest, suggesting that a teacher's score on the clear instruction scale was not influenced by the teaching grade. However, there was variation between grades at the posttest and, on average, teachers in grade 2 received higher student rates concerning their teacher's instruction than teachers in grade 4. Additionally, at the pretest, girls tended to be more positive about teachers' instruction compared to boys, however this gap between boys and girls decreased significantly (mean difference $=-.12, S D=.04, H P D:[-.21,-.04])$ from pretest to posttest. Finally, at the pretest, a negative effect of teacher experience suggested that the more experienced the teacher, the lower students rated her/his instruction, however, this effect had faded prior to the posttest.

Challenging students. The empty model of the 'challenge students' scale revealed that teachers improved significantly in the degree to which they challenged their students, reflected by a $\gamma_{0}^{\text {post }}$ of .21 (SD $=.04$, HPD: $\left.[.12, .29]\right)$. This result is illustrated in Figure 4: most of the teachers (scoring below or above average at the pretest), scored higher at the posttest (this is reflected by the ellipse in the fourth subplot). Like in the case of goal-oriented teaching, teachers in multi-grade groups scored lower on the 'challenge students' scale at the pretest than teachers in monograde groups. After a year of DBDM intervention, no significant 
differences between multi-grade and monograde teachers were found, suggesting that the teachers in multi-grade groups benefitted much from the intervention. An intervention effect was also found for teachers who taught in grade 2 (despite that students in grade 2 had more positive perceptions compared to students in grade 6 at both the pretest and the posttest), 3 and 5. No effects were found for teachers in grades 4 and 6 . Finally, girls were more positive than boys at the pretest, however this effect disappeared at the posttest.

\section{Conclusions and Discussion}

DBDM requires that teachers systematically analyze student performance data, set goals based on such data, make instructional plans to accomplish these goals, and execute these plans in order to improve student performance. To effectively implement DBDM, teachers need to master various competences. In most of the available studies, the influence of DBDM interventions on teachers' knowledge, attitudes, and skills outside of the classroom was investigated (e.g. Marsh, 2012; Staman, Visscher, \& Luyten, 2014). Even though what teachers do inside the classroom is probably the most influential component of DBDM, research into teacher DBDM-skills inside the classroom is scarce. The current study contributes to the DBDM knowledge base by showing the effects of a DBDM intervention on teaching quality. Moreover, in doing so it has provided insights into using student perceptions as an efficient and valid way of measuring teaching quality in elementary education. Furthermore, it has shown an alternative to the traditional change score methods for analyzing the pre-posttest data. We will elaborate more on these three results and thereafter present some suggestions for future research.

\section{The Influence of the DBDM Intervention on Teaching Quality}

In general, our findings suggests that the basic teaching skills remained stable after one year of the DBDM intervention, while the specific DBDM-skills improved significantly in the eyes of the students. Student perceptions becoming more positive between pretest and posttest in combination with the fact that students seemed to agree more strongly with each other, suggests that students perceived an improvement in both the teachers' 'goaloriented teaching', and the degree to which teachers 'challenged students' during the DBDM intervention. Teachers seemingly increased the extent to which they made clear to their students what they were learning and why. Furthermore, they also monitored, together with their students, how the students were progressing during the lessons. Moreover, students felt more challenged by their teachers, meaning that the teacher encouraged them to a greater extent to do the best that they could. This is in line with the aim of DBDM to accomplish maximum achievement levels for all students.

A more detailed look into the intervention effects indicates that the improvement for 'goal-oriented teaching' and 'challenging students' applies to all grades, except for grade 4. For goal-oriented teaching, a possible explanation may be that grade 4 teachers scored relatively high on the pretest, and therefore improved less compared to their colleagues in other grades (they have approximately the same posttest scores as teachers in grades 3, 5 and 6).

Our findings reveal that the intervention was especially beneficial for teachers in multi-grade groups. In our sample, approximately $15 \%$ of all teachers taught a multi-grade group. At the pretest teachers in multi-grade groups scored lowest for the two DBDM-skills 'goal-oriented teaching', and 'challenging students'. However, the intervention effects for 
this group of teachers proved to be the highest, thus, the gap between teachers in multigrade groups and monograde groups almost disappeared. Teachers in multi-grade groups have to divide their attention between two or more groups, hence, it is harder for them to distribute their time equally between all students (Little, 2001, 2006). Apparently, teachers in multi-grade groups initially challenged their students less, and rarely used goal-oriented instructional strategies. The intervention may have supported them in becoming more aware of the importance of these two teaching skills and encouraged them to apply these more readily.

With regard to teacher experience, our findings at the pretest showed that for 'creating a safe climate', 'providing clear instruction', and 'goal-oriented teaching', novice teachers scored higher compared to experienced teachers. This is not in line with existing research-for instance, Van de Grift, van der Wal and Torenbeek (2011) found that teachers at the start of their career score much lower than more experienced teachers. At the posttest no effect of teacher experience was found for 'providing clear instruction' and 'goaloriented teaching', suggesting that the differences between novice and experienced teachers decreased between the two tests.

\section{Using Student Perceptions to Measure Teaching Quality in Elementary Education}

Although the use of student perceptions in elementary education at present is not widespread, our findings indicate that student perceptions are useful for evaluating teaching quality in elementary education. We also found interesting relationships between rater characteristics and their ratings. On all scales except 'classroom management', girls tended to rate their teachers slightly more positively than boys, which is consistent with other research (e.g. Koth, Bradshaw, \& Leaf, 2008). Interestingly, the effect of gender decreased significantly between pretest and posttest across all scales.

The second background variable related to student ratings was their grade. For all five teaching skills, findings suggest that students in grade 2 have more positive perceptions of their teachers on both the pretest and posttest measures compared to students from higher grades. These findings could imply that teachers in these grades are highly proficient at all five measured skills. A more plausible explanation, however, could be that students at the age of 7 and 8 years in general tend to give more positive ratings of their teacher. It is known that students' response style can differ because of their age, and that younger students seem to be more inclined to use the extremes in the rating scale (Vaerenbergh \& Thomas, 2013). However, this does not cause problems when investigating our main topic of interest: change. The results clearly indicate that also for grade 2 student ratings of their teachers were even more positive at the posttest.

\section{A New Way to Analyze Teaching Quality Data: A Pre-Post Multilevel IRT Approach}

A pre-post multivariate multilevel IRT model has been developed, which exceeds the traditional change score method and regressor variable approach. This model supports estimating effects of explanatory variables on the pretest and on the posttest at different modeling levels, while controlling for measurement errors at both time points. The multivariate modeling of pretest and posttest scores makes it possible to compute occasion-specific effects of explanatory variables, while accounting for occasion-specific error distributions. The developed Bayesian estimation method (MCMC) enables the joint estimation of all model parameters including the differential effects of explanatory variables at the pre- and posttest occasions. 


\section{Areas for Future Research}

In the literature, there are a few large-scale studies on data-based decision making in education. May and Robinson (2007), described a large-scale empirical study, where schools were randomly assigned to a control and treatment group. Quint, Sepanik, and Smith (2008) described an intervention in 21 public schools, where third and fourth grade students' reading comprehension were assessed throughout the school year. Henderson, Petrosino, Guckenburg, and Hamilton (2007) assessed, four times during a school year, the mathematic abilities of eight grade students in eight schools. These studies used an interrupted timeseries design to collect the data. Series of measurements were obtained before and during the intervention, and a change (discontinuity) in the time series provides evidence of an intervention effect. It turns out that in a large-scale setting, including a control group is often impossible. In this DBDM intervention study, it proved to be impossible to include a control group of 37 comparable schools who were willing not to participate in some type of professional development trajectory for a two-year period, and to provide the required data on teaching quality. Nevertheless, we believe it is possible to make inferences about changes in teaching quality during the DBDM intervention.

As argued by Shadish, Kyse, and Rindskopf (2013), Shadish and Sullivan (2011), and Sullivan, Shadish, and Steiner (2015), among others, single-case designs are becoming more prominent for testing intervention effects. What Works Clearinghouse (Kratochwill et al., 2010), and other institutions (see, Sullivan et al., 2015), have communicated standards that this single-subject design must meet in order to obtain valid inferences. It is commonly accepted that if statistical methods are used that can handle the complexity of the SSD data, credible and strong inferences can be made from a SSD study (Shadish, Cook, \& Campbell, 2002).

In order to make correct inferences in large-scale studies without controls, statistical methods for SSD data have to be improved. More research is needed to show the increased power of using multilevel modeling techniques. Another complex element is the way trend is modeled. Both parametric and non-parametric methods requires a sufficient number of repeated measurements to properly model change over time. From this perspective, the proposed multivariate multilevel model contributes in both ways to the methods for the statistical analysis of SSD data. By modeling the covariance structure between measurements, developments over time can be described without explicitly modeling trend components. This is advantageous when the number of measurements per single case is rather small. Furthermore, the autocorrelation between measurements can be modeled using a latent variable, where the trend is modeled over measurements.

In this study student perceptions were used to measure teaching quality. The students acted as raters for their teachers with respect to their teaching skills. The reliability estimates of the measurements showed that on average (across students) highly reliable measurements of teaching skills were obtained. As using student perceptions provides information about teaching quality 'through the eyes of students' it would be interesting to compare those perceptions with classroom observations by external raters, to see whether these two different measures lead to similar outcomes.

Furthermore, as the overall aim of DBDM is to accomplish maximum student achievement levels for all students, we recommend the investigation of the relationship between teaching quality (as perceived by students) and student outcomes: which aspects of teaching quality are most positively related with student achievement? Do, for example, teachers with higher goal-oriented teaching ratings also have better performing students? Or 
is providing clear instruction more related to how students' progress? 


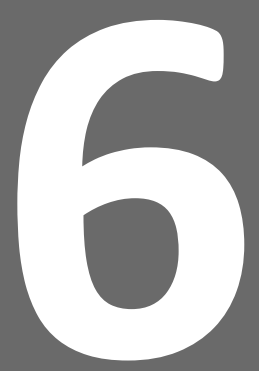

\section{State Transitions in}

\section{Educational Leadership}

During a Data-Based Decision Making Intervention

This chapter is a modified version of the submitted manuscript:

Van Geel, M., Keuning, T., Visscher, A.J., \& Fox, J.-P. (submitted). State Transitions in Educational Leadership During a Data-Based Decision Making Intervention. 


\section{Abstract}

Background. School leaders are assumed to be an important factor for the successful implementation of data-based decision making (DBDM). However, little is known about changes in leadership during the implementation of DBDM in schools.

Purpose. This study is aimed at investigating changes in leadership for DBDM when implementing DBDM by means of a schoolwide professional development trajectory.

Method. Before, during and after implementing a two-year intervention for DBDM, educational leadership was measured in 96 primary schools by means of teacher perceptions. Advanced analysis techniques were applied: educational leadership was classified based on multilevel latent class analysis, changes in leadership for DBDM and covariates related to those changes were modelled using multi-state modelling.

Findings. Results indicate that leadership for DBDM can be developed during an intensive intervention. In participating schools, leadership was stable (44\%) or improved (40\%). Stability was primarily found for schools with initial high leadership for DBDM, whereas improvement was most likely in schools with lower initial leadership. Furthermore, initial school leadership for DBDM was higher for female school leaders than for males, and for school leaders in small schools as compared to leaders in large schools. 
In education, there is a strong emphasis on the use of data for decision-making, assuming that this will lead to higher levels of student achievement (Hamilton et al., 2009; Marsh, 2012). School leaders are considered to be important in the successful implementation of reform in general, and data-based decision making (DBDM) specifically (Hallinger \& Heck, 2011; Ikemoto \& Marsh, 2007; Levin \& Datnow, 2012; Schildkamp \& Lai, 2013). In most studies, it is assumed that the school leader is a stable factor. However, a schoolwide reform can (and often is supposed to) influence leadership, but little is known about the changes in school leadership during school improvement processes. This study is aimed at measuring those changes when implementing DBDM by means of a schoolwide professional development trajectory. Can school leadership for DBDM be developed? Can we identify characteristics of schools, or school leaders, related to specific types of leadership, or to measured patterns of change?

In the following section, first DBDM and school leadership for DBDM will be discussed. After presenting the research questions, we will explore the challenges in measuring (changes in) educational leadership for DBDM.

\section{Theoretical Framework}

\section{Data-Based Decision Making}

Ikemoto and Marsh (2007) use the following broad definition of DBDM: "teachers, principals, and administrators systematically collecting and analyzing data to guide a range of decisions to help improve the success of students and schools" (p. 108). At the class, school and board (or district) levels, student and school performance data are supposed to be analysed, and decisions are supposed to be based on the outcomes (see also Figure 1). Since the aim of DBDM is to systematically maximize student achievement of all students, the focus in DBDM is on evaluating and analysing student performance data. Standardized test results are the starting point, but additional information also is gathered because no single assessment can provide all the information necessary to make informed decisions (Hamilton et al., 2009). Based on standardized test results, teachers come to understand their students' strengths and weaknesses, and they can use curriculum-based tests, classwork, homework and classroom observations to determine students' instructional needs in more detail.

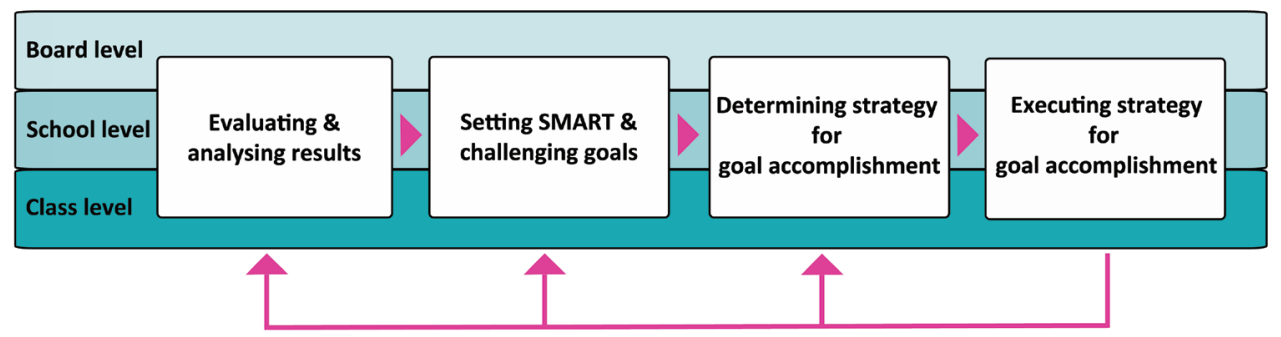

Figure 1. Schematic overview of DBDM

In the DBDM literature, the term 'decisions' implies a variety of actions that can be undertaken on the basis of data, such as: setting goals, adapting instruction, adapting the curriculum, evaluating the effectiveness of programs and practices, improving policy and reallocating time and resources as necessary (Earl \& Katz, 2006; Hamilton et al., 2009; Ikemoto \& Marsh, 2007; Mandinach, Gummer, \& Muller, 2011). In Figure 1, 'decisions' are decomposed into 
'setting goals' based on the data, and 'determining strategies' in order to achieve those goals.

The goals set and the strategies chosen for goal accomplishment vary according to the level of decision making. At the group level, teachers can use student performance data to differentiate instruction (Dunn, Airola, Lo, \& Garrison, 2013). Teachers first set goals in terms of desired achievement gains or skill attainment. To accomplish those goals, they can (for example) decide to use a specific instructional strategy, or to form a separate group of students to work on improving a specific skill. At the school and board levels, data can be used to highlight specific areas for improvement in the school(s), and the strategies chosen often comprise policy decisions such as the allocation of resources, or a modification of the curriculum. Mandinach (2012) rightly argues that data use to inform instructional decision is nothing new. Teachers collect information about their students all the time: they ask questions, observe students, and examine students' work. Mostly, teachers process this information to help them make informed decisions. However, this may not always be done systematically. Also at school level, DBDM is not implemented systematically - as for example is shown in the report of the Dutch Inspectorate, which stated that in the school years 2013-2014 only $34 \%$ of Dutch primary schools scored a 'sufficient' on all aspects of DBDM (Inspectie van het Onderwijs, 2015).

\section{Leadership for DBDM}

As Hallinger and Heck (2011) noted, "leadership acts as a catalyst for school improvement, both by initiating change and shaping a coherent focus on learning in schools" (p. 22). From initiating the improvement process to fulfilling practical preconditions, determining the vision, and influencing motivation and learning - school leaders can play an important role in the successful implementation of systematical DBDM in their school. School leaders are assumed to be an important factor in the successful implementation of DBDM in their schools (Ikemoto \& Marsh, 2007; Levin \& Datnow, 2012; Schildkamp \& Lai, 2013).

At the practical level, school leaders can fulfil the preconditions for DBDM such as the selection of a proper student monitoring system (Schildkamp \& Lai, 2013), and providing teachers with time for DBDM activities and collaboration (Cosner, 2012; Datnow, 2011; Ikemoto \& Marsh, 2007; Schildkamp \& Lai, 2013). This also was found in a study by Wayman, Cho, Jimerson and Spikes (2012), in which educators in schools with principals who facilitated data use showed positive attitudes and used data to support educational practice. In schools in which principals did not employ strategies to facilitate data use, educators' attitudes were low and data were not used to improve practice (Wayman et al., 2012).

At the cultural level, the vision and norms promoted by a school leader can influence the data-based culture of a school (Coburn \& Turner, 2011; Marsh, 2012). School leaders with a strong DBDM-vision, promoting norms of openness and collaboration enable data use (Ikemoto \& Marsh, 2007). It is important that they do not use data to shame and blame, but to improve the educational system of the school (Schildkamp \& Lai, 2013). School leaders have a powerful role in "setting the tone, directions and expectations of data use" (Anderson, Leithwood, \& Strauss, 2010, p. 323). By participating in data use routines themselves, using data to set performance goals at the school level, and to decide on strategies to achieve these goals, school leaders can serve as a role model for the entire team (Coburn \& Turner, 2011; Copland, Knapp, \& Swinnerton, 2009; Marsh \& Farrell, 2014).

Timperley (2008) stressed the key role of the school leader in influencing the motivation, attitude, knowledge and skills of the team members regarding DBDM. Leaders can promote and organize the professional learning and development of teachers, and should 
be actively involved in learning and in improving their own professional competences too.

Several leadership studies point to the importance of principal stability in sustaining change for school improvement (Fink \& Brayman, 2006; Hallinger \& Heck, 2011; Leithwood, Harris, \& Hopkins, 2008). In general, 'stability' then refers to the same person fulfilling the principal position over time. This person then is assumed to assure organizational consistency, strengthen the school's infrastructure and (re)shape the school culture. However, little is known about the changes in the content of school leadership during school improvement processes such as the implementation of DBDM. This could imply that it is assumed that the way school leaders fulfill their role and task will be stable over time, but it could also be argued that this person is going through a similar development process as the entire school and would therefore become more DBDM oriented as a leader as well.

The present study is aimed at investigating (changes in) school leadership prior to, during, and after the implementation of DBDM by means of a comprehensive professional development trajectory for all school team members. In the subsequent section, the intervention as implemented in the schools participating in the DBDM project is described and next, the research questions are presented. Finally, some methodological issues related to measuring (changes in) educational leadership are discussed.

\section{The DBDM Intervention}

The Dutch Ministry of Education supports schools to implement DBDM in their organization. Implementing DBDM can be regarded as a comprehensive school reform process, and schools need support in accomplishing this. Professional development activities can be helpful in this matter, for example in acquiring the required DBDM knowledge and skills (Mandinach, 2012; Timperley, 2008). At the University of Twente, a professional development trajectory for DBDM was developed and re-adjusted based on a pilot study. In the school years 201112-13 and 2012-13-14, the intervention was implemented in 53 and 48 schools respectively. The intervention was aimed at implementing and sustaining DBDM in the schools, following the cycle as presented in Figure 1.

During the intervention all team members learn how to analyze and interpret student achievement data, and performance goals are set at the school level as well as at the group level and the individual student level. Both teachers as well as school leaders decide on how to accomplish those goals. The team regularly discusses progress towards the goals set and evaluates the effects of the approach chosen.

As described in the previous, educational leadership is assumed to play an important role in establishing and sustaining DBDM in the school organization. Before and during the intervention, attention was therefore paid to supporting school leaders in being or becoming educational DBDM leaders. This was done by organizing meetings in which the project coach met with school leader and academic coach at every participating school. In these meetings, the importance of fulfilling the practical preconditions was stressed, progress and goals at the school level were discussed, and school leaders were supported in preparing team meetings during which student achievement in the various grades was evaluated, and educational strategies were discussed. Furthermore, school leaders were provided with an instrument and accompanying training activities to observe teacher classroom behavior, in order to enhance school leader involvement in monitoring teaching quality.

\section{Research Questions}

As it was assumed that school leadership is important for implementing and sustaining 
DBDM in the school organization, although the primary focus of the intervention was not at developing leadership for DBDM, it was supposed that the intervention would support school leaders in being or becoming educational leaders for DBDM. Therefore, we expected that school leaders would become more DBDM oriented in their leadership. However, we were also interested in their initial classifications, since we especially were expecting changes for school leaders who were less DBDM oriented at the start of the intervention. It would be desirable to improve in case of low levels of leadership for DBDM, whereas stability would be sufficient for school leaders who already showed strong leadership for DBDM. Thus, our main research question was: how does school leadership for DBDM develop during the intervention?

Furthermore, we were interested in the school characteristics and school leader characteristics related to initial leadership classifications, and related to patterns of change - will initial class assignment and/or transition probabilities differ for school leaders with different characteristics?

\section{Measuring (changes in) Educational Leadership}

Measuring educational leadership. In most leadership studies, the latent variable 'educational leadership' is measured by means of perceptions - either based on school leader self-reports, or teacher questionnaires. Examples of self-report are school leaders' perceptions of the features of their own leadership (for example in Urick \& Bowers, 2014), or school leaders' estimation of the allocation of time to a variety of tasks (Goldring, Huff, May and Camburn 2008). In most of the studies selected for the meta-analysis on leadership effects by Robinson, Lloyd and Rowe (2008), teacher perceptions were used to measure educational leadership (in 17 out of 27 studies this approach was taken). Teacher perceptions are for example used for principal evaluation (Goldring, Mavrogordato, \& Haynes, 2014; Liu, Springer, Stuit, \& Wan, 2014), to determine school leader effectiveness (for example in Porter et al., 2010), or to identify leadership practices (e.g. Leithwood, Patten, \& Jantzi, 2010).

A drawback of school leader self-reports is that these are likely to overestimate their leadership actions and qualities. Teachers usually are more critical about the school leader (Anderson et al., 2010; Desimone, 2006), and Hallinger (2010) stated that teacher perceptions showed a closer match to independent sources of evidence, leading to the conclusion that teacher perceptions are the "preferred source of data on the principal's instructional leadership for both research and evaluation purposes" (Hallinger, 2010, p. 293). For the current study, team member perceptions were therefore used to identify leadership for DBDM.

Measuring changes. Studies into educational leadership are often aimed at revealing variables influencing leadership, at measuring leadership effectiveness, or at understanding the paths through which leadership influences dependent variables such as student achievement (Hallinger, 2010). Longitudinal studies are scarce, the review of 130 doctoral studies using the Principal Instructional Management Rating Scale covering the period 1983 - 2010 did not include one reciprocal, longitudinal or experimental study (Hallinger, 2010). In the current study, we are interested in changes in educational leadership within schools during a two-year intervention. Multi-state modelling (MSM) can be used to model changes in principals' educational leadership over time. MSM can be used to describe a process in which school leaders move through (ordered) latent states, representing ordered levels of educational leadership, as depicted in Figure 2. Arrows with straight lines represent possible 
transitions from one state to the next, dashed arrows represent the implied relationships of a latent class measurement model.

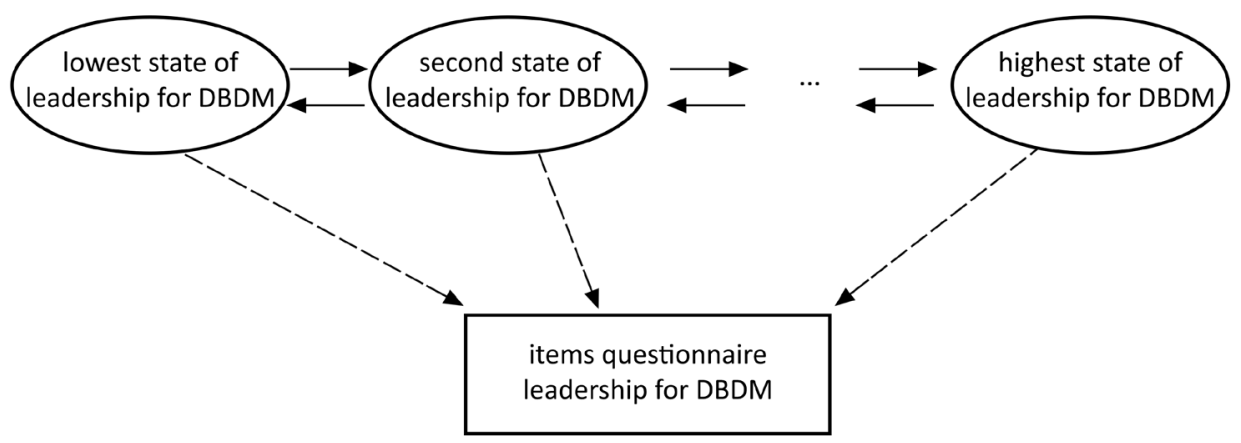

Figure 2. Proposed multi-state model for latent classes

Until now, MSM is mostly applied in medical studies, for example in order to model various states of diseases by stages of severity (e.g. Hout, Fox, \& Klein Entink, 2015). In the context of the current study, different states of educational leadership for DBDM can be regarded as (ideally) subsequent stages in school leader development. In a multi-state model, transition rates from state to state can be modelled, and covariates can be introduced to explain transition intensities (Jackson, 2014).

Multilevel latent class analysis. In most of the studies using teacher perceptions, the observed scores reflect the degree to which respondents agree with the items defining the leadership scale, and teachers' scores are aggregated to the school level to determine the level of instructional leadership (e.g. Heck \& Hallinger, 2009; ten Bruggencate, 2009). Although often used, calculating mean scores might not be the optimal method for characterizing school leadership. The drawback of using mean scores, at both the individual as well as the school level, is that this suggests that the (mean) observed score has the same meaning for all response patterns, which does not necessarily needs to be the case. Furthermore, the mean score summarizes the response-pattern information, and ignores differences between response patterns leading to the same mean score. In longitudinal data analysis, the mean score as an outcome measure can lead to substantial bias in parameter estimates leading to incorrect statistical inferences (Gorter, Fox, \& Twisk, 2015).

Only recently, Latent Class Analysis (LCA) is being applied in the field of educational research. LCA is used to identify groups of cases by patterns in the responses, therefore this method is well-suited for discovering different types of school leaders (Urick \& Bowers, 2014). For example, in Urick and Bowers (2014) it was shown that school leaders showed high scores on specific tasks and leadership characteristics, and low on teacher influence - or the other way around. Without taking the response patterns into account, indicating which items were scored high and low, 'controlling' and 'balkanizing' leaders could not be distinguished from each other - both showed similar mean scores. LCA enabled the researchers to identify these different types of school leaders.

The use of teacher perceptions for measuring school leadership requires a multilevel approach, as team members are nested within schools. Traditionally, the analyses of these relationships are approached by aggregating individual-level predictors to the group (school) 
Table 1. Sample characteristics of school leaders and schools

\begin{tabular}{|c|c|c|c|c|}
\hline & $\begin{array}{c}\text { At T1 } \\
(\mathrm{N}=92)\end{array}$ & $\begin{array}{l}\text { With stable } \\
\text { school leader } \\
(\mathrm{N}=86)\end{array}$ & $\begin{array}{c}\text { Stable school } \\
\text { leader, data on } \\
\text { both } \mathrm{T} 1 \text { and } \mathrm{T} 3 \\
(\mathrm{~N}=80)\end{array}$ & $\begin{array}{l}\text { Overall } \\
(\mathrm{N}=96)\end{array}$ \\
\hline \multicolumn{5}{|l|}{ School leader } \\
\hline \multicolumn{5}{|l|}{ Gender } \\
\hline Male & $48(52.2 \%)$ & $44(51.2 \%)$ & $41(51.3 \%)$ & \\
\hline Female & $44(47.8 \%)$ & $42(48.8 \%)$ & $39(48.8 \%)$ & \\
\hline \multicolumn{5}{|l|}{ Age } \\
\hline 40 or younger & 9 (9.8\%) & $10(11.6 \%)$ & $8(10 \%)$ & \\
\hline $41-50$ & $18(19.6 \%)$ & $15(17.4 \%)$ & $14(17.5 \%)$ & \\
\hline 51 and older & $62(67.4 \%)$ & $61(70.9 \%)$ & $58(72.5 \%)$ & \\
\hline Unknown & $3(3.3 \%)$ & $0(0.0 \%)$ & $0(0.0 \%)$ & \\
\hline \multicolumn{5}{|l|}{ Education } \\
\hline Higher education & $30(32.6 \%)$ & $28(32.6 \%)$ & $27(33.8 \%)$ & \\
\hline Master's degree & $56(60.9 \%)$ & 55 (63.9\%) & $50(62.5 \%)$ & \\
\hline Unknown & $6(6.5 \%)$ & $3(3.5 \%)$ & $3(3.8 \%)$ & \\
\hline \multicolumn{5}{|l|}{ School } \\
\hline \multicolumn{5}{|l|}{ School size } \\
\hline Small $(<150)$ & $25(27.2 \%)$ & $28(32.6 \%)$ & $25(31.3 \%)$ & $28(29.2 \%)$ \\
\hline Medium (150-350) & $48(52.2 \%)$ & $44(51.2 \%)$ & $41(51.3 \%)$ & $49(51.0 \%)$ \\
\hline Large (>350) & 19 (20.7\%) & $14(16.3 \%)$ & $14(17.5 \%)$ & 19 (19.8\%) \\
\hline \multicolumn{5}{|l|}{ School-SES } \\
\hline High & $24(26.1 \%)$ & $19(22.1 \%)$ & $19(23.8 \%)$ & 25 (26.0\%) \\
\hline Medium & 47 (51.1\%) & $47(54.7 \%)$ & $43(53.8 \%)$ & 49 (51.0\%) \\
\hline Low & $21(22.8 \%)$ & $20(23.3 \%)$ & $18(22.5 \%)$ & $22(23.0 \%)$ \\
\hline \multicolumn{5}{|l|}{ Urbanization } \\
\hline Rural & $34(37.0 \%)$ & $35(40.7 \%)$ & $31(38.8 \%)$ & 37 (38.5\%) \\
\hline Suburban & $40(43.5 \%)$ & $36(41.9 \%)$ & $35(43.8 \%)$ & $41(42.7 \%)$ \\
\hline Urban & $18(19.6 \%)$ & $15(17.4 \%)$ & $14(17.5 \%)$ & $18(18.8 \%)$ \\
\hline \multicolumn{5}{|l|}{ Principal stability } \\
\hline Stable & & & & $86(89.6 \%)$ \\
\hline Changed: T1 - T2 & & & & $3(3.1 \%)$ \\
\hline Changed: T2 - T3 & & & & $8(8.3 \%)$ \\
\hline
\end{tabular}


level, and by using this school mean as an outcome. This way, the assumptions with regard to independent errors among individuals are violated and statistical power is increased artificially because the total sample size is not corrected for the dependency among the individual observations within a group. To overcome the problems of aggregation and disaggregation, multilevel latent class analysis can be applied (Bennink, Croon, \& Vermunt, 2013; Bijmolt, Paas, \& Vermunt, 2004; Vermunt, 2003).

MSM with latent class measurements. In sum: next to the classifications of school leadership, we are interested in changes in classifications of school leaders over time. In order to investigate changes in measured memberships of latent classes, multi-state modelling (MSM) can provide insight into patterns of change in general, and in relation to covariates. Since we expect school leaders to develop during the course of a DBDM intervention towards more DBDM oriented leaders, we want to model changes in measured memberships for a fixed number of (ordered) latent classes over time. By using the ordered latent classes as states, and by introducing covariates, different trajectories and their relations with covariates can be explored. To our knowledge, the application of MSM to the latent class analysis of educational leadership over time is new to the field of research into school leadership, and could help us understand more about the development of school leadership over time.

\section{Methodology}

\section{Sample}

Data on school leadership was collected as part of more comprehensive data collection related to the DBDM intervention. Although 101 primary schools participated in the intervention, due to planning issues two of those schools followed an adjusted version of the intervention, which did not cover two complete school years. Questionnaires were administered to all school team members (except for the school leader) in the 99 remaining schools, at the beginning of the intervention, half-way (after one year), and at the end (after two years) of the intervention. After removing measurements for schools with fewer than five team members at that measurement occasion, 96 unique schools with sufficient data remained: 92 schools at the first measurement occasion, 88 at the second, and 93 schools at the final measurement occasion. Characteristics of school leaders and schools can be found in Table 1. 'Principal stability' indicates whether the same person fulfilled the formal role of school leader during the two intervention years. If this was not the case, it is indicated when a change in school leader occurred. It can be noted that this does not add up to 96 but to 97 , since in one school, three persons fulfilled the role of school leader over the course of two subsequent school years.

A total of 1801 unique respondents completed the questionnaire. The questionnaire was completed by 1478 respondents at the first measurement occasion, 1225 at the second occasion, and 1320 at the final occasion. Characteristics of respondents per measurement occasion are presented in Table 2, just as characteristics of all unique respondents.

\section{Instruments}

The questionnaire about educational leadership was based on the instrument as developed and validated by Ten Bruggencate (2009), which in turn was based on the Principal Instructional Management Rating Scale (PIRMS) by Hallinger and Murphy (1985). The questionnaire comprised aspects of school leadership that can be regarded as important 
Table 2. Sample characteristics of respondents per measurement occasion

\begin{tabular}{|c|c|c|c|c|c|c|c|c|}
\hline \multirow{2}{*}{$\overline{\text { Gender }}$} & \multicolumn{2}{|c|}{$\begin{array}{c}\mathrm{T} 1 \\
(\mathrm{~N}=1,478)\end{array}$} & \multicolumn{2}{|c|}{$\begin{array}{c}\mathrm{T2} \\
(\mathrm{N}=1,225)\end{array}$} & \multicolumn{2}{|c|}{$\begin{array}{c}\mathrm{T} 3 \\
(\mathrm{~N}=1,320)\end{array}$} & \multicolumn{2}{|c|}{$\begin{array}{c}\text { Overall } \\
(\mathrm{N}=1,801)\end{array}$} \\
\hline & & & & & & & & \\
\hline Male & 182 & $(12.3 \%)$ & 143 & $(11.7 \%)$ & 132 & $(10.0 \%)$ & 200 & (11.1\%) \\
\hline Female & 1295 & $(87.6 \%)$ & 1079 & (88.1\%) & 1056 & $(80.0 \%)$ & 1469 & (81.6\%) \\
\hline Unknown & 1 & $(0.1 \%)$ & 3 & $(0.2 \%)$ & 132 & $(10.0 \%)$ & 132 & $(7.3 \%)$ \\
\hline \multicolumn{9}{|l|}{ Role } \\
\hline $\begin{array}{l}\text { Deputy school } \\
\text { leader }\end{array}$ & 22 & $(1.5 \%)$ & 6 & $(0.5 \%)$ & 9 & $(0.7 \%)$ & 25 & $(1.4 \%)$ \\
\hline Academic coach & 108 & (7.3\%) & 101 & $(8.2 \%)$ & 108 & $(8.2 \%)$ & 134 & $(7.4 \%)$ \\
\hline Teacher & 1240 & $(83.9 \%)$ & 1066 & (87.0\%) & 1131 & $(85.7 \%)$ & 1509 & (83.8\%) \\
\hline Other & 108 & $(7.3 \%)$ & 52 & $(4.2 \%)$ & 72 & (5.5\%) & 133 & (7.4\%) \\
\hline \multicolumn{9}{|l|}{ Education } \\
\hline Master's degree & 297 & $(20.0 \%)$ & 251 & (20.5\%) & 276 & $(20.9 \%)$ & 256 & (19.8\%) \\
\hline Higher vocational & 1002 & $(67.8 \%)$ & 794 & (64.8\%) & 876 & $(66.4 \%)$ & 1177 & (65.4\%) \\
\hline $\begin{array}{l}\text { Intermediate } \\
\text { vocational }\end{array}$ & 161 & $(10.9 \%)$ & 125 & $(10.2 \%)$ & 157 & $(11.9 \%)$ & 202 & $(11.2 \%)$ \\
\hline Unknown & 18 & $(1.2 \%)$ & 55 & $(4.5 \%)$ & 11 & $(0.8 \%)$ & 66 & $(3.7 \%)$ \\
\hline \multicolumn{9}{|l|}{ Age } \\
\hline$\leq 30$ & 355 & $(24.0 \%)$ & 309 & $(25.2 \%)$ & 342 & $(25.9 \%)$ & 472 & $(26.2 \%)$ \\
\hline $31-40$ & 376 & $(25.4 \%)$ & 287 & $(23.4 \%)$ & 326 & $(24.7 \%)$ & 440 & (24.4\%) \\
\hline $41-50$ & 300 & $(20.3 \%)$ & 246 & $(20.1 \%)$ & 278 & $(21.1 \%)$ & 358 & (19.9\%) \\
\hline $51-60$ & 398 & (26.9\%) & 335 & $(27.3 \%)$ & 350 & $(26.5 \%)$ & 455 & (25.3\%) \\
\hline$\geq 61$ & 46 & $(3.1 \%)$ & 32 & $(2.6 \%)$ & 23 & $(1.7 \%)$ & 56 & $(3.1 \%)$ \\
\hline Unknown & 3 & $(0.2 \%)$ & 16 & (1.3\%) & 1 & $(0.1 \%)$ & 20 & $(1.1 \%)$ \\
\hline
\end{tabular}

for DBDM, as described in the previous, and included 13 items, such as "The school leader emphasizes the importance of monitoring student achievement", "The school leader ensures a shared feeling of responsibility for the achievement of all students" and "The school leader encourages staff to work as a team". A translation of the Dutch questionnaire can be found in Appendix D. All team members (teachers of all grades, academic coach and people with other functions in the school such as teaching assistants and remedial teachers) were asked to answer the questions about the person who fulfilled the formal role of school leader at that moment. All items were scored on a four-point Likert-scale, ranging from 'totally disagree' to 'totally agree'. School leaders were asked for their demographic data, such as gender, age, and educational background. School level data such as urbanization and school-SES were extracted from the database of the Dutch School Inspectorate.

\section{Procedure}

Step 1: Identify multilevel latent class model. In the present study, data were collected within a hierarchically ordered system of team members nested within schools. As suggested by Bijmolt et al. (2004), a simultaneous multilevel latent approach was applied, in which memberships to classes at school level and at individual level were derived simultaneously.

The multilevel latent class model was estimated based on the data from all three measurement occasions, taking nesting within schools within measurement occasion into account. This enhances the interpretability of classes and subsequent transitions, since by doing so, the same response pattern (at the individual level) and proportion of individually 
assigned classes (at school level) led to the same class assignment at different measurement occasions. Thus, the latent classes have the same meaning over time.

For the multilevel latent class analysis, it is necessary to estimate the optimal number of ordered latent classes at the individual and school level. Information criteria were used to identify the optimal model for the observed data. Lukociene, Varriale, and Vermunt (2010) have investigated the performance of an array of information criteria when conducting multilevel latent class analysis. In their simulation study, the use of the number of lower- and higher-level units for computing the information criteria was compared. In order to simultaneously decide about the number of lower- and higher-level classes, the use of the Bayesian information criterion $(\mathrm{BIC}(K))$ using the number of higher-level units $(K)$ instead of the number of lower-level units $(N)$, is recommended (Lukociene et al., 2010). This BIC is expressed by the fit of the model and a penalty term to avoid overfitting the data. The penalty term includes the number of parameters $(r)$ and the sample size, represented by the number of higher-level units $\mathrm{K}$. Then, $\mathrm{BIC}(K)$ is expressed as:

$$
B I C(K)=-2 \log L+\log (K) r
$$

where $K$ is the number of higher-level units, in this case schools and $L$ the likelihood. The model with the lowest $\mathrm{BIC}(K)$ and therefore best fit was used to assign classes at individual level and at school level. The ML LCA model was estimated using Mplus 7.3 (Asparouhov \& Muthen, 2013; Muthén \& Muthén, 2004).

For each school at each measurement occasion, membership to the most likely class was estimated using the latent class posterior distribution obtained during the ML LCA estimation, in other words, for each school, the school's membership of the class for which the probability to be assigned to was largest, was selected (Asparouhov \& Muthen, 2013). Schools' memberships to the classes were used in the multi-state model.

Step 2: Analyze initial class assignment. In order to analyse the association between initially assigned memberships to classes and characteristics of school leaders and schools, Pearson's chi-square tests were performed. In case of significant differences, pairwise comparisons were made by applying Fisher's exact test.

Step 3: Multi-state modelling. In a multi-state model, transition probabilities for each pair of states are modelled in continuous time. A model is governed by a transition intensity matrix, in which it is defined which instantaneous transitions can occur. It is for example possible to specify whether a transition from state 1 to 3 can occur directly, or that the school must have passed through state 2 in between (Jackson, 2014). Estimated transition probabilities matrices within a given time can be extracted, providing the transition probabilities from each initial class to each class. So, for every point in time it can be estimated which proportion of school leaders with initial state $X$ will be assigned to state $X, Y, Z$ at that point in time.

\section{Results}

In the following section, the results of the multilevel latent class analysis, and subsequent multi-state models at the school level are presented. First, based on the response patterns, the individual classes were interpreted. Next, the school level classes were interpreted and 
labeled, based on the proportions of assigned classes at the individual level. Covariates at the school level were introduced to explore differences in initial class assignment. MSM was applied to investigate changes and patterns of change. In the final step, covariates were related to transitions.

\section{LCA - Model Selection}

The questionnaire consisted of 13 items, which were scored on a four-point Likert scale. To decrease the number of thresholds to be estimated, the answer categories 'totally disagree' and 'disagree' were recoded into one category, since there was only a small number of observations in the 'totally disagree' category (1,040 out of 55,299 answers, 1.9\%).

As described in the previous, different combinations of numbers of latent classes at the school level and the individual level were used to estimate a number of models. In order to enhance interpretability, a maximum of five classes at each level was chosen. In Table 3, the $\mathrm{BIC}(K)$ information criterion is presented, and based on this value the solution with five classes at the individual level and five classes at the school level was selected.

Table 3. $B I C(K)$ for each combination of latent classes at school and individual level

\begin{tabular}{lcccc}
\hline \multirow{2}{*}{ Individual level } & $\mathbf{2}$ & $\mathbf{3}$ & \multicolumn{3}{c}{ School level } & $\mathbf{4}$ & $\mathbf{5}$ \\
\hline 2 & 79787,52 & 79733,72 & 79744,47 & 79755,69 \\
3 & 74018,17 & 73920,19 & 73876,69 & 73882,85 \\
4 & 72615,66 & 72503,71 & 72456,00 & 72455,93 \\
5 & $\underline{71561,38}$ & $\underline{71415,49}$ & $\underline{71342,27}$ & $\underline{\mathbf{7 1 3 3 3 , 1 3}}$ \\
\hline
\end{tabular}

Note. Lowest in row: italic, lowest in column: underlined. Overall lowest: bold.

Each respondent was assigned to the individual class with largest class assignment probability. On average, this was a probability of .90 (range .36-1.00, $S D=.14, N=4023$ ). At school level, schools were assigned to the school class with largest class assignment probability. This was an average probability of .84 (range: .38-1.00, $S D=.18, N=273$ ).

\section{LCA - Interpretation of Classes at Individual Level}

At the individual level, five classes of school leaders can be distinguished. Based on the scoring profiles, we can order the classes based on the degree of educational leadership for DBDM as perceived by the respondents. As can be noted in Figure 3, the average response pattern is quite similar across classes, with an exception for individual class (IC) number 3. For example, in all classes the average score for item 10 ("the school leader guides and supports teachers in maximizing student achievement") was lower than for other items, and the average score for item 12 ("the school leader ensures a shared feeling of responsibility for the achievement of all students") was higher than for item 13 ("the school leader promotes that lessons are of high quality") in all classes. For IC3, the response pattern was very stable across items with a similar mean score for every item. 


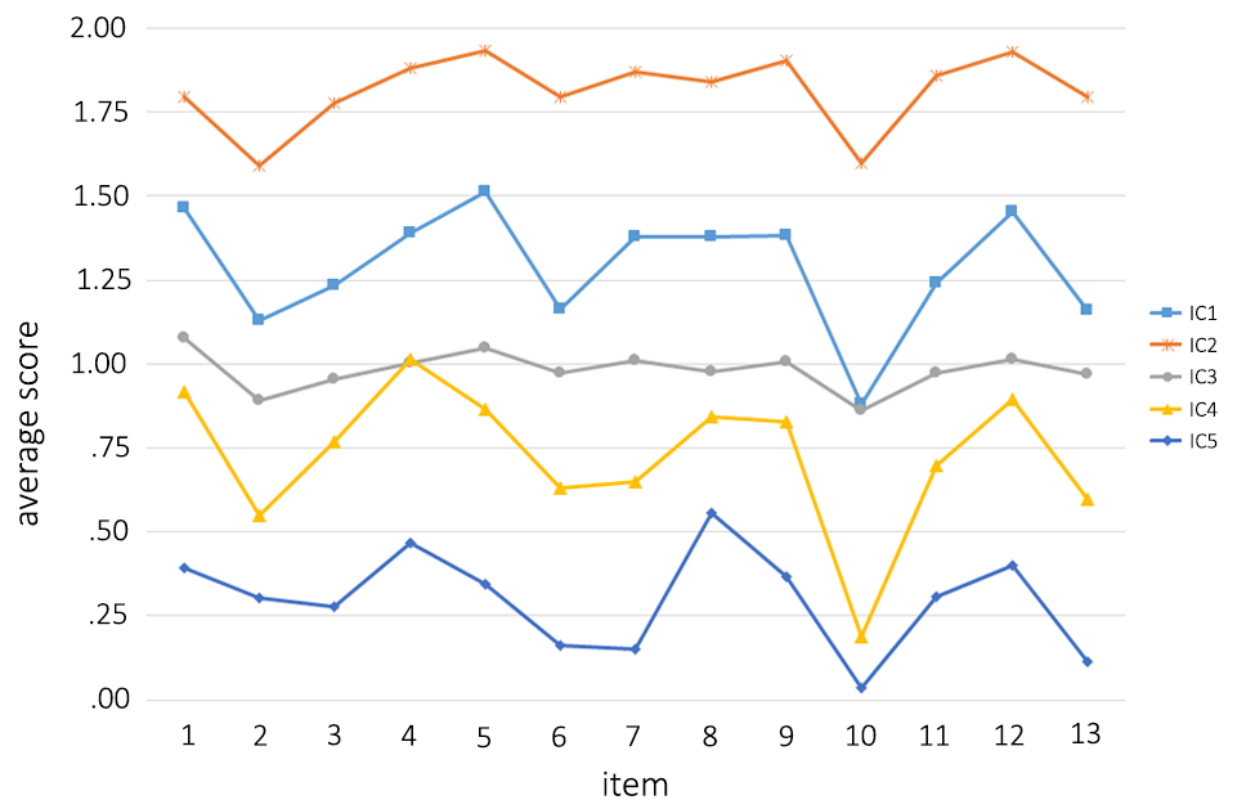

Figure 3. Average respondent item scores for assigned class at individual level

\section{LCA - Interpretation of Classes at School Level}

In order to interpret classes at the school level, we studied the proportion of respondents assigned to the individual classes in relation to the assigned school class. For interpretation purposes, we ordered the individual classes (IC) based on the scoring profiles as follows: IC5 - IC4 - IC3 - IC1 - IC2. In Table 4, the proportion of individuals assigned to each of those individual classes, per assigned class at the school level, is presented. School classes were ordered based on the proportion of respondents in the individual classes.

School class (SC) 1 is regarded as an indicator for the least DBDM oriented school leader, since most individuals in these schools were assigned to individual class 5 . In teams of schools assigned to SC2, respondents were assigned to individual classes 4, 3 and 1. SC5 represents school leaders regarded as most DBDM oriented, since most individuals in these schools were assigned to individual class1 and 2.

Table 4. Proportion of individuals assigned to individual class, per school class

\begin{tabular}{lccccc}
\hline & IC5 & IC4 & IC3 & IC1 & IC2 \\
\hline SC1 & $49 \%$ & $33 \%$ & $11 \%$ & $7 \%$ & $0 \%$ \\
SC2 & $10 \%$ & $54 \%$ & $17 \%$ & $15 \%$ & $3 \%$ \\
SC3 & $0 \%$ & $25 \%$ & $28 \%$ & $41 \%$ & $7 \%$ \\
SC4 & $3 \%$ & $8 \%$ & $60 \%$ & $20 \%$ & $8 \%$ \\
SC5 & $0 \%$ & $3 \%$ & $16 \%$ & $44 \%$ & $37 \%$ \\
\hline
\end{tabular}

From Table 5 it appears that the distribution of school leaders assigned to the classes for SC3 is quite stable across measurement occasions, and we can distinguish a proportional increase for SC4 and SC5 and a decline for SC1 and SC2. The five-class solution at the school level was the optimal solution, and at all measurement occasions there were school leaders 
assigned to all classes. We can therefore conclude that school leaders initially differed from each other (otherwise they all would have been assigned to the same initial class) and that there eventually still were differences between school leaders.

Table 5. Number of school leaders assigned to each latent class, per moment

\begin{tabular}{ccccccc}
\hline & SC1 & SC2 & SC3 & SC4 & SC5 & Total \\
\hline T1 & $13(14 \%)$ & $37(40 \%)$ & $22(24 \%)$ & $11(12 \%)$ & $9(10 \%)$ & 92 \\
T2 & $11(13 \%)$ & $31(35 \%)$ & $26(30 \%)$ & $7(8 \%)$ & $13(15 \%)$ & 88 \\
T3 & $7(8 \%)$ & $29(31 \%)$ & $22(24 \%)$ & $20(22 \%)$ & $15(16 \%)$ & 93 \\
\hline
\end{tabular}

\section{Exploring Initial Class Assignment}

We explored the assigned classes at the start of the intervention in relation to characteristics of schools and school leaders. Descriptives of school leaders and schools are presented in Table 1, descriptives of all 92 schools and school leaders at the first measurement occasion can be found in the first column.

Although no significant association between school size and initial class membership was found $\left(\chi^{2}(8)=14.58, p=.068\right)$, post hoc pairwise comparison revealed a significant difference (Fisher's exact test yields $p=.031$ ) between small and large schools. For large schools, 16 out of the 19 schools were initially assigned to SC1 or SC2 - indicating low levels of DBDM school leadership. For small schools, 10 out of 25 were assigned to SC4 or SC5, indicating that school leaders in small schools were more DBDM oriented the beginning of the intervention. No significant differences were found for urbanization or school-SES.

Next to characteristics of schools, school leader characteristics can be related to leadership for DBDM as well. Although we did not formulate hypotheses about the correlation between school leader characteristics and class membership, we explored personal characteristics in relation to initial leadership for DBDM. A significant difference was found for gender $\left(\chi^{2}(4)=10.22, p<.05\right)$. Although we do not have a theoretically grounded explanation, we know that female principals in general receive higher ratings on the Principal Instruction Management Rating Scale than males (Hallinger, 2010). This was again confirmed in our study: male school leaders were regarded as less DBDM oriented (32 out of 48 of the male school leaders were assigned to SC1 or SC2 at moment T1, 11 to SC3, and 5 to SC4 or SC5) than their female counterparts (15 out of 44 to SC5 or SC4, 11 to SC3 and 18 to SC1 or SC2 and at moment T1).

Based on school leader age, or with regard to a school leader's educational level, no significant differences in initial class membership were found.

\section{Multi-State Model - Transitions at School Level}

Since we were specifically interested in changes in school leadership, we only looked into transitions for schools in which the same person fulfilled the formal position of school leader over the course of the entire intervention. Descriptives of schools and school leaders in those 86 schools are presented in the middle column of Table 1.

School memberships to latent classes were estimated at fixed measurement occasions: the beginning of the intervention, at the end of the first intervention year (which was 10 months later), and at the end of the second intervention year (22 months after the baseline measure). A change in class membership has occurred at an unknown point in time, between two measurement occasions - which can be regarded as 'snapshots'. Multistate modelling was used to model changes in school status in continuous time, using a 
homogeneous continuous-time Markov model. Because the different classes at school level can be regarded as ordered levels of leadership for DBDM, it is reasonable to expect schools to evolve through adjacent states, without the possibility to 'skip' states. Therefore, we only allowed (instantaneous) transitions to adjacent states, indicating that a school had to move through class 3 in order to change from class 2 to 4 . This was accomplished by restricting some of the transition parameters of the MSM to zero. However, the 'observed' memberships of each school over time do not have to include all classes, since each school is only measured at (a maximum of) three fixed occasions.

Transition probabilities can be computed for every point in time, indicating the probability that a school leader who initially was assigned to class $X$, was assigned to class $Y$ after a specified number of months.

Table 6. Probability of being assigned to a latent class at the end of the intervention, given the initial assigned class

\begin{tabular}{lccccc}
\hline \multirow{2}{*}{ Initial class } & \multicolumn{5}{c}{ Class assigned to at the end of the intervention } \\
\cline { 2 - 5 } & SC1 & SC2 & SC3 & SC4 & SC5 \\
\hline SC1 & .20 & .44 & .22 & .09 & .05 \\
SC2 & .11 & .38 & .28 & .14 & .09 \\
SC3 & .04 & .20 & .33 & .22 & .21 \\
SC4 & .02 & .13 & .30 & .24 & .31 \\
SC5 & .01 & .06 & .22 & .24 & .47 \\
\hline
\end{tabular}

In Table 6, estimated transition probabilities after 22 months are shown for the population of school leaders, indicating the probability of being assigned to each of the states (latent classes) at the end of the intervention, given the initial state. For example, school leaders assigned to SC5 at the start of the intervention, will remain in SC5 with probability .47. For school leaders initially assigned to class SC1 the probability of being assigned to SC2 (.44) or SC3 (.22) is larger than being assigned to SC4, or SC5 (.09 and .05 respectively) at the end of the intervention. Furthermore, the probability of staying in the same class is largest for three out of five of the initially assigned classes. For school leaders initially assigned to one of the two other classes (SC1 or SC4), improvement is most likely: for school leaders initially assigned to SC4, the probability of changing to SC5 is larger than for staying assigned to SC4, and for school leaders initially assigned to SC1, the probability of changing to SC2, or SC3 is larger than staying in SC1. In Figure 4, overall transition probabilities are plotted over time for transitions that can be interpreted as improvement. It is interesting to see that the probability of larger improvement increases over time. For example, the probability of transitioning from SC1 to SC2 reaches its maximum after 15 months, the probability of following the transition pattern SC1 to SC2 is smaller after 22 months. This is due to the fact that improvement from SC1 to SC3, or even SC4, or SC5 is becoming more likely after 22 months. 


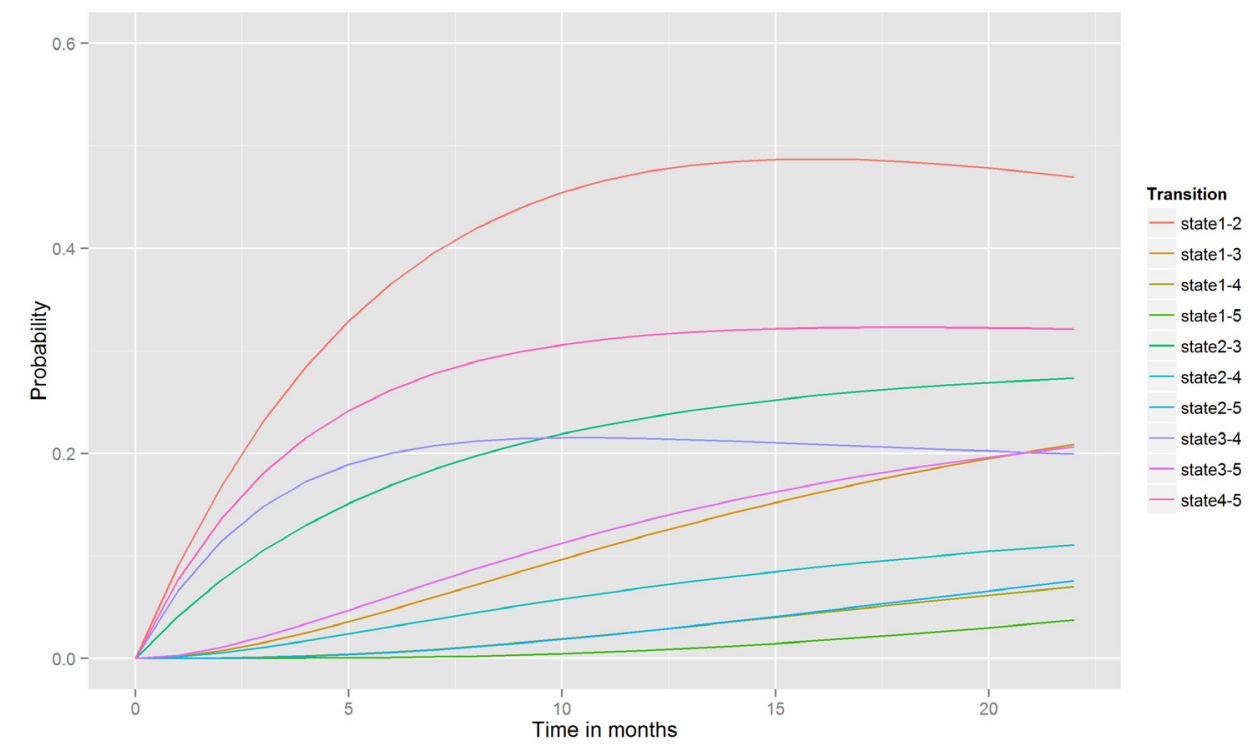

Figure 4. Transition probabilities over time, based on initial state

\section{Covariates Related to Transition}

In the previous, overall transition probabilities were described. In this section, we will explore differences in transitions for different characteristics of schools and school leaders. The sample and the number of transitions for different values of covariates were limited. Out of 80 school leaders, 35 were assigned to the same class at the beginning and at the end of the intervention, 32 school leaders were assigned to a higher class after the intervention, and thirteen were assigned to a lower class. Due to the large number of possible transitions, but small number of actual transitions, no significant effects of covariates on transition probabilities were found. However, in order to provide the reader with insight into changes and stability in relation to characteristics of school leaders and schools, we provide descriptives for different characteristics and transitions.

Since we discern five classes of school leadership which can be regarded as five increasing levels of leadership for DBDM, school leaders who were assigned to SC1 at the beginning of the intervention were more likely to improve than school leaders who were assigned to SC5 - there was no room for improvement for the latter kind of school leaders. This ceiling effect makes it difficult to interpret changes when comparing school leaders and schools based on background characteristics, especially when these showed different proportions of class assignment at T1. For example, when we look at the number of male and female school leaders who showed improvement from initial class SC1, this proves to be three for males and three for females, which is $7.3 \%$ and $7.7 \%$ of the total number of male and female school leaders respectively. This seems comparable, but when we take a closer look at the proportion of improvement from initial class SC1, we see that three out of six males and three out of three females showed improvement. Proportionally, females initially assigned to SC1 thus improved more than males (100\% versus 50\%). So, in order to interpret transitions, it is both informative to look at the overall proportion of change, but also to relate 
this to the initially assigned class. In the following section, for illustrative purposes, we will describe transitions in relation to each covariate.

School leader age. School leaders aged 51 and up were relatively often (9 out of 58) assigned to SC1 at the beginning, and 6 of them improved (4 to SC2, and 2 to SC3). Proportionally, school leaders aged 51 and up (26 out of 58) and 40 or younger (3 out of 8) were improving more often than school leaders aged $41-50$ (5 out of 14). Fifty percent of school leaders of age 40 or younger remained in the same class, for both higher age groups around $43 \%$ of school leaders was assigned to the same class at the beginning and the end of the intervention. The most remarkable figure with regard to school leader age, is that $36 \%$ of school leaders aged $41-50$ were assigned to a lower class, compared to $13 \%$ and $12 \%$ for the lower and higher age groups respectively.

School leader education. The initial distribution of class assignment was comparable for school leaders with a higher education and a master's degree. Furthermore, the proportion of change and stability, was almost comparable too. Where $46 \%$ of the school leaders with a master's degree was assigned to the same class at the beginning and at the end of the intervention, and $38 \%$ of them showed improvement, for school leaders with a higher education degree $48 \%$ showed improvement, and $33 \%$ of them remained in the same class. For school leaders with a higher education degree, 19\% was assigned to a lower class after the intervention, this was $16 \%$ for school leaders with a master's degree. However, school leaders with a master's degree showed a larger decline when initially assigned to SC5: out of four school leaders with a higher education background, two remained in SC5 and two went to SC4. Out of four school leaders with a master's degree, one remained in SC5, one went to SC4, one to SC3 and one to SC2.

School size. The majority of school leaders in large schools (71\%) was assigned to the same class at the beginning and end of the intervention, which was mostly SC2. If we look at school size in relation to initial class SC5, we see that the only large school initially assigned to SC5, still was assigned to SC5 after the intervention. Out of the four medium-sized schools initially assigned to SC5, three were still assigned to SC5 and the last one was assigned to SC4. Out of the four small schools initially assigned to SC5, only one remained in SC5. The other three declined to SC4, SC3 and SC2 respectively.

School-SES. For all categories of school-SES, approximately $43 \%$ of school leaders stayed in the same class. It is remarkable that none of the school leaders in low-SES schools was assigned to a lower class of leadership for DBDM after the intervention, so the majority of school leaders in those low-SES schools (56\%) was assigned to a higher class after the intervention.

\section{Conclusions and Discussion}

This study was aimed at investigating the development of leadership for data-based decision making, during a two-year schoolwide intervention. We assumed that school leaders would become more DBDM oriented in their leadership as a result of specific leadership-oriented intervention activities. Furthermore, we were interested in exploring characteristics of school leaders and schools related to initial leadership characteristics, or/and changes in leadership for DBDM.

\section{Initial Leadership for DBDM}

In order to gain insight into leadership for DBDM at the start of the intervention, we explored 
the relationship between leadership for DBDM, and characteristics of school leaders and schools. Although we do not have an explanation, just as in previous research, in this study it was found that female school leaders were regarded as more DBDM oriented than male school leaders. Initially, although not significant, younger school leaders appeared to be more DBDM oriented than school leaders aged 51 and older. A possible explanation for this might be that recent principal training is more aimed at leadership for DBDM. Furthermore, in large schools, school leaders were often regarded as less DBDM oriented than in small schools. This could be, because in a large school with an accompanying large team, it is more difficult for school leaders to guide the entire team towards DBDM. It might be that leadership tasks with regard to DBDM in those schools were (also) performed by for example deputy school leaders, or department leaders, enabling the school leader to focus more on other, for instance organizational, managerial, and administrative tasks.

\section{Changes in Leadership for DBDM}

It was expected that the intervention would support school leaders in being or becoming educational leaders for DBDM. Based on transition probabilities (as presented in Table 6) we conclude that remaining in or growing to a higher class of leadership for DBDM, was more probable than decreasing - except for SC5, where a ceiling effect might explain the probability of .47 for remaining in SC5. Furthermore, note that only 10 schools were initially assigned to SC5.

'Principal stability' is often regarded as an important condition for accomplishing and sustaining school improvement (Fink \& Brayman, 2006; Hallinger \& Heck, 2011; Leithwood et al., 2008), but we noticed that this 'stability' generally is about the same person fulfilling the principal position, and that little is known about changes within school leaders during a school improvement process such as the implementation of DBDM. Therefore, we specifically investigated whether school leaders in schools in which the same person fulfilled the formal role of school leader were also 'stable' in their leadership for DBDM. Our study confirms that a considerable minority of school leaders (35 out of 80 ) showed stable leadership for DBDM over time (as measured by means of team member perceptions).

Furthermore, a slightly smaller number (32 out of 80 ) of them showed improvement, indicating that participating in an intensive intervention, although not easy, can contribute to the development of leadership for DBDM. A more explicit focus on the development and sustainment of leadership for DBDM in the context of a schoolwide team intervention, could be fruitful for enhancing DBDM in the schools.

Finally, we were interested in the school characteristics and school leader characteristics related to patterns of change. Since our sample consisted of only a small number of schools, of which the majority showed stable leadership for DBDM over time, we were unable to uncover statistically significant differences in transition probabilities based on characteristics of school leaders and schools.

\section{Methodology}

Longitudinal studies into educational leadership are scarce. In the current study, leadership for DBDM was measured three times over the course of two school years. This type of leadership was measured by means of teacher perceptions, which is regarded the preferred source of data for leadership research, and analyzed by applying multilevel latent class analysis. Transitions in assigned classes were modeled by applying a multi-state model. Both ML LCA as well as MSM are advanced modelling techniques, suitable for the context and data 
structure of this study. To our knowledge, this is the first study in the field of educational research in which ML LCA and MSM are combined.

ML LCA was applied in order to take the nested structure of respondents in schools into account, and to possibly distinguish different types of leadership for DBDM based on response patterns, as opposed to using mean scores. MSM enabled the investigation of change in leadership for DBDM and relate this change to covariates. Unfortunately, due to the large number of possible transitions, and the considerable number of school leaders who did not show change, it was impossible to identify statistically significant deviances in transition probabilities based on covariates in this study.

\section{Limitations}

In the current study, we were interested in changes in leadership for DBDM during an intervention. Almost half of the school leaders was assigned to the same class at the beginning and end of the intervention, but also $40 \%$ showed improvement. Although the intervention was not primarily aimed at improving leadership for DBDM, it was clearly expected that this would happen due to the fact that school leaders and their teams were part of an intervention that was focused on implementing and sustaining DBDM in the entire school organization. A more explicit focus on school leader behavior could enhance improvement even more.

Furthermore, it would be interesting to include a control group of school leaders who are not part of an intervention in future studies. This way, we could gain insight into general patterns of change in leadership for DBDM, and check whether the improvements we found can be attributed to participating in the intervention. Furthermore, it is possible that school leaders in the current sample were already more DBDM oriented, otherwise they might not have applied their school for the intervention at all. It should be noted however that not all school leaders who were part of this study, were also the ones who had determined to participate in the intervention: this decision was sometimes made by the school board or district, or in some cases by the previous school leader. A new ML LCA, in a broader sample including a control group, would maybe discern more or other latent classes of leadership for DBDM and provide new insights into the relationship between both initial leadership for DBDM as well as transitions, and covariates.

\section{Implications}

In general, we can conclude that for many schools (35 out of 80 for which data on T1 and T3 was available) in which the same person fulfilled the formal principal position during the entire intervention, the school leader was assigned to the same class at the beginning and at the end of the intervention. This implies that $44 \%$ of the school leaders did not change their leadership for DBDM. Based on the transition probabilities as presented in Table 6, we can conclude that the probability of remaining in the same class is largest for SC5. This is positive since this is the class representing the most DBDM oriented school leader, and almost half of those most DBDM oriented school leaders was characterized this way after the intervention too. For school leaders initially assigned to class SC1, the probability of improvement is .80, which is also very promising for practice, since this means that for schools with initially low level of leadership for DBDM it is likely their school leader will become more supportive over time.

For school boards and districts, it is difficult to decide on how to handle school leaders who do not support their team for DBDM. School leaders initially assigned to SC1, only have a .20 probability of staying assigned to SC1 and to remain a non-supportive school leader during 
the implementation of an intensive intervention such as the one studied here. However, if we would state that being assigned to SC1 or SC2 is not desirable after an intervention of this kind, we have to conclude that only 36\% of school leaders initially assigned to SC1 and $51 \%$ of school leaders initially assigned to SC2 will improve to SC3, or higher. The question is whether more explicit attention for developing school leadership for DBDM would be fruitful, which could be evaluated in future research by studying the link between a more intensive, school leader aimed, intervention on the development of leadership for DBDM. Furthermore, our assumption that leadership in SC1 or SC2 would not be desirable, is not based on sound standard setting procedures.

Since school leadership for DBDM is expected to be positively associated with implementing DBDM and (ultimately) with raising student achievement, in a subsequent study we will investigate the relationship between leadership for DBDM and the overall effects of the intervention on student achievement. 
State transitions in educational leadership 


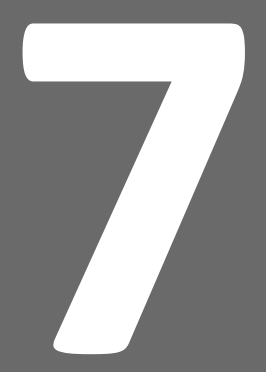

\section{Development of Schools' Data Culture during a} Schoolwide Data-Based

\section{Decision Making Intervention}

This chapter is a modified version of the submitted manuscript:

Van Geel, M., Keuning, T., Visscher, A.J., \& Fox, J.-P. (submitted). Development of Schools' Data Culture during a Schoolwide Data-Based Decision Making Intervention. 


\section{Abstract}

Background. It is often emphasized that a data culture, an achievement oriented culture that supports the use of data, is essential in order to implement and sustain data-based decision making (DBDM) in schools.

Purpose. The current study was aimed at investigating data culture in 96 schools before participating in an intensive DBDM intervention. Furthermore, since school leaders are regarded essential for both data culture and DBDM implementation, the relationship between leadership for DBDM and data culture was investigated.

Method. In 96 primary schools, data culture was measured by means of team member perceptions before, during and after implementing a two-year schoolwide professional development intervention for DBDM. School level data culture was defined based on multilevel latent class analysis, changes in data culture during the intervention, and covariates related to those changes, were modelled using multi-state modelling.

Findings. Results indicate that a school's data culture can be enhanced during an intensive DBDM intervention, and that schools with initial high levels of data culture maintained this high level. Characteristics of schools were not related to their initial data culture, but data culture was stronger for schools with more DBDM oriented school leaders. 
There is a world-wide increasing interest in using data in schools, for both accountability purposes (Lai \& Schildkamp, 2013) as well as for continuous school improvement (Lai \& Schildkamp, 2013; Mandinach, 2012; Kingston \& Nash, 2011). Data use, or data-based decision making (DBDM) is regarded as a way to inform teachers about students' needs, so they can adapt and adjust instruction based on such information, and also as a way to make policy decisions at the school level, with the ultimate aim to raise student achievement (Campbell \& Levin, 2008; Carlson, Borman, \& Robinson, 2011; Lai \& McNaughton, 2009).

Since DBDM is not common practice in Dutch primary schools (Inspectorate, 2015) and educators do not feel prepared to use data (Earl \& Fullan, 2003; Ikemoto \& Marsh, 2007), professional development in data use is regarded necessary (Desimone, 2009; Van Veen, Zwart, \& Meirink, 2011). A school culture that is achievement oriented and fosters data use is considered essential in order to implement and sustain DBDM in schools (Ingram, Louis, \& Schroeder, 2004; Mandinach, 2012b; Wayman, Midgley, \& Stringfield, 2006).

In the current study we therefore investigated the data culture in 96 primary schools previous to participating in an intensive DBDM intervention, and the development of this data culture during the intervention. Furthermore, since school leaders are regarded essential for both implementation of DBDM as well as for the establishment of schools' data culture, we related data culture to leadership for DBDM.

\section{Theoretical Framework}

\section{Data-Based Decision Making}

In education, there is a growing emphasis on the use of data to base decisions on, assuming that this will lead to increased student achievement. At the board level, school level, and classroom level, student and school performance data are supposed to be analyzed, and decisions are supposed to be based on the outcomes of those analyses (see also Figure 1). These 'decisions' can vary, for example from setting goals, or adapting the curriculum, to reallocation of time or other resources (Earl \& Katz, 2006; Hamilton et al., 2009; Ikemoto \& Marsh, 2007; Mandinach, Gummer, \& Muller, 2011). The aim of DBDM is to systematically maximize the achievement of all students (Ikemoto \& Marsh, 2007).

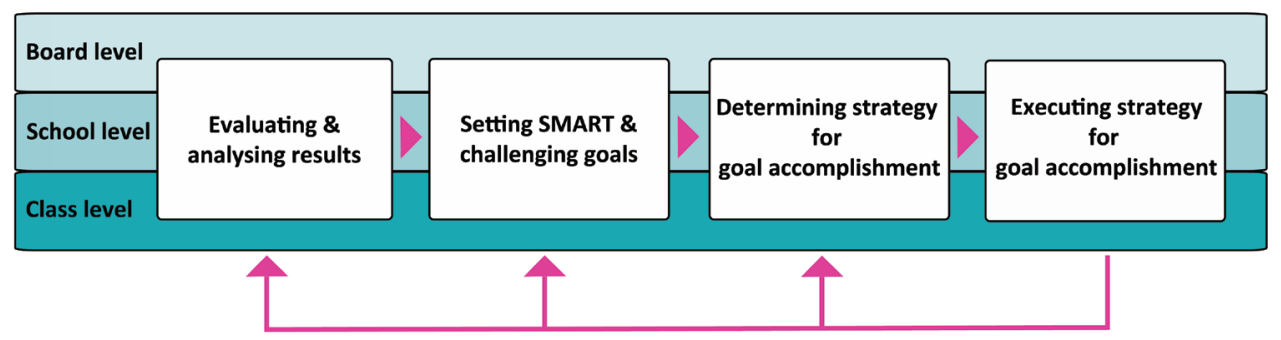

Figure 1. Schematic overview of DBDM

As Mandinach (2012a) argues, using data in order to inform instructional decisions is not something new. In practice, by asking questions, observing students, or examining students' work, teachers collect information about their students all the time and use this information to base their education on. However, this may not always be done systematically. At the school level, DBDM also is not implemented always in a systematic way - as for example becomes clear from the report of the Dutch Inspectorate. In the school years 2013-2014 only 
$34 \%$ of Dutch primary schools scored a 'sufficient' on all DBDM-indicators (Inspectie van het Onderwijs, 2015).

\section{School Culture for DBDM}

In the current literature on DBDM, it is often emphasized that a culture that supports the use of data is essential for the implementation of DBDM. For example in the IES practice guide (Hamilton et al., 2009), a panel of experts provided educators and schools with recommendations on how to use student achievement data to support instructional decision making, based on the expertise of the panelists, and research findings. Two of the five recommendations are specifically aimed at implementing DBDM at the school level: 'establish a clear vision for schoolwide data use' and 'provide supports that foster a data-driven culture within the school'. The concept of data culture is defined as:

"a learning environment within a school or district that includes attitudes, values, goals, norms of behavior, and practices, accompanied by an explicit vision for data use by leadership, that characterize a group's appreciation for the importance and power that data can bring to the decision-making process. It also includes the recognition that data collection is a necessary part of an educator's responsibilities and that the use of data to influence and inform practice is an essential tool that will be used frequently." (Hamilton et al., 2009, p. 46)

Although solid evidence on a relation between data culture and student achievement is lacking, the panel (Hamilton et al., 2009) was convinced that the recommendations they provide ('establish a clear vision for schoolwide data use' and 'provide supports that foster a data-driven culture within the school') are essential, since numerous qualitative studies report that schoolwide support for data use is important. For example, in Datnow, Park, and Wohlstetter (2007) it is stated that "a culture that values the regular, consistent use of data is essential to supporting a performance-driven system" (p. 24). The (high performing) schools in their case studies found that explicit expectations and norms with regard to using data were needed in order to establish data use in the schools. Furthermore, norms and rules for discussing students were mentioned as important in order to facilitate productive discussion (Datnow et al., 2007). Additionally, a safe environment with a culture of inquiry that supports DBDM, where teachers feel empowered by data instead of threatened, is crucial (Ikemoto \& Marsh, 2007; Marsh, 2012; Schildkamp \& Poortman, 2015). This is also recognized in the case study by Park, Daly, and Guerra (2012), in which the principals in the district they studied acknowledged the need to tackle fears and overcome resistance.

It is remarkable that when it comes to culture, the first step of DBDM is primarily emphasized in the literature. However, as Ingram, Louis, and Schroeder (2004) argue, fostering data use is not the only aspect of school culture that is essential in order to implement and sustain DBDM: schools' achievement orientation is crucial as well. As is also known from school effectiveness literature (e.g. Scheerens \& Bosker, 1997), schools' achievement orientation is about fostering high expectations, a clear focus on the mastery of basic subjects, and the use of student achievement progress in order to set ambitious but attainable goals (Scheerens \& Bosker, 1997). This aligns with the first and second component of DBDM (as depicted in Figure 1): evaluating and analyzing results, and setting SMART and challenging goals.

We therefore characterize schools in which the use of data is valued and promoted, 
in which high expectations are fostered, and in which school leaders and teachers show a clear focus on the mastery of basic subjects as schools with a strong data culture.

\section{Data Culture and School Leadership}

When the establishment of such a data culture is discussed, the role of the school leader is often emphasized. Gerzon (2015) for example, stated that: "In both research and professional development practice, it is clear that leadership to support a culture of data use is essential" (p. 1). Next to initiating the improvement process and fulfilling practical preconditions, school leaders are assumed to play an important role in the successful implementation of systematical DBDM in their school by determining the vision, and influencing motivation and learning (Ikemoto \& Marsh, 2007; Levin \& Datnow, 2012; Schildkamp \& Lai, 2013). Coburn and Turner (2011) also stress that school leaders can influence the data culture of schools, by promoting a vision and norms with regard to data use.

Wayman, Cho, Jimerson and Spikes (2012), found that educators in schools with principals who facilitated data use showed positive attitudes and used data to support educational practice. According to Hallinger and Heck (2011), school leaders are responsible for shaping a coherent focus on learning in schools, and Gerzon (2015) recommended school leaders to recognize and model data use for informing instruction, to provide time and resources, to ensure a safe environment for teachers where they are supported in collaborative inquiry, and to establish and communicate a common interpretation and orientation toward data use. This is in line with Park et al. (2012), who described that school leaders need to build a culture of data use for continuous improvement. Successful school leaders in their exploratory case study did this by encouraging staff to believe in the relevance of data for improving teaching and learning, beyond accountability compliance, towards commitment for students, and by making sure teachers felt safe to use data (Park et al., 2012). Also Copland, Knapp and Swinnterton (2009) stressed that a data culture can be enhanced by a clear vision and shared understanding of the value of data use.

Although school leaders are considered to be relevant actors in establishing a data culture within their school, the practices and recommendations as described above are mainly based on anecdotal information, experiences of professional development providers, and descriptive case studies. Empirical evidence with regard to this relationship is limited and should be studied in more detail.

\section{The DBDM Intervention}

Based on the previous, it can be concluded that data culture is important for the successful implementation of DBDM in schools. Furthermore, it is expected that school leaders play a crucial role in establishing such a culture.

In the Netherlands primary schools are encouraged to use data to inform their decisions. However, this is not common practice yet (Inspectorate, 2015). Professional development in data use is regarded necessary for the implementation of DBDM (Desimone, 2009; Van Veen et al., 2011). Therefore, at the University of Twente a schoolwide DBDM intervention was developed, which was aimed at implementing and sustaining DBDM in the school, including the development of schools' data culture.

A very important intervention characteristic is the participation of the entire school team: all team members (school leader, academic coach, teachers and teacher assistants) participated in the intervention, and as a result DBDM was a shared responsibility of all team members from the start of the implementation. Furthermore, the intervention lasted two 
subsequent school years, since it is known that duration is important in order to implement and sustain schoolwide reform (Desimone, 2009). The intervention included seven meetings in the first intervention year, and five meetings in the second year.

The content of the intervention was aimed at developing a shared language for and understanding of data, achievement, and goals. The first intervention meeting was dedicated to learning how to analyze and evaluate student achievement, gaining insights in tests and test scores, and in how to interpret output from the student monitoring system. This way, a common knowledge base with regard to testing and assessment was built. Schools were stimulated to set ambitious goals for all students, based on evidence on student achievement and progress. Furthermore, team meetings were organized twice a year in which student achievement and goals were discussed, and where it was stressed that data should be used to inform practice, not to blame.

School leaders were explicitly supported in being or becoming educational DBDMleaders, for example by means of meetings with the project trainer to prepare school team meetings in which student achievement in the school was evaluated, and educational strategies were discussed.

\section{Research Questions}

A culture of data use is regarded essential for the implementation of DBDM in schools. Therefore, the intervention was aimed at both implementing and sustaining DBDM, including developing or sustaining a data culture within the participating schools. Our main research question was: how does schools' data culture develop during the intervention? More specifically, we expected data culture to remain stable in schools in which this was already established, and to improve in schools in which data culture initially was low. Furthermore, since school leaders are considered important actors in establishing a data culture within their school we were also interested in the relationship between data culture and leadership for DBDM. Finally, we checked whether school background variables such as SES, school size or urbanization, were related to a schools' data culture.

\section{Methodology}

\section{Sample}

Data on schools' data culture was measured by means of teacher perceptions. A total of 101 schools participated in the intervention, but due to planning issues, two of those schools followed an adjusted version of the intervention which did not cover two complete school years. Questionnaires were administered to all school team members in the remaining 99 primary schools at the start of the intervention, after one year, and after two years of implementing the intervention. After removing measurements for schools with fewer than five respondents at that measurement occasion, 96 unique schools remained, with on average 16 respondents per school per occasion (range 5-36) who completed the questionnaires. Respondents were mostly female (overall $80 \%$ ) and teacher (78\%). The majority had followed higher education (63\%), or had an university master's degree (22\%). Participating schools were mostly medium-sized (150 to 300 students), medium-SES, and located in suburban areas. School characteristics are presented in Table 1. 
Table 1. Sample characteristics of schools $(N=96)$

\begin{tabular}{llcc}
\hline & & $\mathbf{N}$ & $\mathbf{( \% )}$ \\
\hline School size & Small $(<150)$ & 28 & $(29.2 \%)$ \\
& Medium (150-350) & 49 & $(51.0 \%)$ \\
School-SES & Large $(>350)$ & 19 & $(19.8 \%)$ \\
& & & \\
& High & 25 & $(26.0 \%)$ \\
Urbanization & Medium & 49 & $(51.0 \%)$ \\
& Low & 22 & $(22.9 \%)$ \\
& Rural & & \\
& Suburban & 38 & $(39.6 \%)$ \\
& Urban & 40 & $(41.7 \%)$ \\
\hline
\end{tabular}

\section{Instruments and Data Collection}

The data culture questionnaire was constructed based on the achievement-orientation questionnaire by Scheerens and Bosker (1997), and also included questions specifically related to the use of student achievement data. This questionnaire included 10 items, such as "In our school, it is regularly stressed that it is important to set ambitious goals for all students", "We think we can reach ambitious goals for all students, taking their capabilities into account", and "We adapt our education for each student, based on their achievement" (see Appendix E for a translation of the entire questionnaire). All items were scored on a four-point Likert-scale, ranging from 'totally disagree' to 'totally agree'. Data on leadership for DBDM was collected by means of a teacher questionnaire as well (see: chapter 6), which included 13 items and led to the identification of five ordered classes of leadership for DBDM. Furthermore, respondents were asked for their demographic data, such as gender, age, and educational background. School background characteristics were retrieved from the database of the Inspectorate of Education.

Data collection took place in the context of broader data collection for the evaluation of intervention effects. The items were part of a larger questionnaire, and were administered at the beginning of the first intervention meeting (T1), the end of the last intervention meeting of the first year (T2), and the end of the last intervention meeting of the second year (T3). Questionnaires were administered to all intervention participants: school leaders, academic coaches, teachers and teacher assistants, except for the school leader questionnaire, which was not administered to school leaders themselves.

\section{Data Analysis}

Data culture is considered a latent school level variable, since it cannot be observed directly. It was measured by means of perceptions of teachers who are nested within the schools. A multilevel approach is therefore needed. Traditionally, these kinds of measures are calculated by aggregating individual-level responses to the school level, using the group mean as an outcome. This violates assumptions with regard to independent errors, and might increase statistical power artificially, because the sample size is not corrected for dependencies among individuals within groups. Multilevel latent class analysis can be applied to overcome these problems (Bennink, Croon, \& Vermunt, 2013; Bijmolt, Paas, \& Vermunt, 2004; Vermunt, 2003). 


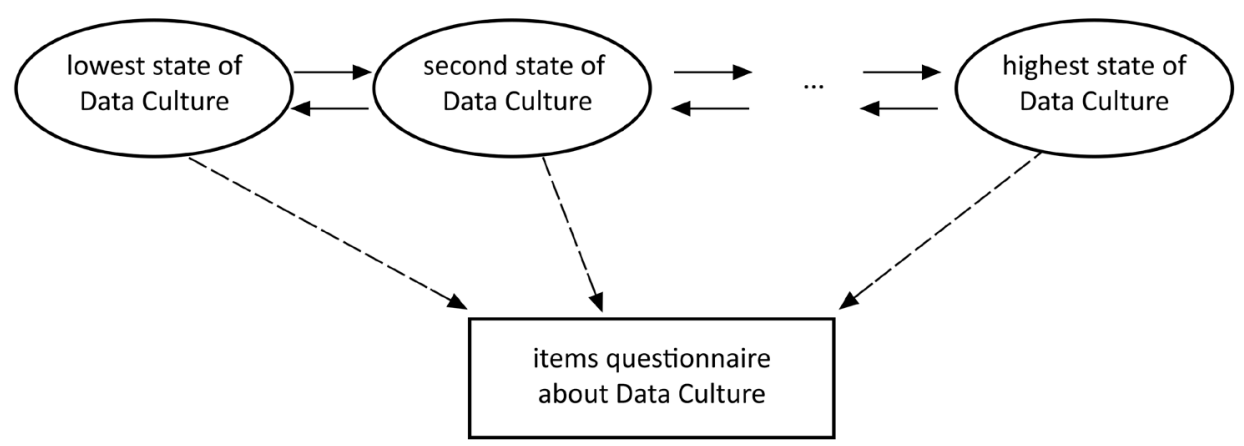

Figure 2. Proposed multi-state model for latent classes of data culture

In order to model the process of schools' movement through (ordered) latent classes of data culture, a multi-state modelling (MSM) approach is used. By means of MSM, measurement of data culture in a series of states ${ }^{1}$ can be modelled over time, and transition probabilities from state to state can be estimated (Jackson, 2014). In Figure 2, the proposed multi-state model for the latent variable 'data culture' is depicted. Dashed arrows represent the relationship of a latent class measurement model and straight arrows represent the possible transitions from one state to the next.

\section{Procedure for Data Analysis}

Step 1: Identify multilevel latent class model. The questionnaire consisted of 10 items, which were scored on a four-point Likert scale. In order to decrease the number of thresholds to be estimated, the answer categories 'totally disagree' and 'disagree' were recoded into one category, since there was only a small number of observations in the 'totally disagree' category (499 out of 44,370 answers; $1.1 \%$ ).

To identify differences in data culture across schools and time, as perceived by the school staff, multilevel latent class modelling was applied. Model estimates were obtained for a range of individual classes $(I=1, \ldots, 5)$ and school classes $(S=1, \ldots, 5)$, where the maximum number was limited to five. For each combination of $I$ and $S$, we used 400 random sets of starting values and 40 optimizations for the final model. The best solution for each combination was stored, and information criteria $(\mathrm{BIC}(K))$ for all combinations of $I$ and $S$ were compared to select the optimal number of classes at individual and school level. Analyses were performed in Mplus 7 (Muthén \& Muthén, 2004).

Although it is not always necessary or applicable when conducting LCA, we aimed at ranking the school classes in order to be able to investigate changes in and improvement of data culture during the intervention.

Step 2: Analyze initial class assignment. In order to analyze the association between initial class membership and school characteristics, and initial class membership and leadership for DBDM, Pearson's chi-square test was performed. In case of significant differences, pairwise comparisons were made by applying Fisher's exact test.

$1 \quad$ Where the latent measurements are called 'classes' in latent class analysis, these classes are labeled 'states' in multi-state modelling. These terms can therefore be used interchangeably in this paper. 
Step 3: Multi-state modelling. After identifying the optimal ML LCA model and labelling the latent classes, transitions were investigated by means of multi-state modelling (Jackson, 2011). Data culture was measured at the beginning of the intervention, at the end of the first intervention year (ten months later), and at the end of the second intervention year (22 months after the first measurement occasion). We only measured data culture states at those occasions. When a change occurred, we do not know when this exactly happened besides 'somewhere during this interval'. We used a multi-state model (MSM) to model the transitions in continuous time, applying a homogeneous continuous-time Markov model (Jackson, 2011). We were specifically interested in development, and therefore ordered the different data culture states at school level as increasing levels of data culture (DC). It is reasonable to expect that schools evolve from one state to another, without 'skipping' states in between. We therefore specified a so-called q-matrix that only allowed for transitions to adjacent states. This for example means that a school had to move through state 2 in order to change from class 1 to 3 . The model allows us to compute transition probabilities for every point in time, indicating the probability that a school which was observed as a member of class $X$, would be a member of class $X, Y$, or $Z$ after a specified number of months.

A similar procedure was followed for the analysis of transitions in leadership for DBDM in chapter 6. At school level, the measured state of leadership for DBDM was used to represent leadership for DBDM variable in this paper.

\section{Results}

In the following section, the results of the subsequent steps of the analysis are presented. First, the ML LCA model was selected. Second, the classes at individual level were interpreted and labelled. Next, the data culture classes at school level were ordered. At school level, the relationship between initial class membership and covariates was studied. Finally, a multistate model was applied to investigate transitions.

\section{LCA - Model Selection}

To identify differences in data culture across schools and time, as perceived by school staff, first multilevel latent class analysis was applied. For all combinations of individual classes $(I=1, \ldots, 5)$ and school classes $(S=1, \ldots, 5)$, information criterion $(\mathrm{BIC}(K))$ was obtained, which is presented in Table 2. Based on these information criteria, the model with five classes at school level and five classes at individual level was selected as the optimal model.

Table 2. Model fit $(B I C(K))$ for combinations of numbers of classes for schools' data culture at school and individual level.

\begin{tabular}{|c|c|c|c|c|}
\hline \multirow[b]{2}{*}{ Individual } & \multicolumn{4}{|c|}{ School } \\
\hline & 2 & 3 & 4 & 5 \\
\hline 2 & 70349,73275 & 70324,86479 & 70326,44282 & 70336,86086 \\
\hline 3 & 68941,56914 & 68782,98519 & 68767,40724 & 68768,5353 \\
\hline 4 & 68205,28152 & 68038,86159 & 67976,65366 & 67971,67973 \\
\hline 5 & $\underline{67771,76191}$ & $\underline{67534,94805}$ & $\underline{67462,68808}$ & $\underline{67458,60817}$ \\
\hline
\end{tabular}

Note. Lowest $\mathrm{BIC}(K)$ within each row is printed italic, and within each column underlined. Lowest $\mathrm{BIC}(K)$ overall is printed bold.

Class membership at individual level and at school level was determined by selecting 
the class with the largest class assignment probability. On average, at individual level the classification probability was around .86 (range: .34-1.00, SD =.16, $N=4437$ ). At school level, the classification probability was on average .85 (range: $.45-1.00, S D=.14, N=277$ ).

\section{LCA - Interpretation and Ordering of Latent States}

At the individual level, classes can be interpreted according to the associated response patterns. In Figure 3, the average response per item is shown per assigned class. Recall that the four-point Likert scale was recoded into a three-point scale by recoding the answer categories 'totally disagree' and 'disagree' into one category. Therefore, the average scores ranged from 0 to 2 .

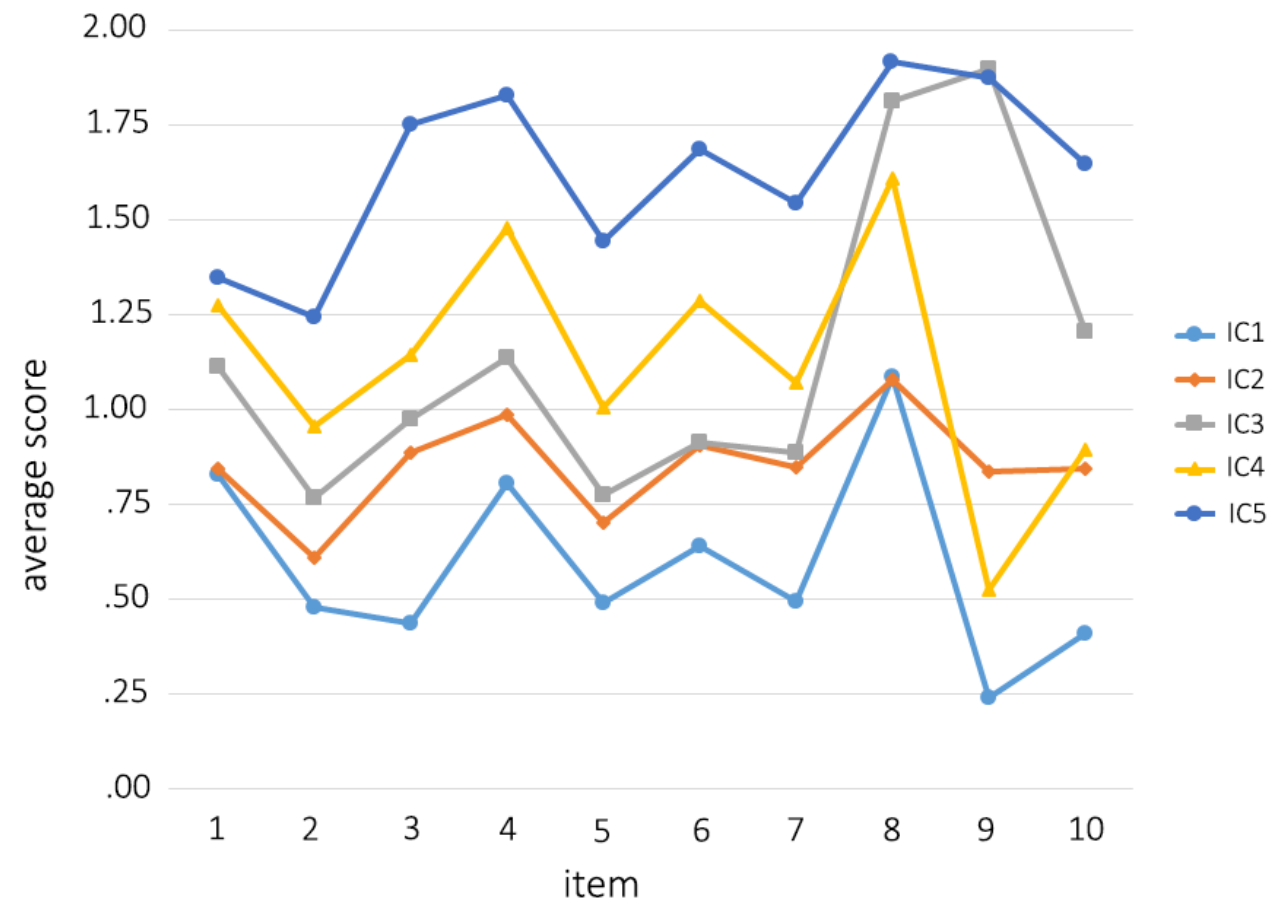

Figure 3. Average respondent item scores for individual class (IC) membership

For items 1-7, the patterns are quite comparable and only differ in height across classes, patterns for item 8, 9 and 10 are different. When taking a closer look at the content of the questions, we can argue that questions 1-7 are more about schools' achievement orientation, and questions 8,9 and 10 are more specifically related to student monitoring system use. Therefore, we ordered the individual classes based on scoring patterns for both parts of the test: the achievement oriented (AO) part (question 1-7) and the student monitoring system (SMS) part (question 8-10), see Table 3. As can be seen in Figure 3, for items 1 to 7 about achievement orientation, the lines representing the average score per individual classes show parallel patterns and can therefore be ordered accordingly. For the items with regard to the student monitoring system (item 8-10) this is less straightforward. Individual class IC3 and IC5 clearly have highest scores for these items, whereas scores for IC3 are noticeably lowest. Average respondent scores in IC2 are all medium for these items. In IC4 a high score 
is observed on item 8, whereas a low score is observed on item 9 and 10 . Therefore, IC4 is also labelled 'medium' for SMS-related items.

Table 3. Categorization of individual classes (IC) based on scores for AO and SMS-related items

\begin{tabular}{lll}
\hline IC & AO & SMS \\
\hline 1 & Low & Low \\
2 & Medium & Medium \\
3 & Medium & High \\
4 & High & Medium \\
5 & High & High \\
\hline
\end{tabular}

Table 4. Proportion respondents per IC per DC (Data Culture class)

\begin{tabular}{lccccc}
\hline $\mathbf{D C}$ & $\mathbf{I C 1}$ & $\mathbf{I C 2}$ & $\mathbf{I C 3}$ & IC4 & IC5 \\
\hline 1 & $\mathbf{8 8 \%}$ & $1 \%$ & $0 \%$ & $11 \%$ & $1 \%$ \\
2 & $\mathbf{3 9 \%}$ & $\mathbf{3 6 \%}$ & $5 \%$ & $18 \%$ & $1 \%$ \\
3 & $10 \%$ & $\mathbf{4 8 \%}$ & $16 \%$ & $20 \%$ & $6 \%$ \\
4 & $2 \%$ & $\mathbf{3 2} \%$ & $\mathbf{5 2 \%}$ & $2 \%$ & $13 \%$ \\
5 & $0 \%$ & $23 \%$ & $13 \%$ & $1 \%$ & $\mathbf{6 3 \%}$ \\
\hline
\end{tabular}

In Table 4, the proportion of respondents per individual class are presented for each data culture state. In order to determine the ranking of data culture states, for each school class we used the most common individual class (\#1 IC). In case there was another individual class to which more than $25 \%$ of respondents was assigned to, we combined this with the scores for this class (\#2 IC). See Table 5 for an overview of how the various data culture classes were interpreted. These classes were regarded as subsequent states of data culture.

Table 5. Interpretation of schools' data culture classes

\begin{tabular}{llll}
\hline DC & \#1 IC & \#2 IC & Data culture \\
\hline 1 & $1(88 \%)$ & & Really low \\
2 & $1(39 \%)$ & $2(36 \%)$ & Low to medium \\
3 & $2(48 \%)$ & & Medium \\
4 & $3(52 \%)$ & $2(32 \%)$ & Medium to high \\
5 & $5(63 \%)$ & & High \\
\hline
\end{tabular}

\section{Covariates in Relation to Initial Class Membership}

By means of chi-square tests, we investigated whether there was an association between initial data culture and school size, SES, and urbanization. For none of these background variables, a significant effect was found.

Because school leaders are regarded as essential for the implementation of DBDM we expected that schools with stronger leadership for DBDM would also have a stronger data culture. School leadership for DBDM was also measured by means of ML LCA (see: chapter 6). Just as for the data culture classes, higher class numbers indicate stronger leadership for DBDM in the school.

In Table 6, the number of schools initially assigned to each data culture class, for each class of leadership for DBDM is presented. The results in Table 6 suggest that the stronger the leadership for DBDM, the stronger the data culture, since in schools with a school leader who did not show leadership for DBDM, data culture was regarded weak as well. Chi-square 
tests also revealed a significant $(p<.05)$ difference for SL1 and SL2 compared to SL5, and for SL1 compared to SL3.

Table 6. Measures states of leadership (school level) for each measured state of data culture at the first occasion

\begin{tabular}{lccccc}
\hline $\begin{array}{l}\text { Initial data } \\
\text { culture class }\end{array}$ & SL1 & SL2 & $\frac{\text { Leadership for DBDM }}{2}$ & SL3 & SL5 \\
\hline DC1 & 9 & 11 & 4 & 4 & 0 \\
DC2 & 4 & 15 & 11 & 4 & 2 \\
DC3 & 0 & 8 & 3 & 2 & 2 \\
DC4 & 0 & 3 & 4 & 1 & 4 \\
DC5 & 0 & 0 & 0 & 0 & 1 \\
\hline
\end{tabular}

\section{Modelling Transitions - Multi-State Model}

In Table 7, for each of the measurement occasions, the number of schools assigned to each data culture class is presented. There proves to be variation in class memberships at each measurement occasion, indicating differences between schools and between occasions.

Table 7. Class memberships at each measurement occasion

\begin{tabular}{lccccc}
\hline & & & Data culture class & & \\
\cline { 5 - 6 } Occasion & DC1 & DC2 & DC3 & DC4 & DC5 \\
\hline Start & 28 & 37 & 15 & 13 & 1 \\
After one year & 4 & 2 & 27 & 27 & 4 \\
After two years & 0 & 12 & 33 & 41 & 7 \\
\hline
\end{tabular}

The estimated transition probabilities after 22 months are presented in Table 8, indicating the probability of being a member of each of the school data culture classes at the end of the intervention, given the initial class membership. The transition probabilities suggest that it is very unlikely to be assigned to class DC1 at the end of the intervention: schools with initial low data culture (member of DC1) have a .99 probability to be a member of a higher class (mostly DC3 or DC4) at the end of the intervention. Also for schools with an initial low to medium (DC2), or a medium (DC3) data culture are inclined to improve. For schools initially assigned to DC4, stability is most likely. It is remarkable that a decrease to DC4 has the largest probability for schools that were initially member of the highest data culture class. However, note that there was only one school assigned to DC5 at the first measurement occasion.

Table 8. Probability of being assigned to a data culture class at the end of the intervention, given the initially assigned class

\begin{tabular}{lccccc}
\hline \multirow{2}{*}{ Initial class } & DC1 & DC2 & DC3 & DC4 & DC5 \\
\hline DC1 & .01 & .23 & .40 & .32 & .04 \\
DC2 & .00 & .14 & .38 & .42 & .05 \\
DC3 & .00 & .02 & .28 & .59 & .10 \\
DC4 & .00 & .00 & .09 & .72 & .18 \\
DC5 & .00 & .00 & .05 & .56 & .39 \\
\hline
\end{tabular}




\section{Conclusions and Discussion}

Data culture is regarded essential for the successful implementation of DBDM in schools. In the current study, the data culture of schools participating in an intensive intervention aimed at implementing and sustaining DBDM was measured and studied before the start of the intervention, as well as during and after the intervention.

Because of the importance of both analyzing data as well as of using these data in order to set ambitious goals, we, in the operationalization of data culture included student monitoring system use and schools' achievement orientation. This 'expanded definition' of data culture better fits the definition of DBDM in general, and proved to be a suitable operationalization in practice. Based on the analyses of the individual class membership and answering patterns at the questionnaire, it can be concluded that both components are important elements for school's data culture: the majority of individuals in schools belonging to the lowest data culture class had low scores on achievement orientation, and all showed low scores on the questions related to SMS use. All individuals in schools of DC5 scored high on SMS questions, and high, or medium, on the questions about the school's achievement orientation.

\section{Main Findings}

Our main research question was: how does schools' data culture develop during the intervention? More specifically, we expected data culture to remain stable in schools in which such a culture had already been established, and to improve in schools in which data culture initially was low. It appeared that schools in which the data culture could be classified as 'low' at the start of the intervention, evolved to a 'medium' data culture, and that for schools with an initial 'medium' data culture a transition to 'high' was likely. For schools with an initial high data culture, stability was most likely, indicating that schools in which the data culture had already been established indeed were able to maintain this level of data culture. These findings indicate that schools' data culture, which is considered an important factor for the implementation of DBDM, can be developed during an intensive intervention.

Furthermore, we were interested in the relationship between school leadership and DBDM, since school leaders are regarded important in establishing a data culture within their school. Until now, empirical evidence with regard to this relationship was lacking. In the current study, an empirical association between school leadership for DBDM and data culture was found: data culture was higher in schools in which school leaders were classified as more DBDM oriented. Although several authors (e.g. Coburn \& Turner, 2011; Ikemoto \& Marsh, 2007; Levin \& Datnow, 2012; Schildkamp \& Lai, 2013) suggest that school leadership for DBDM is important for schools' data culture, to our knowledge, this is the first study in which this relationship was demonstrated.

\section{Limitations}

The first limitation with regard to this study is related to the instrument in order to determine data culture in the participating schools, which consisted of only ten items. In order to more thoroughly investigate data culture, a more elaborate instrument could provide even more insight into different areas of data culture, and into patterns of development. However, since this questionnaire was part of a larger data collection, it was not feasible to include more items - completing the questionnaire would then have taken too long, with a high risk of loss of respondents. However, we recommend the development of a broader, more 
comprehensive instrument in order to measure data culture.

Another limitation is related to the sample in the current study. All schools participated in the intervention, which could for example be an explanation for the large number of schools assigned to low classes of data culture prior to the intervention: these schools may have applied for participating in the intervention because of the low level of data culture in the school.

\section{Further Research}

In the current study, it was shown that data culture can be enhanced during an intervention aimed at implementing and sustaining DBDM in schools, and that schools with an initial high data culture were able to maintain this level of data culture. However, since all schools in this study participated in the intervention, for future studies it is recommended to include a control group in order to gain insight into general patterns of change in data culture, and to investigate whether improvement can be attributed to participating in the intervention.

Finally, we were not able to relate data culture to the degree of implementation of DBDM, or the improvement of student achievement yet. In a follow-up study, the relationship between data culture and improved student achievement during the DBDM intervention will be investigated. 


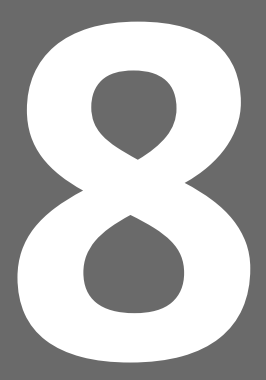

\section{The Transformation of Schools' Social Networks \\ During a Data-Based Decision Making Reform}

This chapter is a modified version of the article:

Keuning, T., Van Geel, M., Visscher, A.J., Fox, J.-P., \& Moolenaar, N. (accepted). The transformation of schools' social networks during a databased decision making reform. Teachers College Record 


\begin{abstract}
Background. Collaboration within school teams is considered to be important to build the capacity school teams need, to work in a data-based way. In a school characterized by a strong collaborative culture, teachers may have more access to the knowledge and skills for analyzing data, teachers have more opportunity to discuss the performance goals to be set, and they also can share effective teaching strategies to achieve those goals. Although many studies on data-based decision making (DBDM) foreground the importance of teacher collaboration, our knowledge on what such collaboration looks like and how such collaboration may change during a DBDM reform, remains limited.
\end{abstract}

Purpose. The current study uses a social network perspective to explore how collaboration in 32 elementary schools in the Netherlands takes shape in the interactions among teachers as they engage in a DBDM reform project.

Method. Schools' social networks were examined at the start of the intervention and after having participated one year in the DBDM reform. Social networks regarding three DBDM topics are examined: (1) discussing student achievement; (2) discussing the achievement goals; (3) and discussing instructional strategies. The density, reciprocity and centralization of these networks were calculated, and multivariate multiple regression analysis was used to analyze changes over time.

Findings. Findings suggest that teachers' DBDM related networks transform during the intervention, especially regarding the discussion of student achievement data: although the number of relationships remains stable, more reciprocal relationships are formed, and this network becomes less centralized around one, or a few influential staff members. 
Around the world, there is growing emphasis on data use in education (Mandinach \& Gummer, 2015). In the international literature, the use of data to improve teaching and learning is frequently referred to as Data-Based Decision Making (DBDM). Ikemoto and Marsh (2007) referred to DBDM as "teachers, principals, and administrators systematically collecting and analyzing data to guide a range of decisions to help improve the success of students and schools" (p. 108). Teachers' use of student achievement data to evaluate student progress, to provide tailor-made instruction, and to develop strategies for maximizing performance is considered to have a positive influence on student outcomes (Schildkamp, Ehren, \& Lai, 2012).

An important factor to support DBDM is a collaborative school culture (Ingram, Louis, \& Schroeder, 2004; Lai \& Schildkamp, 2013; Schildkamp \& Poortman, 2015). In a school characterized by a strong collaborative culture, teachers may have access to the knowledge and skills for analyzing data; have the opportunity to discuss challenging performance goals; and share effective teaching strategies to achieve those goals (Ingram et al., 2004; Lai \& McNaughton, 2009; Lai \& Schildkamp, 2013). Strong collaboration thus can be considered a precondition for DBDM, and therefore reform initiatives aimed at improving DBDM should encourage collaboration. Although studies on DBDM foreground the importance of teacher collaboration, our knowledge on what an optimal collaboration structure for DBDM looks like, and whether DBDM professional development can support such collaboration is very limited. Therefore, the current study took a social network perspective to explore how collaboration takes shape in the interactions among teachers as they engage in a DBDM reform project. The change of the social networks at 32 elementary schools in the Netherlands was examined as they participated in a two-year DBDM professional development reform.

\section{Theoretical Framework}

In the following section, first DBDM is defined and it is explained why collaboration is considered important for the successful implementation of a school reform like DBDM. Second, we explain how the social network perspective might help us understand the collaboration patterns within schools, and we elaborate on what already is known about social network transformation during a reform initiative. Third, a brief description of the DBDM intervention studied in this paper is given. Finally, the hypotheses will be stated.

\section{Data-Based Decision Making and the Importance of Collaboration}

Systematically analyzing student performance data, setting goals based on such data and trying to accomplish maximum achievement for all students in the school is referred to as Data-Based Decision Making. In the context of this study, DBDM was decomposed into four components at three levels, graphically represented in Figure 1, based on Visscher and Ehren (2011). 


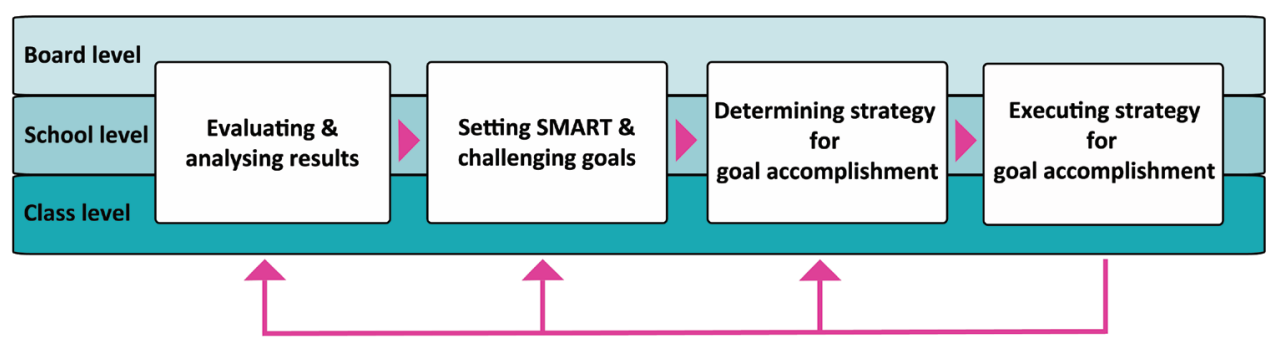

Figure 1. The components of DBDM at three levels

At the class, school and board levels, student and school performance data is intended to be first analyzed, and decisions are intended to be made based on such data. The term 'data' is relatively broad and can include several types of data to base decisions on (Schildkamp \& Poortman, 2015). In this study, the primary focus was on standardized test results comparable to what is called interim benchmark assessments by Datnow and Hubbard (2015). In the Dutch context, a student monitoring system has been developed by the Central Institute of Test Development (Kamphuis \& Moelands, 2000). This system includes a coherent set of tests for the longitudinal assessment of students' achievement throughout all grades of primary education. These tests, which are usually taken twice a year (in January and July), are available for all core subjects (mathematics, reading, spelling, vocabulary). The test results are converted into an ability scale for each subject, so student progress can be monitored over grades and school years (Kamphuis \& Moelands, 2000). Furthermore, the accompanying student monitoring software enables teachers to monitor their students' strengths and weaknesses on the basis of these assessment results, and to adapt their instruction accordingly.

The analysis of the assessment results is the starting point, but additional information, such as curriculum-based tests, classwork, homework and classroom observations, also needs to be included because no single assessment provides all information to make informed decisions (Hamilton et al., 2009).

In the DBDM literature, the term 'decisions' implies a variety of actions that can be undertaken based on all such data, such as setting goals, adapting instruction, adapting the curriculum, evaluating the effectiveness of programs and practices, and reallocating time and resources as necessary (Earl \& Katz, 2006; Hamilton et al., 2009; Ikemoto \& Marsh, 2007; Mandinach, Gummer, \& Muller, 2011). In Figure 1, 'decisions' are decomposed into setting goals based on the available data, and determining the strategies that are intended to achieve those goals.

The chosen goals and strategies for goal accomplishment vary according to the level of decision-making. At the group level, teachers may use student performance data to set goals in terms of the desired achievement gain, or skill attainment. To accomplish those goals, they may decide to use a specific instructional strategy, or form an ability group to work on a specific topic (Dunn, Airola, Lo, \& Garrison, 2013). At the school and school board levels, data can be used to highlight specific areas for improvement in the school(s). The strategies chosen often comprise policy decisions, for example regarding the allocation of resources, the adoption of instructional strategies throughout the entire school, or the modification of the curriculum.

The final step in Figure 1 is to implement and execute the chosen strategies. As 
Bennett (2011) and Anderson, Leithwood, and Strauss (2010) argued, the effects of the implementation activities are closely related to the quality of the inferences based on the data, the chosen approach to address the identified problems, and the expertise of school staff. If teachers, for example, draw invalid conclusions about students' learning needs, they are likely to implement a strategy that may not lead to the desired outcomes. Such invalid conclusions may be caused by a lack of knowledge on what the data say, but also by the quality of the test (Cronbach \& Meehl, 1955) used for measuring student achievement. The quality of the test is among other things influenced by the extent to which the items in that test, as a sample of items from the universe of possible items, represent that universe well and as such provide a valid picture of how well a student masters specific subject matter content (Cronbach \& Meehl, 1955).

Teachers' use of student achievement data for evaluating student progress, for providing tailor-made instruction, and for developing strategies for maximizing performance is considered to have a positive influence on student outcomes. However, the effectiveness of DBDM will strongly depend on the extent to which teachers possess or develop the required professional knowledge and skills for analyzing and interpreting the performance feedback, and for translating performance data into improvement strategies (Schildkamp \& Poortman, 2015). Collaboration within school teams is important to build the capacity school teams need to work in a data-based way (Farley-Ripple \& Buttram, 2015; Marsh, Bertrand, \& Huguet, 2015). In a highly collaborative team, teachers might have more access to the knowledge and skills for analyzing data; have the opportunity to discuss challenging goals; and share effective teaching strategies to reach these goals (Farley-Ripple \& Buttram, 2015; Jimerson \& Wayman, 2015). A collaborative school culture is assumed to have a positive effect on the implementation of educational reforms such as DBDM (Ingram et al., 2004; Lai \& McNaughton, 2009; Love, Stiles, Mundry, \& DiRanna, 2008; Schildkamp \& Lai, 2013). Schools with strong collaboration patterns seem to facilitate the transfer of complex information, and show improved individual and organizational performance. These schools are more characterized by the trust necessary for risk taking during reform. Teachers working in collaborative teams also seem to be more likely to improve their instructional practices, and to have more access to expertise and support (Daly, Moolenaar, Bolivar, \& Burke, 2010; Penuel, Sun, Frank, \& Gallagher, 2012). Additionally, in schools characterized by strong relationships, the innovative climate is stronger (Moolenaar et al., 2014; Moolenaar, Daly, \& Sleegers, 2010), indicating that there is a higher "collective willingness to adopt an open orientation toward new practices and change, and to collectively support and develop new knowledge, practices and refinements to meet organizational goals" (Moolenaar et al., 2014, p. 102).

More specifically, in their study of DBDM in high schools, Ingram et al. (2004) concluded that "teachers are more likely to mention systematic data collection and use when they are involved in groups looking at school processes." (p. 1280). Schildkamp and Lai (2013) stated that collaboration is essential for sustainable school improvement: collaboration reduces the isolation of the teaching profession and improves individual teachers' personal growth.

\section{Social Network Characteristics for School Reform}

What is the 'optimal' DBDM social network? Both in the educational literature (Daly et al., 2010; Finnigan \& Daly, 2012; Frank, Penuel, \& Krause, 2015; Sun, Penuel, Frank, Gallagher, \& Youngs, 2013) as well as in the literature on organization and management 
(Borgatti \& Foster, 2003; Devine, Clayton, Philips, Dunford, \& Melner, 1999; Sparrowe, Liden, Wayne, \& Kraimer, 2001; Tsai, 2001) social networks are associated with team learning and organizational learning and performance. The success of the implementation of an innovation, such as DBDM, depends critically on the ability of the organization to internally distribute the knowledge, support and resources necessary to promote practices related to this innovation (Frank et al., 2015).

Although studies on DBDM stressed the importance of teacher collaboration, our knowledge on what such collaboration looks like is still limited. The social network perspective might give us more insight into collaboration patterns that enhance the implementation of DBDM in schools (Daly, 2012).

Social network analysis is a systematic approach used to quantify and visualize the patterns of relationships (called 'ties') between actors (e.g. teachers) in a social network (e.g., a school) (Moolenaar, 2010). In this study we focused on three network measures that are generally used to define schools' network structure: density, centralization and reciprocity (Moolenaar, 2012). Density refers to the concentration of relationships in a social network and is calculated as the total number of relationships in the network divided by the total number of possible relationships (Moolenaar, 2012). Since ties are considered paths through which information and knowledge can flow, it is assumed that the more relationships exist, the more access actors have to information and knowledge within the network (Borgatti \& Ofem, 2010). Accordingly, in dense networks - with many ties - resources are moved more quickly than in networks with fewer ties (Scott, 2013). Dense networks are important for an innovative climate. Moolenaar et al. (2011) found that the more densely connected teachers were in regard to work-related and personal advice, the more they perceived their school's climate to be supportive of innovation. Moreover, Daly et al. (2010) found that school team members in a dense network during an educational reform reported being able to enact the reform at a greater depth compared to those team members who were sparsely connected to each other. Furthermore, denser networks were associated with greater focus on teaching and learning as well as increased collective action, efficacy, and collective satisfaction. Finnigan and Daly (2012) pointed to the fact that more dense networks provide a better basis for meeting collective goals, and the stable relationships within them enable cooperation, innovation and the exchange of knowledge. Daly and Finnigan (2011) however stressed that stable ties in dense networks may form a barrier for the assimilation of new external information just as for flexible organizational responses to external stimuli.

A second measurement of the compactness of a network, complementary to density is the centralization of the network (Scott, 2013). Centralization indicates the degree to which a network is centralized around one or more 'popular' actors (Moolenaar, 2012). Which degree of centralization is effective is strongly dependent on the goal of the network. More centralized networks are positively related with the exchange of simple information, technical knowledge and routines (Cummings \& Cross, 2003) while decentralized structures are more suited for solving complex group tasks (Sparrowe et al., 2001), and are more flexible towards change and innovation (Daly \& Finnigan, 2011).

Finally, the extent to which relationships in a network are reciprocal is indicated by its reciprocity (Moolenaar, 2012) which reflects the number of reciprocal relationships within a network divided by the total sum of all relationships in the network (whether reciprocal or not). Kilduff and Tsai (2003) suggested that sharing complex information and knowledge is correlated with the existing number of reciprocal relationships.

Based on the literature we assumed that social networks that support DBDM 
are characterized by many ties through which information can flow (high density), equal relationships across the network to ensure that teachers collectively participate in the DBDM process (low centralization), and a high number of reciprocal ties through which complex information and knowledge about DBDM is exchanged. These types of networks were assumed to support the exchange of complex information, such as knowledge about analyzing and interpreting data, or the exchange of effective teaching strategies.

What does DBDM collaboration look like in practice? Farley-Ripple and Buttram (2015) performed a case study on the structure of data advice networks compared to teachers' regular professional network. Findings showed that teachers' data use networks were highly similar to their 'regular professional network' in terms of which individual teachers seek support from who, but different in terms of density and centralization: professional networks were denser than the data advice network, whereas the latter was more centralized. However, as Farley-Ripple and Buttram (2015) emphasized, this case study was done in a high-performing elementary school, a prime example of a school who already worked in a DBDM way, which might not be generalizable to other schools.

In their study on schools' social networks Atteberry and Bryk (2010) found that in many schools teachers work in isolation, experience little task interdependence, and have few opportunities for professional collaboration. Teachers mainly discuss work-related issues with teachers within the same grade level, also known as the 'homophily' principle ("the principle that a contact between similar people occurs at a higher rate than between dissimilar people"), or with the teachers in the classroom next to them: the 'proximity' principle ("the physical distance separating people in the workplace, and the likelihood that they will communicate about their work") (Coburn, Choi, \& Mata, 2010). This often leads to social networks that are limited and homogeneous, and that largely focus on grade-level colleagues. When it comes to a specific topic, teachers tend to form ties with colleagues who have expertise on that specific topic (Coburn et al., 2010). Staman, Visscher and Luyten (2014) found that the academic coach and the school leader are the most knowledgeable persons regarding the interpretation of student outcome data from a student monitoring system, and with respect to the types of analyses that can be conducted using that system. In the Dutch context, the academic coach advices teachers on how to deal with students with special needs, gives instructional advice to the teacher, or tries to find external help. Based on their role within the school team, it was expected that teachers will reach out most to the academic coach for discussing student achievement, and the goals to be accomplished. This might lead to unequally divided relationships within a team, where the 'expert' within the team has most power and decides which information to share.

Since many authors (Atteberry \& Bryk, 2010; Coburn \& Russell, 2008; Finnigan \& Daly, 2012; Penuel \& Riel, 2007) stated that reform initiatives are more successful in schools that have more developed social networks, it seems that social networks, characterized by low collaboration and unequally divided relationships within the school team, will not to be a solid foundation for comprehensive school reform.

A reform initiative could influence the collaboration patterns of a school team, but only a few studies have looked into the transformation of social networks within schools, especially where such development is the result of school improvement interventions (Moolenaar, 2012). In their case studies of four elementary schools during the implementation of a new mathematics curriculum, Coburn et al. (2010) found that as a result of the intervention in which teachers were encouraged to collaborate networks became denser and more diverse. Furthermore, during reform implementation, there was a desire among teachers to interact 
with those colleagues perceived as 'having relevant expertise'. This tension to interact with experts on implementation reform was also observed in other studies (Atteberry \& Bryk, 2010; Coburn et al., 2010). However, in these studies reform was aimed at training specific staff within the team to become experts.

Problem statement. To our knowledge, studies of the effect of an intervention in which the entire team is involved in team network structures are non-existent. Furthermore, until now the analysis of social networks within schools has mainly been of a cross-sectional kind, has been based on small samples, and includes many case studies. For our better understanding of the role of social networks in school development efforts, longitudinal studies based on larger samples in which the transformations of social networks during a school wide reform are investigated are needed. Therefore, the current study took a social network perspective to explore how collaboration takes shape in the interactions among school staff as they engage in a schoolwide DBDM reform. This study was guided by the following research question: How do collaboration patterns within schools at the start of a DBDM reform look like and how do they change during that reform? In order to answer this research question, first the collaboration patterns at the start of the DBDM reform were explored, and it also was explored whether school characteristics (like the size of the team, the average age of the team members, and the SES of the students in the school) were associated with these initial collaboration structures. Next, the transformations of the social networks during the DBDM reform were studied.

\section{The DBDM Intervention}

Like a number of U.S. interventions (e.g. Boudett, City, \& Murnane, 2005; Carlson, Borman, \& Robinson, 2011; Love et al., 2008; Slavin, Cheung, Holmes, Madden, \& Chamberlain, 2012), the DBDM intervention in this study was a two-year training course for entire elementary school teams in the Netherlands, aimed at implementing and sustaining DBDM in the entire school organization, by systematically following the DBDM cycle as shown in Figure 1. For the purpose of this study we focused on collaboration within school teams before and after the first year of the intervention. In Table 1 the outline of the first intervention year is shown.

Schools chose one subject to focus on during the first intervention year: mathematics, spelling, reading, or vocabulary. During the first year training year, the four DBDM components (Figure 1) were introduced one by one. The first four meetings were primarily dedicated to acquiring the knowledge and skills for DBDM: using the student monitoring system, analyzing and interpreting test score data, diagnosing learning needs, setting goals, and developing instructional plans. Between meeting 1.4 and 1.5 teachers executed their instructional plans in their classrooms. During meeting 5 the DBDM cycle was completed for the first time when the new student achievement data were discussed during a team meeting. The trainers then stressed that these data were supposed to be used for improvement purposes, and not for judging colleagues. The goal was to develop a culture of trust and collaboration in which the school team as a whole felt the responsibility for the performance of their students. The sixth meeting was aimed at teacher peer consultation: providing teachers with the knowledge and tools for observing their colleagues in the classroom, and for providing them with feedback based on those observations. In meeting seven (like in meeting 1.5) the new student test results were again discussed with the entire school team.

Since it was assumed that teacher collaboration is important for the successful implementation of DBDM, the intervention was offered as a team training. The entire team, 
Table 1. Overview of the first year of the DBDM intervention

\begin{tabular}{|c|c|c|}
\hline & Type of meeting & Content \\
\hline & $\begin{array}{l}\text { School leader / } \\
\text { school board meeting }\end{array}$ & $\begin{array}{l}\text { Fulfilling practical preconditions and stressing the importance of } \\
\text { the role of the school leader/school board }\end{array}$ \\
\hline 1.1 & $\begin{array}{l}\text { Team meeting } \\
\text { (full day) }\end{array}$ & Analyzing test score data from the student monitoring system \\
\hline 1.2 & $\begin{array}{l}\text { Team meeting } \\
\text { (half day) }\end{array}$ & $\begin{array}{l}\text { Subject matter content - curriculum } \\
\text { Individual diagnosis of students' learning needs }\end{array}$ \\
\hline 1.3 & $\begin{array}{l}\text { Team meeting } \\
\text { (half day) }\end{array}$ & Goal setting and developing instructional plans \\
\hline 1.4 & $\begin{array}{l}\text { Team meeting } \\
\text { (half day) }\end{array}$ & $\begin{array}{l}\text { Instructional plans in practice; monitoring and adjusting } \\
\text { instructional plans based on test data from content mastery } \\
\text { tests and daily work in class }\end{array}$ \\
\hline & $\begin{array}{l}\text { School leader / } \\
\text { school board meeting }\end{array}$ & $\begin{array}{l}\text { Discussing progress and the goals for the next period (trainer, } \\
\text { school leader and school board) }\end{array}$ \\
\hline 1.5 & $\begin{array}{l}\text { Team meeting } \\
\text { (half day) }\end{array}$ & Team meeting: evaluating standardized test performance data \\
\hline 1.6 & $\begin{array}{l}\text { Team meeting } \\
\text { (half day) }\end{array}$ & $\begin{array}{l}\text { Collaboration in the school: how to learn from each other by } \\
\text { using classroom observations }\end{array}$ \\
\hline & $\begin{array}{l}\text { School leader / } \\
\text { school board meeting }\end{array}$ & $\begin{array}{l}\text { Discussing progress and goals for the next period (trainer, school } \\
\text { leader and school board) }\end{array}$ \\
\hline 1.7 & $\begin{array}{l}\text { Team meeting } \\
\text { (half day) }\end{array}$ & Team meeting: evaluating standardized test performance data \\
\hline
\end{tabular}

including school leaders, academic coaches, teachers, and sometimes also teaching assistants, participated in the project. This collective participation is in line with the professional development literature stating that the interaction and collaboration between colleagues is important when implementing and mastering a schoolwide innovation (Timperley, 2008; Van Veen, Zwart, \& Meirink, 2011). Moreover, all intervention meetings included collaborative activities, such as activities in which colleagues shared ideas, adjusted performance goals, exchanged teaching strategies with each other, and cooperatively wrote instructional plans.

School leaders and academic coaches were also coached by the trainer to improve collaboration within the team, for example, through allocating time for collaborative activities, organizing team meetings in addition to the training sessions, and encouraging teachers to ask for advice regarding teaching and classroom management strategies.

The training was provided by trainers who had been appointed by the University of Twente, and the project was supervised by the first author who was not directly involved in working with the schools. To ascertain that the training was as much as possible the same across schools and trainers, each meeting had a central topic as shown in Table 1. This topic was the same for every participating school. The content of the meetings was fixed for all schools, the same PowerPoint slides were used, and the same exercises were done in all schools. Before each meeting the trainers discussed the content for that specific meeting intensively, to assure that each of them would present the information in the same way. Because of variation in school teams' prior knowledge, team members' needs, and the 
Table 2. Sample characteristics of schools $(N=32)$ and individuals $(n=512)$

\begin{tabular}{|c|c|c|c|c|c|}
\hline \multirow{2}{*}{\multicolumn{6}{|c|}{ Individual level }} \\
\hline & & & & & \\
\hline \multirow[t]{2}{*}{ Gender } & \multicolumn{3}{|l|}{ Male } & 69 & $(13.5 \%)$ \\
\hline & \multicolumn{3}{|l|}{ Female } & 443 & $(86.5 \%)$ \\
\hline \multirow{4}{*}{$\begin{array}{l}\text { Experience in } \\
\text { elementary } \\
\text { education }\end{array}$} & \multicolumn{3}{|l|}{$0-3$ years } & 45 & $(8.8 \%)$ \\
\hline & \multicolumn{3}{|l|}{ 4-10 years } & 147 & $(28.7 \%)$ \\
\hline & \multicolumn{3}{|l|}{$>10$ years } & 318 & $(62.1 \%)$ \\
\hline & \multicolumn{3}{|l|}{ Unknown } & 2 & $(0.4 \%)$ \\
\hline \multirow[t]{7}{*}{ Role } & \multicolumn{3}{|l|}{ School leader } & 34 & $(6.6 \%)$ \\
\hline & \multicolumn{3}{|c|}{ Deputy school leader } & 5 & $(1.0 \%)$ \\
\hline & \multicolumn{3}{|l|}{ Academic coach } & 38 & $(7.4 \%)$ \\
\hline & \multicolumn{3}{|c|}{ Teacher Kindergarten grades (age 4-6) } & 100 & $(19.5 \%)$ \\
\hline & \multicolumn{3}{|c|}{ Teacher lower junior grades (age 7-9) } & 148 & $(28.9 \%)$ \\
\hline & \multicolumn{3}{|c|}{ Teacher upper junior grades (age 10-12) } & 164 & $(32.0 \%)$ \\
\hline & \multicolumn{3}{|c|}{ Other (e.g. remedial teacher, teacher assistant) } & 23 & $(4.5 \%)$ \\
\hline \multirow[t]{4}{*}{ Educational level } & \multicolumn{3}{|l|}{ University degree } & 12 & $(2.3 \%)$ \\
\hline & \multicolumn{3}{|c|}{ Higher vocational education } & 442 & $(86.3 \%)$ \\
\hline & \multicolumn{3}{|c|}{ Intermediate vocational education } & 51 & $(10.0 \%)$ \\
\hline & \multicolumn{3}{|l|}{ Other } & 7 & $(1.4 \%)$ \\
\hline \multicolumn{6}{|l|}{ School level } \\
\hline \multirow[t]{3}{*}{$\overline{\text { School size }}$} & \multicolumn{3}{|l|}{ Small $(<150)$} & 7 & $(21.9 \%)$ \\
\hline & \multicolumn{3}{|l|}{ Medium (150-350) } & 18 & $(56.3 \%)$ \\
\hline & \multicolumn{3}{|l|}{ Large $(>350)$} & 7 & $(21.9 \%)$ \\
\hline \multirow[t]{3}{*}{ School-SES } & High & & & 8 & $(25.0 \%)$ \\
\hline & Medium & & & 18 & $(56.3 \%)$ \\
\hline & Low & & & 6 & $(18.8 \%)$ \\
\hline Urbanization & Urban (4 largest cit & e Nethe & & 5 & $(15.6 \%)$ \\
\hline & Suburban (next 32 & cities in & therlands) & 13 & $(40.6 \%)$ \\
\hline & Rural (smaller tow & illages) & & 14 & $(43.8 \%)$ \\
\hline & $\mathbf{N}$ & $M$ & SD & Min & Max \\
\hline Individual level & & & & & \\
\hline Age staff & 512 & 43.9 & 12.0 & 22 & 64 \\
\hline School level & & & & & \\
\hline Gender ratio & 32 & 86.5 & 7.0 & 72.7 & 100.0 \\
\hline Average age staff & 32 & 44.0 & 4.3 & 35.5 & 53.9 \\
\hline School size (students) & 32 & 248.3 & 112.9 & 69 & 545 \\
\hline Team size & 32 & 16.0 & 5.7 & 8 & 29 \\
\hline
\end{tabular}


subject chosen by a school, the time a trainer spent on a specific topic within a meeting varied somewhat over schools.

Although the content of meetings was very similar across schools, what schools after the meetings did with the information received varied and was influenced by several factors such as the characteristics of the school leader of the school, teachers' attitudes towards the intervention, and the time that could be spent on DBDM.

\section{Hypotheses}

Since DBDM comprises several components, three social networks related to DBDM were studied: the social network for discussing student achievement, the network for discussing the performance goals that teachers would like to accomplish, and finally the network for discussing instructional strategies. Since the intervention encouraged school teams to discuss these DBDM issues, and consequently aimed at improving the relationships regarding DBDM, it was expected that during the course of the intervention the social network structure would change as reflected by an increased number of DBDM relationships.

We studied which changes occurred during the course of the intervention. As a result of the fact that it proved to be impossible to have a control group as a counterfactual we cannot claim causality between the observed changes and the intervention. Our first hypothesis reads as follows: during DBDM implementation the number of relationships regarding all DBDM topics (reflected by 'density') will increase.

DBDM will not only be discussed more, but also in a more intense manner, meaning that teachers have a closer look at their students' achievement, their achievement goals, and instructional approaches, and will discuss these more in detail with their colleagues. Therefore, it was expected that the number of mutual relationships (reciprocity) regarding DBDM would increase during the intervention. Hypothesis 2: during the DBDM implementation the number of reciprocal relationships regarding DBDM topics will increase.

Because the intervention was aimed at the entire school team and not just at one person within the team, it was expected that more team members would become knowledgeable about DBDM. We therefore expected that the academic coach would not be the only central person in the network, and that more equally divided DBDM relationships would be found. This was expected to lead to lower centralization measures. Hypothesis 3: during the DBDM implementation the network regarding DBDM topics will be less centralized around one, or a few influential persons compared to the start of the intervention.

School characteristics, such as the number of low-SES students, or school size might influence how collaboration patterns are formed. In large school teams, the total number of possible relationships is much larger than in small schools. As a result the pace of the information flow between the large number of teachers is slower than in smaller school teams. Hypothesis 4: in large schools density is expected to be lower.

In schools with more than average low-SES students, student achievement is usually lower (Mullis, Martin, Foy, \& Arora, 2012), and teachers in those schools feel a stronger need to work together to improve student achievement. Therefore, it was expected that schools with more than average low-SES students, would have more developed networks at the start of the DBDM implementation compared to schools with more than average high-SES students. Hypothesis 5: schools with more low-SES students than average, will have more developed networks compared to schools with on average more high-SES students.

The average age of the team members might also influence the density of the social networks. Moolenaar (Moolenaar, 2010) found that older and more experienced teachers are 
engaged to a lesser extent in work-related discussions compared to their younger colleagues. Spillane, Kim and Frank (2012) also found that more experienced teachers were less likely to receive advice or information in the school network. It was therefore expected that in - on average - younger teams networks would be more dense. Hypothesis 6: in 'young' school teams, collaboration regarding DBDM topics will be more dense.

\section{Methodology}

Data for this study were gathered from forty-one elementary (K-6) schools in the Netherlands that participated in the project. Data was collected at the start and after one year of DBDM implementation. In this section, first the sample and data collection are described, after which the section ends with a description of how the data were analyzed.

\section{Sample}

Four selection criteria were used to select the schools for the current study. First, schools had to participate in the intervention with the entire team, one school did not meet this criterion (teachers in the infant grades did not participate in the project). Second, the schools had to participate the whole school year. For organizational reasons, one school started in January 2013 instead of July 2012 and therefore also was excluded. Furthermore, school teams had to consist of at least six team members, because small teams could influence the network measures too much. Two school teams were excluded based on this criterion. Fourth and finally, because of their central position, school leaders had to fill out the questionnaire at both measurement occasions. The five schools in which this was not the case were excluded as well. After applying the selection criteria, 32 out of 41 schools remained in the sample.

The 32 sample schools were mainly located in urban areas throughout the Netherlands, and served a student population ranging from 69 to 545 students in the ages of 4 to 13. Compared to all Dutch schools, participating schools had relatively many students from a lower-SES background, and the average number of students in the participating schools was slightly larger than the national average, which is presumable caused by the exclusion of schools with small teams.

Data was collected among all team members, during the first and last meeting of the first intervention year. School teams ranged in size between 8 and 29 staff members. Of 616 elementary school staff members at the start of the project, 594 completed the survey (response rate 96.5\%; range 86\%-100\%). Of 596 staff members present at the last meeting 546 completed the survey (response rate 92\%; range 74\%-100\%). A matched dataset was constructed that included all staff members from whom data had been obtained at both measurements, resulting in a dataset of 512 respondents and an overall response rate of $89.2 \%$.

Of the matched sample about $87 \%$ of respondents was female, and the age of staff members ranged from 22 to $64(M=43.9, S D=12.0)$. Because two of 32 schools were located at two locations, 34 respondents fulfilled the role of school leader, 38 respondents were internal academic coaches, and 412 were teachers. Twenty-three staff members fulfilled other functions; most of them were teaching assistants.

\section{Data Collection}

Social networks. To examine the changes in collaboration patterns within the school teams, a quantitative survey method was applied. Participants completed a questionnaire at 
the start of the intervention, and after one year. This method made it possible to generate and monitor collaboration trends during the course of the DBDM intervention. Building on earlier work (Moolenaar, Sleegers, Karsten, \& Daly, 2012), three types of network questions which reflect aspects of DBDM were selected for their appropriateness in measuring teacher interactions. For instance, as research on DBDM underlines the importance of discussing student achievement (Visscher \& Ehren, 2011) as a first network measure the prompt 'With which colleagues do you discuss student achievement and progress at least once a month?' was included. In addition, another important aspect of DBDM involves collaboratively setting achievement goals that teachers would like to accomplish. Therefore, the following network prompt was included: 'With which colleagues do you discuss the achievement goals you would like to accomplish at least once a month?'. Finally, in order to reach the established goals, teachers need to choose effective instruction strategies. Sharing and discussing effective instruction strategies with your colleagues is therefore a third important aspect of DBDM. The third network question therefore was: 'With which colleagues do you discuss the instructional strategies in your group/in the school as a whole at least once a month?'

A roster technique was used to elicit respondents' interactions with their colleagues. More specifically, a list of numbers was included that corresponded with a list of all colleagues. For each of these questions respondents were asked to tick the numbers corresponding with the colleagues with whom they interacted; the number of colleagues a respondent could tick was unlimited.

School characteristics. Next to the network measures, data was collected on team size, the average school-SES, and the average age of the team members. School-SES (categorized as high, medium and low) was based on the percentage of students that had been assigned extra 'weight' based on parental educational levels indicating low SES'.

\section{Data Analysis Strategy}

Social network measures. For each school the density, reciprocity and centralization measures were computed, before and after one year of DBDM implementation, using the UCINET 6.0 software package (Borgatti, Everett, \& Freeman, 2002).

The concentration of relationships in a social network (density) can range from 0 (no relationships at all) to 1 (all relationships are present). Reciprocity (the extent to which relationships in a network are mutual) also can range between 0 (no reciprocal relationships) and 1 (all relationships are reciprocal). Centralized networks finally are centralized around one or more 'popular' actors. Centralization of networks ranges from 0 (the relationships are dispersed equally among all network actors) to 1 (the network is completely centralized).

This resulted in nine dependent variables: density, reciprocity, and centralization of all three network types.

Testing the hypotheses. Since the three network types (achievements, goals and strategies) together were considered to form the basis of DBDM, and were measured twice within a school team, multivariate linear regression was conducted with six dependent variables. For each of the network measures (density, reciprocity, and centralization) a multivariate linear analysis was conducted, using the Car package in R (Fox, Friendly, \& Weisberg, 2013). The explanatory variables team size, school-SES and average team age were

1 Students are assigned extra 'weight' if their parents are from a lower educational background. Students can get an extra weight of 0.3 (maximum parental educational level: lower vocational education), or 1.2 (maximum parental educational level: primary education, or special needs education). Schools receive additional funding based on student weights as it is assumed that schools with students with student weight have a more difficult job to do. 

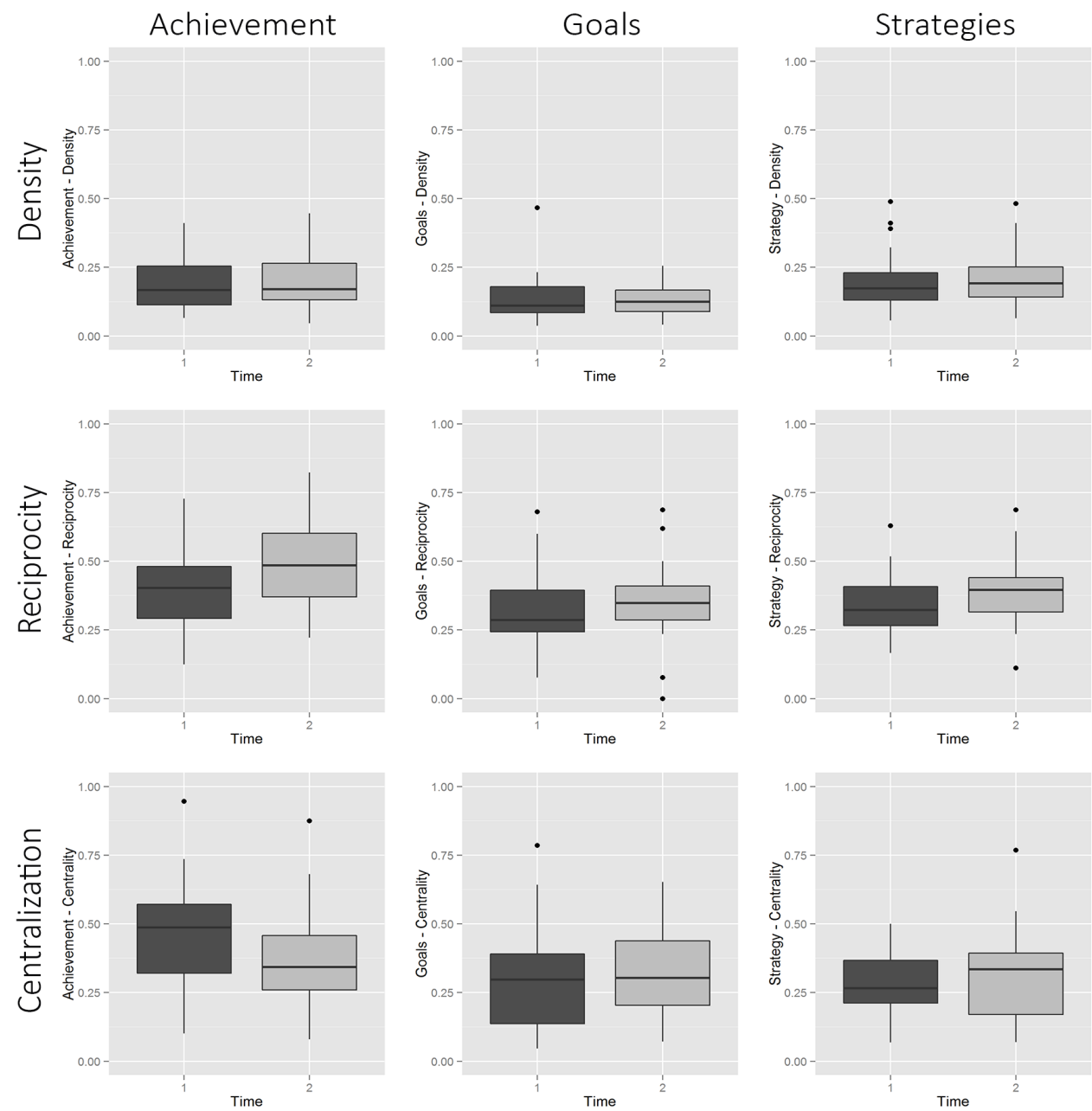

Figure 2. Boxplots of the three DBDM network types per network measure (density, reciprocity, centralization) before and after one year of DBDM implementation ( $N=32)$

Table 3. Means (and standard deviations) of the three DBDM network measures at T1 and T2 $(N=32)$

\begin{tabular}{lcccccc}
\hline & \multicolumn{2}{c}{ Achievement } & \multicolumn{2}{c}{ Goals } & \multicolumn{2}{c}{ Strategies } \\
& $\mathbf{T 1}$ & $\mathbf{T 2}$ & $\mathbf{T 1}$ & \multicolumn{1}{c}{ T2 } & T1 & T2 \\
\hline Density & $18.7(8.9)$ & $19.6(9.2)$ & $13.7(8.1)$ & $13.0(5.7)$ & $19.6(9.9)$ & $21.1(10.5)$ \\
Reciprocity & $39.9(13.8)$ & $48.4(13.8)$ & $31.5(13.9)$ & $35.6(13.3)$ & $34.5(10.4)$ & $38.6(11.5)$ \\
Centralization & $45.5(19.1)$ & $37.3(18.3)$ & $30.0(18.7)$ & $34.8(19.7)$ & $28.5(11.9)$ & $31.5(15.5)$ \\
\hline
\end{tabular}


used to explain differences in network measures between schools. The factor variables 'time' and 'network type' were used to explain differences between measurements within a school over time. The network measures were observed at the beginning and at the end of the first intervention year, which corresponds to a repeated-measure design with two measurements for each network type.

After conducting the multivariate linear analysis and defining appropriate contrast matrices, multiple hypotheses were tested to identify differences between network measures across schools, across network types, and over time. The hypotheses were tested using the multivariate test statistic, Wilk's Lambda, which is known to be F-distributed under the null hypothesis. The shortened $\mathrm{R}$ script of the analyses is presented in Appendix $\mathrm{F}$.

\section{Results}

\section{What Collaboration Looked Like Before the DBDM Implementation}

Before answering the hypotheses, we studied what collaboration looked like before the DBDM implementation. Figure 2 depicts boxplots of network measures (density, reciprocity, and centralization) per network type (achievement, goals, instructional strategies), before, and after one year of DBDM implementation.

Means and standard deviations per network measure, per network type are shown in Table 3. Prior to the DBDM intervention 'achievement', and 'instructional strategies' networks were on average slightly denser than the 'goals' networks. Almost $20 \%$ of all possible relationships were observed in the 'achievement' and 'instructional strategies' networks, compared to $14 \%$ of all possible 'goals' relationships. The significant main-effect of type in the multivariate analysis (Table 4) confirmed this finding. The densities of the three network types differed significantly from each other. Contrasts showed that the number of relationships regarding both 'discussing student achievement' and 'instructional strategies' was statistically significantly higher than regarding 'discussing performance goals'.

On average, the proportion of mutual relationships (reciprocity) was highest at T1 for discussing student achievement $(M=39.9, S D=13.8)$, and lowest for discussing goals $(M=31.5$, $S D=13.9$ ). Finally, the results at T1 suggested that networks concerning student achievement tend to be more centralized than networks with respect to discussing 'instructional strategies' and 'performance goals'. In other words, the former network was more centralized around a few influential teachers, whilst the latter two networks were more dispersed among staff members.

\section{How Collaboration Develops During the DBDM Intervention}

The first three hypotheses were about the transformation of the networks during the DBDM intervention. It was expected that density and reciprocity increased, and that centrality decreased. Changes in density, reciprocity and centralization were tested (the results can be found in Table 4). The number of relationships (density) remained stable during the DBDM implementation. For at least one of the network types the number of reciprocal relationships changed during the intervention. By testing contrasts, we found that the number of reciprocal relationships for the 'achievement' network increased significantly ( $\beta=.09, F=.69, p=<.001)$, but not for the 'goals', and the 'instructional strategies' networks. Furthermore Table 4 shows a significant time*type interaction effect for centralization; contrasts showed that centralization decreased for the 'student achievement' network ( $\beta=-.08, F=.77, p=.01)$, but not for the 'goals' and the 'instructional strategies' network. 
Table 4. Multivariate tests on predictors of DBDM network measures (density, reciprocity, centralization)

\begin{tabular}{|c|c|c|c|c|c|c|}
\hline \multirow[b]{2}{*}{ Effect } & \multicolumn{2}{|r|}{ Density } & \multicolumn{2}{|r|}{ Reciprocity } & \multicolumn{2}{|r|}{ Centralization } \\
\hline & $\Lambda$ & $F(d f 1, d f 2)$ & $\Lambda$ & $F(d f 1, d f 2)$ & $\Lambda$ & $\mathbf{F}$ \\
\hline Intercept & .07 & $366.09(1,28)^{* *}$ & .05 & $591.5(1,28) * *$ & .08 & $309.7(1,28)^{* *}$ \\
\hline \multicolumn{7}{|l|}{ Between schools } \\
\hline Team size & .42 & $39.29(1,28) * *$ & .96 & $1.03(1,28)$ & .81 & $6.56(1,28) *$ \\
\hline School-SES & .98 & $.53(1,28)$ & 1.00 & $0.02(1,28)$ & .86 & $4.67(1,28) *$ \\
\hline Average age & .96 & $1.17(1,28)$ & .97 & $.71(1,28)$ & .76 & $8.91(1,28) *$ \\
\hline \multicolumn{7}{|l|}{ Time } \\
\hline$\overline{\text { Time }}$ & .98 & $.43(1,28)$ & .71 & $11.25(1,28) * *$ & 1.00 & $.00(1,28)$ \\
\hline Time*Team size & 1.00 & $.07(1,28)$ & 1.00 & $.01(1,28)$ & 97 & $.85(1,28)$ \\
\hline Time*School-SES & .99 & $.28(1,28)$ & .99 & $.33(1,28)$ & .98 & $.71(1,28)$ \\
\hline Time*Average age & .97 & $.92(1,28)$ & .98 & $.46(1,28)$ & 1.00 & $.00(1,28)$ \\
\hline \multicolumn{7}{|l|}{ Type } \\
\hline Type & .25 & $41.54(2,27) * *$ & .53 & $12.06(2,27) * *$ & .33 & $27.25(2,27) * *$ \\
\hline Type*Team size & .72 & $5.14(2,27) *$ & .95 & $.74(2,27)$ & .92 & $1.21(2,27)$ \\
\hline Type*School-SES & 91 & $1.32(2,27)$ & .87 & $2.09(2,27)$ & .82 & $2.97(2,27)$ \\
\hline Type*Average age & 1.00 & $.04(2,27)$ & .98 & $.27(2,27)$ & .91 & $1.26(2,27)$ \\
\hline Type*Time & .88 & $1.87(2,27)$ & .94 & $.93(2,27)$ & .66 & $6.88(2,27) * *$ \\
\hline
\end{tabular}

${ }^{*}<.05 ;{ }^{* *}<.01$

\section{How Schools' Background Variables Influence DBDM Collaboration}

In order to test hypotheses 4, 5 and 6 about the background variables influencing DBDM collaboration, the explanatory variables team size, school-SES and average age of the team were included in the model. The main effects in Table 4 of these explanatory variables on the three network measures explained differences between schools. For all network types the size of the team had a negative effect on density. Contrasts showed that the size of the effect was largest for 'discussing instructional strategies' ( $\beta=-.025, F=.49, p=<.001)$ and 'student achievement' ( $\beta=-.024, F=.40, p=<.001)$, and slightly smaller for 'discussing goals' ( $\beta=-.016$, $F=.51, p=<.001)$. This explained the significant interaction effect between network type and team size in Table 5. The findings suggested that the larger the team, the smaller the proportion of actual relationships. Furthermore, team size influenced centralization: in larger teams the number of relationships was more equally dispersed.

A small significant effect of school-SES was found for centralization. Contrasts showed that this effect only yielded for the 'goals' networks ( $\beta=.21, F=.77, p=.01)$. This means that the higher the proportion of low-SES students in the school, the more centralized the network. In other words, in schools with many low-SES students relationships regarding 'discussing goals' were more centralized around one or a few influential persons.

The average age of the team was found to have a significant effect on centralization: the higher the average age, the more equally dispersed the relationships in the network. This effect was the same for all network types.

Background variables team size, school-SES, and average age did not influence the development of the collaboration patterns. 


\section{Conclusions and Discussion}

Many scholars in the field consider a collaborative school culture as a precondition for the successful implementation of a (DBDM) reform project. However, few researchers have studied the impact of such a project on collaboration patterns within schools. In the present study, the transformation of DBDM collaboration patterns during a DBDM intervention were investigated. This was done by studying the density, reciprocity and centralization of three DBDM networks in 32 elementary schools in the Netherlands.

Table 5. Study hypotheses, results and findings

\begin{tabular}{lll}
\hline Hypotheses & Results & Findings \\
\hline Hypothesis 1: during the DBDM & & No significant effect for all \\
implementation the number of & & three network types (discussing \\
relationships regarding all DBDM topics & Not & $\begin{array}{l}\text { student achievement, goals and } \\
\text { (reflected by 'density') will increase. }\end{array}$ \\
& supported &
\end{tabular}

Hypothesis 2: during the DBDM implementation the number of reciprocal relationships regarding DBDM topics will increase.

Hypothesis 3: during the DBDM implementation the network regarding DBDM topics will be less centralized around one or a few influential persons compared to the start.

Hypothesis 4: in schools with large teams density is lower.

Hypothesis 5: schools with more low-

SES students than the average, will have more developed networks compared to schools with on average more high-SES students

Hypothesis 6: in young school teams collaboration regarding DBDM topics is denser.
The number of reciprocal relationships increases for the

Partially student achievement network, supported but not for the goals and for the instructional strategies network.

Centralization decreases for the student achievement network, Partially supported but not for the goals and instructional strategies network.

The number of team members influences the proportion of relationships regarding DBDM

Supported (density): the larger the team, the lower the density.

No significant effect

Not supported

Not

No significant effect

supported 
Results of hypotheses testing are summarized in Table 5. In order to test our hypotheses, first the collaboration patterns before the intervention were studied. Since schools chose to participate in a DBDM intervention themselves, it was expected that interaction regarding DBDM topics such as discussing 'student achievement', 'goals' and 'instructional strategies' was not everyday practice, and that this would be reflected by low density, low reciprocity and high centralization. Our findings show that in terms of discussing 'student achievement' and 'instructional strategies', approximately $20 \%$ of all potential relationships had actually been formed, while 'achievement goals' were discussed little. In all DBDM networks, not even a fifth of all potential relationships was formed. This is in line with our expectations, but also problematic, since such a sparse network could hinder the development of DBDM within the school.

Furthermore, the percentage of reciprocal relationships was less than $40 \%$ for all three DBDM networks, indicating that fewer than half of all possible relationships were reported by both actors. Since reciprocal relationships are associated with the transfer of complex knowledge (Kilduff \& Tsai, 2003) we consider this level of network reciprocity to be low. Since DBDM demands from team members that they work together to improve student achievement, this low level of reciprocity might reduce the effectiveness of DBDM.

Finally, it was expected that the initial DBDM networks would be very centralized, due to the expected central position of the academic coach in the network. Results show that this is especially true for the 'achievement' network, suggesting that those relationships in which student achievement is discussed are organized around one or a few influential persons (probably the academic coach(es)) within a school. However, the 'instructional strategies' network was less centralized. An explanation for this might be that teachers discuss these instructional strategies not (only) with a person who they consider to be an expert, but also with teachers in the same grade level, or in the classroom next door. However, current analyses address the school level, further analysis at the individual teacher level would be needed to confirm this.

Even though we cannot claim that the DBDM networks transform as a result of the DBDM intervention the observed network changes are interesting and can provide a basis for future studies that allow causal claims. It was expected that collaboration regarding DBDM topics like 'discussing student achievement', 'discussing performance goals', and 'discussing instructional strategies' would grow during the intervention. This was expected to be reflected by increased numbers of relationships (hypothesis 1), increased numbers of reciprocal relationships (hypothesis 2), and a decreased centrality of the network (hypothesis 3). The latter meaning that the relationships are dispersed more equally within the team.

Hypothesis 1 is not confirmed. Growth in the number of DBDM relationships is not observed. The number of mutual relationships staff members reported did increase for the 'student achievement' network, but not for the 'goals', and the 'instructional strategies' network (partial support hypothesis 2). This might imply that compared to the start of the intervention, more complex knowledge regarding student achievement is shared now between staff members. Remarkably, centralization in both the 'goals', and the 'strategies' networks remained stable. In turn, in the 'student achievement' networks, relationships became more equally divided (hypothesis 3 partially supported).

In sum: collaboration within school teams changes during the implementation of the DBDM reform to some extent. Although, the number of relationships within the network remains stable, DBDM collaboration changes within the school teams, reflected by increased reciprocity (more mutual relationships between school staff members), and decreased 
centrality (more dispersed relationships within school teams), especially for the 'achievement network'.

As far as the nature of the collaboration is concerned the study points to the fact that changes during the DBDM intervention were especially found with respect to discussing 'student performance'. Thus, collaboration with respect to 'performance goals' and 'instructional strategies' is not influenced much during the DBDM intervention. It seems that learning about the possibilities and use of student performance data went together with the strongest changes in teacher collaboration. Setting goals cooperatively, and discussing and adapting instructional approaches both seem to change less easily. The utilization of student performance data throughout the school is something quite new and revolutionary for Dutch teachers and schools; instructional decisions are considered to belong to the discretion of individual teachers.

Great variability is found between the school networks. It was expected that school characteristics, such as the proportion of low-SES students in schools, average team size, and the average age of the team members influenced the DBDM networks. However, multivariate analysis neither show an effect of SES (hypothesis 5, rejected) nor of the average team age (hypothesis 6, rejected) on the density of the networks. The average age does have an effect on centralization: the older the team, the more equally dispersed the relationships in the network. Possibly, staff members in teams with less experienced, younger teachers, tend to reach out more to a DBDM expert, compared to staff in teams with more experienced (older) teachers. Furthermore, school-SES does influence centralization, but only for the 'goals' networks: in schools with a high percentage of low-SES students, networks were more centralized around one or a few central persons. An explanation for this finding remains unsure.

Finally, the size of the team is related to collaboration density: the smaller the team, the denser the network (hypothesis 4, confirmed). In larger teams, there might be fewer opportunities to share knowledge and expertise with each other since large teams have more links, through which the information has to flow. Moreover, the question is whether it is possible and desirable to discuss DBDM topics in a team wide way in large teams. In small teams with only one or two teachers per grade, the need to share DBDM knowledge and skills with the entire team is stronger, compared to a school with more than four or five teachers per grade. In such a large school, subgroups of teachers within the same grades are often formed in order to discuss DBDM topics.

\section{Limitations and Areas for Future Research}

Although the findings of this study provide insight into the changes that occurred during a DBDM intervention, it is not correct to claim that these changes occurred due to the intervention since all respondents participated in the DBDM intervention, and a control group could not be included in this research. Further research is needed to clarify the changes in networks as a result of DBDM interventions using longitudinal experimental designs.

Furthermore, the study deals with the perceptions of school team members about their interactions with colleagues. These perceptions may change due to the intervention, which might influence their interpretation of the network questions. For example, the participants were familiar with the three DBDM subjects at the start of the training, but due to the training the interpretation of 'discussing student achievement' may have differed at T2 from how it was interpreted at T1. Teachers may have talked about their student outcomes at $\mathrm{T} 1$, but the content of those conversations may differ after one year of intervention. 
This might explain why network density did not change during the intervention. In future research, it will be important to not only study whether teachers talk with each other about DBDM topics, but also to investigate the frequency and the nature of such interactions.

Based on the literature (both from organizational science and from educational science) we assumed that the ideal DBDM network would be dense, decentralized and reciprocal. However, this study does not confirm this. It could for example be argued, that a network centralized around one or two data experts is preferably for sharing data use knowledge (Marsh et al., 2015). In order to obtain a better understanding of 'good' collaboration in the context of DBDM, in a follow-up study the relationships between network characteristics and student achievement will be examined. Hopefully this relationship will also be studied more in other research projects. Furthermore, to better understand network changes, for example why the 'strategies' network, and the 'goals' network become more centralized, it would be interesting to study the network measures at the individual staff member level. This might, for example, provide insight into the role of experts such as the academic coach, or the school leader within the team. To do so, longitudinal network analysis using actor-oriented statistical models for network evaluation (Snijders, Steglich, Schweinberger, \& Huisman, 2007; Snijders, 2001, 2005) have much potential. Such an advanced longitudinal analysis of social networks would also enable checking whether changes in relationships over time are different for different positions in a team. Several authors (Datnow, 2011; Levin \& Datnow, 2012; Moolenaar et al., 2010) suggest that the school leader can play an important role in DBDM. Centralization of school leaders within a team might change as a result of a DBDM intervention. Further analysis of the current dataset could give more insight into changes in networks related to the various positions within school teams.

To conclude, this first longitudinal study of its kind looking into the transformation of social networks during a school improvement intervention contributes to the knowledge base of social network theory within the context of schools. It indicates that networks within school teams do not only influence reform, but also that a reform can improve networks. Other similar reform initiatives should therefore consider including activities that promote collaboration among school staff during the reform. 
The transformation of schools' social networks 


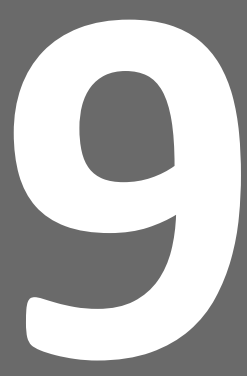

Why a Data-Based Decision Making Intervention Works in Some Schools and Not in Others 


\begin{abstract}
Background. Collaboration within school teams is considered to be important to build the capacity school teams need, to work in a data-based way. In a school characterized by a strong collaborative culture, teachers may have more access to the knowledge and skills for analyzing data, teachers have more opportunity to discuss the performance goals to be set, and they also can share effective teaching strategies to achieve those goals. Although many studies on data-based decision making (DBDM) foreground the importance of teacher collaboration, our knowledge on what such collaboration looks like and how such collaboration may change during a DBDM reform, remains limited.
\end{abstract}

Purpose. The current study uses a social network perspective to explore how collaboration in 32 elementary schools in the Netherlands takes shape in the interactions among teachers as they engage in a DBDM reform project.

Method. Schools' social networks were examined at the start of the intervention and after having participated one year in the DBDM reform. Social networks regarding three DBDM topics are examined: (1) discussing student achievement; (2) discussing the achievement goals; (3) and discussing instructional strategies. The density, reciprocity and centralization of these networks were calculated, and multivariate multiple regression analysis was used to analyze changes over time.

Findings. Findings suggest that teachers' DBDM related networks transform during the intervention, especially regarding the discussion of student achievement data: although the number of relationships remains stable, more reciprocal relationships are formed, and this network becomes less centralized around one, or a few influential staff members. 
School A and school B are two primary (K-6) schools, belonging to the same school board in a suburban area in the Netherlands. Both schools participated in the same two-year intervention to implement and sustain DBDM in their schools, aimed at improving student performance. After two years, the staff of school A is really enthusiastic and sees the added value of working in a data-based way as student performance has significantly improved compared to the period before the intervention. However, in school B the results are not that promising; it looks like the intervention did not affect student performance at all. Teachers in school B perceive DBDM as time consuming and involving a lot of paperwork, whereas it failed to support their educational practice.

Why was the same DBDM intervention effective in school A, but not in school B? In the evaluation of this two-year DBDM intervention, we found evidence of a positive average intervention effect on student outcomes (chapter 2 and chapter 3). However, we also found considerable variation between intervention effects across schools. In this chapter, we study what educator and school organizational factors are associated with these differences in intervention effects. To do so, the ten schools with the strongest intervention effects were compared with the ten schools with the smallest intervention effects.

\section{Theoretical Framework}

In the following section, the conceptual model of this study is described step-by-step (as visually depicted in Figure 1). We start at the bottom of Figure 1 and gradually move upwards. First, we will discuss how DBDM is supposed to influence student outcomes. Second, the educator characteristics and school organizational features that supposedly influence DBDM are presented. Finally, we elaborate on how a DBDM intervention can improve DBDM.

\section{DBDM and Improved Student Outcomes}

DBDM is defined as: "teachers, principals, and administrators systematically collecting and analyzing data to guide a range of decisions to help improve the success of students and schools" (Ikemoto \& Marsh, 2007, p. 108). In Figure 1, DBDM is decomposed into four components: analyzing and evaluating data, setting SMART and challenging goals, choosing strategies to accomplish the goals set, and, finally, executing the chosen strategies. The ultimate goal of DBDM is to maximize the performance and outcomes of all students. In a number of studies DBDM influenced student outcomes positively (Campbell \& Levin, 2008; Carlson, Borman, \& Robinson, 2011; Konstantopoulos, Miller, \& van der Ploeg, 2013; K. M. Lai, Mcnaughton, Amituanai-toloa, Turner, \& Hsiao, 2009; May \& Robinson, 2007). However, researchers also argue that there is still much work to do in terms of investigating precisely how data use can reach its full potential (Kaufman, Graham, Picciano, Popham, \& Wiley, 2014). In other words, more insight into the conditions under which DBDM works is needed.

\section{Factors Influencing Successful (Implementation of) DBDM}

In the international DBDM literature, a broad range of features that might explain differences in DBDM effects are mentioned (Ikemoto \& Marsh, 2007; Mandinach, 2012; Schildkamp, Karbautzki, \& Vanhoof, 2014; Schildkamp \& Lai, 2013; Schildkamp \& Poortman, 2015; van Geel, Teunis, \& Visscher, 2016; Visscher \& Ehren, 2011). For the purpose of this study, we distinguish between educator and school organizational features that we consider most relevant for enhancing the implementation of all four DBDM components. 


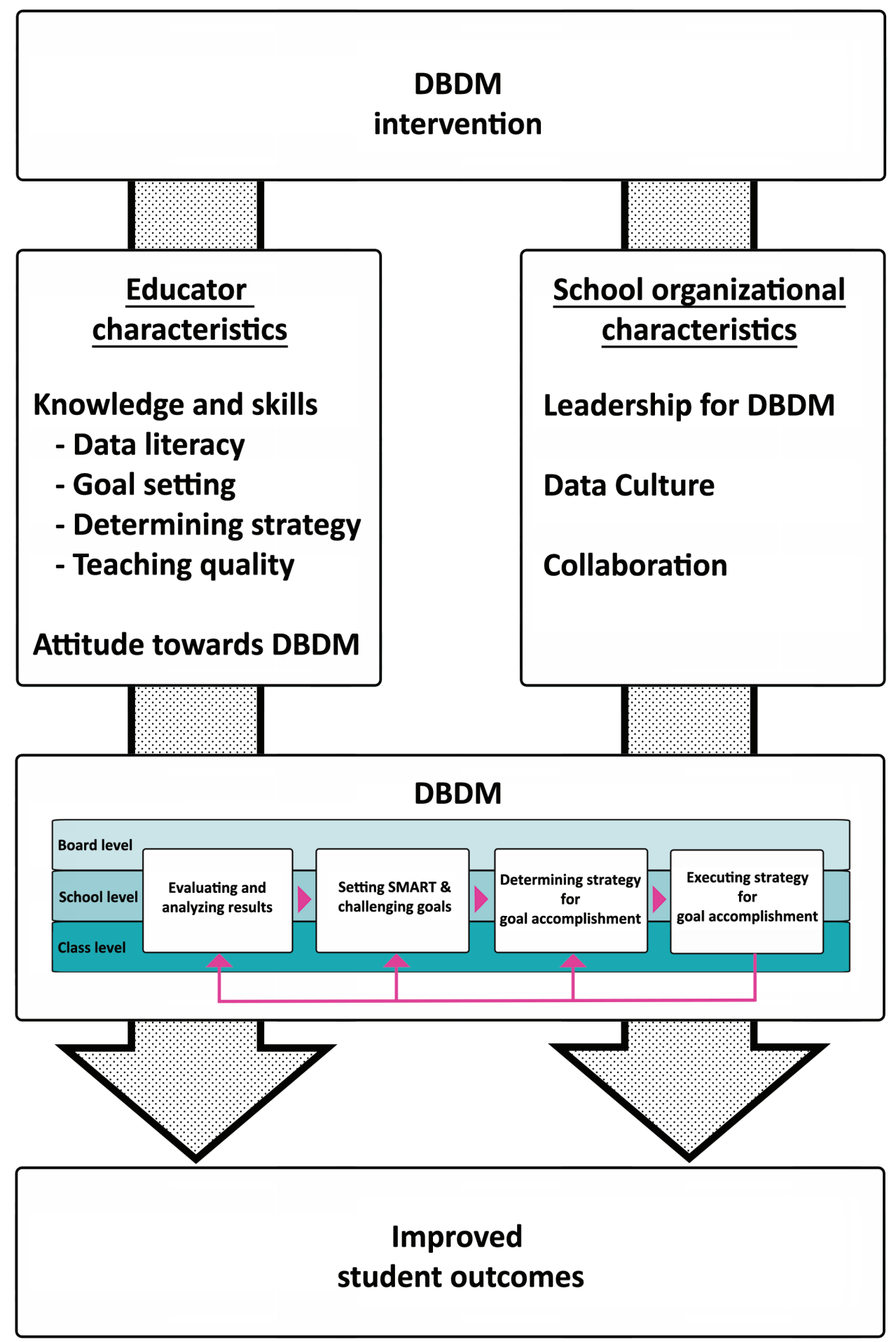

Figure 1. Conceptual model 


\section{Educator features.}

Educators' knowledge and skills. Educators' knowledge and skills regarding DBDM are important for successful DBDM. First, knowledge and skills regarding analyzing and interpreting data, also called 'data literacy', are essential. Educators need to know how to transform raw data into actionable knowledge, therefore skills such as collecting and organizing data, analyzing and summarizing data, and synthesizing and prioritizing data are needed (Bennett, 2011; Mandinach, Honey, \& Light, 2006).

Next, based on these data-analyses, users should know how to set SMART and challenging goals (Locke \& Latham, 2002), and they need to be able to choose effective strategies to meet these goals. Finally, users (more specifically, teachers) need to execute the strategies chosen in daily classroom practice (Anderson, Leithwood, \& Strauss, 2010; Visscher \& Ehren, 2011). In this matter, teaching quality comes into play. Next to the basic, more general dimensions of teaching quality, such as creating a safe classroom climate, efficient classroom management, and providing clear instruction (Fauth, Decristan, Rieser, Klieme, \& Büttner, 2014; Pianta, Hamre, Haynes, Mintz, \& La Paro, 2007), two other teaching skills that are important for DBDM should be mentioned here: challenging students and teaching them in a goal-oriented way (see also: chapter 5).

Educators' attitudes towards DBDM. A positive attitude towards DBDM of all team members is regarded as important for successful DBDM (Schildkamp \& Ehren, 2013; Schildkamp et al., 2014; Vanhoof, Van Petegem, \& De Maeyer, 2009). A negative DBDM attitude of (part of) the school team can severely hinder DBDM, as was found by Reeves and Burt (2006). A negative attitude of teachers towards testing was one of the major challenges principals faced when implementing DBDM in their schools, since teachers who did not see the value of testing did not use test results effectively (Reeves \& Burt, 2006).

\section{School organizational features.}

Leadership for DBDM. The school leader is considered to have a key role in the successful implementation of DBDM in multiple ways (Ikemoto \& Marsh, 2007; Levin \& Datnow, 2012a; Schildkamp \& Lai, 2013). First, the school leader can play an important role for the practical preconditions for DBDM, such as the selection of a proper student monitoring system, and providing teachers with time for DBDM activities and collaboration (Cosner, 2012; Datnow, 2011; Ikemoto \& Marsh, 2007; Schildkamp \& Lai, 2013). Second, by promoting and facilitating data use, a school leader can influence teachers' attitudes towards DBDM (Wayman, Cho, Jimerson, \& Spikes, 2012). Finally, by promoting a strong DBDM vision and by having clear rules and norms for data use, the data culture of the school can be positively influenced by the school leader (Coburn \& Turner, 2011; Marsh, 2012).

Data culture. A culture that fosters data use and is achievement oriented is regarded as essential for the implementation and sustaining DBDM (Ingram, Louis, \& Schroeder, 2004; Mandinach, 2012; Wayman, Midgley, \& Stringfield, 2006). According to Lai and Schildkamp (2013), DBDM is best implemented in schools in which team members feel the need to critically look at data, reflect on their own functioning, and, are open to changing their practice when data reveals a need to do so. However, it is not always easy to reflect on one's own functioning when feeling threatened (Marsh, 2012). A climate of trust is therefore essential in establishing a data culture within schools (Ikemoto \& Marsh, 2007; Marsh, 2012; Visscher \& Ehren, 2011). In such a culture, data are not used to judge but to support improvement. 
Moreover, an explicit data use vision which is clearly communicated by the school leader is an essential part of a data culture. Such a vision is characterized by "a group's appreciation for the importance and power that data can bring to the decision-making process" (Hamilton et al., 2009, p. 46).

Collaboration. Strong collaboration patterns can also support DBDM within a school (Ingram et al., 2004; Schildkamp \& Lai, 2013; Schildkamp \& Poortman, 2015). In highly collaborating teams, teachers have more access to knowledge and skills for analyzing data, and also have more opportunities to discuss performance goals, and share effective strategies for goal accomplishment. Strong collaboration thus enhances a teams' capacity to work in a data-based way (Farley-Ripple \& Buttram, 2015; Jimerson \& Wayman, 2015).

Limited empirical evidence. In sum, the DBDM literature presents a variety of factors that possibly influence the successful implementation of DBDM. However, there is still limited empirical evidence that these features really matter for improving student performance via DBDM.

\section{Improving DBDM with an Intervention}

Despite the increasing endorsement for data use from policy-makers and researchers, working in a data-based way is not common practice in education: educators struggle with selecting, analyzing and interpreting data, and they experience difficulties in connecting data with instructional decisions (Farley-Ripple \& Buttram, 2015), and find it hard to change their classroom practice according to those decisions (Means, Padilla, \& Gallagher, 2010; Schildkamp \& Kuiper, 2010). DBDM interventions are therefore needed to support schools in implementing DBDM. Such an intervention should be in line with the features of effective professional development (Desimone, 2009; Timperley, 2008; Van Veen, Zwart, \& Meirink, 2011).

Features of effective professional development. Since it takes time to learn and change, the duration of an intervention is important with respect to both the number of contact hours, and the time span over which the PD activity is spread (Desimone, Porter, Garet, Yoon, \& Birman, 2002). According to Timperley (2008), it takes typically one to two years for teachers to fully understand new beliefs and practices, and to change practice accordingly.

A clear link between theory and practice is also important, teachers should have the opportunity to gradually practice and implement what they have learned (Timperley, 2008; Van Veen et al., 2011).

Furthermore, collective participation (e.g. as a school team) in a professional development trajectory is positively correlated with the active participation of school staff in professional development activities (Garet, Porter, Desimone, Birman, \& Yoon, 2001; Lumpe, 2007). Van Veen, Zwart, and Meirink (2011) as well as Timperley (2008) argue that interaction with and collaboration between colleagues is important when implementing an innovation and mastering its content.

Finally, the implementation of a professional development intervention can benefit from externally developed reform designs, because they provide detailed guidelines for implementation (Desimone, 2002). The same applies to external experts, who involve teachers in discussing and developing their understanding of an innovation, and support 
teachers as they develop their understanding (Timperley, 2008). Staff also seem to support a reform more if they are actively engaged in co-constructing an innovation in their schools so that the changes fit their local context (Datnow, Hubbard, \& Mehan, 1998). Datnow and Castellano (2000) describe the adaptations teachers made to the Success for All program, when they felt their students' needs were not met by the prescribed program. Although such adaptations could affect program fidelity, some flexibility was necessary to ensure the continuing involvement of teachers.

\section{The DBDM Intervention}

A two-year team training, called 'the Focus intervention', was developed with the aim to implement and sustain DBDM within the whole school organization. In August 2011, the first cohort of 53 primary schools started the intervention (Focus II), after which in August 2012 a second cohort of 43 primary schools started the intervention (Focus III). In both cohorts, the same content was taught to the participants and the training included the same sequence of meetings.

Cohorts received trainings from different DBDM trainers, however, all trainers had been appointed by the University of Twente for this project. Furthermore, the implementation of the training was supervised by the first two authors of this article, who themselves were not directly involved in working with the schools.

Overview of the meetings. The design of the training program was based on the features of effective teacher professional development described in the previous sections. For instance, the intervention lasted for two school years, to allow schools to gradually change practices towards the full implementation of DBDM.

In the first year, the basic knowledge and skills for DBDM were covered in seven team meetings. Using their student monitoring system (meeting 1.1), team members analyzed their own student data, learned how to set goals based on these analyses (meeting 1.2), and make plans to achieve these goals (meeting 1.3, and 1.4). Next, the developed plans were executed by the teachers in their classrooms, and were thereafter evaluated in meeting 1.5. During meeting 1.5, the school leader presented the first results in terms of the achievements of the students on a standardized test. Meeting 1.6 focused on teacher peer consultation, teachers were provided with the knowledge and tools required for observing their colleagues in the classroom, and for providing them with feedback. Finally, in meeting 1.7, similarly to meeting 1.5 , student outcomes were discussed at the school level and plans were made for the second school year. In this second year, all steps of the DBDM cycle (as depicted in Figure 1) were executed twice. This year included five team meetings in which schools chose to broaden their scope to a new subject, or to deepen DBDM for the subject they had already worked on throughout the first year. Meeting 2.1 was aimed at analyzing student performance data and diagnosing students' learning for the new subject, or, (for those schools who chose to continue to work on DBDM for the same subject during the second intervention year) at focusing on issues the school faced in implementing DBDM during the first year. Meeting 2.2 was a coaching session, in which the external project trainers, together with the school internal academic coach, observed how teachers executed DBDM within their classroom, the trainers then provided the teachers with individual feedback. This was followed by a team meeting in which trainers and teachers shared their classroom observation experiences. In meeting 2.3 (comparable with meetings 1.5 and 1.7), student outcomes were discussed with all school team members. Halfway through the second year, schools again had the 
opportunity to broaden their scope to a new subject. Meeting 2.4 in Focus II focused on this new subject and on issues the school had faced in implementing DBDM during the first year. In Focus III, a second lesson observation round was included. Finally, in meeting 2.5 student results were discussed for the last time as part of the intervention and schoolwide plans were developed to sustain DBDM within the school.

In addition to the team meetings, the project trainer discussed schools' progress with the school leaders and (if applicable) the academic coach twice a year. In these meetings, the importance of fulfilling the practical preconditions for DBDM was stressed and schools' progress regarding DBDM was discussed. Furthermore, school leaders were supported in preparing the team meetings during which the results of students in the various grades was evaluated, as well as educational strategies to improve student learning and achievement. During the meetings, the school leader and the academic coach were also provided with an instrument (and accompanying training activities) for observing teacher classroom teaching to enhance the structural monitoring of teaching quality.

To ascertain that the DBDM training was as consistent as possible across schools and trainers, each meeting had a central topic which was the same for every participating school (e.g., analyzing test score data from the student monitoring system, goal setting and developing instructional plans, discussing student progress, the goals for the next period). The content of these meetings was fixed for all schools, the same PowerPoint slides were used and the same exercises were conducted. Prior to each meeting, the trainers discussed the content for that specific meeting intensively to assure that each of them would present the information in the same way. The time a trainer spent on a specific topic within a meeting varied somewhat between schools, as school teams' prior knowledge, team members' needs, and the subject chosen by a school varied.

Differences between Focus II and Focus III. Focus II and Focus III differed slightly. First, in Focus II all schools started the intervention focusing on 'mathematics'. In the second year, Focus II schools had the possibility to broaden their scope to 'spelling'. In contrast, Focus III schools could choose the subject they wanted to start with in the first year: mathematics, spelling, reading comprehension or vocabulary. In the second year, schools could broaden their scope to a second subject. Second, in Focus III an extra observation round was included in the second year, instead of a meeting (as in Focus II). Finally, in the first year of the Focus II intervention, meetings 1.2 and 1.5 which focused on mathematics content, were (partly) taught by external experts. Based on feedback from the Focus II schools, in Focus III these meetings were delivered by the project trainers.

\section{Research Question}

The fact that the DBDM intervention worked in school B but not in school A could have been caused by a number of factors. We studied those factors in two ways, the school leaders of the participating schools were interviewed and reports from the project trainers were examined. This allowed us to answer the following research question: Which educator and school organizational factors, as perceived by trainers and school leaders, have promoted and/or hindered DBDM implementation in their schools?

Furthermore, we were curious whether the promoting and hindering factors mentioned by school leaders and trainers would be similar to the factors mentioned in DBDM literature (Reeves \& Burt, 2006; Schildkamp \& Poortman, 2015; van Geel et al., 2016).

Second, based on the theoretical framework for this study (reflecting the, in our 
opinion, most important factors for explaining DBDM intervention success) the educator characteristics and school organizational characteristics of schools with strong intervention effects were compared with those of the schools without intervention effects.

Here, the underlying research question was: What differentiates schools that benefit from the DBDM intervention from those that do not benefit from the intervention?

To our knowledge, this is the first study to relate promoting and hindering DBDM factors to student achievement growth.

It was expected that staff in schools with strong intervention effects ('strongeffect schools') had more DBDM knowledge and DBDM skills at the end of the intervention compared to the group of schools with no intervention effects (reflected by higher data literacy, and higher teaching quality). Furthermore, their attitude towards DBDM during the intervention was expected to be more positive than the schools without intervention effects. Regarding school organizational characteristics, it was expected that in strong-effect schools, school leaders would be perceived as more achievement oriented, that the data-culture in such schools would be stronger, and that teachers in strong-effect schools would collaborate more on DBDM topics such as the discussion of student achievement, achievement goals, and appropriate instructional strategies.

\section{Methodology}

For the current study, data from 20 out of 101 primary schools in the Netherlands which had participated in the Focus intervention were used. To answer the research questions, a mixed method approach was applied to study the factors that contributed to the variation in intervention effects between schools. In this section, the sample is described first, followed by an elaboration of the data collection procedure, and the section ends with a description of how multiple instruments were used to answer the research questions.

\section{Sample}

A total of 101 primary schools participated in a two-year DBDM intervention. The first cohort of 53 schools started in the school year 2011-2012, the second cohort of 48 schools started in the school year 2012-2013. Based on their calculated intervention effect on student mathematics achievement, expressed in terms of improved student achievement growth as described in chapter 2 and chapter 3, schools in each cohort were ranked from the lowest (or no) intervention effect to the highest intervention effects. From the 2011-2012 cohort, one outlier was excluded as this was a very small school with unusual high levels of student achievement at the start of the intervention.

Next, from each of the two cohorts, the upper and lower five schools were selected. This resulted in 20 schools, of which the characteristics are presented in Table 1 . For the purpose of this article, the strong-effect schools are coded SE1 to SE10 and the no-effect schools are coded NE11 to NE20. This sample of 20 schools was used to answer both research question 1 and 2 .

As can be found in Table 1, the no-effect schools and the strong-effect schools differ from each other on a number of school characteristics. In chapter 2 and chapter 3 , we already noted a significant interaction effect of school-SES, also presented in Table 1, showing that the majority of the strong-effect schools have a low-SES student population, whereas a majority of no-effect schools have a medium-SES population. Additionally, Table 1 shows that strong-effect and no-effect schools differ from each other in school size, 
Table 1. School context characteristics of strong-effect schools $(N=10)$ and no-effect schools $(N=10)$

\begin{tabular}{|c|c|c|c|c|c|}
\hline & & $\mathbf{N}$ & $(\%)$ & $\mathbf{N}$ & $(\%)$ \\
\hline \multirow[t]{3}{*}{ School size } & Small (<150) & 2 & $(20 \%)$ & 5 & (50\%) \\
\hline & Medium (150-350) & 8 & $(80 \%)$ & 3 & (30\%) \\
\hline & Large (>350) & 0 & $(0 \%)$ & 2 & $(20 \%)$ \\
\hline \multirow[t]{3}{*}{ School-SES } & High & 1 & $(10 \%)$ & 3 & $(30 \%)$ \\
\hline & Medium & 3 & $(30 \%)$ & 6 & $(60 \%)$ \\
\hline & Low & 6 & $(60 \%)$ & 1 & $(10 \%)$ \\
\hline \multirow[t]{3}{*}{ Urbanization } & Rural & 1 & $(10 \%)$ & 4 & $(40 \%)$ \\
\hline & Suburban & 5 & $(50 \%)$ & 5 & $(50 \%)$ \\
\hline & Urban & 4 & $(40 \%)$ & 1 & $(10 \%)$ \\
\hline \multirow{5}{*}{$\begin{array}{l}\text { Intervention } \\
\text { trajectory }\end{array}$} & M-M-M & 6 & $(60 \%)$ & 1 & $(10 \%)$ \\
\hline & $M-M-X$ & 2 & $(20 \%)$ & 1 & $(10 \%)$ \\
\hline & $M-X-X$ & 1 & $(10 \%)$ & 5 & $(50 \%)$ \\
\hline & X-M-M & 1 & $(10 \%)$ & 2 & (20\%) \\
\hline & X-M-X & 0 & (0\%) & 1 & $(10 \%)$ \\
\hline
\end{tabular}

Note. The intervention trajectory was chosen by the schools. Schools decided whether they wanted to focus on Math (M) during the first intervention year or another subject such as spelling, vocabulary, or reading comprehension $(X)$. In the second year, schools were offered the opportunity to add another subject or stick to the same subject. Halfway through the second year, schools could again choose to add another subject or stick to the same subject. This resulted in five different intervention trajectories.

Table 2. Overview of data collection

\section{Educator characteristics \\ School organizational characteristics}

Report of the trainer

(T3)

Interview with school leader

(T3)

Data literacy test

(T1/T3)

Student survey about teaching quality

(T1/T2b)

Teacher questionnaire about attitudes towards DBDM

(T1/T2a/T3)

Teacher questionnaire about school leadership

(T1/T2a/T3)

Teacher questionnaire about school culture

(T1/T2a/T3)

Social network survey

(T1/T2a/T3) 
urbanization and intervention trajectory. The latter refers to the subjects the schools started with, and the subjects they added to their trajectory throughout the intervention period. Apparently, schools which chose to focus on mathematics for both years, reached the highest intervention effects for mathematics while the schools which chose to add a new subject in the second intervention year had no student achievement effects at all. Although, the impact of the context factors (school-SES and intervention trajectory) is important, the current study was especially meant to clarify which educator and school organizational features explain differences in the impact of the intervention between schools.

\section{Instruments and Data Collection}

Multiple instruments were used to gather data about the factors potentially influencing DBDM implementation (see Table 2). Data collection took place at the start of the intervention (T1), the end of the first intervention year (T2a), the start of the second intervention year (T2b), and at the end of the second intervention year (T3).

Qualitative data. To answer research question 1 and gain insight into school leaders' experiences regarding the factors contributing to and hindering the implementation of the DBDM intervention, interviews with school leaders were held using the storyline-method (Beijaard, Driel van, \& Verloop, 1999; Huizinga, Handelzalts, Nieveen, \& Voogt, 2015). This method offered school leaders the opportunity to reflect on their DBDM implementation process experiences during the entire intervention period. First, the trainer explained the storyline method to the school leader and in some cases: the academic coach or other members of the school management team. They were provided with an empty graph. Time (in months) was displayed on the x-axis and the $y$-axis ranged from 1 to 10 . School leaders were asked: "how would you rate the process of implementing DBDM in your school during the Focus-project?" They could rate the process with a score between 1 and 10. School leaders answered this question by plotting a storyline in the empty graph, starting at 'now' (thus, at the end of the project), and then, while thinking back to the start of the project, rate the various time points of the process by giving grades between 1 and 10 .

After they had plotted the storyline, school leaders were asked to which factors had caused the peaks and valleys in the storyline they had drawn (e.g. the acquisition of data analysis competences caused enthusiasm versus an intervention meeting of which the content was considered to be too repetitive by participants, demotivating staff). Additionally, the interviewer asked which of the more general factors had promoted or hindered DBDM implementation (e.g. general collaboration within a school team versus poor teaching quality). The interviewer made notes during the interview and summarized the outcomes for each school. These notes and summaries were used for our study.

In addition, prior to their interviews with a school leader, trainers wrote down their own experiences per school in a report using the same storyline method. In this report they, answered the same questions as the school leaders. These trainer reports were also used for this study.

Quantitative survey data. Participants filled out a paper and pencil questionnaire at T1, T2a, and T3. In this questionnaire, 13 items measured school leadership (see Appendix D) and ten items were used to measure a schools' data culture (see Appendix E). Both questionnaire parts were scored on a four-point Likert-scale, ranging from 'totally disagree' to 'totally agree'. To examine collaboration patterns within the school teams, three social 
network questions had been included in which respondents were asked to name the colleagues with which they, at least once a month, discussed 1) student achievement and progress, 2) their achievement goals and 3) instructional strategies. Finally, the questionnaire included 29 items measuring teachers' attitudes towards DBDM. The items were divided over four scales: 1) DBDM in general; 2) analyzing student achievement data with the student monitoring system; 3 ) setting goals based on data; 4) collaboration during the DBDM process (see Appendix G).

A data literacy test was administered at T1 and T3 to gain insight into the initial data literacy of school staff and changes resulting from the two-years of DBDM implementation. Three versions of this test were developed since three SMSs are used in Dutch primary schools. The questionnaire included 15 general data literacy items, and, depending on the SMS-software the school used, 10 to 15 system specific interpretation items (see Appendix B).

To measure teaching quality, a student questionnaire was developed with 36 items divided over five subscales: safe classroom climate, classroom management, clear instruction, goal-orientation, and challenging students (see Appendix C). All questions were scored on a five-point Likert scale, ranging from 'no, never' to 'yes, always'.

Note that educators' DBDM relevant knowledge and skills were measured by means of a data literacy test and a teaching quality survey. Within the scope of this study it was unfortunately impossible to collect data on educators' knowledge and skills regarding the setting of SMART and challenging goals and determining strategies for goal accomplishment.

\section{Data Analysis}

See appendix $\mathrm{H}$ for each of the sources used for this study presents the analyses that were conducted for computing the values used. In most cases the analyses were performed for all schools that participated in the DBDM intervention. A detailed description of these analyses can be found in chapters 2 and 3 (student achievement outcomes), chapter 4 (data literacy), chapter 5 (teaching quality), chapter 6 (educational school leadership), chapter 7 (data culture) and chapter 8 (collaboration). In this article, the outcomes described in appendix $\mathrm{H}$ were used to provide an in-depth picture of the selected schools.

\section{Results}

To answer our first research question, about the factors that school leaders and/or trainers perceived as having promoted or hindered DBDM implementation the results from the interviews with school leaders and trainer reports are presented for each factor studied.

Next, in order to answer the second research question, concerning the differences between 'no-effect schools' and 'strong-effect schools,' the results from the surveys are presented.

First, the results on the educator characteristics are presented, followed by the results on the school organizational characteristics. Two factors, not included in our theoretical framework but considered important by the school leader and/or the trainer, are presented at the end of this section.

\section{Educator Characteristics}

Educators' knowledge (data literacy). Based on the storylines plotted by the school leaders, we found that especially the first four meetings of the intervention resulted in 
a peak in the implementation process. In these meetings, the basic knowledge and skills for DBDM were acquired. Teachers gained insights into the possibilities of the student monitoring system, learned to set SMART goals, and how to formulate these goals and the strategies, to achieve the goals in an instructional plan. ${ }^{1}$ Sixteen out of twenty schools reported that these meetings were very beneficial for DBDM implementation in their school. No differences were found between strong-effect and no-effect schools based on qualitative data. For example, the school leader of NE20 said:

"After meeting 1.1 there was a peak, we had no idea what the possibilities of our student monitoring system were, and teachers were very enthusiastic when they found out they could for example graph their students' achievement growth".

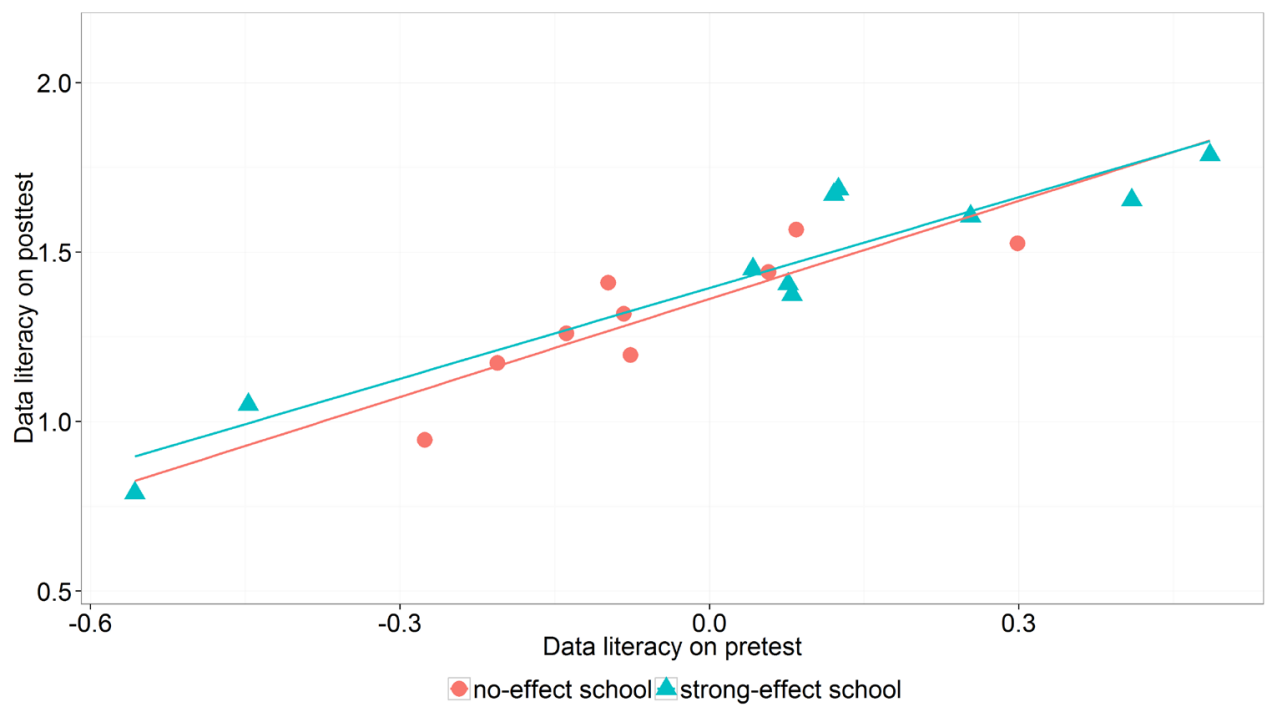

Figure 2. Schools' average data literacy scores on the pretest plotted against posttest scores

Based on the data from the data literacy test, no differences were found between no-effect schools and strong-effect schools in terms of their data literacy growth. In Figure 3, teachers' pretest data literacy scores (averages per school) are plotted against their posttest data literacy scores (averages per school), and a comparison is made between no-effect schools and strong-effect schools. As can be seen in Figure 3, in both groups data literacy strongly improved during the intervention.

In sum, although school leaders reported data literacy growth as a factor promoting DBDM implementation, examination of the quantitative survey data revealed no relationships between educators' data literacy growth and DBDM implementation success.

Teaching quality. Based on trainer reports, it appeared that teaching quality was an important factor for successful DBDM implementation. Moreover, a clear difference

\footnotetext{
1 Note that school leaders and trainers reported the importance of DBDM knowledge and skills regarding goalsetting and regarding choosing strategies, however survey data was only available for data literacy skills and teaching quality, and thus the importance of goal-setting skills and strategy choice skills could not be confirmed with the survey data in this study.
} 
Table 3. Means and standard deviations per teaching quality scale in strong-effect schools $(\mathrm{N}=32)$ and no-effect schools $(\mathrm{N}=21)$ for Focus III schools

\begin{tabular}{|c|c|c|c|c|c|c|c|c|}
\hline & \multicolumn{4}{|c|}{$\begin{array}{l}\frac{\text { Teachers in }}{\text { strong-effect schools }} \\
\underline{\text { s. }}\end{array}$} & \multicolumn{4}{|c|}{$\begin{array}{c}\text { Teachers in } \\
\text { no-effect school }\end{array}$} \\
\hline & \multicolumn{2}{|c|}{ Pre } & \multicolumn{2}{|c|}{ Post } & \multicolumn{2}{|c|}{ Pre } & \multicolumn{2}{|c|}{ Post } \\
\hline & M & (SD) & M & (SD) & M & (SD) & M & (SD) \\
\hline F1: creating a safe climate & .13 & $(.49)$ & .11 & $(.47)$ & .04 & $(.50)$ & .04 & $(.40)$ \\
\hline F2: classroom management & .19 & (.64) & .19 & $(.46)$ & .01 & $(.64)$ & .14 & $(.46)$ \\
\hline F3: clear instruction & .19 & $(.40)$ & .11 & $(.31)$ & -.07 & $(.41)$ & -.09 & $(.38)$ \\
\hline F4: challenging students & .28 & $(.48)$ & .55 & $(.37)$ & -.10 & $(.47)$ & .25 & $(.37)$ \\
\hline F5: goal-oriented teaching & .11 & $(.38)$ & .49 & $(.45)$ & -.33 & $(.40)$ & .03 & $(.43)$ \\
\hline
\end{tabular}
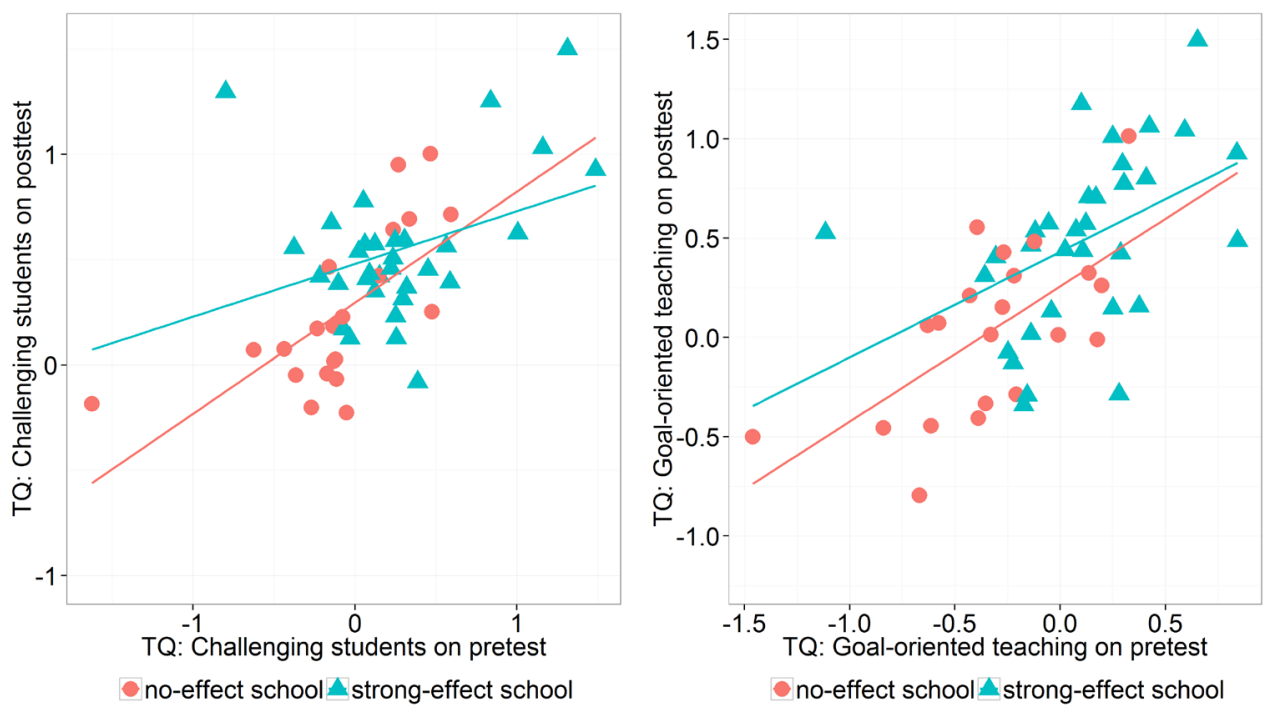

Figure 3. Pretest and posttest scores for the teaching quality scales challenging students and goal-oriented teaching. The black dotted line represents the line of a perfect correlation between pre-scores and post-scores. The vertical black line represents the population average pretest score of zero.

between no-effect and strong-effect schools was mentioned by the trainers. Based on the lesson observation round(s) in the three schools, trainers reported variation in teaching quality between teachers. However, trainers reported that in seven out of ten strong-effect schools overall teaching quality was 'sufficient' to 'excellent'. Moreover, in Focus III, in the second lesson observation round most teachers had improved compared to the first lesson observation round. The trainer of school SE1:

"Compared to the first round of lesson observations, the teachers in this school really improved their teaching practice. They have used the feedback to resolve issues that deserved attention, which I now regard as qualities."

In contrast, in three out of ten no-effect schools trainers reported teaching quality as 
sufficient. In one school the trainer could observe too few lessons to judge teaching quality. Moreover, trainers reported weak to moderate lessons in two no-effect schools. For example, school NE17:

"The lessons I observed were not in line with the instructional plans the teachers had written. Overall, lesson quality was not that bad, but there was considerable room for improvement. First of all, classroom climate, classroom management, and providing clear instruction should be up to standard, before teachers try to implement the more complex instructional strategies they described in their plans".

In four schools trainers observed considerable variation in teaching quality between teachers, which they reported as a hindering factor for implementing DBDM.

Quantitative teaching quality measures, which were only available for the Focus III schools, confirmed trainers' observations. As can be seen in Table 3, at the pretest students rated their teacher, on average, more positively on all five teaching scales in the strong-effect schools than students in the no-effect schools. At the posttest, teachers in the no-effects schools, in the eyes of their students, improved considerably on classroom management, challenging students and goal-oriented teaching. However, teachers in the strong-effects schools still outperformed teachers in the no-effect schools.

The difference between strong-effect schools and no-effect schools is largest for the 'challenging students' and 'goal-oriented teaching' teaching scales. For these two teaching skills, which are especially important for DBDM, teachers in the strong-effect-schools had higher pretest scores. In Figure 5, circles represent teachers in strong-effect schools and triangles represent teachers in no-effect schools. Note that teachers who scored below the dotted line did not improve their score on the posttest. As judged by students, the majority of teachers improved their teaching skills, however teachers in the strong-effect schools showed more improvement compared to teachers in no-effect schools (represented by a higher intercept in Figure 5). Moreover, especially for the factor goal-oriented teaching, prior to the intervention the majority of teachers in the no-effect schools scored below the population average.

To conclude, the importance of teaching quality for DBDM implementation was shown by both the qualitative and quantitative data. Trainers more often reported strong teaching quality in strong-effects schools, and more often weak or varying teaching quality in no-effect schools. Quantitative data showed that although teaching quality improved in no-effect schools and strong-effect schools, teaching quality in no-effect schools still lagged behind teaching quality in strong-effect schools.

Attitude. In seven strong-effect schools and in four no-effect schools, trainers identified positive team attitudes as a promoting factor. Such a positive attitude was characterized as a highly motivated team that was eager to learn and change their practice based on the feedback they received from the trainer. These teams recognized the usefulness of the intervention. As trainers reported such a positive attitude in both groups of schools, a positive attitude does not seem to cause differential intervention effects. However, a negative attitude towards DBDM implementation seems to cause differences between strong-effect and no-effect schools. Trainers reported negative attitudes towards the intervention or 

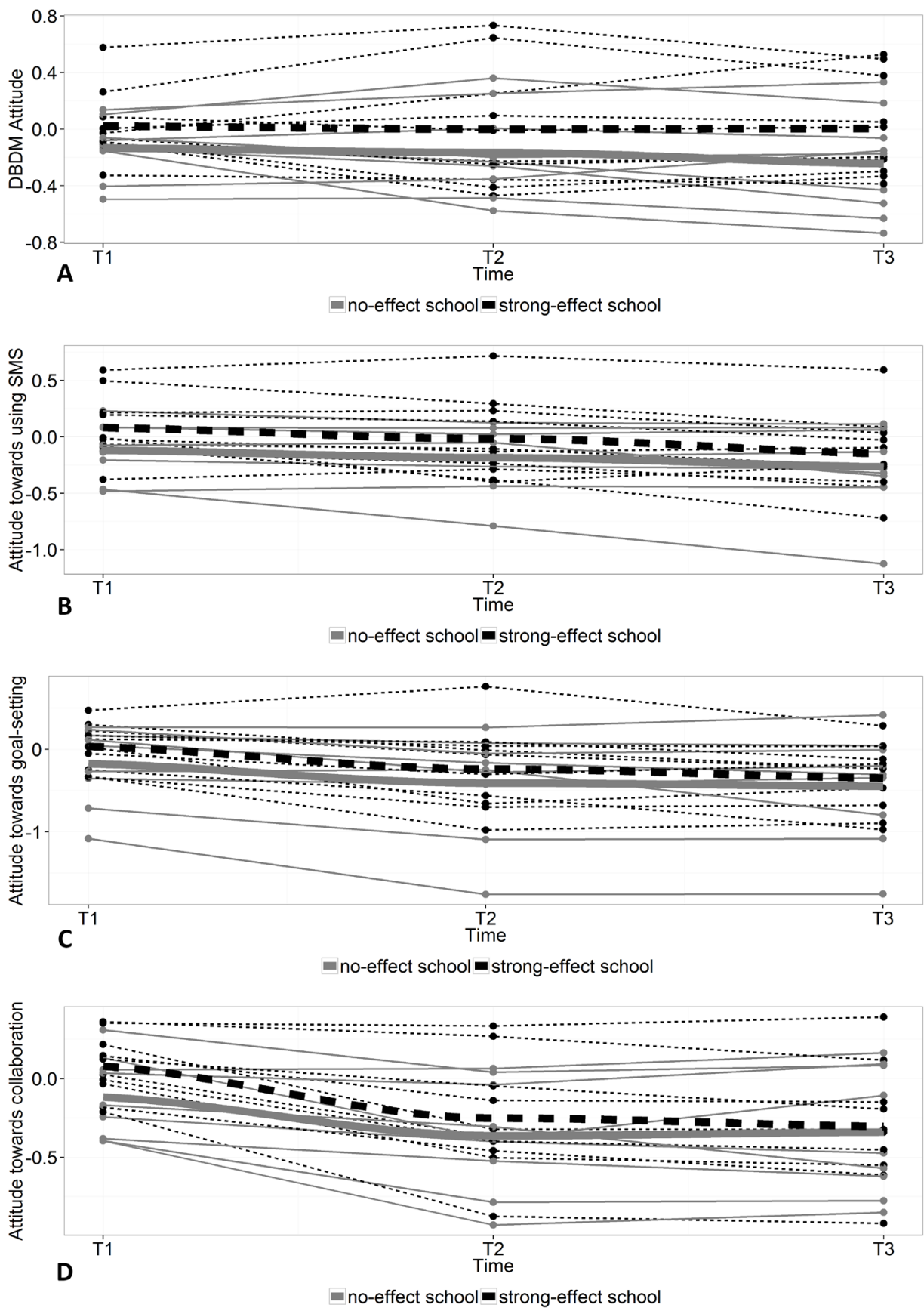

Figure 4. Schools' attitudes towards a) DBDM in general; b) analyzing student achievement data with the student monitoring system; c) setting goals based on data; and d) collaboration during the DBDM process. The bold dotted line represents the average strong-effect school attitude, the bold solid line represents the average no-effect school attitude 
towards DBDM as a hindrance to implementation. In the no-effect schools, teachers were more often critical towards DBDM compared to teachers in strong-effect schools. In seven out of ten no-effect schools the project trainers reported that, at some point during the intervention, they experienced a negative attitude and resistance towards DBDM. In contrast, trainers reported negative attitudes in three out of ten strong-effect schools. Reports from the trainer revealed that this negative attitude towards DBDM in the no-effect schools had multiple reasons. Some schools had a negative attitude at the start of the intervention, which was mostly caused by a lack of agreement within the team to participate in the DBDM intervention. For example, the school leader at school NE14:

"Participation in the intervention was demanded by the school board, therefore, the school team was initially unmotivated".

Other schools started as motivated, but became demotivated in the second intervention year. For example on school NE17, the trainer reported:

"During the first year, the teachers were very dedicated and enthusiastic. They were critical but motivated, and worked hard on implementing DBDM. During the second year, there was a lot of discussion but no decisions were made. A lack of leadership and clear agreements led to diversity in, for example, the instructional plans and the depth of data analysis, and eventually to a stagnation in DBDM implementation".

Furthermore, in some schools a negative attitude towards DBDM and the DBDM intervention was mentioned as a result of 'confusion' within the team. This, for example, applies to school NE11, in which the information presented in the first meetings was not in line with how the school already worked in terms of DBDM.

Although not all members in the no-effects schools had a negative attitude towards DBDM project, trainers reported that if a team displayed a negative attitude, DBDM implementation within the whole school could be delayed. For instance, the trainer of school NE20:

"All teachers attended all meetings, but only the teachers in the higher grades participated actively in the meetings. Kindergarten teachers were not motivated and were just there because they felt they had to. I think the intervention therefore had an effect on individual teachers, but not on the school as a whole."

In contrast, in those strong-effect schools (three out of ten) in which negative attitudes were mentioned, trainers only experienced this at the start of the intervention, for example in school SE07:

"During the first four meetings, the team was not motivated and teachers were afraid DBDM would only imply a lot of paperwork. However, after meeting 1.5 in which student achievement was discussed for the first time, they became aware of the benefits of DBDM." 
Our quantitative data supplement the findings from the trainer reports. Four scales were used to measure attitude. The first scale concerned participants' general attitude towards DBDM. As can be seen in Figure 4a, large variation between the 20 schools was found. On average, team members in the no-effect schools started with a less positive attitude towards DBDM. Additionally, the average attitude towards DBDM decreased in the no-effect schools, while the attitude remained positive in strong-effect schools.

Aside from the general attitude scale, the attitude towards specific DBDM components (analyzing student achievement data with the student monitoring system; setting goals based on data; and collaboration during the DBDM process) was measured. Figures $4 b, 4 c$, and $4 d$ show that, for all three scales, the same trend was found as in the general attitude scale: great variability between schools and in general a more positive attitude in strong-effect schools. As can be seen in Figure 4, in both groups of schools the attitude slightly declined over time. Remarkably this decrease was stronger for the strong-effect schools.

In sum, our findings from both qualitative data as well as quantitative data show that teachers' attitudes towards DBDM seem to be related to DBDM implementation success. In particular, trainers more frequently reported a negative attitude towards DBDM as a hindering factor in DBDM implementation in no-effect schools compared to strong-effect schools. According to the trainers, staffs' negative attitudes encountered at the start of the intervention in three strong-effect schools disappeared during the intervention, when teachers experienced the benefits from DBDM. This was not the case in the no-effect schools. Quantitative data confirmed the differences between attitude towards DBDM in strong-effect and no-effect schools, although it should be noted that the average attitude between schools varied considerably.

\section{School Organizational Characteristics}

Educational leadership for DBDM. In both groups of schools project trainers reported that the school leader was either a promoting or hindering factor during the implementation of DBDM. A school leader who was not involved in the innovation process was regarded as a hindering factor, in most of these cases the school leaders delegated the responsibility of leading the DBDM process to their academic coach. For example in school SE5, the trainer reported:

"In this school, the academic coach was key to the entire process of change. The school leader and deputy school leader showed a lack of knowledge with regards to the student monitoring system, and expected the academic coach to analyze and interpret data at the school level."

Other reasons for mentioning the school leader as a hindering factor for DBDM implementation were: weak communication within the management team or a school leader without authority. Finally, trainers felt that overprotective school leaders delayed the implementation of DBDM, by skipping, or postponing intervention parts because (s)he was afraid that staff members were too busy to work on all DBDM components.

School leaders who promoted the implementation of DBDM were described by the trainer as 'involved', knowledgeable about the needs of their team members, and communicative about the added value of DBDM. According to the trainers, such school leaders were particularly important during the meetings in which student achievement was 
discussed with the whole team (meetings 1.5 and meeting 1.7 in the first year, and meetings 2.3 and 2.5 in the second year). In this meeting, the school leader presented the student achievement results of the school. A trainer on school SE10:

"This school really celebrated success. The school leader brought a cake to celebrate the raise in student achievement and complimented teachers for the good job they had done. He made sure he attributed the success to the teachers and the way they implemented DBDM".

Furthermore, during these meetings strong school leaders made clear agreements with their team members and ensured that these agreements were met.

Quantitative data revealed no meaningful differences between school leaders in strong-effect schools compared to school leaders in no-effect schools. School leaders' background characteristics were similar. In both groups, there were six male school leaders and four females, the majority of them in the age group 51-60. Most school leaders had a Master's degree. In all schools selected for this study, the same person fulfilled the formal role of school leader during the entire intervention.

Table 4 presents the assigned school leader classes for T1, T2 and T3, where classes of leadership for DBDM are ordered from the least DBDM oriented (SL1) to the most DBDM oriented school leader (SL5). No remarkable differences with regards to assigned school leadership class were found between strong-effect and no-effect schools. Additionally, in the strong-effect schools, four school leaders were assigned to the same leadership class at the start four school leaders moved to a higher class at the end of the intervention, and one school leader moved to a lower class. In the no-effect schools, three schools were assigned the same leadership class, while six school leaders moved to a higher class, and one to a lower class.

Table 4. Assigned school leader classes for T1, T2 and T3

\begin{tabular}{|c|c|c|c|c|c|c|}
\hline & \multicolumn{3}{|c|}{ Strong-effect schools } & \multicolumn{3}{|c|}{ No-effect schools } \\
\hline & \multicolumn{3}{|c|}{$(N=10)$} & \multicolumn{3}{|c|}{$(\mathrm{N}=10)$} \\
\hline & $\mathrm{T} 1$ & T2 & T3 & T1 & $\mathrm{T} 2$ & T3 \\
\hline SL 1 & 1 & 1 & 1 & 1 & 2 & 1 \\
\hline SL 2 & 5 & 3 & 2 & 4 & 2 & 3 \\
\hline SL 3 & 2 & 4 & 3 & 4 & 2 & 2 \\
\hline SL 4 & 0 & 0 & 2 & 1 & 1 & 1 \\
\hline SL 5 & 2 & 2 & 2 & 0 & 2 & 3 \\
\hline
\end{tabular}

To conclude, since trainers emphasized the importance of strong school leadership for DBDM implementation, one would expect strong leaders in strong-effect schools and weaker leaders in no-effect schools. This was not reflected by the quantitative data, which revealed that no clear leadership differences were found between strong effect and no-effect schools.

Data culture. In the interviews with the school leaders and the trainer reports 'data culture' was not often mentioned as a promoting or hindering factor. This may be have been caused by the fact that data culture is related to educators' DBDM attitudes, which was mentioned by them. Moreover, it is a scientific concept not commonly used by educators. 

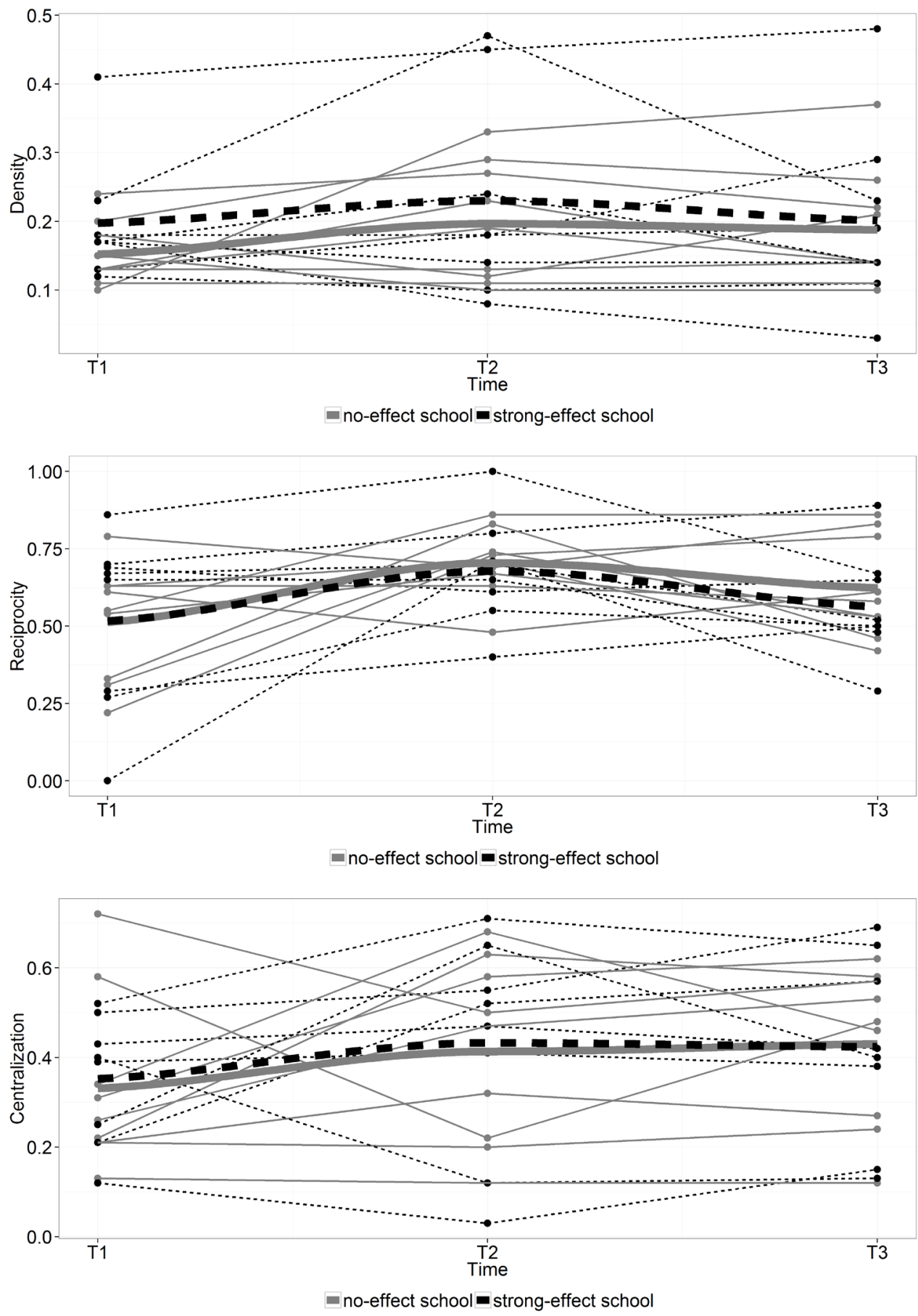

Figure 5. Density, reciprocity and centralization measures per school, per time point for the 'discussing student achievement' network; the bold dotted line represents the average, strongeffect school attitude; the bold solid line represents the average no-effect school attitude 
In Table 5, the number of schools per assigned data culture class is presented for each time point. Data culture class 1 (DC1) can be regarded as really low, whereas data culture class 5 (DC5) represents a strong data-oriented school culture. At T1 the majority of no-effect schools was assigned a low data culture class, while the strong-effect-schools were more often assigned as higher data culture classes. All strong-effect schools were assigned a higher data culture class at the end of the intervention than at the start of the intervention. Six of the no-effect schools moved to a higher data culture class and the other four schools were assigned the same data culture class remained the same. At the end of the intervention, more strong-effect schools were assigned high data culture classes compared to no-effect schools.

Table 5. Assigned data culture classes for T1, T2 and T3

\begin{tabular}{|c|c|c|c|c|c|c|}
\hline & \multicolumn{3}{|c|}{ Strong-effect schools } & \multicolumn{3}{|c|}{ No-effect schools } \\
\hline & \multicolumn{3}{|c|}{$(\mathrm{N}=10)$} & \multicolumn{3}{|c|}{$(\mathrm{N}=10)$} \\
\hline & T1 & T2 & T3 & T1 & $\mathrm{T} 2$ & T3 \\
\hline$\overline{\mathrm{DC} 1}$ & 2 & 0 & 0 & 3 & 0 & 0 \\
\hline DC 2 & 3 & 2 & 0 & 5 & 5 & 2 \\
\hline DC 3 & 4 & 1 & 2 & 0 & 1 & 3 \\
\hline DC 4 & 1 & 4 & 6 & 2 & 3 & 5 \\
\hline DC 5 & 0 & 3 & 2 & 0 & 0 & 0 \\
\hline
\end{tabular}

To conclude, although trainers and school leaders did not mentioned 'data culture' as a factor promoting or hindering DBDM implementation, the survey data revealed that a data and achievement oriented school culture seems to be related with successful DBDM implementation. At the start of the intervention, strong-effect schools were assigned to higher data-culture classes compared to no-effect schools. Although the majority of the schools moved to a higher data culture class during the intervention, more strong-effect schools were assigned to high data culture classes at the end of the intervention compared to no-effect schools. It can thus be concluded that a data- and achievement oriented school culture is positively related to DBDM implementation.

Collaboration. In the interviews with school leaders, in both strong-effect and in noeffect schools, 'collaboration' was mentioned as a factor promoting DBDM implementation. Such collaboration included discussing student achievement in upper, middle and lower grades, and in parallel classes (including searching for explanations for high or low student outcomes), sharing experiences and best practices, and discussing instructional plans with each other. Furthermore, openness, trust and reflection skills as perceived by school leaders were prerequisites for quality collaboration. School leaders reported that, as a result of the DBDM intervention, collaboration among their staff increased. However, schools also struggled with providing time for collaboration, for example the trainer of school NE20:

"There proves to be more collaboration between teachers, especially after meeting 1.6. The younger teachers share their instructional plans with the rest of the team and help other teachers. Due to illness however, the school leader and the academic coach have not yet been able to accomplish teacher peer coaching in classrooms."

To quantitatively study collaboration, schools' social networks were studied. The 'achievement 
network', the 'goal-network', and the 'instructional strategies network' were examined based on the matched dataset, imply that only those respondents who were present at T1, T2, and T3 were included in the network analyses. The density (the concentration of relationships in a social network), reciprocity (the extent to which relationships in a network are reciprocal), and centralization (the degree to which a network is centralized around one or more popular actors) of these three network types were calculated for all three time points. This resulted in nine outcome variables per time point (density, reciprocity and centralization at T1, T2 and T3). For two schools (one in the strong-effect group, and one in the no-effect group), network measures were not available, since such measures would be too severely by the small number of respondents (fewer than 5) in these schools. Additionally, for one school (NE19) no network measures were available at T2, so this school was also removed. Figure 6 shows the results for each of the three network measures of the student achievement network. This figure shows strong variation on all three network measures.

At the start of the intervention, $15 \%$ of all possible relationships in the network actually existed (density) in the strong-effect schools whereas the density was $20 \%$ in the noeffect schools. At the end of the intervention, density remained comparable in the no-effect schools (20\%) and had, on average, slightly increased in strong-effect schools (19\%).

No differences were found at the start of the intervention regarding the extent to which relationships in a network were reciprocal.. At the end of the intervention, a small average difference in favor of the strong-effect schools was found, in these schools $62 \%$ of all relationships in the network were reciprocal versus $56 \%$ in the no-effect schools. Similarly, no clear differences were found for centralization, the degree to which a network is centralized around one or more 'popular' actors.

Results for the achievement network are very similar to the findings for the goal network and the instructional strategies network: no significant differences between strongeffect and no-effect schools.

In sum, school leaders emphasized the importance of collaboration within their team for the successful implementation of DBDM. However, this was not confirmed by the quantitative data. Although some small differences were found between strong-effect and no-effect schools, variation between schools' networks with each group was so strong that no clear pattern could be discerned.

\section{Two Additional Factors Mentioned by School Leaders and Trainers}

In addition to the factors that were identified as influential to the implementation of DBDM in literature, school leaders and trainers also indicated two additional factors that they perceived as influential. These factors consisted of the presence of a (supportive) academic coach and pressure from the Inspectorate of education.

The academic coach. In both no-effect (five out of ten) and strong-effect schools (seven out of ten), trainers felt DBDM implementation benefitted from a supportive academic coach. Such an academic coach was characterized by the trainers as a person knowledgeable regarding DBDM and dedicated to its implementation. Supportive academic coaches were also critical towards the intervention, making sure it would be consistent with the school's procedures. According to the trainers, such academic coaches were even greater driving forces of DBDM implementation than school leaders.

The trainers of five out of ten no-effect schools reported issues with the academic 
coach hindering DBDM implementation. In these schools the academic coach did not have enough time to fulfill his role, lacked the required knowledge and skills to support colleagues in the DBDM process, and one school lacked an academic coach. In strong-effect schools, no problems with the academic coach were reported.

Inspectorate. Three out of ten strong-effect schools had been reported as a 'weak school' by the Dutch Inspectorate of education. In these schools the pressure from the Inspectorate was mentioned as both hindering and promoting. On the one hand, this pressure increased motivation for improvement. On the other hand, the work pressure was high and overly stressful. None of the no-effect schools reported pressure from the Inspectorate as promoting or hindering for DBDM implementation.

\section{Conclusions and Discussion}

The main purpose of this study was to explore why identical DBDM interventions led to improved student achievement in some schools, while not affecting student achievement in other schools. Previous research (chapter 2 and chapter 3 ) into the impact of the Focus intervention has revealed that school-SES and schools' intervention trajectories partly explain intervention effect differences. Schools with a high percentage of students from lowSES backgrounds and schools that focused on mathematics during the entire intervention period achieved the most achievement growth. The current study tried to explain which other factors influenced the differences in intervention effects between schools.

Based on the DBDM literature, educator and school organizational features were selected that are considered to promote or hinder DBDM implementation. Ten schools with strong DBDM intervention effects on student achievement were compared with ten schools with no intervention effects. The results from interviews with school leaders and school reports from trainers were studied to answer our first research question, concerning the factors school leaders and trainers reported as promoting and hindering DBDM implementation within their schools. We were curious as to whether the factors mentioned by trainers and school leaders were similar to the ones reported in the DBDM literature. Findings showed that this was the case, all the factors mentioned in the DBDM literature and in our view most relevant for our topic (educators' knowledge and skills, their attitudes towards DBDM, school leadership, data culture, and school internal collaboration), were also mentioned by the school leaders and trainers. Moreover, two other factors were also mentioned by the school leader and the trainers, the role of the academic coach and the pressure from the Inspectorate of education. Some school leaders reported this pressure as motivational whereas others indicated that this pressure led to high pressure and stress on their staff members.

To find out what differentiates schools that benefitted most from the intervention from those that did not benefit from the intervention (research question 2), survey data was examined. Based on an examination of the data, three factors proved to be directly associated with strong effects on student achievement growth: educators' DBDM attitudes, a school's data culture, and the teaching quality of the teachers of a school.

In the following section, these three factors will be described after which we reflect on the other factors studied. Subsequently, some limitations of our study will be discussed and topics for future research will be reflected upon. Finally, implications of our findings for school practices will be given. 


\section{Factors Explaining Differences Between Strong-Effect and No-Effect Schools}

At the start of this study we formulated the expectation that strong-effect schools, in comparison with no-effect schools, would be characterized by teachers with higher teaching quality, staff who are more positive towards DBDM, and a school culture that was more dataoriented and achievement oriented. This study confirms these expectations.

Findings from the exploration of the survey data showed that prior to the intervention schools already differed in terms of the quality of their teachers, their DBDM attitude, and data culture. At the start of the intervention educators' attitudes towards DBDM were lower, the culture was less achievement oriented, and teachers on average were rated lower by their students on all teaching scales in no-effect schools when compared to strong-effect schools.

During the intervention, educators' attitudes either remained stable or became a little less positive in both groups of schools. Moreover, although a more achievement oriented data culture developed during the course of the intervention in both groups, the initial difference between schools with regards to data culture still existed at the end of the intervention. A similar trend was found for teaching quality. Although teachers in the no-effect schools showed improvement on all teaching quality scales, except for clear instruction, they did not even reach the starting levels of the teachers in the strong-effect schools.

These findings from the survey data were confirmed by the trainer reports. Trainers also reported stronger teaching quality behavior in strong-effect schools compared to noeffect schools. Moreover, they more often faced a negative attitude from staff members in no-effect schools. Data culture was not mentioned in trainer reports or school leader interviews, probably because such a theoretical construct is infrequently used by educators.

\section{Factors Not Explaining Differences Between Both School Groups}

Besides a stronger teaching quality in strong-effect schools, a more positive attitude of staff members and a stronger data culture, we formulated expectations with regards to the impact of staff data literacy, school leadership, and collaboration within the team on DBDM implementation. Exploration of the quantitative data did not confirm these assumptions.

First, a difference was expected in educators' data literacy between no-effect schools and strong-effect schools at the end of the intervention, as data literacy is regarded as a precondition for DBDM in literature, and thus a precondition for improved student outcomes (Mandinach \& Gummer, 2013a; Mandinach et al., 2006). School leaders did mention improved knowledge and skills as a valuable result of the intervention. However, results showed that data literacy improved to the same extent in both groups of schools and therefore it does not explain differences between schools in intervention effects on student achievement.

Second, we hypothesized that school leaders in strong-effect schools would be perceived as more achievement oriented than school leaders in no-effect schools. Trainers reported the school leader to be important for the DBDM process within the school, however, trainers in both strong-effect schools and in no-effect schools reported stronger and weaker school leaders. Moreover, our findings from the teacher questionnaire did not confirm differences in school leadership classes between strong- and no-effect schools.

Third, it was expected that collaboration patterns would be stronger in strong-effect schools. In the interviews, school leaders of both strong- and no-effect schools indicated that strong collaboration, characterized by discussing student achievement data, sharing experiences and best practices, promotes the DBDM-implementation process. Based on the data concerning the school social networks, no clear conclusions could be drawn regarding 
their explanatory role as the network measures varied from each other regardless of the school group.

\section{Putting It All Together}

How can we interpret our overall findings? As Desimone (2009) argued, in order to improve student outcomes by means of an intervention, a change in teachers' classroom behavior is necessary. The effect of teaching quality on improved student outcomes thus seems logical. The other two factors that explain differences between schools are factors related to attitudes and values regarding perceptions of what is important. Whether or not staff members are willing to change their day to day practice and invest in an intervention like DBDM is dependent on their attitudes towards that intervention and the degree to which the intervention matches the school culture. In schools with an achievement oriented and data-oriented school culture, teachers will be more motivated to work in a data-based way.

How can the finding that school leaders and trainers, in line with the DBDM literature, emphasize the importance of data literacy, school leadership and collaboration, while our comparison of strong- and no-effect schools revealed that these factors did not explain differences in intervention effects between schools be explained?

Although our findings provide evidence that educators' data literacy in all schools considerably increased, it did not explain differences between schools. A possible explanation for our findings may be that data literacy a necessary precondition rather than a sufficient precondition for successful DBDM implementation. A teacher may be perfectly capable of interpreting and analyzing data, but improved student outcomes are not likely to occur if (s) he does not know how to change classroom practice based on the analyses.

The importance of the school leaders as educational leaders is emphasized by numerous authors (Hallinger \& Heck, 2011; Ikemoto \& Marsh, 2007; Levin \& Datnow, 2012b; Schildkamp \& Lai, 2013). A school leader is supposed to promote DBDM implementation by fulfilling practical preconditions for DBDM (e.g. buying a proper student monitoring system, and providing time for DBDM activities and collaboration), by promoting and facilitating data use and demonstrating a strong DBDM vision. In a previous study (chapter 7), we found that a strong data culture and DBDM oriented school leadership were positively associated. Seemingly, the impact of a school's data-culture on DBDM-effects is stronger than that of school leadership, perhaps other staff (e.g. the academic coach) also contributed to the data culture.

Finally, the measures used to study collaboration patterns in schools showed large variability within the schools. This made it impossible to explain any relations between strong- and no-effect schools.

Overall, we do not claim that data literacy, school leadership and internal collaboration within schools are unimportant for DBDM but rather that these factors did not explain differences in DBDM implementation in our schools.

\section{Limitations of the Study}

The study which we report here is an exploratory study and meant to investigate which factors influenced the success of DBDM interventions in depth. There are a number of limitations to this exploratory study. First, the 20 schools included consisted of ten schools with the strongest intervention-and weakest intervention effects out of 101 schools, by doing so we focused on the outliers in the original sample. In particular, the comparison of the characteristics of the strong-effect schools with the Dutch national primary school population 
revealed that these schools were not representative, as these schools were more frequently located in (sub) urban areas and low-SES schools were considerably overrepresented. Thus, our selection procedure enabled us to make a comparison between schools but also limits the generalizability of our findings. The sample was also small. The sample size was chosen to be able to conduct an exploratory study that would enable the exploration of those factors that differentiate between strong-effect schools and no-effect schools. We acknowledge that a large-scale follow-up study, with a larger and more representative sample is needed for more generalizable conclusions.

Moreover, schools were selected based on their 'intervention effect' which was calculated by comparing student achievement growth during the two intervention years with student achievement growth during the two years before the intervention (see also chapters 2 and 3). It should be noted that the no-effect schools were not, by definition, low-achieving schools. Some of them were high-achieving schools with stable performance during the DBDM intervention. In contrast, the majority of strong-effect schools were initially low-achieving schools and thus more growth was possible in these schools. It could be that no-effect schools already performed so well that it proved to be more difficult to further improve their performance.

In order to gain more insight into the effects of DBDM, future research aimed at examining differences between schools in which DBDM was implemented successfully versus unsuccessfully s should use a DBDM-measure, rather than the product of DBDM for selecting the schools to be studied. For example, Van Geel, Teunis and Visscher (2016) used trainers' overall grade (ranging from 1 to 10), representing the degree to which DBDM had been embedded in a school. In this way it was possible to rank schools, where high scores reflected schools more successful in implementing DBDM.

\section{Future Research and Recommendations for Practice}

As this study was aimed at 'unpacking the package' of promoting and hindering DBDM factors, factors were separately assumed to influence DBDM implementation. For each variable a separate comparison was made between no-effect schools and strong-effect schools. However, as schools are complex organizations, in which variables are intertwined with each other, it is plausible that the way in which various variables interplay with each other causes differences in DBDM implementation. It may also be that some factors compensate for others, e.g., a school leader who is not very achievement oriented may not be a problem in a school in which the academic coach is the driving force for DBDM. Therefore, in future studies the investigation of (various) clusters of variables by means of cluster analysis is recommended. This way, combinations of factors can be studied and related to DBDM implementation success.

Data-based decision making encompasses more than analyzing data. As Kaufman (2014) states: "While identifying and analyzing data lays the groundwork for impactful improvements to student learning, the resulting actions and progress monitoring will ultimately determine the efficacy of DDDM efforts" (p. 341). In line with this statement, we decomposed DBDM into four components in our conceptual model: analyzing and evaluating data, setting SMART and challenging goals, choosing strategies to accomplish the goals, and executing the chosen strategies for goal accomplishment.

The survey data provided information about teachers' knowledge and skills with regards to analyzing data (the first DBDM-component) and with respect to the execution of DBDM within the classroom as perceived by students (the fourth component). Based on 
our findings, we argue that the success of DBDM implementation is very dependent on the quality of the last component of the DBDM cycle. This entails that teachers are required to be able to differentiate their instruction in accordance with variation in student progress and needs (Visscher, 2015). Providing differentiated instruction is something teachers have much difficulty with. According to the Dutch Inspectorate of Education, teachers in about half of all Dutch elementary schools do not differentiate instruction well enough to meet the needs of all students (Inspectie van het Onderwijs, 2014).

Our survey data did not provide information about teachers' abilities to set SMART and challenging goals (the second DBDM component) and their ability to determine strategies for goal-accomplishment (the third DBDM component). In future studies, information about these two components should also be collected. In the current study, we could not provide any evidence of the impact of these two aspects of DBDM on student achievement growth. However, we expect that the third component is especially important for the execution of DBDM in the classroom. Improved student outcomes are likely when teachers are able to deliberately select the strategy that meets students' needs.

Currently DBDM innovation initiatives are often focused on skills that teachers need to master outside of the classroom, such as the knowledge and skills for analyzing data (Mandinach, 2012; Marsh, 2012). The assumption is that, as a result of developing these skills outside of the classroom, changes in teachers' classroom behavior will follow (Desimone, 2009). This study clearly shows the importance of teaching skills. Therefore, when developing a DBDM school reform, we recommend sufficient attention is paid to these teaching skills within the classroom. For example, by including coaching sessions and classroom observations as a basis for providing teachers with feedback on their teaching skills (Van der Scheer \& Visscher, 2014). 


\section{General Conclusions and Discussion}


In the Netherlands, similarly to the international context, there is a growing attention for the use of data to base (instructional) decisions on (Inspectie van het Onderwijs, 2010; Visscher \& Ehren, 2011). The underlying assumption is that data-based decision making (DBDM) enhances student achievement (Coburn \& Turner, 2012). The idea of using student achievement data for evaluating student progress, providing tailored instruction, and developing strategies for maximizing student outcomes seems straightforward. However, the amount of empirical evidence on the effectiveness of DBDM interventions is limited. Aside from being scarce, the studies are also inconclusive, as some studies show improved student achievement (Campbell \& Levin, 2008; Carlson, Borman, \& Robinson, 2011a; Konstantopoulos, Miller, \& van der Ploeg, 2013; Lai, Mcnaughton, Amituanaitoloa, Turner, \& Hsiao, 2009; May \& Robinson, 2007), whereas others reveal no effects on student outcomes (Henderson, Petrosiono, Guckenburg, \& Hamilton, 2007; Quint, Sepanik, \& Smith, 2008; Ritzema, 2015). Therefore, there is a need for more research on how data use can reach its full potential (Kaufman, Graham, Picciano, Popham, \& Wiley, 2014). In this dissertation, the effects of a DBDM intervention ("The Focus intervention") were evaluated. The DBDM intervention was unique in two ways. First, the content of the intervention included the whole DBDM package rather than one or two isolated components. The majority of DBDM interventions are primarily aimed at improving the evaluation and analysis of data (Ikemoto \& Marsh, 2007; Marsh, Pane, \& Hamilton, 2006; Oláh, Lawrence, \& Riggan, 2010). DBDM encompasses more (Kaufman et al., 2014), as such the intervention in this dissertation included all components of DBDM: evaluation and analyzing results, setting SMART and challenging goals, determining a strategy for goal accomplishment, and executing this strategy. Second, the whole school team participated in this intervention rather than few team members or the school leader. Consequently, DBDM was implemented throughout the whole school organization and all teachers were involved and influenced by the training. This is important because teachers are considered to influence student outcomes the most (Darling-Hammond, 2000; Hattie, 2009; Kaufman et al., 2014; Nye, Konstantopoulos, \& Hedges, 2004).

It was expected that student outcomes for mathematics and spelling would improve as a result of the two-year DBDM intervention. In the first two studies of this dissertation, this hypothesis was put to the test.

In this project, we not only focused on the product of DBDM (i.e. improved student achievement) but also looked into school organizational factors such as school leadership, school data culture, and school collaboration patterns that influence the DBDMimplementation process.

Educator characteristics such as data literacy, teaching quality, and the attitude towards DBDM identified as influential to DBDM-implementation (Ikemoto \& Marsh, 2007; Mandinach, 2012; Schildkamp \& Lai, 2013; Schildkamp \& Poortman, 2015; Visscher \& Ehren, 2011) were also examined. In the DBDM literature these factors usually are considered stable during an intervention; studies examining whether these factors can be influenced and improved by means of a DBDM intervention are uncommon. Therefore, we also studied the development of these factors throughout the intervention. In chapters 4 to 8 , changes in educator and school organizational characteristics were studied to better understand how a DBDM intervention can influence these factors. In the final study (chapter 9), it was examined whether (the development of) these characteristics can explain variation in the intervention effects (in terms of student achievement) between schools.

In sum, this dissertation contributes to the knowledge base of DBDM by studying 
the effects of a DBDM intervention on student achievement over time, but also by investigating educator and school organizational characteristics before, during, and after the implementation of DBDM, and how variation in the development in these educator and school organizational factors explains variance in DBDM intervention effects on student achievement.

In this final chapter, the results of this dissertation as presented in the preceding chapters are first summarized. Next, the contributions of this study to the DBDM knowledge base and its methodological contributions are discussed. Finally, recommendations for future research and implications for practice are presented.

\section{Student Outcomes}

In the first and second study (chapters 2 and 3), the effects of the intervention on student achievement growth were investigated for both projects separately. An overall intervention effect was found for both Focus II (on math achievement, as described in chapter 2) and Focus III (on math achievement as well as on student achievement for spelling, chapter 3). We expected the intervention effects would differ across schools; therefore, a large sample of schools was selected to participate in the intervention. Our hypothesis was confirmed, the intervention effects differed between schools. This was partially explained by student-level and school-level characteristics, such as socio-economic status (SES) (cf. chapter 2 and 3), and by intervention trajectory (in chapter 3).

The question as to which factors contributed to the intervention's effectiveness remained. In order to gain insight into (changes in) the educator and school organizational characteristics assumed to be associated with the successful implementation of DBDM, we first investigated each of those characteristics separately before, during, and after the intervention. Below, the results of each of those studies are summarized. In the last study (chapter 9), all these characteristics were compared across ten schools with the largest and ten schools with the smallest intervention effects. The results of that study will also be summarized here.

\section{Educator Characteristics}

Educator's knowledge and skills are assumed to be important factors influencing the implementation of DBDM. Changes at the educator level were investigated for two educator characteristics: data literacy (study 3) and teaching quality (study 4).

\section{Data Literacy}

In Study 3 (chapter 4), changes in educators' data literacy were investigated. Because of the central role of a student monitoring system in this intervention, the following data literacy definition was used: knowing what kinds of analyses can and cannot be done with the system, and correctly interpreting graphs, tables, and other data representations. During the intervention, educators acquired knowledge and skills regarding assessment and data during meetings on tests, scores, and analyses. Furthermore, intervention participants were taught how to use the student monitoring system software and how to interpret the output. Educators were required to directly apply the new knowledge and skills by analyzing the performance data of their own students at least five times during the two intervention years 
and they did so by following an analysis protocol. They received individualized feedback on their analyses, and the trainers devoted time during the project meetings to discuss common interpretation errors with the entire school team.

In order to assess educators' data literacy, we developed three versions of a data literacy test, which were administered as a pretest and a posttest at the start and end of the intervention. To calibrate the test versions, a multivariate multilevel IRT analysis was conducted.

It was hypothesized that educators' data literacy would improve during the intervention, which was clearly supported by the results. Furthermore, educators with a master's degree initially scored higher, however, the 'data literacy gap' based on participants' education level had been closed by the end of the intervention. School leader data literacy at the posttest proved comparable to teacher data literacy, whereas school leaders significantly outperformed teachers on the pretest. Academic coaches remained significantly more data literate than teachers.

\section{Teaching Quality}

The fourth study (chapter 5) was aimed at the skills teachers need to master in the classroom. Five teaching skills were measured: 1) creating a safe classroom climate; 2) effective classroom management; 3) providing clear instruction; 4) challenging students; and 5) teaching in a goaloriented way. The last two skills were considered to be particularly important for ensuring the effective implementation of DBDM. The study was guided by the question: To what extent does teaching quality develop during a DBDM intervention?

For 209 teachers, teaching quality was measured by means of student perceptions at the start of the intervention (pretest), and after having participated in the DBDM intervention for one year (posttest). For the purpose of this study, a pre-post multivariate IRT model was developed which allowed for the estimation of the effects of the explanatory variables on the pre- and posttest at different modeling levels, while controlling for measurement errors at both time points.

It was concluded that after one year of participating in the DBDM intervention, teachers' DBDM-skills had improved significantly, while their basic teaching skills remained stable. Teachers seemingly managed to communicate more clearly to their students what they were learning and why. Furthermore, they also monitored how their students were progressing during the lessons. Moreover, students felt more challenged by their teacher, implying that the teacher encouraged them to do the best they could to a greater extent. Findings revealed that the intervention was especially beneficial for teachers in multi-grade groups.

\section{School Organizational Characteristics}

Three school organizational characteristics, leadership (study 5), data culture (study 6), and collaboration (study 7), were expected to be related to DBDM implementation. Therefore, the DBDM intervention was also aimed at improving these factors.

\section{School Leadership for DBDM}

School leaders are considered essential for the successful implementation of DBDM. Therefore, study 5 (chapter 6) investigated leadership for DBDM before, during, and after the intervention. Leadership for DBDM was measured by means of teacher perceptions and 
analyzed by applying multilevel latent class analysis. Changes in assignments of school leaders to classes were modelled over time using a multi-state modelling techniques.

Results indicated that leadership for DBDM can be improved during an intensive intervention. For schools in which the same person fulfilled the school leader role throughout the intervention, leadership was stable (44\%) or improved (40\%). Stability was primarily found in schools with initial high leadership for DBDM, whereas improvement of leadership for DBDM was most likely in schools with lower initial leadership.

This study shows that participating in an intensive intervention can contribute to the development of leadership for DBDM, although the intervention was not explicitly aimed at developing and sustaining such leadership.

\section{Data Culture}

A school culture that fosters data use and which is achievement oriented is considered important for implementing and sustaining DBDM in schools. In study 6 (chapter 7), the data culture of schools participating in the Focus intervention was studied.

Findings indicated that schools with initially high data-culture levels maintained the high standard and that schools with low levels of data culture showed an improvement. Whereas $59 \%$ of the schools were initially assigned to the lowest classes of data culture, the percentage dropped to 13 after the intervention.

Furthermore, school leaders are considered essential in implementing DBDM, among other things, because they can influence the data culture of their schools. Therefore, the relationship between leadership for DBDM and data culture was also investigated. It appeared that schools with more DBDM oriented school leaders were associated with higher levels of data culture.

\section{Collaboration}

To expand our knowledge on (changes in) collaboration patterns within a school during a DBDM intervention, in the seventh study (chapter 8), social network analysis was used to answer the research question: How do collaboration patterns within schools at the start of a $D B D M$ reform look like and how do they change during that reform? Strong collaboration was assumed to be a precondition for effective DBDM implementation, as teachers in such teams may have 1) access to the knowledge and skills for analyzing data, 2) the opportunity to discuss challenging performance goals, and 3) share effective teaching strategies to achieve those goals. Therefore, the intervention was offered as a team training and all intervention meetings included collaborative activities to strengthen collaboration patterns within the school. The density, reciprocity, and centralization of three DBDM-networks (discussing student achievement, goals, and instructional strategies) were analyzed using multivariate linear analysis.

Findings showed that prior to the intervention 'performance goals' were discussed to a lesser extent within school teams than 'student achievement' and 'instructional strategies'. The number of mutual relationships was highest for discussing student achievement and lowest for discussing goals. The student achievement network was mostly centralized, suggesting that a few influential teachers were central to the network.

After participating in the DBDM intervention for one year, no significant changes were found in teachers' collaboration patterns when it came to discussing goals and instructional strategies. However, although the number of relationships in the achievement network remained stable, the number of mutual relationships increased suggesting that more 
complex knowledge regarding student achievement was shared between staff members. Moreover, centralization of the achievement network decreased suggesting that the network had become less centralized.

\section{Comparing Characteristics for Schools with Different Intervention Effects}

In the first and second study (chapters 2 and 3), an overall positive intervention effect on student outcomes was found. However, we also found considerable variation between intervention effects across schools - while in some schools student achievement growth improved from prior compared to during the intervention, no improvement in student performance growth was found in other schools. These differences in intervention effects might be explained by educator and school organizational characteristics. In study 3 to 7 , we investigated these characteristics before, during, and after the DBDM intervention. In the final study (chapter 9), the relationship between the intervention effect on student achievement growth and the educator and school organizational factors was examined in order to identify what differentiates schools that benefited from the DBDM intervention from those that did not.

A comparison was made between ten schools with no intervention effects on students' mathematics achievement growth and ten schools with strong intervention effects on student mathematics achievement growth. Quantitative data gathered from the previous chapters were complemented with qualitative data gathered from project trainers and school leaders.

The school leader and trainer confirmed the influence of the educator and school organizational characteristics as included in our conceptual model on the implementation of DBDM. According to the school leaders and the trainers, (improved) knowledge and skills of school staff, a positive attitude towards DBDM, and good teaching quality skills had enhanced the DBDM-implementation process in the schools. Furthermore, a strong school leader, an achievement oriented data culture, and strong collaboration patterns were perceived as factors that had strengthened DBDM within the school.

Examination of the quantitative data, in particular, revealed that educators' attitude, teaching quality, and the schools' data culture were associated with strong effects on student achievement growth.

\section{Contributions}

The contribution of this dissertation to the existing research is twofold: on the one hand, it adds to the DBDM knowledge base as it shows how a DBDM intervention influences student outcomes. It reveals how educators' characteristics and school organizational factors can change during, as well as influence a DBDM implementation. On the other hand, this dissertation contributes to science in a methodological way. The intervention evaluated in this dissertation lasted two years, occurred in a real school context, and multiple measures collected at different levels (school, teacher, and student) were analyzed. Advanced statistical methods were required to deal with the data structure and to be able to accumulate all evidence to properly answer the research questions. In the next section, we first reflect on the contributions of this dissertation to DBDM research, followed by a reflection on its methodological contributions. 


\section{Contributions to DBDM Research}

The influence of a DBDM intervention on student outcomes. First, this dissertation contributes to the DBDM research base by providing evidence for the widespread claim that DBDM has a positive effect on student outcomes (Turner \& Coburn, 2012). High-quality standardized tests were used to uncover a main intervention effect approximately amounting to one month of extra schooling during the two intervention years. This effect of the DBDM intervention on student outcomes was uncovered in two separate cohorts of primary schools. The fact that we found an effect of the intervention on student outcomes in two different cohorts strengthens the consistency and generalizability of our findings (Makel \& Plucker, 2014; Schmidt, 2009). Replication studies are needed but are not frequently conducted, and when they are, the outcomes often differ (Hedges, 2016; Ioannidis, 2005). In the current study, a successful (conceptual) replication was conducted and the effects of mathematics on student outcomes were similar. Furthermore, this study revealed that not only student achievement growth for mathematics improved during the two intervention years but the same effect was also found for spelling achievement.

Moreover, the effect of DBDM on student outcomes was found in a large-scale study with approximately 4,000 students (FIII: spelling), 4,500 students (FIII: mathematics), and 7,500 students (FII). Based on the analysis of 645 intervention studies, Chueng and Slavin (2015) found a strong relationship between the scale of implementation of an intervention and the size of the intervention effects. The larger the number of students involved, the lower the effect sizes found. Hence, an effect of approximately one month of extra schooling during two intervention-years for 4.000-7.500 students found in this study by means of a standardized achievement test can be regarded as substantial.

Due to the inclusion of multiple intervention components, which is a typical feature of a comprehensive school reform (Desimone, 2002; Timperley, 2008), it is difficult to establish which particular intervention features led to these outcomes. We think it is the sum of its parts that has contributed to improved student outcomes. From a content point of view, the intervention was more comprehensive than other DBDM interventions, as it paid attention not only to the first step of DBDM (analyzing data) but to the whole DBDM cycle (including executing planned instructional strategies within the classroom). Second, involving entire school teams, instead of one or two important actors within schools, contributed to the implementation of DBDM throughout the whole school organization. Moreover, taking into account important features of effective professional development when developing the intervention, in combination with the explicit attention for the factors that were regarded as essential for DBDM-implementation in the literature, likely contributed to the intervention effects found.

Changing educator characteristics and school organizational characteristics. The second contribution made by this project to the DBDM research base was showing that the factors considered influential to DBDM-implementation are not stable but change in response to intervention, and, as such, improve DBDM-implementation in the school.

Desimone (2009) proposed a model in which she indicated how professional development can influence student outcomes. In her model, improved student outcomes as a result of an intervention can be achieved through improved teacher knowledge and skills, changes in teachers' attitudes and beliefs, and subsequently via changes in classroom instruction. In the model, context (i.e. teacher and student characteristics, school leadership, culture) is regarded as mediator and moderator for this process. This dissertation shows 
that, next to changes in teachers' knowledge and instruction skills (data literacy and teaching quality), a DBDM intervention can also influence contextual factors like school leadership, data culture, and collaboration patterns within a school.

In chapter 9, the initial situation for the schools' data culture, educators' attitudes, and teaching quality seemed to be crucial for the intervention effects. Schools with strong intervention effects on student achievement growth already had a strong achievement oriented data culture at the start of the intervention, their team members had a positive attitude towards DBDM, and teaching quality in those schools was high. In contrast, schools that achieved no intervention effects on student achievement growth initially scored lower on these three factors. During the two intervention years, the 'DBDM-factors' improved but student achievement growth was not affected by the intervention. Probably, for a DBDM intervention to be effective, schools first must meet specific preconditions for DBDMimplementation.

\section{Methodological Contributions}

Variety of instruments. In this dissertation, multiple factors were studied and a variety of measurements methods were used, varying from student achievement data on standardized tests to a teacher data literacy test, and a student perceptions questionnaire. Although research into DBDM has increased rapidly (e.g. Boudett, City, \& Murnane, 2005; Carlson, Borman, \& Robinson, 2011b; Love, Stiles, Mundry, \& DiRanna, 2008; Ritzema, 2015; Schildkamp, Poortman, \& Handelzalts, 2015; Slavin, Cheung, Holmes, Madden, \& Chamberlain, 2012), literature is lacking clear definitions of concepts such as data literacy, data culture, and collaboration for DBDM (Ritzema, 2015). As a result, instruments to measure these concepts were not available. Hence, for the purpose of this dissertation either new measurement instruments were developed (e.g. the data literacy test) or instruments from school-effectiveness research were used (e.g. instruments to measure school leadership, data culture, and teaching quality). Perceptions were used in the majority of the studies in this dissertation. There is some resistance against the use of perceptions, some researchers argue for example that student perceptions are not valid for measuring teaching quality in elementary education. Here we showed that, provided that appropriate scaling methods are used, perceptions are useful to measure teaching quality, as well as to measure school leadership, data culture or the collaboration within a school. Bayesian item response theory methods were used to address dependencies between respondents (students in chapter 5 or educators in chapters 6 and 7 ) in computing construct scores. The various scale-analysis results showed that reliable and consistent perception scores could be computed to address the perceptions of students or teachers. This shows that the perception responses represented consistent information about teaching quality, data culture, and school leadership.

Currently, in most studies using teacher or student perceptions, sum scores or mean scores of multiple Likert-scale items are calculated and subsequently aggregated at the school level to obtain a representative score of, for instance, school leadership (e.g. Heck \& Hallinger, 2009; ten Bruggencate, 2009). However, by using means or sum scores, between-person differences in response patterns leading to the same score are ignored. As a result, respondents with different response patterns obtain the same score, and valuable information about the response patterns is lost (Gorter, Fox, \& Twisk, 2015). In this study, multilevel IRT (leading to a continuous score) and multilevel LCA (leading to a categorical score) methods were used instead. In both types of analyses, participant response patterns were taken into account. Subsequently, these scores were used as dependent variables in 
the analysis of relationships between the measures perceptions and background variables.

Longitudinal analysis. In this dissertation, changes in multiple outcome variables at multiple levels (student, teacher, school) were analyzed over a two-year time period. We used three distinct approaches to explore the information from this multilevel longitudinal design. In the first two studies (chapter 2 and 3), growth was modeled by using linear mixed effect analysis. By means of adding random school effects, growth trajectories were examined per school. The method is based upon modeling explicitly the trajectories of change in performance using random effects. Therefore, this method requires a large number of repeated measures to estimate all model parameters. As a result, in chapter 2 and 3 it was not possible to include random grade effects, to distinguish student growth during the intervention between grades.

In the third and fourth study (chapter 4 and 5), growth was not explicitly parameterized; instead, changes in mean scores were analyzed using a pre-post multilevel IRT approach. For this approach, compared to the linear mixed effect analysis, fewer measurement points are needed. Therefore, this model was suitable for studies with only a pre- and a posttest measurement. The longitudinal multilevel structure of the data was accounted for by modeling the various cross-sectional and longitudinal correlations between measurements.

Finally, in the fifth and sixth study (chapter 6 and 7), transitions in school leadership classes and data culture classes were modeled by applying latent transition modelling. For each measurement point, (categorical) states were defined and subsequently changes from one state to another were modeled. Similar to the multivariate pre-post approach, this approach does not require a large number of data points and was therefore suitable for analyzing the variables measured three times during the intervention (e.g., educational leadership and data culture).

In sum, we showed how longitudinal data can be analyzed in various ways, depending on the number of data points and the nature of the outcome variable (continuous or categorical).

\section{Limitations and Directions for Future Research}

Below, a number of limitations regarding the studies presented in this dissertation will be discussed, as well as some directions for future research.

\section{Sample}

Schools participating in the Focus intervention were not sampled representatively from the Dutch population of primary schools, but were self-selected. Over a thousand primary schools were invited to participate in the intervention. As the need for improvement was highest in low-SES schools, these schools were initially invited. Some of the schools that were willing to participate belonged to a board that also governed medium-SES and high-SES schools. Some of these boards were only willing to participate with their schools, if all schools could participate.

Examination of the background characteristics of our sample schools revealed that the percentage of low-SES students in our schools was higher, on average, compared to the Dutch population. Moreover, schools in our sample were more often located in (sub)urban areas compared to the Dutch population. 
This discrepancy between the Dutch population of primary schools and our sample implies that we should take caution when generalizing our findings to the overall population of Dutch primary schools results. For more generalizable conclusions of the effectiveness of the DBDM intervention, a follow-up study using a representative sample is needed.

\section{Research Design}

This study was implemented in a real school context, hence, the inclusion of a control group appeared unfeasible. Schools simply were not willing to participate in a two-year intervention, provide student achievement data from their student monitoring system, and fill out surveys for the university, without being selected to be actively included in this intervention (or any other improvement program) to improve student performances.

In order to make causal inferences, in chapters 2, 3 and chapter 5, a multiple singlesubject design was used. Repeated measurements at the baseline (prior to the intervention) and during the treatment phase (during the intervention) were obtained, to measure accurately the change in performance over time, from the base line phase to the treatment phase. This single-subject design was applied to each school (or: teacher in chapter 5), meaning that each school served as its own control. A hierarchical modeling framework was applied to unite changes in performance across schools leading to an average estimate of change in performance. Furthermore, each school's estimated change in performance was partly based upon the performance change of the other schools, since they belong to the same population of schools. This principle of borrowing strength made it possible to simultaneously analyze the performance trajectories of schools, who were assigned to the same experimental design, and to improve the accuracy of each school's estimated change in performance.

Accordingly, the joint analysis of single-subject response data of the multiple schools made it possible to evaluate effects of the intervention across schools and for each school in specific. The intervention effects can be estimated by means of multilevel modelling (Shadish, Kyse, \& Rindskopf, 2013; Van Den Noortgate \& Onghena, 2003). Studying the effects of the intervention on teaching quality and student outcomes using a single-subject design (SSD) has the advantage that reliable and accurate school-specific or teacher-specific intervention effects can be determined. However, although SSD is becoming more prominent for testing intervention effects (Shadish et al., 2013; Shadish \& Sullivan, 2011; Sullivan, Shadish, \& Steiner, 2015), more research on these statistical methods is needed for making correct inferences in large-scale studies. In this dissertation, multilevel modeling methods were developed for categorical response data obtained via multiple single-subject designs. More research is needed to develop methods to address dependencies between subjects participating in comparable intervention programs, when multilevel modeling techniques do not provide to be useful to describe the subject's population distribution. For instance, when the grouping of subjects cannot be observed directly, or when the normal distribution does not correctly represent the distribution of subjects in the population. More research is also needed to study changes in performances in multiple topics, when for instance schools are measured repeatedly on different domains.

In the studies on collaboration patterns, school leadership, and data culture, the single-subject design analysis could not be applied, as multiple measurements were not available prior to the intervention to strengthen the statements concerning change in performance. Although these studies did broaden our understanding of the development of collaboration, school leadership, and data culture, we cannot claim that changes found 
during the intervention were caused by the intervention. Further research is needed to clarify changes in these factors as a result of DBDM interventions using longitudinal experimental designs including a control group.

\section{Selection of Research Questions}

Writing a joint dissertation provided the opportunity to study multiple aspects of the implementation and effects of a DBDM intervention. However, as pointed out in chapter 1 , not all aspects of DBDM could be studied.

In the previous section, we emphasized the importance of including all DBDM components in a DBDM intervention. In this study, we were not able to study the effects of the DBDM intervention on teachers' ability to set SMART and challenging goals (the second DBDM component) and the quality of determining strategies for goal-accomplishment (the third DBDM component). No data was collected on these two components, which could potentially be addressed in future studies. We expect that, in order to achieve improved student outcomes, the third component is especially important for the execution of DBDM in the classroom. When teachers are able to deliberately select the right instructional strategy to meet students' needs, improved student outcomes are likely.

Moreover, Figure 1 in chapter 1 shows that DBDM can take place at all levels of education: at the board (or a district), the school, and the classroom level. In this study, we mainly focused at the school level and to a lesser extent at the classroom (i.e. teacher) level. Although all schools in our sample served a board and this board was informed about the intervention by the trainers of the board's schools, the effects of the DBDM intervention at the board-level were not the central focus of this dissertation. We recommend to study 1) how the board level can impact DBDM implementation in their schools, and 2) how a DBDM intervention at the school level might affect how a board deals with DBDM.

Finally, in chapters 2 and 3, the average intervention effect was calculated for each school. To broaden our understanding of the effects of DBDM on student outcomes, it would be interesting to disentangle the school intervention effect into a grade effect. This was not possible in the analyses we used in chapter 2 and 3 due to the large amount of randomized effects that had to be estimated when modelling changes in student achievement from grade three to eight for each student. In future work, it would be interesting to find a way to model these grade effects.

\section{Implications for Practice}

Schools are faced with an increasing amount of student performance data, and teachers and school leaders can benefit from these data. However, data use is still not common practice in primary schools (Inspectie van het Onderwijs, 2014). In pre-service training relatively little attention is dedicated to equipping educators for the full utilization of such data (Bron, Geel, \& Visscher, 2013; Mandinach, Gummer, \& Muller, 2011; Mandinach \& Gummer, 2013a; Popham, 2011). Full utilization means that educators know how to use the systems as such (e.g., retrieving and interpreting the data) and, even more important, using these data for optimizing their instruction activities. Thus, DBDM should be incorporated much more in the curricula of pre-service institutions.

As many in-serve teachers also lack the competences for benefitting fully from DBDM (Inspectie van het Onderwijs, 2014), they also should be trained. In both, preservice and in-service training, the nature of the training activities is crucial. We argue that 
teachers' professional development activities should incorporate all components of DBDM (from analyzing data, to executing strategies within the classroom). Since success or failure of DBDM implementation is very dependent on the quality of the last component of the DBDM cycle, we recommend that, when developing a DBDM school reform, much attention is paid to executing DBDM in the classroom. For example, by including teacher classroom coaching, to provide teachers with feedback on how they can adapt their instruction in line with students' needs (as determined by means of student performance data) (Van der Scheer \& Visscher, 2014).

To conclude, professional development can remedy problems concerning a lack of DBDM knowledge and skills in current practice. When all teachers, both in-service as well as pre-service, apply DBDM as part of their common practice, education for all students can be enhanced. 


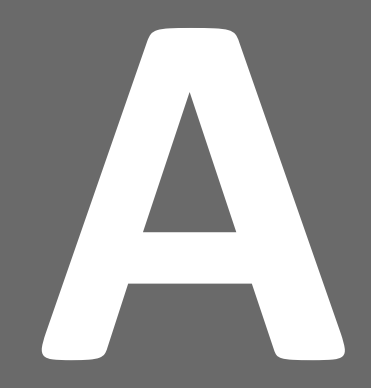

Appendices 


\section{Appendix A}

\section{A Detailed Explanation of the Equations for Each Model in Chapter 2}

Let $\tilde{g}=1$ refer to the first measurement occasion (mid-grade 3 ), $\tilde{g}=2$ refer to the average performance in end-grade 3 to grade 5 , and $\tilde{g}=3$ to the average performance in grade 6 to 8. Subsequently, the $\delta_{\tilde{g} i j}$ refers to the average performance of student $i$ in school $j$ in cluster $\tilde{g}$. Therefore, the change in performance of each student is modeled by three individual random effects.

The scores of each student are assumed to be independently and normally distributed given the population-average performance at each measurement occasion $t$, and the individual random effects, representing the individual deviation in performance at level mid-grade 3 , end-grade 3 to 5, and grade 6 to 8, from the population-average performance. Furthermore, the individual performances are assumed to be independent given the deviation of the school performance from the population average. Then, Model Oa can be stated as

$$
\begin{aligned}
& Y_{t g i j}=\mu_{t g}+\delta_{\tilde{g} i j}+e_{t g i j} \\
& \delta_{\tilde{g} i j}=\beta_{0 j}+u_{\tilde{g} i j} \\
& \beta_{0 j}=r_{0 j}
\end{aligned}
$$

The residual variance of the error component $e_{t g i j}$, and the variance of the random effect at level $2, r_{0 j}$, are assumed to be independently and normally distributed. The error terms $\left(u_{1 i j}, u_{2 i j}, u_{3 i j}\right)$ are assumed to be multivariate normally distributed such that the individual performances are allowed to correlate. This model was fitted using Imer using the following program call,

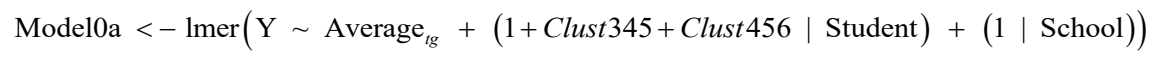

where Student and School are the two cluster variables, and Average represents the fixed factor variable of the population-average performance of each measurement occasion.

The model is extended to include a school-specific intervention effect using the variable Int $_{j}$, which is allowed to correlate with the school performance. That is, the error components $\left(r_{0 j}, r_{1 j}\right)$ are assumed to be multivariate normally distributed. The Model 1 is represented by

$$
\begin{aligned}
& Y_{t g i j}=\mu_{t g}+\delta_{\tilde{g} i j}+e_{t g i j} \\
& \delta_{\tilde{g} \tilde{j} j}=\beta_{0 j}+\beta_{1 j} I n t_{g i j}+u_{\tilde{g} i j} \\
& \beta_{0 j}=r_{0 j} \\
& \beta_{1 j}=\gamma_{10}+r_{1 j}
\end{aligned}
$$

and the call to Imer is given by

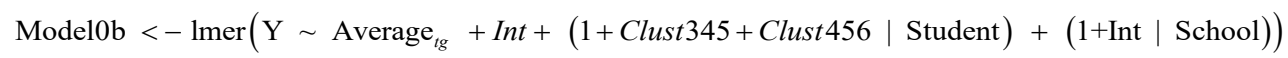


In model Ob the fixed effect of the intervention variable was not included. In Model 2, student characteristics with fixed effects are added to the mean term. A factor variable SES with three levels (low, medium, high), Gender (Female is coded as one), and a variable Age (which is centered around the expected age at each measurement occasion). This Model 2 is given by,

$$
\begin{aligned}
& Y_{t g i j}=\mu_{t g}+\delta_{\tilde{g} i j}+\beta_{2} \text { SES }_{i j}+\beta_{3} \text { Gender }_{i j}+\beta_{4} \text { Age }_{t g i j}+e_{t g i j} \\
& \delta_{\tilde{g} i j}=\beta_{0 j}+\beta_{1 j} \text { Int }_{g i j}+u_{\tilde{g} i j} \\
& \beta_{0 j}=r_{0 j} \\
& \beta_{1 j}=\gamma_{10}+r_{1 j}
\end{aligned}
$$

and the call to Imer is given by

$$
\begin{aligned}
& \text { Model } 2<-\operatorname{lmer}\left(\mathrm{Y} \sim \text { Average }_{t g}+\text { Int }+ \text { SES }+ \text { Gender }+ \text { Age }+\right. \\
& \left.(1+\text { Clust } 345+\text { Clust } 456 \mid \text { Student })_{+}+(1+\text { Int } \mid \text { School })\right)
\end{aligned}
$$

In Model 3, school-level explanatory variables are added. The variables school size (small, medium large), urbanization (rural, suburban, urban), and school-level SES (low, medium, high) were added. This leads to

$$
\begin{aligned}
& Y_{t g i j}=\mu_{t g}+\delta_{\tilde{g} i j}+\beta_{2} \text { SES }_{i j}+\beta_{3} \text { Gender }_{i j}+\beta_{4} \text { Age }_{t g i j}+e_{t g i j} \\
& \delta_{\tilde{g} i j}=\beta_{0 j}+\beta_{1 j} \text { Int }_{g i j}+u_{\tilde{g} i j} \\
& \beta_{0 j}=\gamma_{01} \text { Size }_{j}+\gamma_{02} \text { Urbanization }_{i j}+\gamma_{03} \text { SES }_{j}+r_{0 j} \\
& \beta_{1 j}=\gamma_{10}+r_{1 j}
\end{aligned}
$$

and the call to Imer is given by

$$
\begin{aligned}
& \text { Model } 3<-\operatorname{lmer}\left(\mathrm{Y} \sim \text { Average }_{t g}+\text { Int }+ \text { SES }+ \text { Gender }+\right. \text { Age } \\
& + \text { Size }+ \text { Urbanization }+ \text { School-SES }+ \\
& (1+\text { Clust } 345+\text { Clust } 456 \mid \text { Student })+(1+\text { Int } \mid \text { School })) .
\end{aligned}
$$

In Model 4, the significant predictor variables of Model 3 are included, and the intervention trajectory (school variable Trajectory) was added,

$$
\begin{aligned}
& Y_{t g i j}=\mu_{t g}+\delta_{\tilde{g} i j}+\beta_{2} \text { SES }_{i j}+\beta_{3} \text { Gender }_{i j}+\beta_{4} \text { Age }_{t g i j}+e_{t g i j} \\
& \delta_{\tilde{g} i j}=\beta_{0 j}+\beta_{1 j} \text { Int }_{g i j}+u_{\tilde{g} i j} \\
& \beta_{0 j}=\gamma_{01} \text { SES }_{j}+\gamma_{02} \text { Traject }_{j}+r_{0 j} \\
& \beta_{1 j}=\gamma_{10}+r_{1 j}
\end{aligned}
$$

with the corresponding call to Imer, 


$$
\begin{aligned}
& \text { Model } 4<-\operatorname{lmer}\left(\mathrm{Y} \sim \text { Average }_{t g}+\text { Int }+ \text { SES }+ \text { Gender }+\right. \text { Age } \\
& + \text { School-SES }+ \text { Traject }+ \\
& (1+\text { Clust } 345+\text { Clust } 456 \mid \text { Student })+(1+\text { Int } \mid \text { School })) .
\end{aligned}
$$

Finally, in Model 5, interaction effects were added for the of school and student SES with the intervention variable. Model 5 is given by

$$
\begin{aligned}
& Y_{t g i j}=\mu_{t g}+\delta_{\tilde{g} i j}+\beta_{2} S E S_{i j}+\beta_{3} \text { Gender }_{i j}+\beta_{4} \text { Age }_{t g i j}+e_{t g i j} \\
& \delta_{\tilde{g} i j}=\beta_{0 j}+\beta_{1 j} \text { Int }_{g i j}+\beta_{5} \text { Int }_{g i j} S E S_{i j}+u_{\tilde{g} i j} \\
& \beta_{0 j}=\gamma_{01} S E S_{j}+r_{0 j} \\
& \beta_{1 j}=\gamma_{10}+\gamma_{20} S E S_{j}+r_{1 j}
\end{aligned}
$$

and the call to Imer is given by

$$
\begin{aligned}
& \text { Model5 }<-\operatorname{lmer}\left(\mathrm{Y} \sim \text { Average }_{t g}+\text { Int }+ \text { SES }+ \text { Gender }+\right. \text { Age } \\
& + \text { School-SES }+(\text { School-SES } * \text { Int })+(\text { SES } * \text { Int })+ \\
& (1+\text { Clust } 345+\text { Clust } 456 \mid \text { Student })+(1+\text { Int } \mid \text { School })) .
\end{aligned}
$$




\section{Appendix B}

\section{Data Literacy test}

\section{General Data Literacy - Possibilities (11 items)}

\begin{tabular}{|c|c|c|}
\hline & With our SMS, we can ... & (Answer) \\
\hline$\overline{\text { GP.01 }}$ & $\begin{array}{l}\text {... compare the scores of our students with national aaverage } \\
\text { scores on the SMS math tests }\end{array}$ & (true \\
\hline GP.02 & $\begin{array}{l}\text {... compare the scores of our students with those of schools with } \\
\text { similar student population composition }\end{array}$ & (false) \\
\hline GP.03 & ... represent the pedagogical quality of each teacher & (false) \\
\hline GP.04 & $\begin{array}{l}\text {... compare the scores of the current grade } 5 \text { with their own } \\
\text { achievement in previous school years }\end{array}$ & (true) \\
\hline GP.05 & $\begin{array}{l}\text {... compare the scores of our students with those of students in } \\
\text { the same grade, in other countries }\end{array}$ & (false) \\
\hline GP.06 & $\begin{array}{l}\text {... determine the achievement growth of grade } 6 \text {, from the test } \\
\text { End grade } 5 \text { and End grade } 6\end{array}$ & (true) \\
\hline GP.07 & $\ldots$ not show the scores of all grades at the same time & (false) \\
\hline GP.08 & $\begin{array}{l}\text {... check whether students' scores are in line with their } \\
\text { intelligence (IQ) }\end{array}$ & (false) \\
\hline GP.09 & $\begin{array}{l}\text {... see how mathematics scores would improve if we would } \\
\text { dedicate more time to math lessons }\end{array}$ & (false) \\
\hline GP.10 & $\begin{array}{l}\text {... compare the achievement growth for grade } 4 \text { math with } \\
\text { achievement growth for grade } 4 \text { reading }\end{array}$ & (true) \\
\hline GP.11 & $\begin{array}{l}\text {... not compare the math achievement of the current grade } 5 \text {, } \\
\text { with math achievement of previous grade } 5\end{array}$ & (false) \\
\hline
\end{tabular}

\section{General Data Literacy - Interpreting Benchmarks (2 items)}

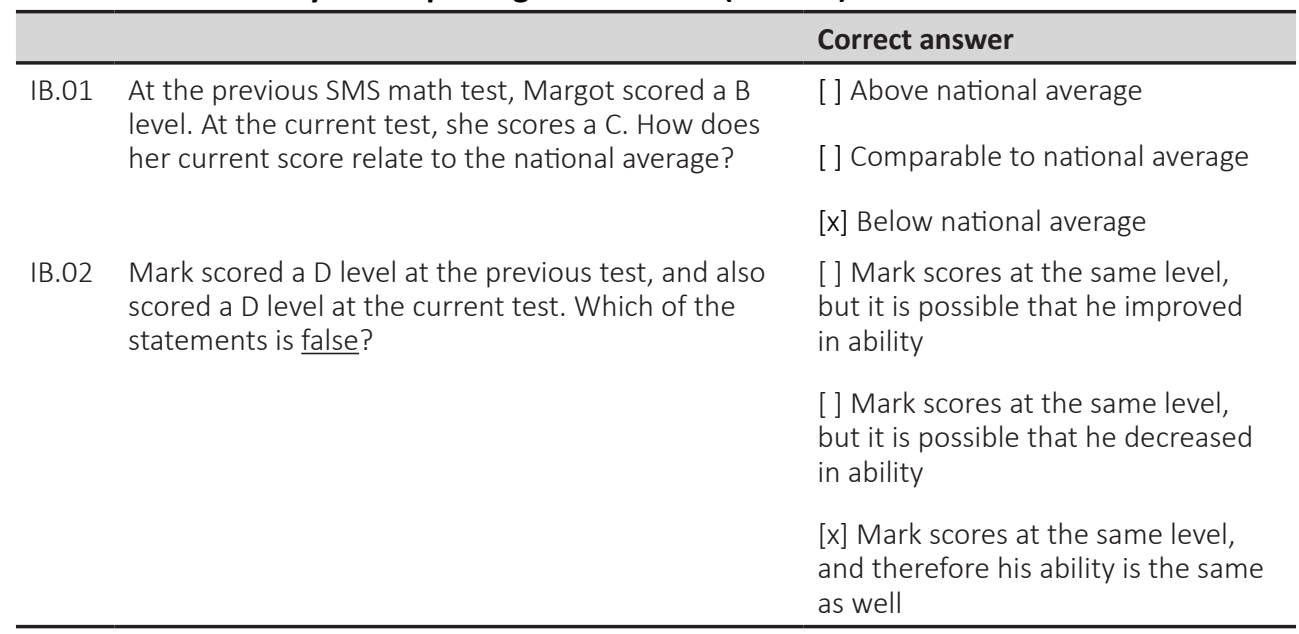


General Data Literacy - System Specific Answer (2 items)

\begin{tabular}{lllll}
\hline & With our SMS, we can ... & C & E & P \\
\hline GS.01 & $\begin{array}{l}\text {... determine ability growth for individual students, be- } \\
\text { tween the test End grade 4 and End grade 5 }\end{array}$ & true & false & true \\
GS.02 & $\begin{array}{l}\text { k... see how many mistakes students made for specific } \\
\text { categories of mathematics and spelling items }\end{array}$ & true & true & false \\
\hline
\end{tabular}

\section{Analyses in System C (15 items)}

\begin{tabular}{|c|c|c|}
\hline & & Correct answer \\
\hline KC.01 & $\begin{array}{l}\text { Use Figure C1. What can you say about the scores } \\
\text { of 'groep 6a' (black box), compared to the national } \\
\text { average? }\end{array}$ & $\begin{array}{l}\text { [x] Above national average } \\
\text { [] Comparable to national average } \\
\text { [] Below national average }\end{array}$ \\
\hline KC.02 & $\begin{array}{l}\text { Use Figure C1. Statement: The distribution of } \\
\text { scores of 'groep } 4 b^{\prime} \text { (blue box) is comparable to the } \\
\text { national average distribution of scores }\end{array}$ & $\begin{array}{l}\text { [] True } \\
\text { [] False }\end{array}$ \\
\hline KC.03 & $\begin{array}{l}\text { Use Figure C2. What can we say about grade } 5 \text { in } \\
\text { school year 2008-2009? }\end{array}$ & $\begin{array}{l}\text { [ ] Students in grade } 5 \text { made the } M 6 \\
\text { test instead of the E5 test } \\
\text { [X] At E5 test occasion, students in } \\
\text { grade } 5 \text { scored an average ability } \\
\text { score which was higher than the } \\
\text { average score at M6 } \\
\text { [] Based on the score at E5, a score } \\
\text { of } 85 \text { on } M 6 \text { is expected }\end{array}$ \\
\hline KC.04 & $\begin{array}{l}\text { Use Figure C2. What can you say about the score of } \\
\text { grade 6, at the end of school year 2007-2008? }\end{array}$ & $\begin{array}{l}\text { [x] Above national average } \\
\text { [] Comparable to national average } \\
\text { [] Below national average }\end{array}$ \\
\hline KC.05 & $\begin{array}{l}\text { Use Figure C2. Which of the following statements is/ } \\
\text { are true? } \\
\text { 1. The students who were in grade } 4 \text { in school year } \\
2006-2007 \text { scored the highest of all grades } 4 \text { in the } \\
\text { periode } 2005-2009 \\
\text { 2. The green line shows the development of } \\
\text { students who were in grade } 4 \text { in school year 2008- } \\
2009\end{array}$ & $\begin{array}{l}\text { [x] Statement } 1 \text { is true, statement } 2 \\
\text { is false } \\
\text { [] Statement } 1 \text { is false, statement } 2 \\
\text { is true } \\
\text { [] Both statement } 1 \text { and } 2 \text { are true } \\
\text { [] Both statement } 1 \text { and } 2 \text { are false }\end{array}$ \\
\hline KC.06 & $\begin{array}{l}\text { Use Figure C3. What can you say about the scores } \\
\text { of 'groep } 5 a^{\prime} \text { at the end of school year 2007-2008 } \\
\text { compared to the national average? }\end{array}$ & $\begin{array}{l}\text { [] Above national average } \\
\text { [] Comparable to national average } \\
\text { [] Below national average }\end{array}$ \\
\hline KC.07 & $\begin{array}{l}\text { Use Figure C3. What can you say about the } \\
\text { achievement of 'groep } 7 b^{\prime} \text { at the end of school year } \\
2007-2008 \text {, compared to their own achievement at } \\
\text { the end of school year 2006-2007? }\end{array}$ & $\begin{array}{l}{[x] \text { Their achievement is below }} \\
\text { expectations } \\
\text { [] Their achievement is according to } \\
\text { what you would expect } \\
\text { [] Their achievement is above } \\
\text { expectations }\end{array}$ \\
\hline
\end{tabular}


KC.08 Use Figure C4. What can you say about the development of achievement for mathematics (RekWisk 2010) and Vocabulary (Woordenschattoets 2010) of grade 4, from Mid 2008-2009 to Mid 20092010?

KC.09 Use Figure C5. Statement: In Figure 5, you can see which students had the highest score on the test.

KC.10 What is absolutely true about the three bars at the left in Figure C5?

KC.11 What is absolutely true about the outer right bar in Figure $\mathrm{C} 5$ ?

KC.12 Statement: The student represented by the outer right bar in Figure 5, cannot have scored an A at this test.

KC.13 Statement: For the category 'Numbers and number relations' (GET), Daisy Hart scores better than expected, based on her overall ability score

KC.14 Statement:

Iris van Ham made no mistakes in the category

'Addition and Subtraction' (O\&A)

KC.15 Statement:

The signal 'opvallend' or 'zeer opvallend' is based on the levels $A$ to $E$

KC.16 Statement: Almost all children have problems with regard to category 'Addition and Subtraction' (O\&A)
[] Both Mathematics achievement and Vocabulary achievement develop from below to above national average.

[x] Mathematics achievement develops from below, to above national average. Vocabulary achievement develops from below to far below national average.

[] Vocabulary achievement develops from below, to above national average. Mathematics achievement develops from below to far below national average.

False

[] These students score at level A $[x]$ These students show an ability growth far above national average growth

[] We cannot determine the level of these students, but they do perform at the same level

[] This student scores at level $E$ $[x]$ This student's ability decreased [] This student didn't take the test

False

True

False

False

True

Similar questions were available for system $E$ and $P$ (in the final version of this manuscript, $a$ translation of these items will be provided). 
Data Literacy Test - Figures

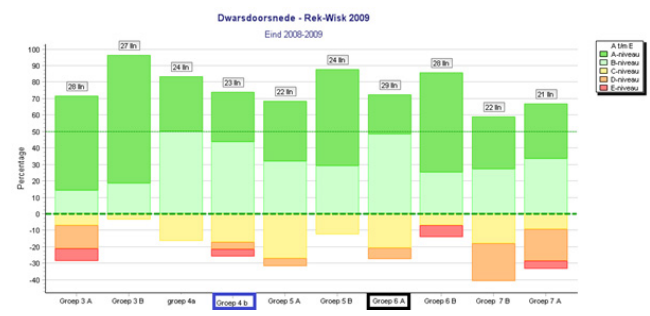

Figure C1

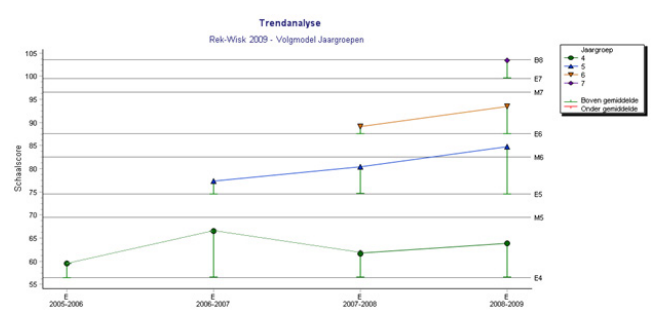

Figure C2

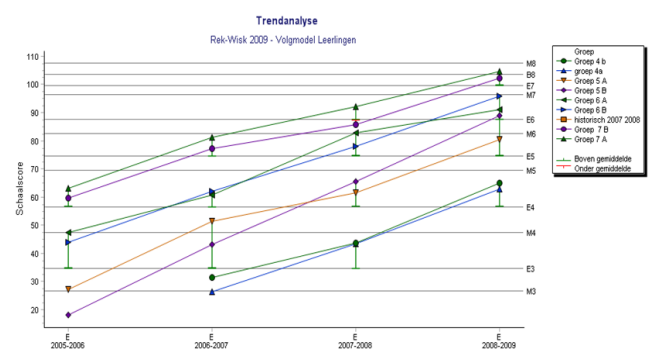

Figure C3

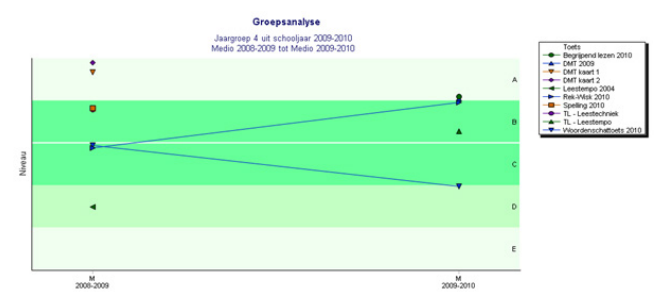

Figure C4 


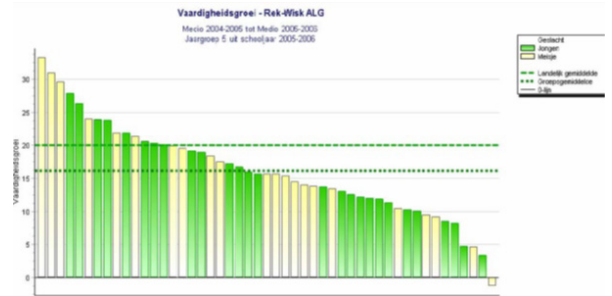

\section{Figure C5}

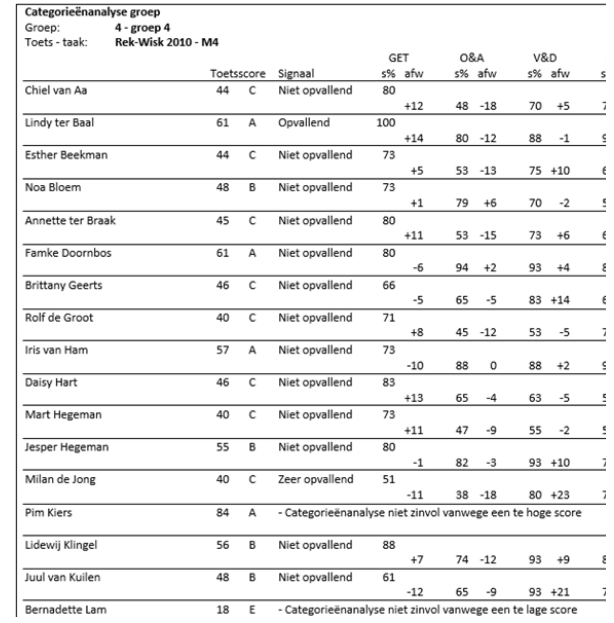

Figure C6

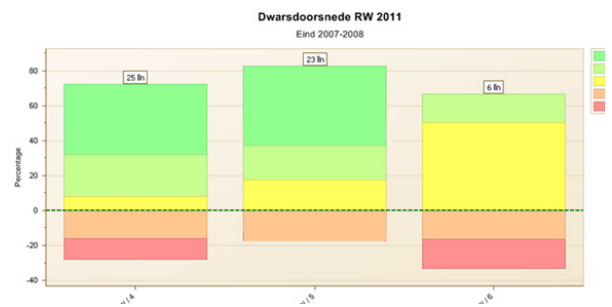

\section{Figure E1}

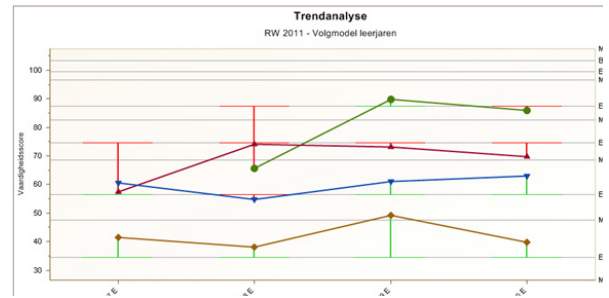

Figure E2 


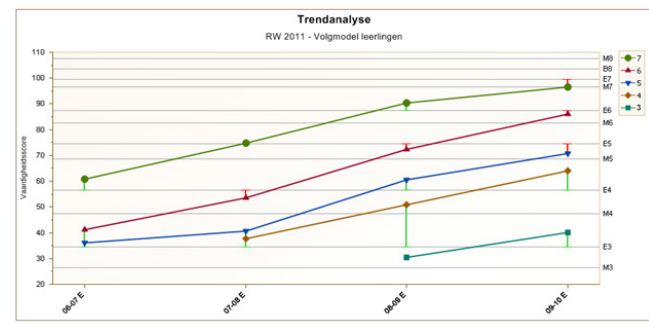

Figure E3

\begin{tabular}{|c|c|c|c|c|c|c|c|c|}
\hline $\begin{array}{l}\text { Aantal opgaven: } \\
\text { Leerling }\end{array}$ & $\begin{array}{l}\text { Totaal } \\
56\end{array}$ & $\begin{array}{l}\text { Cat1 } \\
9\end{array}$ & $\begin{array}{l}\text { Cat2 } \\
10\end{array}$ & $\begin{array}{l}\text { Cat3 } \\
6\end{array}$ & $\begin{array}{l}\text { Cat4 } \\
10\end{array}$ & $\begin{array}{l}\text { Cat10 } \\
3\end{array}$ & $\begin{array}{l}\text { Cat19 } \\
4\end{array}$ & $\begin{array}{l}\text { Cat20 } \\
14\end{array}$ \\
\hline Devi & 37 & 7 & 6 & 4 & 10 & 2 & 2 & 6 \\
\hline Maud & 44 & 8 & 9 & 3 & 9 & 3 & 2 & 8 \\
\hline Thomas & 48 & 9 & 7 & 4 & 9 & 3 & 3 & 13 \\
\hline Beate & 51 & 9 & 9 & 5 & 10 & 2 & 2 & 14 \\
\hline Jarvo & 52 & 9 & 10 & 5 & 10 & 3 & 3 & 12 \\
\hline Rachel & 49 & 8 & 9 & 4 & 9 & 3 & 4 & 12 \\
\hline Wieke & 43 & 9 & 8 & 3 & 10 & 1 & 3 & 9 \\
\hline Collin & 38 & 7 & 8 & 4 & 5 & 3 & 2 & 9 \\
\hline Ahmed & 46 & 8 & 10 & 3 & 9 & 2 & 3 & 11 \\
\hline Fleur & 37 & 8 & 8 & 4 & 6 & 1 & 2 & 8 \\
\hline Linda & 38 & 7 & 8 & 5 & 7 & 1 & 2 & 8 \\
\hline Frank & 21 & 5 & 3 & 3 & 6 & 2 & 0 & 2 \\
\hline Eli & 51 & 9 & 9 & 5 & 10 & 2 & 2 & 13 \\
\hline so & 53 & 9 & 10 & 3 & 10 & 3 & 4 & 14 \\
\hline Puk & 51 & 9 & 10 & 5 & 9 & 2 & 3 & 13 \\
\hline Guus & 45 & 7 & 8 & 4 & 10 & 1 & 2 & 13 \\
\hline Silke & 33 & 5 & 6 & 4 & 8 & 1 & 2 & 7 \\
\hline Najib & 35 & 6 & 7 & 4 & 8 & 2 & 1 & 7 \\
\hline Mounir & 42 & 7 & 8 & 3 & 10 & 1 & 2 & 11 \\
\hline Sybel & 40 & 7 & 6 & 3 & 8 & 3 & 3 & 10 \\
\hline Gemiddeld & 42,7 & 7 & 8 & 4 & 9 & 2 & 2 & 10 \\
\hline
\end{tabular}

Cat1: RW01-G-GR/ Getallen en getalrelaties
Catz: RW02-HR-OA / HR. optellen en afrekken

Car3: RW03.HR.VD / HR vermenigv. en delen

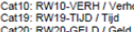

\section{Figure E4}

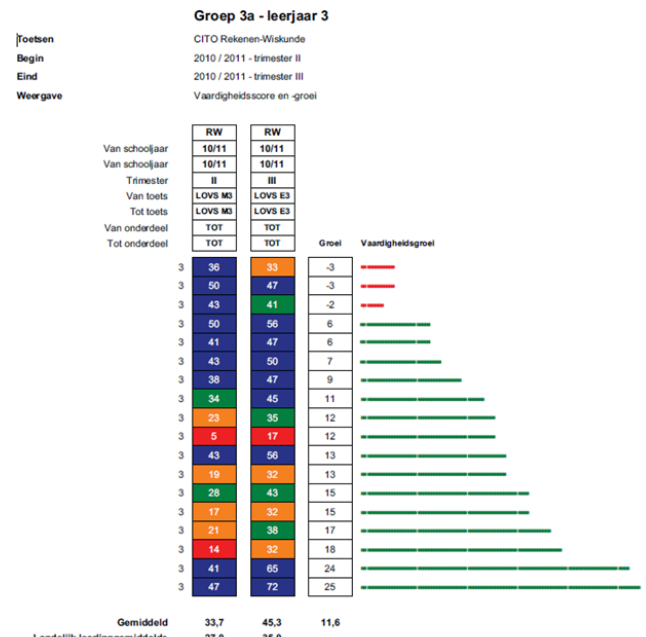

Figure P1 
Periode 2008/2009

Ontwikkeling niveaupercentages A, B, C en D/E

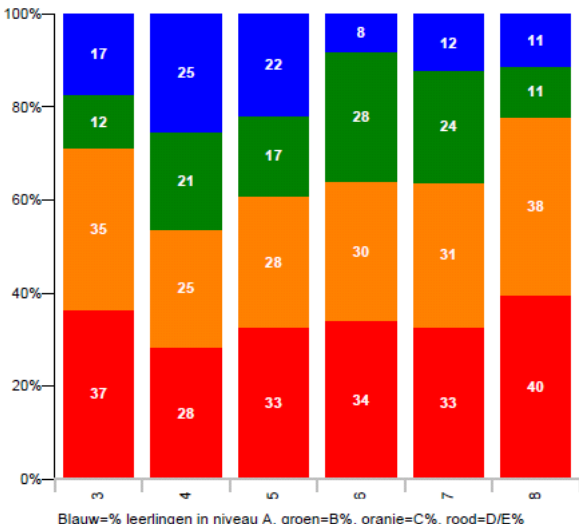

Figure P2

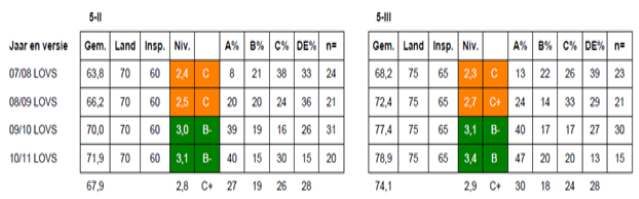

Figure P3

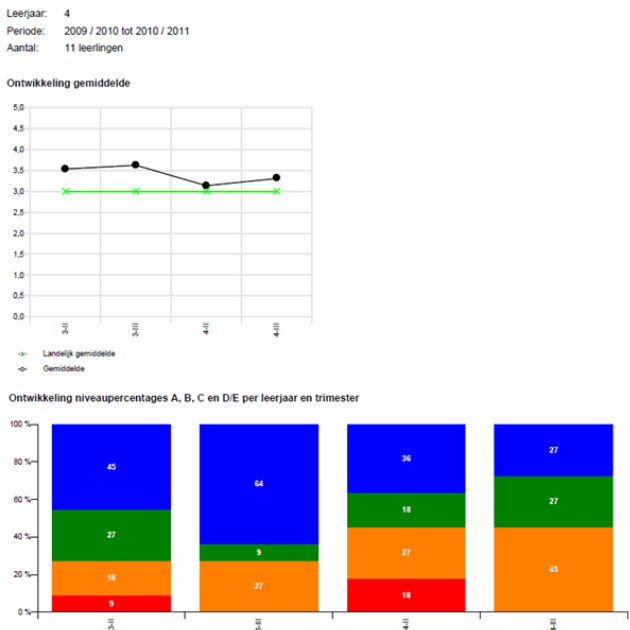

Figure P4 


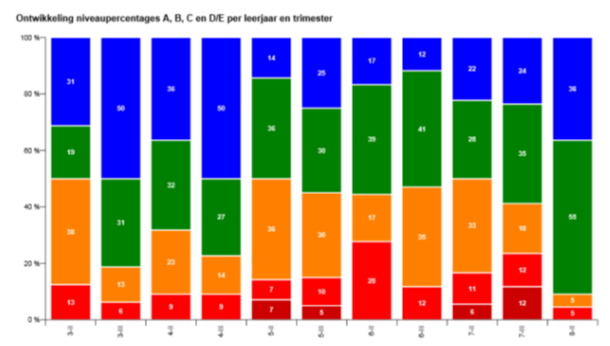

Figure P5

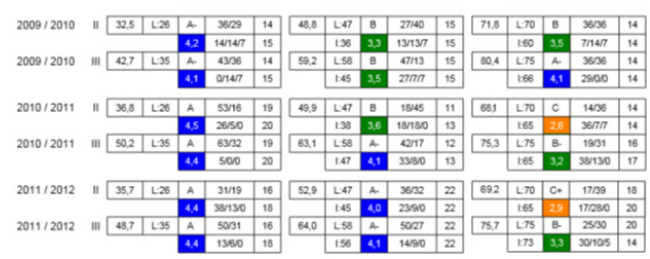

\section{Figure P6}

6 (leerjaar 6) 4. Rekenen en wiskunde: CITO Rekenen-Wiskunde LOVS-Totaal Hootvese in $2000 / 2010-2011 / 2012$

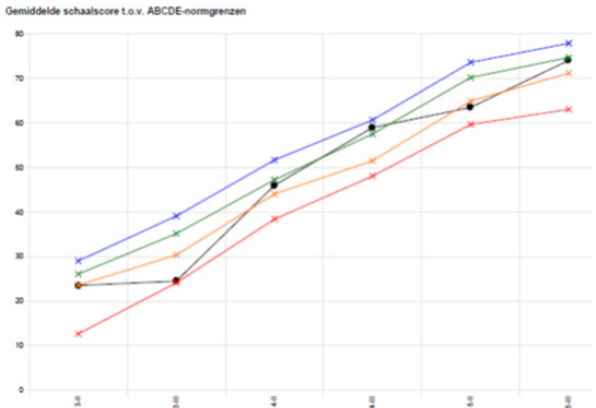

Figure P7 


\section{Appendix C}

\section{Appendix to chapter 5: "The Development of Teaching Quality During a DBDM intervention: A Pre-Post Multilevel IRT Approach"}

In this appendix, the item parameters of all items are examined first. Next, the findings of the analyses for the scales 'creating a safe climate', 'classroom management', 'providing clear instruction', and 'challenging students' are presented. For each scale, the results of the empty model are presented, followed by the results of the final model. Explanatory variables were included in a stepwise manner. Only the explanatory variables that had a significant effect on the pretest and/or posttest factor scores were included in the final model. A brief summary of the results is given in the results section in the article itself.

\section{Discrimination and Difficulty of the Items}

Table A.1 shows an overview of the items in the student questionnaire with the accompanying discrimination parameters per item at each measurement point. The accompanying thresholds are presented in Table A.2. In general, the thresholds showed that the teachers were most likely to acquire positive student ratings about their safe climate, suggesting that according to the students most teachers fostered a safe climate at both the start as well as at the end of the intervention. Similarly, at the pretest students were positive about their teachers challenging them to do their best. In contrast, it was less common for teachers to achieve positive students' ratings on the goal-orientation scale, suggesting that on average students did not think that their teachers acted in a goal-oriented way. To contextualize these findings, the scales are described.

The scale 'creating a safe climate' included six items. The discrimination parameters ranged from .54 to 1.07, which implied sufficient discriminating power to rank the students and teachers on a common scale. For all items, the estimated response category boundaries were well spread such that perceptions of students at the low and high ends of the scale could be accurately measured. Based on the thresholds, it appeared that students more often answered question 1.3 positively ('my teacher wants me to do well at school') compared to question 1.5 ('my teachers seems to know if something is bothering me').

The scale 'classroom management' consisted of seven items. The discrimination parameters ranged from .31 to .86, which implies low to moderate discrimination power. Item 2.3 ('We have clear rules in the classroom') had the lowest discrimination power. A possible explanation might be that based on the thresholds, the probability that teachers receive a positive response from students on this item is large, which in turn might have resulted in lower discrimination power. Conversely, item 2.6 ('everybody pays attention when my teacher explains something') was least likely to be answered positively by the students.

The 'providing clear instruction' scale included 10 items. The discrimination parameters ranged from .40 to 1.04, revealing low to sufficient discriminating power to rank the students and teachers on a common scale. In particular, item 3.3 ('When my teacher explains something I get it right away'), and item 3.4 ('My teacher wants me to explain how I got to my answer') had little discrimination power. These two questions might be hard to answer for students. The thresholds reveal, that especially for item 3.3 students rarely answered 'yes, always'. As item 3.6, 3.7, and 3.9 had high discrimination power, none of these items were excluded. 
Finally, the 'challenging students' scale included 5 items. The discrimination parameters ranged from little (.37) to high (1.23) discrimination power. Question 4.5 ('My teacher is only satisfied when we do the best we can') had little discrimination power. The thresholds reveal that for the 'challenging students' items it was relatively easy to achieve positive students ratings, compared to for example 'goal orientation'.

Table A.1 Translation of the student questionnaire and discrimination per item

\begin{tabular}{|c|c|c|c|c|c|}
\hline & & \multicolumn{2}{|l|}{ Pretest } & \multicolumn{2}{|c|}{ Posttest } \\
\hline & & Discrimination & (SD) & Discrimination & $(\mathrm{SD})$ \\
\hline & Creating a safe climate & & & & \\
\hline $1.1^{*}$ & $\begin{array}{l}\text { My teacher is nice to me when I ask } \\
\text { questions }\end{array}$ & .98 & $(.03)$ & .98 & $(.03)$ \\
\hline $1.2^{*}$ & $\begin{array}{l}\text { I like the way my teacher treats me } \\
\text { when I need help }\end{array}$ & 1.07 & $(.02)$ & 1.07 & $(.02)$ \\
\hline 1.3 & $\begin{array}{l}\text { My teacher wants me to do well at } \\
\text { school }\end{array}$ & .61 & $(.02)$ & .61 & $(.02)$ \\
\hline $1.4^{*}$ & $\begin{array}{l}\text { When I am sad or angry my teacher } \\
\text { helps me so I will feel better }\end{array}$ & .97 & $(.02)$ & .97 & $(.02)$ \\
\hline 1.5 & $\begin{array}{l}\text { My teacher seems to know if } \\
\text { something is bothering me }\end{array}$ & .68 & $(.02)$ & .69 & $(.02)$ \\
\hline \multirow[t]{2}{*}{1.6} & I like this class & .54 & $(.01)$ & .54 & $(.01)$ \\
\hline & $\underline{\text { Classroom management }}$ & & & & \\
\hline 2.1 & We start the lessons on time & .59 & $(.02)$ & .61 & $(.02)$ \\
\hline 2.2 & $\begin{array}{l}\text { When we are working individually, it is } \\
\text { quiet in the classroom }\end{array}$ & .72 & $(.02)$ & .71 & $(.02)$ \\
\hline 2.3 & We have clear rules in the classroom & .50 & $(.02)$ & .52 & $(.02)$ \\
\hline 2.4 & $\begin{array}{l}\text { I know when I can ask my teacher } \\
\text { questions during work time }\end{array}$ & .31 & $(.01)$ & .32 & $(.01)$ \\
\hline $2.5^{*}$ & Our classroom is neat and tidy & .72 & $(.02)$ & .72 & $(.02)$ \\
\hline $2.6^{*}$ & $\begin{array}{l}\text { Everybody pays attention when my } \\
\text { teacher explains something }\end{array}$ & .86 & $(.02)$ & .86 & $(.02)$ \\
\hline \multirow[t]{2}{*}{$2.7^{*}$} & Everybody in our class works hard & .75 & $(.02)$ & .75 & $(.02)$ \\
\hline & Providing clear instruction & & & & \\
\hline 3.1 & $\begin{array}{l}\text { My teacher explains difficult things } \\
\text { clearly }\end{array}$ & .71 & $(.02)$ & .71 & $(.02)$ \\
\hline 3.2 & $\begin{array}{l}\text { If I don't understand something my } \\
\text { teacher explains it another way }\end{array}$ & .69 & $(.01)$ & .70 & $(.01)$ \\
\hline 3.3 & $\begin{array}{l}\text { When my teacher explains something I } \\
\text { get it right away }\end{array}$ & .44 & $(.01)$ & .43 & $(.01)$ \\
\hline 3.4 & $\begin{array}{l}\text { My teacher wants me to explain how } \\
\text { I got to my answer }\end{array}$ & .40 & $(.01)$ & .42 & $(.01)$ \\
\hline 3.5 & $\begin{array}{l}\text { My teacher knows how he/she can } \\
\text { best explain something to me }\end{array}$ & .74 & $(.02)$ & .75 & $(.02)$ \\
\hline
\end{tabular}




\begin{tabular}{|c|c|c|c|c|c|}
\hline & & \multicolumn{2}{|l|}{ Pretest } & \multicolumn{2}{|c|}{ Posttest } \\
\hline & & Discrimination & $(\mathrm{SD})$ & Discrimination & $(\mathrm{SD})$ \\
\hline $3.6^{*}$ & $\begin{array}{l}\text { My teacher knows when I understand } \\
\text { something and when I do not }\end{array}$ & .95 & $(.02)$ & .95 & $(.02)$ \\
\hline $3.7^{*}$ & $\begin{array}{l}\text { My teacher helps me if I do not } \\
\text { understand something }\end{array}$ & 1.04 & $(.02)$ & 1.04 & $(.02)$ \\
\hline 3.8 & $\begin{array}{l}\text { My teacher asks questions to be sure I } \\
\text { understand }\end{array}$ & .64 & $(.01)$ & .65 & $(.01)$ \\
\hline $3.9 *$ & $\begin{array}{l}\text { My teacher explains things just as long } \\
\text { until I get it }\end{array}$ & .81 & $(.01)$ & .81 & $(.01)$ \\
\hline \multirow[t]{2}{*}{3.10} & $\begin{array}{l}\text { If my answer to a question is incorrect } \\
\text { my teacher explains why it is incorrect. }\end{array}$ & .68 & $(.01)$ & .68 & $(.01)$ \\
\hline & $\underline{\text { Challenging students }}$ & & & & \\
\hline $4.1^{*}$ & My teacher wants me to do my best & .85 & $(.02)$ & .85 & $(.02)$ \\
\hline $4.2^{*}$ & $\begin{array}{l}\text { My teacher thinks I can do good work } \\
\text { if I try my hardest }\end{array}$ & .86 & $(.02)$ & .86 & $(.02)$ \\
\hline 4.3 & $\begin{array}{l}\text { My teacher says that we need to } \\
\text { think carefully about how to do the } \\
\text { assignments well }\end{array}$ & .69 & $(.02)$ & .69 & $(.02)$ \\
\hline $4.4^{*}$ & $\begin{array}{l}\text { My teacher thinks I can learn } \\
\text { everything if I do my best }\end{array}$ & 1.23 & $(.03)$ & 1.23 & $(.03)$ \\
\hline \multirow[t]{2}{*}{4.5} & $\begin{array}{l}\text { My teacher is only satisfied when we } \\
\text { do the best we can }\end{array}$ & .37 & $(.01)$ & .38 & $(.01)$ \\
\hline & Goal-oriented teaching & & & & \\
\hline 5.1 & $\begin{array}{l}\text { My teacher tells us at the start of the } \\
\text { lesson what we are learning }\end{array}$ & .68 & $(.02)$ & .69 & $(.02)$ \\
\hline $5.2^{*}$ & $\begin{array}{l}\text { My teacher tells us at the start of the } \\
\text { lesson why we are learning }\end{array}$ & .86 & $(.02)$ & .86 & $(.02)$ \\
\hline 5.3 & $\begin{array}{l}\text { When my teacher marks my work, he/ } \\
\text { she writes comments on my papers to } \\
\text { help me understand }\end{array}$ & .58 & $(.01)$ & .58 & $(.01)$ \\
\hline $5.4^{*}$ & $\begin{array}{l}\text { My teacher asks us what we have } \\
\text { learned at the end of the lesson }\end{array}$ & 1.04 & $(.02)$ & 1.04 & $(.02)$ \\
\hline $5.5^{*}$ & $\begin{array}{l}\text { My teacher reminds us of what } \\
\text { we have covered previously at the } \\
\text { beginning of the lesson }\end{array}$ & .83 & $(.01)$ & .83 & $(.01)$ \\
\hline 5.6 & $\begin{array}{l}\text { My teacher wants me to think } \\
\text { carefully whether my answer is correct }\end{array}$ & .51 & $(.01)$ & .51 & $(.01)$ \\
\hline
\end{tabular}


Table A.2. Estimated category-bound parameters per item

\begin{tabular}{|c|c|c|c|c|c|c|c|c|}
\hline & \multicolumn{4}{|c|}{ Pretest } & \multicolumn{4}{|c|}{ Posttest } \\
\hline & 1 & 2 & 3 & 4 & 1 & 2 & 3 & 4 \\
\hline \multicolumn{9}{|c|}{ Creating a safe climate } \\
\hline Item 1.1* & -3.77 & -3.04 & -1.77 & -.37 & -3.77 & -3.04 & -1.77 & -.37 \\
\hline Item 1.2* & -3.88 & -3.09 & -1.69 & -.23 & -3.88 & -3.09 & -1.69 & -.23 \\
\hline Item 1.3 & -3.35 & -2.90 & -2.09 & -1.36 & -3.09 & -2.84 & -2.18 & -1.37 \\
\hline Item 1.4* & -2.59 & -2.00 & -.97 & .20 & -2.59 & -2.00 & -.97 & .20 \\
\hline Item 1.5 & -1.85 & -1.21 & -.12 & 1.01 & -1.96 & -1.23 & -.09 & 1.10 \\
\hline Item 1.6 & -2.42 & -1.86 & -.93 & .00 & -2.37 & -1.74 & -.86 & .20 \\
\hline \multicolumn{9}{|c|}{ Classroom management } \\
\hline Item 2.1 & -2.50 & -1.64 & -.58 & .76 & -2.50 & -1.57 & -.57 & .90 \\
\hline Item 2.2 & -2.38 & -1.28 & -.13 & 1.13 & -2.31 & -1.28 & -.10 & 1.32 \\
\hline Item 2.3 & -2.73 & -2.13 & -1.42 & -.63 & -2.81 & -2.23 & -1.46 & -.54 \\
\hline Item 2.4 & -2.14 & -1.65 & -.81 & .01 & -2.17 & -1.71 & -.91 & .04 \\
\hline Item $2.5^{*}$ & -2.57 & -1.71 & -.64 & .75 & -2.57 & -1.71 & -.64 & .75 \\
\hline Item $2.6^{*}$ & -2.58 & -1.29 & .04 & 1.76 & -2.58 & -1.29 & .04 & 1.76 \\
\hline Item $2.7^{*}$ & -2.80 & -1.82 & -.58 & .82 & -2.80 & -1.82 & -.58 & .82 \\
\hline \multicolumn{9}{|c|}{ Providing clear instruction } \\
\hline Item 3.1 & -2.22 & -1.83 & -1.03 & .02 & -2.56 & -2.07 & -1.21 & .06 \\
\hline Item 3.2 & -1.87 & -1.37 & -.40 & .60 & -2.13 & -1.51 & -.40 & .67 \\
\hline Item 3.3 & -2.33 & -1.44 & -.16 & 1.16 & -2.55 & -1.55 & -.20 & 1.18 \\
\hline Item 3.4 & -1.53 & -.95 & -.12 & .69 & -1.77 & -1.04 & -.11 & .73 \\
\hline Item 3.5 & -1.71 & -1.06 & .06 & 1.13 & -1.91 & -1.14 & .10 & 1.18 \\
\hline Item $3.6^{*}$ & -2.82 & -2.02 & -.80 & .54 & -2.82 & -2.02 & -.80 & .54 \\
\hline Item 3.7* & -3.45 & -2.60 & -1.42 & -.11 & -3.45 & -2.60 & -1.42 & -.11 \\
\hline Item 3.8 & -1.63 & -1.01 & -.07 & .91 & -1.77 & -1.07 & -.06 & .91 \\
\hline Item 3.9* & -1.87 & -1.22 & -.35 & .53 & -1.87 & -1.22 & -.35 & .53 \\
\hline Item 3.10 & -1.61 & -1.00 & -.16 & .67 & -1.71 & -1.02 & -.14 & .72 \\
\hline \multicolumn{9}{|c|}{ Challenging students } \\
\hline Item 4.1* & -3.49 & -3.08 & -2.39 & -1.52 & -3.49 & -3.08 & -2.39 & -1.52 \\
\hline Item $4.2 *$ & -3.29 & -2.80 & -1.79 & -.49 & -3.29 & -2.80 & -1.79 & -.49 \\
\hline Item 4.3 & -2.33 & -1.87 & -.90 & .13 & -2.22 & -1.61 & -.72 & .34 \\
\hline Item $4.4^{*}$ & -3.25 & -2.64 & -1.56 & -.27 & -3.25 & -2.64 & -1.56 & -.27 \\
\hline Item 4.5 & -1.62 & -1.16 & -.44 & .29 & -1.54 & -1.01 & -.27 & .41 \\
\hline \multicolumn{9}{|c|}{ Goal-oriented teaching } \\
\hline Item 5.1 & -1.96 & -1.43 & -.62 & .11 & -2.04 & -1.48 & -.70 & .10 \\
\hline Item 5.2* & -1.31 & -.56 & .46 & 1.37 & -1.31 & -.56 & .46 & 1.37 \\
\hline Item 5.3 & -1.35 & -.78 & .09 & .83 & -1.26 & -.65 & .28 & 1.04 \\
\hline Item 5.4* & -1.10 & -.30 & .76 & 1.57 & -1.10 & -.30 & .76 & 1.57 \\
\hline Item $5.5^{*}$ & -.92 & -.15 & .91 & 1.68 & -.92 & -.15 & .91 & 1.68 \\
\hline Item 5.6 & -1.96 & -1.47 & -.60 & .23 & -1.95 & -1.43 & -.56 & .34 \\
\hline
\end{tabular}

Note. Anchor items are marked with an asterisk (*) 


\section{Creating a safe climate}

In Table A.3 the results of the empty model are presented. The intraclass correlation coefficient shows that the variance explained by teacher clustering is approximately the same for both the pretest (23\%) and the posttest (22\%). The intercept at the pretest and posttest revealed that the overall teacher score on the 'creating a safe climate' factor did not change over time, as both $\gamma_{0}^{\text {pre }}$ and $\gamma_{0}^{\text {post }}$ were approximately zero. Figure A. 1 confirms this finding as approximately an equal number of teachers scored above and below the solid line, suggesting that overall students perceived the safe climate in their classroom to be the same at the pretest and posttest.

Table A.3. Empty model - Creating a safe climate

\begin{tabular}{|c|c|c|c|c|c|c|}
\hline & \multicolumn{3}{|c|}{ Pretest } & \multicolumn{3}{|c|}{ Posttest } \\
\hline & Estimate & SD & HPD & Estimate & SD & HPD \\
\hline \multicolumn{7}{|l|}{ Fixed effects- level 2} \\
\hline Intercept $\gamma_{0}^{\text {post }}$ & .01 & .04 & {$[-.06, .09]$} & -.02 & .04 & {$[-.09, .06]$} \\
\hline Random effects & & & & & & \\
\hline Level-2 variance $\sigma_{p r e}^{2}, \sigma_{p o s t}^{2}$ & .23 & .03 & [.18. .29] & .21 & .03 & {$[.16, .27]$} \\
\hline Level-1 variance $\tau_{\text {pre }}^{2}, \tau_{\text {post }}^{2}$ & .78 & .02 & {$[.74, .82]$} & .78 & .04 & {$[.70, .85]$} \\
\hline$\rho_{\text {pre }}, \rho_{\text {post }}$ & .23 & .02 & {$[.18, .27]$} & .22 & .02 & {$[.17, .26]$} \\
\hline Covariance pre-post $\sigma_{\beta}$ & .12 & .02 & {$[.07, .16]$} & & & \\
\hline Correlation pre-post & .52 & .08 & {$[.36, .69]$} & & & \\
\hline
\end{tabular}

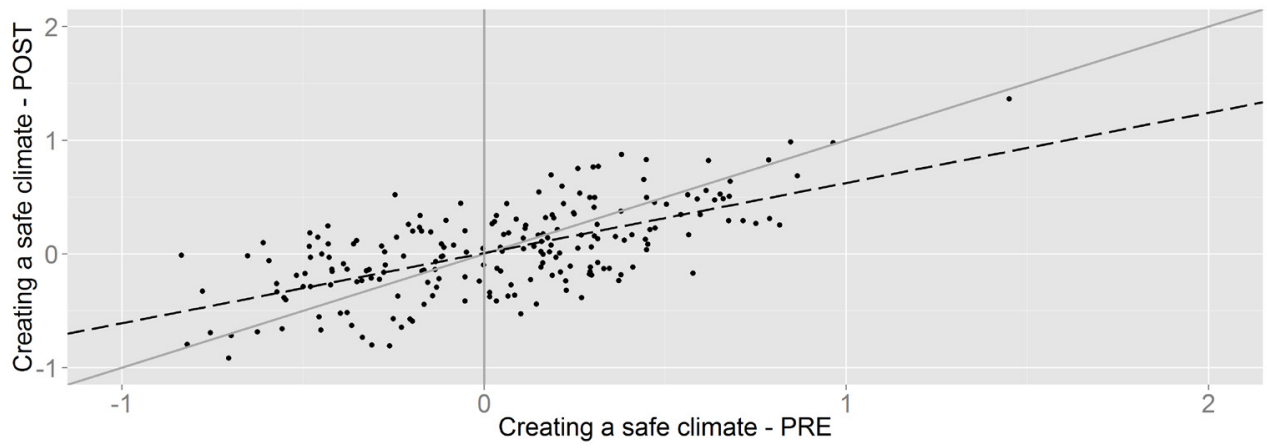

Figure A.1. Teachers' 'creating a safe climate' factor scores on pretest plotted against scores on posttest. The dotted line represents the linear regression of estimated pretest scores on posttest scores. The grey line represents the line of a perfect correlation between pre-scores and post-scores, and the vertical grey line represents the population-average pretest score of zero.

Whether a teacher transferred to a different grade, the gender of the teacher and whether 
the teacher taught a multigrade group did not influence the 'creating a safe climate' factor score $\left(\theta_{i j}^{\text {pre }}, \theta_{l j}^{\text {post }}\right)$. These explanatory variables were therefore excluded from the model. In the final model, shown in Table A.4, the explanatory factor variables 'grade', 'student gender' and 'teacher experience' were included. The level-1 explained proportions of variance $R_{1}^{2}$ was respectively .01 and .07 suggesting, that the included variables explained little variance within teachers. The level-2 explained proportion of variance $R_{2}^{2}$ was .12 for the pretest and .19 for the posttest.

Similarly to the empty model, no intervention effect was found in general, nor for a specific group of teachers. Results did show that students in grade 2 in both the pretest as well as the posttest gave significantly higher ratings to their teachers on 'creating a safe climate' compared to grade 6. Furthermore, girls, compared to boys, tended to be more positive about the climate in the classroom. Although the effect of student gender decreased significantly from pretest to posttest, at the posttest girls were still more positive (difference in effect:-.10, SD =.05, HPD [-.20,-.01]). Finally, the negative effect of teacher experience at both the pretest and posttest suggests that the older teachers become, the lower they are rated on 'safe classroom climate' by their students.

Table A.4. Final model - Creating a safe climate

\begin{tabular}{|c|c|c|c|c|c|c|}
\hline & \multicolumn{3}{|c|}{ Pretest } & \multicolumn{3}{|c|}{ Posttest } \\
\hline & Estimate & SD & HPD & Estimate & SD & HPD \\
\hline \multicolumn{7}{|l|}{ Fixed effects- level 2} \\
\hline Intercept & -.19 & .07 & {$[-.32,-.06]$} & -.16 & .08 & {$[-.32, .00]$} \\
\hline $\begin{array}{l}\text { Years of experience } \\
\text { (grand mean centered) }\end{array}$ & -.01 & .00 & {$[-.02,-.01]$} & -.01 & .00 & {$[-.02,-.01]$} \\
\hline \multicolumn{7}{|l|}{ Fixed effects - level 1} \\
\hline Grade 2 & .21 & .10 & {$[.02, .40]$} & .36 & .11 & {$[.14, .58]$} \\
\hline Grade 3 & .05 & .09 & {$[-.14, .23]$} & .12 & .11 & {$[-.11, .33]$} \\
\hline Grade 4 & .19 & .09 & {$[.00, .36]$} & -.02 & .10 & {$[-.21, .20]$} \\
\hline Grade 5 & -.02 & .09 & {$[-.20, .15]$} & .00 & .09 & {$[-.18, .17]$} \\
\hline Grade $6=$ ref & & & & & & \\
\hline Girl & .24 & .03 & {$[.18, .30]$} & .14 & .03 & {$[.07, .20]$} \\
\hline Boy $=$ ref & & & & & & \\
\hline$\underline{\text { Random effects }}$ & & & & & & \\
\hline Level-2 variance $\sigma_{p r e}^{2}, \sigma_{\text {post }}^{2}$ & .21 & .03 & {$[.16, .26]$} & .19 & .03 & {$[.14, .24]$} \\
\hline Level-1 variance $\tau_{p r e}^{2}, \tau_{p o s t}^{2}$ & .77 & .02 & {$[.73, .80]$} & .78 & .04 & {$[.69, .85]$} \\
\hline Intra class correlation $\rho_{\text {pre }}, \rho_{\text {post }}$ & .21 & .02 & {$[.17, .26]$} & .20 & .02 & {$[.16, .24]$} \\
\hline Covariance pre-post $\sigma_{\beta}$ & .10 & .02 & {$[.06, .14]$} & & & \\
\hline$\underline{\text { Correlation pre-post }}$ & .50 & .09 & {$[.33, .66]$} & & & \\
\hline
\end{tabular}




\section{Classroom Management}

In table A.5 the results of the empty model are presented. The intraclass correlation shows that at the pretest $32 \%$ of the variance was explained by the teacher clustering, at the posttest this was $29 \%$. The intercept at the posttest revealed a small but positive change from pretest to posttest suggesting that, according to students, overall teachers' classroom management skills slightly improved during the intervention. The graph presented in Figure A.2 shows that this was especially the case for teachers who scored below average at the pretest.

Table A.5. Empty model - Classroom management

\begin{tabular}{lllllll}
\hline & \multicolumn{3}{c}{ Pretest } & & \multicolumn{3}{c}{ Posttest } \\
& Estimate & SD & HPD & Estimate & SD & HPD \\
\hline
\end{tabular}

Fixed effects- level 2

Intercept $\gamma_{0}^{\text {post }}$

$.02 \quad .04 \quad[-.06, .10]$

.13

$.04 \quad[.04, .20]$

$\underline{\text { Random effects }}$

Level-2 variance $\sigma_{\text {pre }}^{2}, \sigma_{\text {post }}^{2}$

.33

$[.26, .40]$

$.26 \quad .03$

$[.20, .32]$

Level-1 variance $\tau_{\text {pre }}^{2}, \tau_{\text {post }}^{2}$

.69

$.02 \quad[.65, .72]$

$.65 \quad .04$

$[.58, .72]$

Intra class correlation $\rho_{\text {pre }}, \rho_{\text {post }}$

$.32 \quad .03 \quad[.27, .37]$

$.29 \quad .02$

$[.24, .34]$

\begin{tabular}{llll} 
Covariance pre-post & .12 & .03 & {$[.07, .17]$} \\
\hline Correlation pre-post & .40 & .08 & {$[.24, .56]$} \\
\hline
\end{tabular}

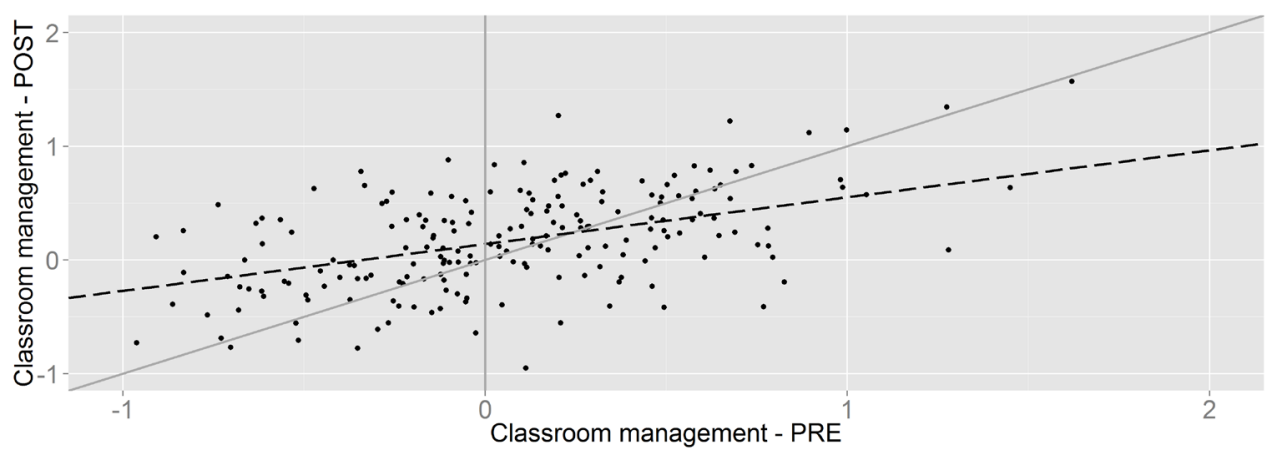

Figure A.2. Teachers' 'classroom management' factor scores on pretest plotted against scores on posttest. the dotted line represents the linear regression of estimated pretest scores on posttest scores. The grey line represents the line of a perfect correlation between pre-scores and post-scores, and the vertical grey line represents the population-average pretest score of zero.

The covariates 'student gender', 'teacher gender', 'years of experience' and 'multigrade group' did not influence students' classroom management perceptions and therefore were 
excluded from the model. In the final model (Table A.6) only the explanatory variables 'grade' and whether a teacher changed grades from pretest to posttest were included. The level-1 explained proportions of variance $R_{1}^{2}$ were respectively .00 and -.01 suggesting that the included variables explained no variance within teachers. The included background variables did explain level-2 variance: the explained proportion of variance $R_{2}^{2}$ was .14 for the pretest and .17 for the posttest.

The overall positive intervention effect, found in the empty model, faded when including the background variables. This might have been caused by the fact that students in grade 2 rated their teacher significantly higher during both the pretest and posttest compared to the other grades. Additionally, according to their students, teachers who changed groups had slightly higher classroom management skills at both the pretest as well as the posttest when compared to teachers who remained in the same group.

Table A.6. Final model - Classroom management

\begin{tabular}{|c|c|c|c|c|c|c|}
\hline & \multicolumn{3}{|c|}{ Pretest } & \multicolumn{3}{|c|}{ Posttest } \\
\hline & Estimate & SD & HPD & Estimate & SD & HPD \\
\hline \multicolumn{7}{|l|}{ Fixed effects- level 2} \\
\hline Intercept & .17 & .08 & {$[-.32, .01]$} & -.09 & .08 & {$[-.24, .06]$} \\
\hline Changed grade & .17 & .08 & {$[.01, .32]$} & .20 & .08 & {$[.05, .35]$} \\
\hline \multicolumn{7}{|l|}{ Remained in the same grade $=$ ref } \\
\hline \multicolumn{7}{|l|}{ Fixed effects - level 1} \\
\hline Grade 2 & .50 & .10 & {$[.30, .70]$} & .58 & .10 & {$[.38, .79]$} \\
\hline Grade 3 & .08 & .10 & {$[-.13, .27]$} & .15 & .10 & {$[-.05, .34]$} \\
\hline Grade 4 & .10 & .10 & {$[-.10, .29]$} & -.07 & .10 & {$[-.27, .11]$} \\
\hline Grade 5 & -.07 & .09 & {$[-.25, .09]$} & .03 & .08 & {$[-.13, .18]$} \\
\hline \multicolumn{7}{|l|}{ Grade $6=$ ref } \\
\hline \multicolumn{7}{|l|}{$\underline{\text { Random effects }}$} \\
\hline Level-2 variance $\sigma_{p r e}^{2}, \sigma_{p o s t}^{2}$ & .28 & .03 & {$[.22, .35]$} & .22 & .03 & {$[.16, .28]$} \\
\hline Level-1 variance $\tau_{p r e}^{2}, \tau_{p o s t}^{2}$ & .69 & .02 & {$[.65, .72]$} & .66 & .04 & {$[.58, .73]$} \\
\hline Intra class correlation $\rho_{\text {pre }}, \rho_{\text {post }}$ & .29 & .03 & {$[.24, .34]$} & .25 & .02 & {$[.20, .30]$} \\
\hline Covariance pre-post $\sigma_{\beta}$ & .09 & .02 & {$[.05, .13]$} & & & \\
\hline Correlation pre-post & .35 & .08 & {$[.18, .50]$} & & & \\
\hline
\end{tabular}

\section{Providing Clear Instruction}

In Table A.7 the results of the empty model are presented. The intraclass correlation coefficient shows that at both the pretest and the posttest $19 \%$ of the variance was explained by the teacher clustering. The intercept at the pretest and the posttest were both approximately zero, suggesting that no changes in student perceptions occurred pertaining to the clearness of teachers' instruction. Figure A.3 confirms this finding, as approximately an equal number of teachers scored above and below the solid line. 
Table A.7. Empty model - Providing clear instruction

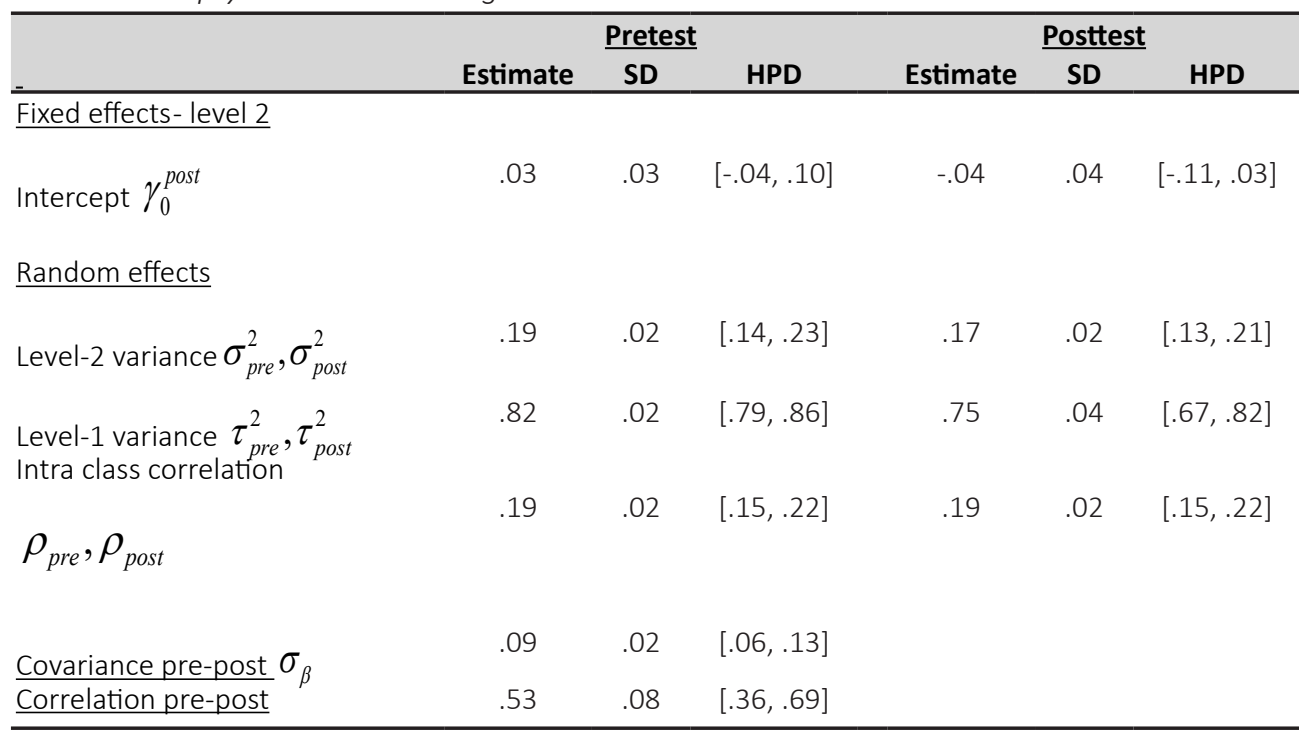

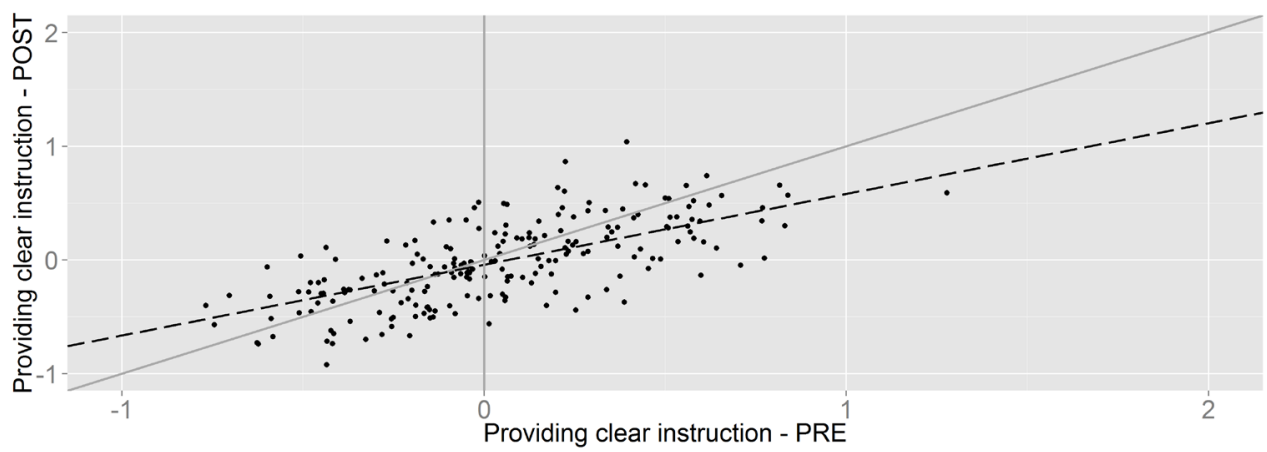

Figure A.3. Teachers' 'providing clear instruction' factor scores on pretest plotted against scores on posttest. The dotted line represents the linear regression of estimated pretest scores on posttest scores. The grey line represents the line of a perfect correlation between pre-scores and post-scores, and the vertical grey line represents the population-average pretest score of zero.

Covariates 'teacher gender', 'multigrade group' and whether or not teachers changed grades did not influence students' perceptions of the instruction they received and were therefore excluded from the final model. In the final model (Table A.8) 'grade', 'student gender', and 'years of experience' were included as explanatory variables. The level-1 explained proportion of variance $R_{1}^{2}$ was respectively .01 and .00 suggesting that the included variables explained no variance within teachers. The level- 2 explained proportion of variance $R_{2}^{2}$ was .08 for the pretest, and .14 for the posttest.

Interestingly, student grade did not influence students' perceptions of instruction at the start of the intervention. At the posttest teachers in grade 2 scored significantly higher, and teachers in grade 4 significantly lower, compared to grade 6 . Furthermore, at the pretest girls reported being more positive about their teacher with regards to instruction. This 
significantly decreased at the posttest resulting in the same perceptions by boys and girls at this point. A small but significant effect of years of experience was found at the pretest suggesting that students were less positive about the instruction of older teachers. Note that teachers' years of experienced varied from 1 year to 40 years, as a consequence the effect could vary from .16 for students with 1 year of experience and- .24 for teachers with 40 years of experience.

Table A.8. Final model - Providing clear instruction

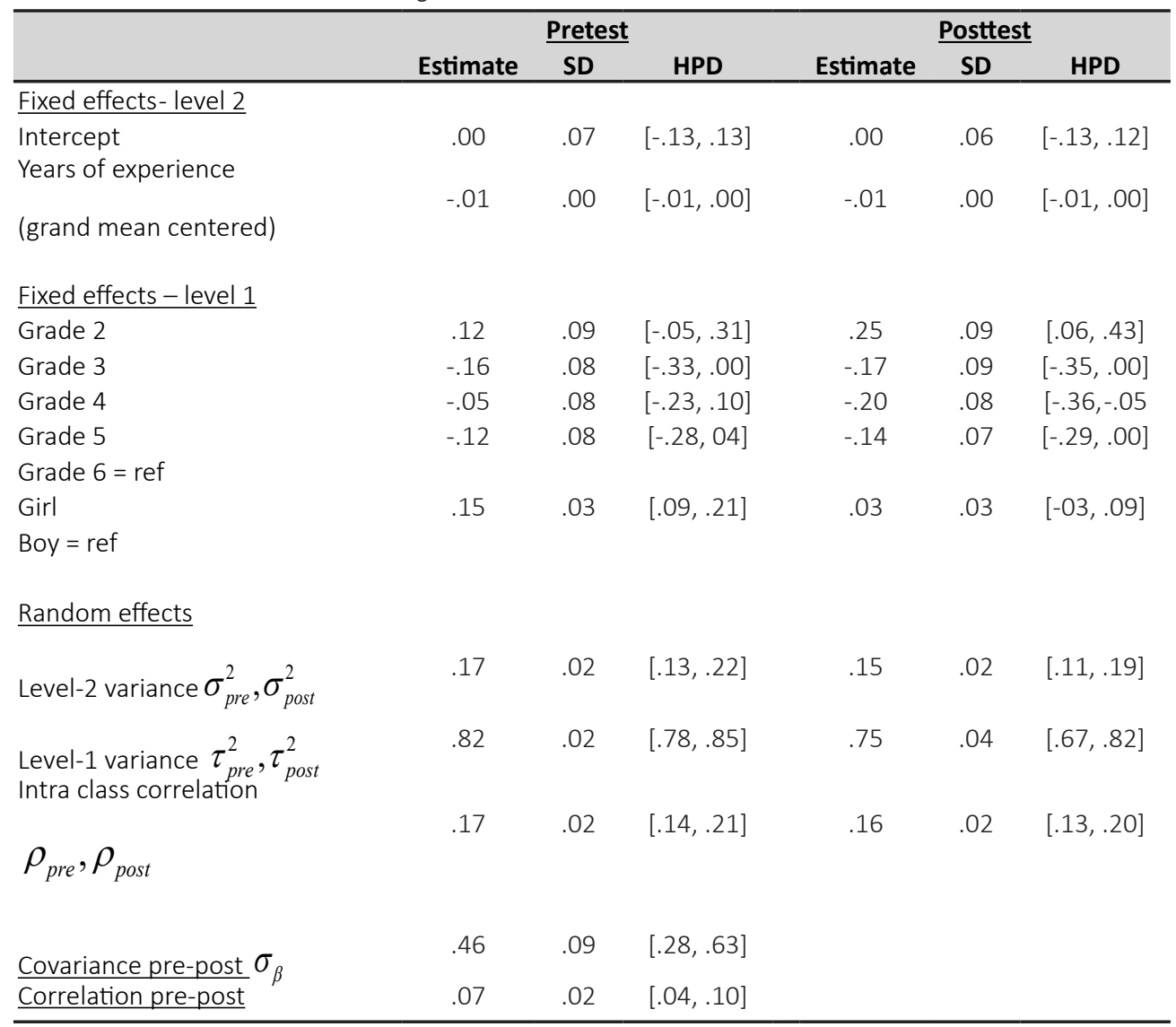

\section{Challenging Students}

In Table A.9 the results of the empty model are presented. The intraclass correlation shows that at both the pretest and the posttest around $20 \%$ of the variance was explained by the teacher clustering. The intercept at the posttest of .21 suggests an overall significant change from pretest to posttest, without controlling for background variables. On average the population average score on the 'challenging students' scale is significantly higher on the posttest compared to the pretest. In Figure A.4 it can be seen that most of the teachers (scoring below or above average at the pretest), scored higher at the posttest. 
Table A.9. Empty model - Challenging students

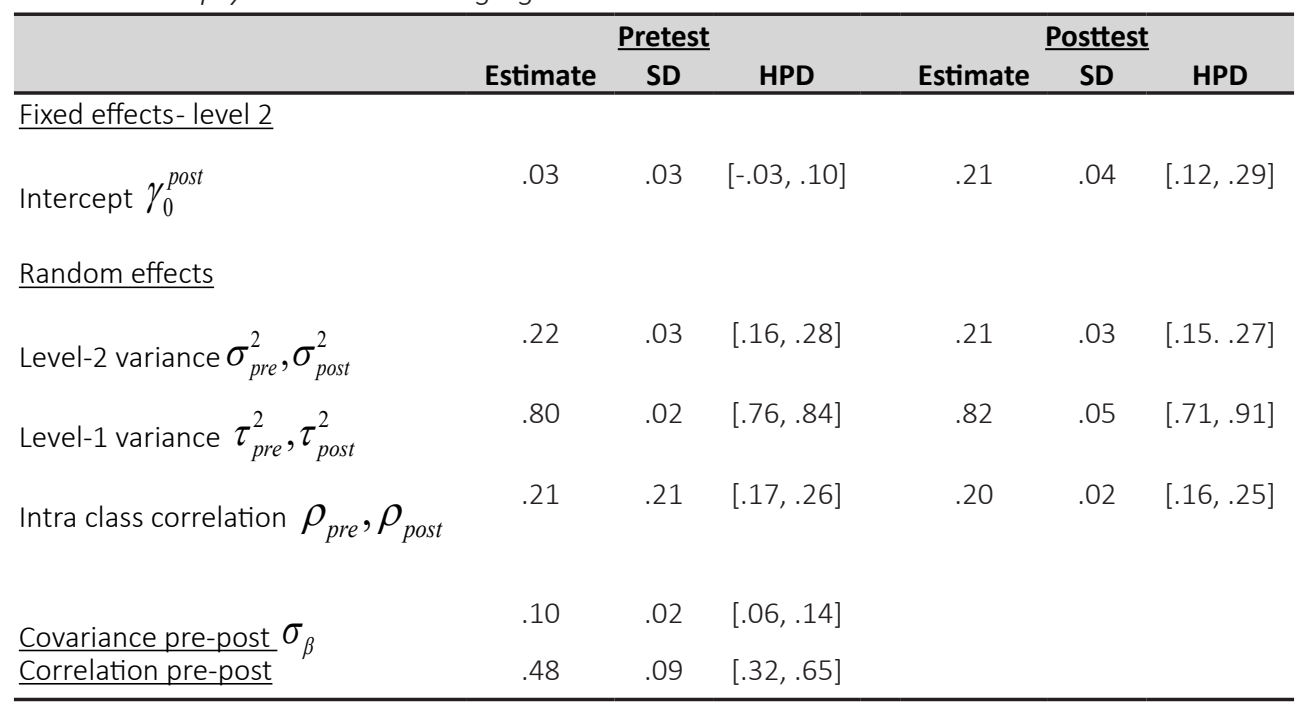

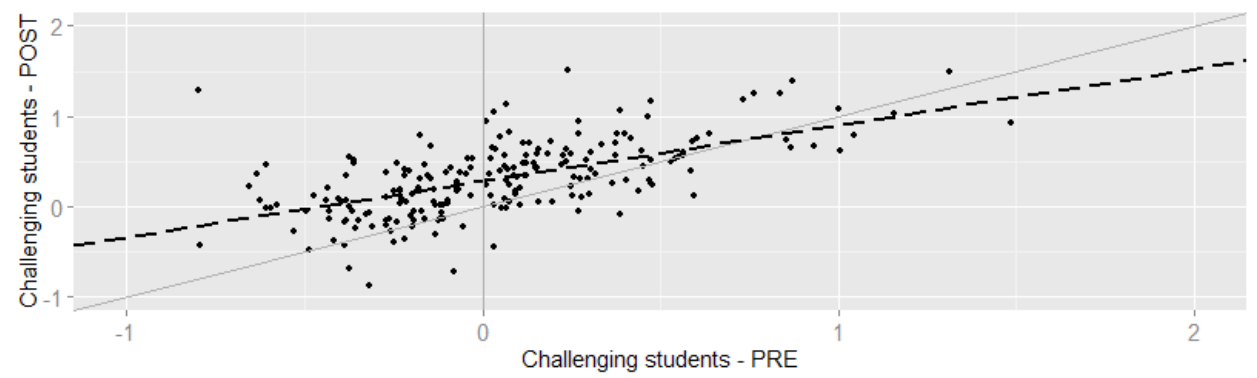

Figure A.4. Teachers' 'challenging students' factor scores on pretest plotted against scores on posttest. The dotted line represents the linear regression of estimated pretest scores on posttest scores. The grey line represents the line of a perfect correlation between pre-scores and post-scores, and the vertical grey line represents the population-average pretest score of zero.

The variables 'teachers years of experience', 'teachers' gender', and whether or not a teacher changed grades during the school year did not influence the score on the 'challenging students' scale. These variables therefore were excluded from the final model. Table A.10 shows the results of the final model, with the covariates 'grade', 'student gender' and 'multigrade group'. The level-1 explained proportion of variance $R_{1}^{2}$ was respectively .01 and -.02 suggesting that the included variables explained no variance within teachers. The level-2 explained proportion of variance $R_{2}^{2}$ was .18 for the pretest and .17 for the posttest.

At both the pretest and the posttest students in grade 2 were more positive about their teachers compared to grade 6 students. At the pretest students in grade 3 were also significantly more positive about their teachers. At the posttest girls were more positive than boys however this effect disappeared at the posttest. Teachers in multigrade groups scored significantly lower on 'challenging students'. This effect was not present anymore at the 
posttest. For these teachers, the effect of the intervention was the largest (intervention effect $=.43, S D=.15, \operatorname{HPD}[.13, .70])$. For all teachers, other than those in grade 3 , the intervention has had a significant positive effect.

Table A.10. Final model - Challenging students

\begin{tabular}{|c|c|c|c|c|c|c|}
\hline & \multicolumn{3}{|c|}{ Pretest } & \multicolumn{3}{|c|}{ Posttest } \\
\hline & Estimate & SD & HPD & Estimate & SD & HPD \\
\hline \multicolumn{7}{|l|}{ Fixed effects- level 2} \\
\hline Intercept & -.14 & .07 & {$[-.29, .27]$} & .13 & .08 & {$[-.01, .27]$} \\
\hline Multigrade group & -.16 & .09 & {$[-.35, .00]$} & .00 & .09 & {$[-.17, .18]$} \\
\hline \multicolumn{7}{|l|}{ Non-multigrade $=$ ref } \\
\hline \multicolumn{7}{|l|}{ Fixed effects - level 1} \\
\hline Grade 2 & .53 & .10 & {$[.33, .73]$} & .59 & .11 & {$[.39, .79]$} \\
\hline Grade 3 & .21 & .09 & {$[.02, .39]$} & .17 & .10 & {$[-.03, .36]$} \\
\hline Grade 4 & .11 & .09 & {$[-.08, .29]$} & .00 & .09 & {$[-.18, .16]$} \\
\hline Grade 5 & -.02 & .08 & {$[-.18, .15]$} & -.04 & .08 & {$[-.20, .12]$} \\
\hline Grade $6=$ ref & & & & & & \\
\hline Girl & .09 & .03 & {$[.02, .15]$} & -.05 & .04 & {$[-.13, .02]$} \\
\hline Boy $=r e f$ & & & & & & \\
\hline \multicolumn{7}{|l|}{$\underline{\text { Random effects }}$} \\
\hline Level-2 variance $\sigma_{p r e}^{2}, \sigma_{\text {post }}^{2}$ & .18 & .02 & {$[.14, .23]$} & .17 & .03 & {$[.12, .23]$} \\
\hline Level-1 variance $\tau_{\text {pre }}^{2}, \tau_{\text {post }}^{2}$ & .80 & .02 & {$[.76, .84]$} & .83 & .06 & {$[.71, .93]$} \\
\hline Intra class correlation $\rho_{\text {pre }}, \rho_{\text {post }}$ & .18 & .02 & {$[.14, .22]$} & .17 & .02 & {$[.13, .21]$} \\
\hline Covariance pre-post $\sigma_{\beta}$ & .45 & .10 & {$[.26,63]$} & & & \\
\hline Correlation pre-post & .08 & .02 & {$[.04, .12]$} & & & \\
\hline
\end{tabular}




\section{Appendix D}

\section{Questionnaire Leadership for DBDM}

All items were scored on a four-point Likert scale, ranging from 'totally disagree' to 'totally agree'.

The school leader ...

1. ... takes time to talk with teachers to discuss problems they encounter with students

2. ... conducts classroom observations on a yearly basis

3. ... ensures that team meetings are valuable meetings about education

4. ... ensures that the school climate is learning oriented

5. ... shows appreciation for teachers who do a good job

6. ... discusses problems with regard to teaching timely with the people who are involved

7. ... shows interest in what happens inside the classrooms

8. ... stresses the need to regularly monitor student achievement

9. ... stimulates teachers to maximize learning achievement

10. ... guides and supports teachers in maximizing student achievement

11. ... stimulates cooperation between teachers

12. ... ensures a shared feeling of responsibility for the achievement of all students

13. ... promotes that lessons are of high quality 


\section{Appendix E}

\section{Questionnaire Data Culture}

All items were scored on a four-point Likert scale, ranging from 'totally disagree' to 'totally agree'.

In our school ...

1. ... we value student achievement for core subjects (mathematics, reading and language) higher than achievement in other subjects

2. ... we think it is important that our score at the standardized end-of-school-test is higher than that of comparable schools

3. ... it is regularly stressed that it is important to set ambitious goals for all students

4. ... we think we can reach ambitious goals for all students, taking their capabilities into account

5. ... all teachers think that you can improve achievement by putting more effort in it

6. ... teachers have high but realistic expectations of all students

7. ... students feel their teachers have high expectations with regard to achievement

8. ... the student monitoring system is used at least twice a year to monitor student achievement in all grades

9. ... we discuss student achievement data from the student monitoring system as a team

10. ... we adapt our education for each student, based on their achievement 


\section{Appendix F}

\section{R Script of the Multivariate Linear Analysis Using the Car Package and}

\section{Contrast Matrices to Test Hypotheses}

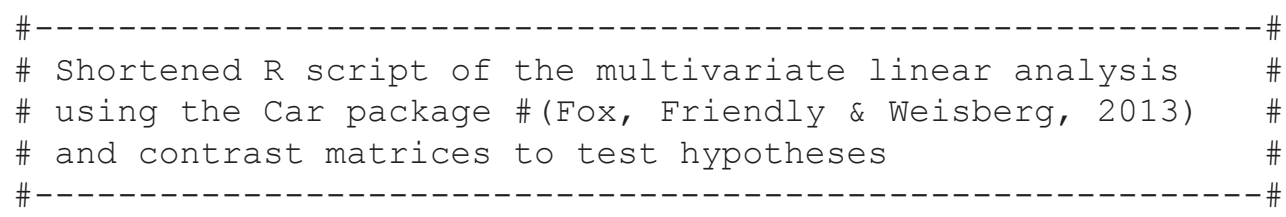

\#\# Test de within-subject factors type and time (using the \#Car package)

manova.centr<-Anova (mod.centr, idata=idata, idesign= Type*Time, test.statistic="Wilks", Type=3)

\# The TestContrast() function can be used to test user-defined \#contrast (s)

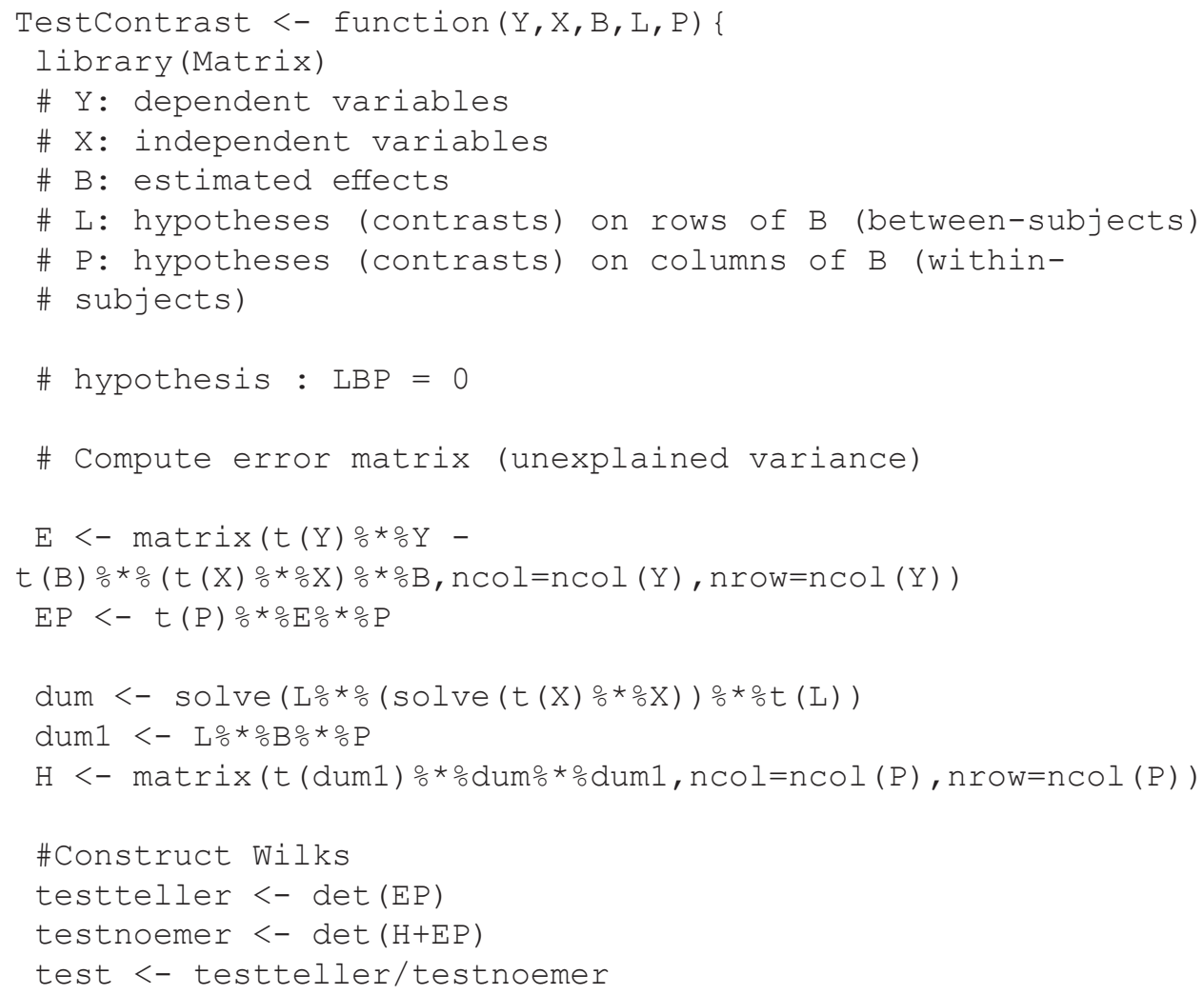


\#\# $q$ : : number of columns in predictor $\mathrm{X}$ used in \#constructing contrast

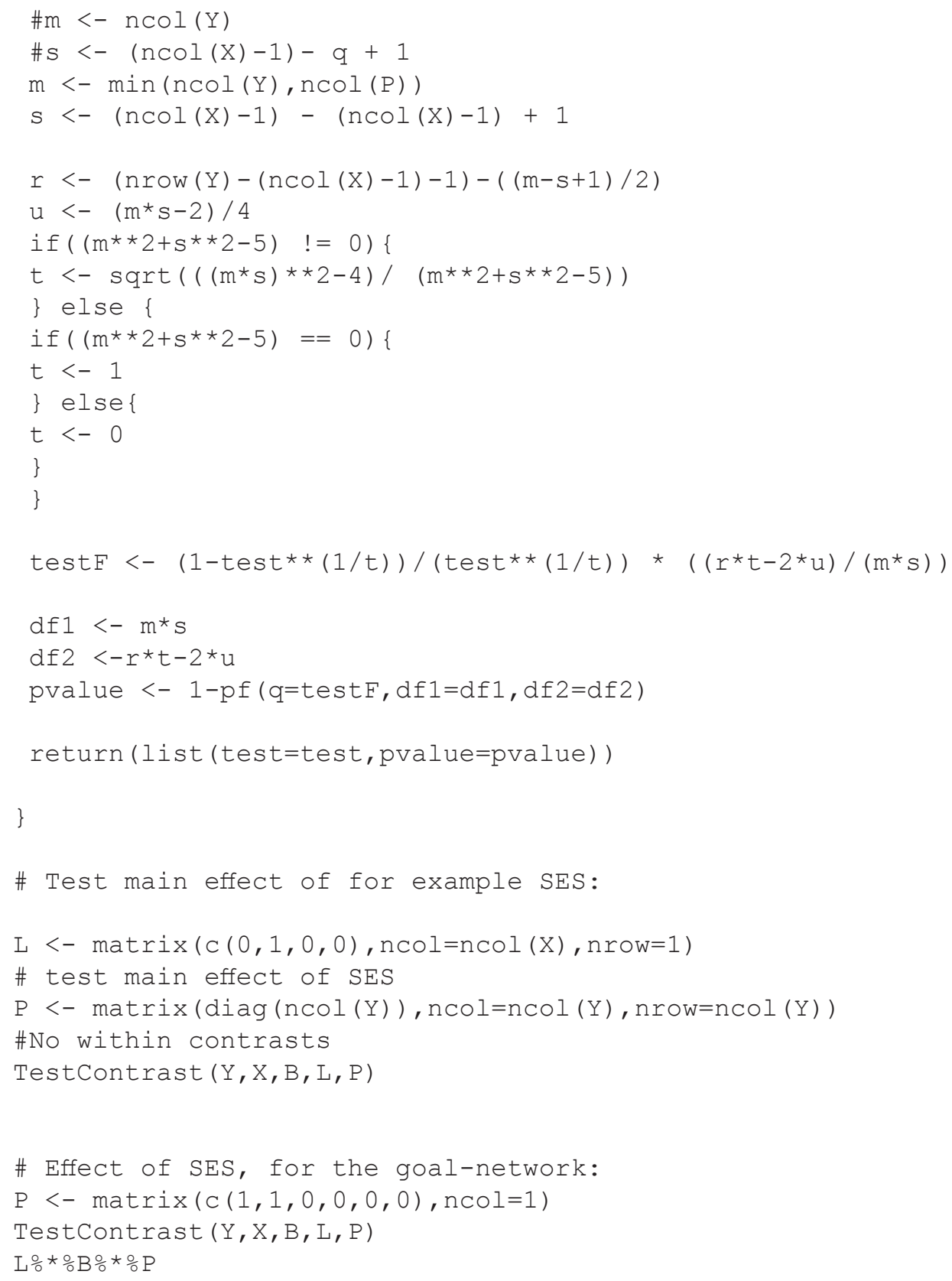




\section{Appendix G}

\section{Questionnaires to measure educators' attitude}

\section{Attitude towards DBDM in general}

To monitor and improve the results of my/our teaching, data-based decision making is...
1. Advisable

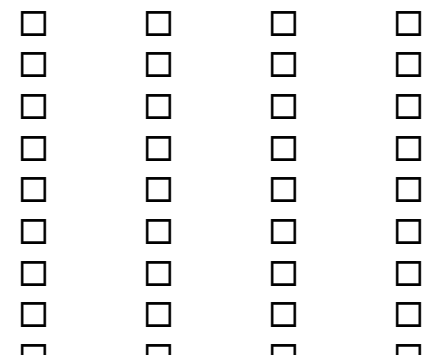
Not advisable
2. Good
$\begin{array}{ll}\square & \text { Bad } \\ \square & \text { Unsuitable }\end{array}$
3. Suitable
4. Usable
Unusable
5. Pleasant
Unpleasant
6. Valuable
Valueless
7. Motivating
Demotivating
8. Meaningful
Meaningless
9. Easy
Difficult
10. Positive
Negative
11. Desirable
Undesirable
12. Important
Unimportant

\section{Attitude towards analyzing student achievement data with the student monitoring system}

Items were scored on a 1-4 scale, ranging from 'totally disagree' to 'totally agree'

Using the student monitoring system..

13. ... is essential for realizing positive student outcomes

14. ... is crucial to determine how we can achieve better student outcomes

15. ... requires the provision of adaptive education for all students

16. ...is necessary to correctly determine where our education fails

17. ... is an activity in which I would like to invest more time

\section{Attitude towards setting goals based on data}

Items were scored on a 1-4 scale, ranging from 'totally disagree' to 'totally agree'

18. Formulating challenging performance goals for subsequent SMS-tests leads to higher student achievement compared to working without such performance goals

19. Without precise performance goals for subsequent SMS-tests, I cannot achieve as high student outcomes as with such goals

20. The continuous pressure to formulate performance goals for the next SMS-tests, negatively affects my motivation.

21. Setting performance goals puts too much emphasis on students' academic achievement. 


\section{Attitude towards collaboration during the DBDM process}

Items were scored on a 1-4 scale, ranging from 'totally disagree' to 'totally agree'

By collaborating as a school team...

22. ... we achieve better student achievement

23. ... an unpleasant pressure to achieve high performance increasess

24. ... we learn from each other

25. ... we function better as a team

26. ... we lose valuable teaching time

Other items divided over the previous four scales based on a confirmatory factor analysis Items were scored on a 1-4 scale, ranging from 'totally disagree' to 'totally agree'

How desirable are the following matters in the context of DBDM according to you?

27. The use of the student monitoring system

28. Formulating student performance goals, based on test results

29. Collaboration within your team

Item 27 was included in the scale 'attitude towards analyzing student achievement data with the student monitoring system'; item 28 was included in the scale 'attitude towards setting goals based on data'; item 29 was included in the scale 'attitude towards collaboration during the DBDM process'. 


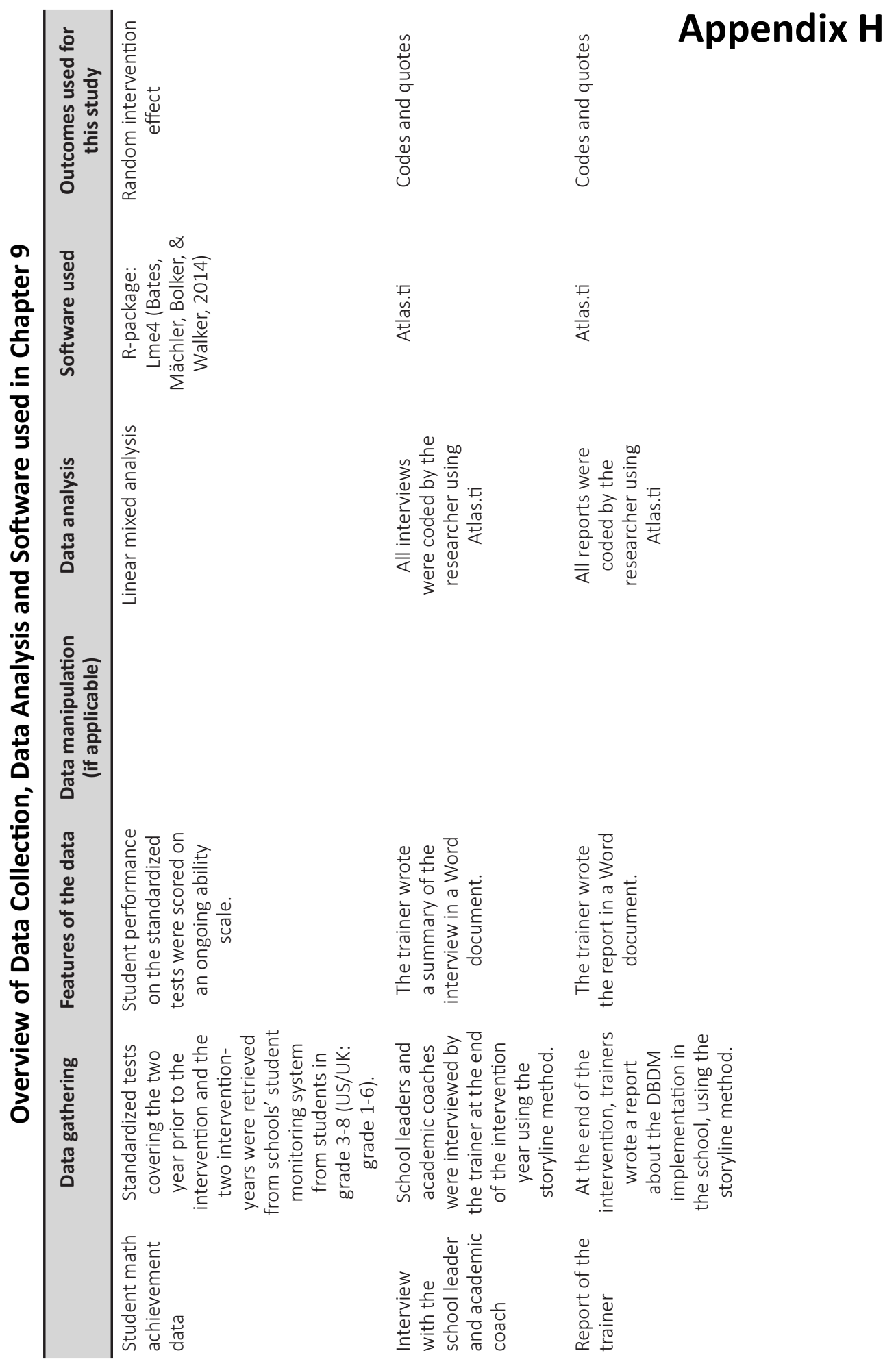




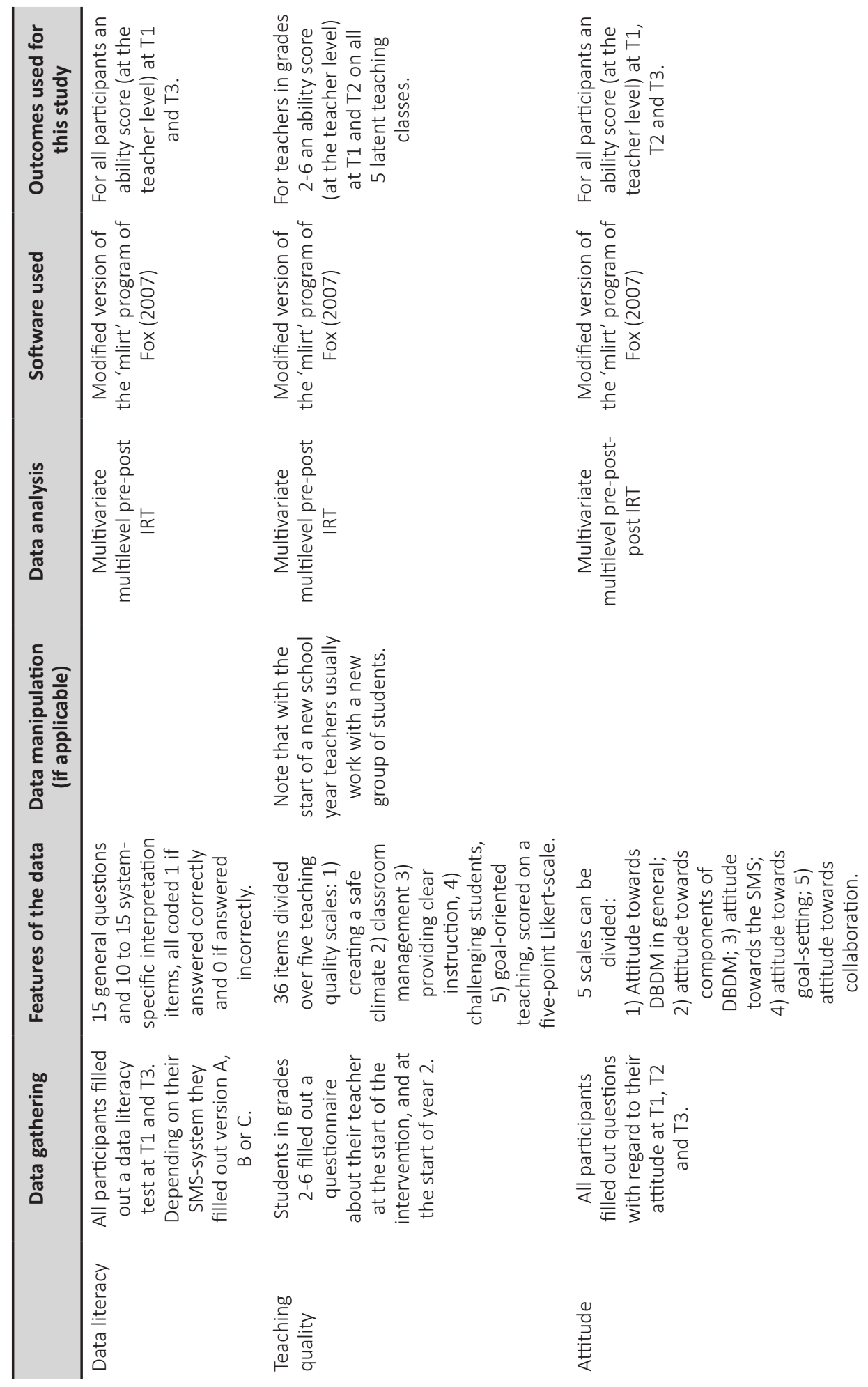




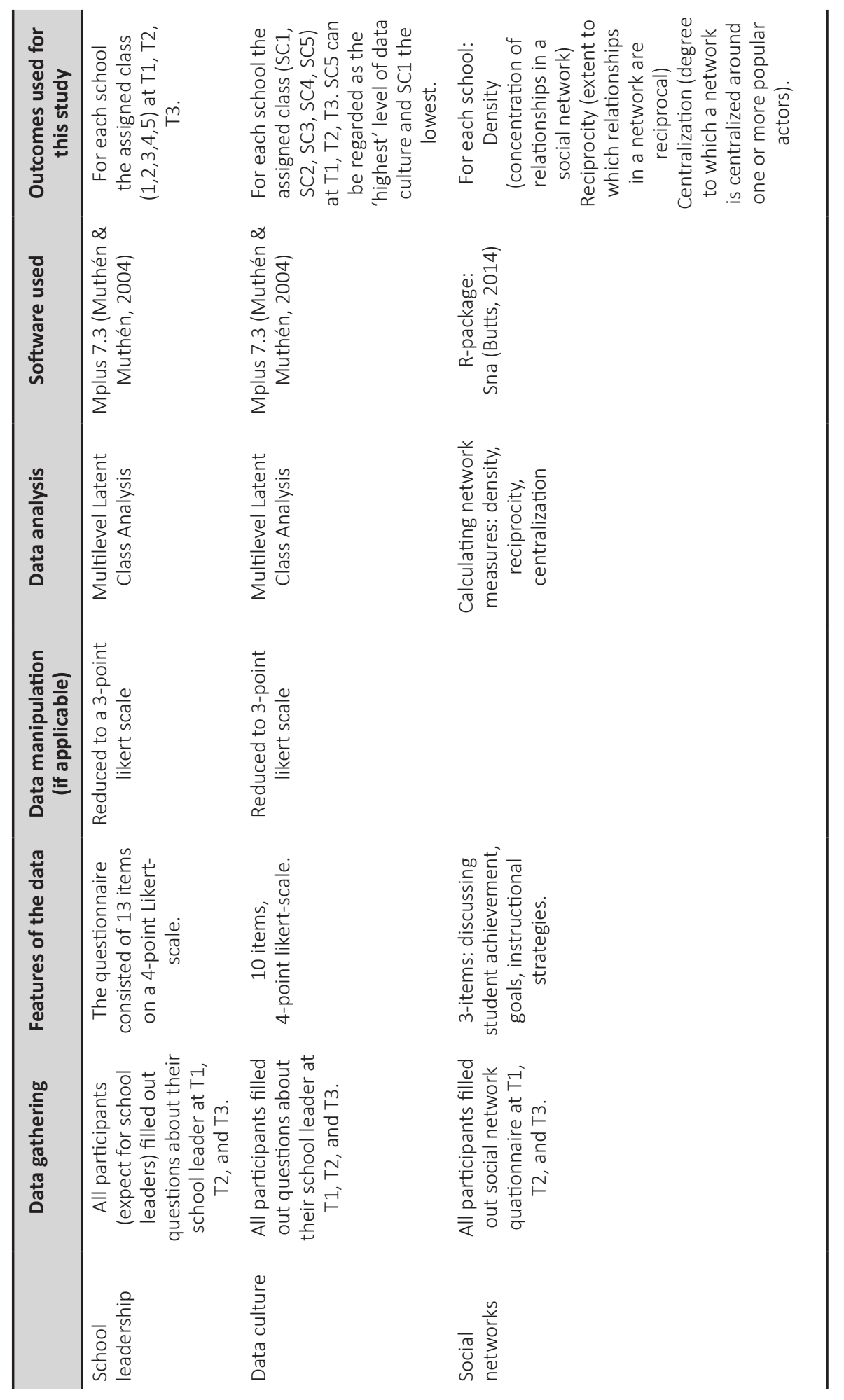




\section{References}

Allison, P. D. (1990). Change scores as dependent variables in regression analysis. Sociological Methodology, 20, 93-114.

Anderson, S., Leithwood, K., \& Strauss, T. (2010). Leading data use in schools: Organizational conditions and practices at the school and district levels. Leadership and Policy in Schools, 9(3), 292-327. http://doi.org/10.1080/15700761003731492

Asparouhov, T., \& Muthen, B. (2013). Auxiliary variables in mixture modeling: 3-Step approaches using Mplus. Mplus Web Notes: No. 15, 1-48. Retrieved from http://www.statmodel.com/examples/ webnotes/webnote15.pdf

Atteberry, A., \& Bryk, A. S. (2010). Centrality, connection, and commitment: The role of social networks in a school-based literacy initiative. In A. J. Daly (Ed.), Social network theory and educational change (pp. 51-75). Cambridge, MA: Harvard University Press.

Azevedo, C. L. N., Andrade, D. F., \& Fox, J.-P. (2012). A Bayesian generalized multiple group IRT model with model-fit assessment tools. Computational Statistics and Data Analysis, 56(12), 43994412. http://doi.org/10.1016/j.csda.2012.03.017

Balch, R. T. (2012). The validation of a student survey on teacher practice (Doctoral dissertation, Vanderbilt University).

Bates, D., Mächler, M., Bolker, B., \& Walker, S. (2014). Fitting linear mixed-effects models using Ime4. arXiv:1406.5823 [stat.CO].

Beijaard, D., Driel van, J., \& Verloop, N. (1999). Evaluation of story-line methodology in research on teachers' practical knowledge. Studies in Educational Evaluation, 25(99), 47-62.

Bennett, R. E. (2011). Formative assessment: A critical review. Assessment in Education: Principles, Policy \& Practice, 18(1), 5-25. http://doi.org/10.1080/0969594X.2010.513678

Bennink, M., Croon, M. A., \& Vermunt, J. K. (2013). Micro-macro multilevel analysis for discrete data: A latent variable approach and an application on personal network data. Sociological Methods \& Research, 42(4), 431-457. http://doi.org/10.1177/0049124113500479

Berends, B. Y. M., Bodilly, S. J., \& Kirby, S. N. (2002). Looking back over a decade of whole-school reform: The experience of new American schools. Phi Delta Kappan, 84(2), 168-175.

Bernhardt, V. L. (2005). Data tools for school improvement. Educational Leadership, 62(5), 66-69.

Bijmolt, T. H. a, Paas, L. J., \& Vermunt, J. K. (2004). Country and consumer segmentation: Multilevel latent class analysis of financial product ownership. International Journal of Research in Marketing, 21, 323-340. http://doi.org/10.1016/j.ijresmar.2004.06.002

Bill \& Melinda Gates Foundation. (2010). Learning about teaching: Initial findings from the Measures of Effective Teaching project. Seattle, WA. Retrieved from http://www.metproject.org/downloads/ Preliminary_Findings-Research_Paper.pdf

Bill \& Melinda Gates Foundation. (2012). Asking students about teaching: Student perceptions surveys and their implementation. Retrieved from http://www.metproject.org/downloads/Asking Students_Practitioner_Brief.pdf

Birman, B. F., Desimone, L. M., Porter, A. C., \& Garet, M. S. (2000). Designing professional development that works. Educational Leadership, 57(8), 28-33.

Black, B. P., \& Wiliam, D. (1998). Inside the black box: Raising standards through classroom assessment. Assessment in Education, 5(1), 7-74.

Bock, R. D., \& Zimowski, M. F. (1997). Multiple group IRT. In W. J. van der Linden \& R. K. Hambleton (Eds.), Handbook of modern item response theory (pp. 433-448). New York, NY: Springer.

Borgatti, S. P., \& Foster, P. C. (2003). The network paradigm in organizational research: A review 
and typology. Journal of Management, 29(6), 991-1013. http://doi.org/10.1016/S01492063(03)00087-4

Borgatti, S. P., \& Ofem, B. (2010). Overview: Social network theory and analysis. In A. J. Daly (Ed.), The ties of change: Social network eduction (pp. 17-30). Cambridghe, MA: Harvard Press.

Borgatti, S. P., Everett, M. G., \& Freeman, L. C. (2002). UCINET for Windows: Software for social network analysis. Harvard, MA: Analytic Technologies.

Boudett, K. P., City, E., \& Murnane, R. (2005). Data Wise: A step-by-step guide to using assessment results to improve teaching and learning. Cambridge, MA: Harvard Education Press.

Bron, R., Geel, M. van, \& Visscher, A. J. (2013). Opbrengstgericht werken op de pabo [Data-based decision making in teacher training institutions]. Zoetermeer, The Netherlands. Retrieved from http:// www.kennisnet.nl/uploads/tx_kncontentelements/Conceptverslag_opbrengstgerichtwerken_ op_de_pabo_-_4maart2013.pdf

Brophy, J. (2000). Teaching. (Educational practice Series, Ed.) (1st ed.). Switzerland, Lausanne: PCL.

Butts, C. T. (2014). sna: Tools for social network analysis. R package version 2.3-2. Retrieved from http:// cran.r-project.org/package=sna

Campbell, C., \& Levin, B. (2008). Using data to support educational improvement. Educational Assessment, Evaluation and Accountability, 21(1), 47-65. http://doi.org/10.1007/s11092-0089063-x

Carlson, D., Borman, G. D., \& Robinson, M. (2011). A multistate district-level cluster randomized trial of the impact of data-driven reform on reading and mathematics achievement. Educational Evaluation and Policy Analysis, 33(3), 378-398. http://doi.org/10.3102/0162373711412765

Cheung, A. C. K., \& Slavin, R. E. (2015). How methodological features affect effect sizes in education. Baltimore, MD.

Cito. (2009a). Rekenen-Wiskunde handleiding. Arnhem.

Cito. (2009b). Spelling handleiding. Arnhem.

Coburn, C. E., \& Russell, J. L. (2008). District policy and teachers' social networks. Educational Evaluation and Policy Analysis, 30(3), 203-235. http://doi.org/10.3102/0162373708321829

Coburn, C. E., \& Turner, E. O. (2011). Research on data use: A framework and analysis. Measurement: Interdisciplinary Research \& Perspective, 9(4), 173-206. http://doi.org/10.1080/15366367.201 1.626729

Coburn, C. E., \& Turner, E. O. (2012). The practice of data use: An introduction. American Journal of Education, 118(2), 99-111. http://doi.org/10.1086/663272

Coburn, C. E., Choi, L., \& Mata, W. (2010). "I would go to her because her mind is math": Network formation in the context of mathematics reform. In A. Daly (Ed.), Social network theory and educational change (pp. 33-50). Cambridge: Harvard Educational Press.

Coe, R. (2002). Evidence on the role and impact of performance feedback in schools. In A. J. Visscher \& R. Coe (Eds.), School improvement through performance feedback (pp. 3-26). Routledge.

Copland, M. A., Knapp, M. S., \& Swinnerton, J. A. (2009). Principal leadership, data, and school improvement. In T. J. Kowalski \& T. J. Lasley (Eds.), Handbook of data-based decision making in education (pp. 153-171). New York: Routledge.

Cornelius-White, J. (2007). Learner-centered teacher-student relationships are effective: A meta-analysis. Review of Educational Research, 77(1), 113-143. http://doi.org/10.3102/003465430298563

Cosner, S. (2012). Leading the ongoing development of collaborative data practices: Advancing a schema for diagnosis and intervention. Leadership and Policy in Schools, 11(1), 26-65. http:// doi.org/10.1080/15700763.2011.577926

Creemers, B., Kyriakides, L., \& Antoniou, P. (2013). Teacher Professional Development for Improving Quality of Teaching. Dordrecht: Springer Netherlands. http://doi.org/10.1007/978-94-007- 
5207-8

Cronbach, L., \& Meehl, P. (1955). Construct validity in psychological tests. Psychological Bulletin, 129, 3-9. http://doi.org/10.1037/h0040957

Cummings, J. N., \& Cross, R. (2003). Structural properties of work groups and their consequences for performance. Social Networks, 25, 197-210. http://doi.org/10.1016/S0378-8733(02)00049-7

Daly, A. J. (2012). Data, dyads, and dynamics: Exploring data use and social networks in educational improvement. Teachers College Record, 114(11), 1-38.

Daly, A. J., \& Finnigan, K. S. (2011). The ebb and flow of social network ties between district leaders under high-stakes accountability. American Educational Research Journal, 48(1), 37-79. http:// doi.org/10.3102/0002831210368990

Daly, A. J., Moolenaar, N. M., Bolivar, J. M., \& Burke, P. (2010). Relationships in reform: The role of teachers' social networks. Journal of Educational Administration, 48(3), 359-391. http://doi. org/10.1108/09578231011041062

Daniel, L. G., \& King, D. A. (1998). Knowledge and use of testing and measurement literacy of elementary and secondary teachers. The Journal of Educational Research, 91(6), 331-344.

Danielson, C. (2007). Enhancing professional practice: A framework for teaching (2nd ed.). Alexandria, VA: ASCD.

Darling-Hammond, L. (2000). Teacher quality and student achievement: A review of state policy evidence. Education Policy Analysis Archives, 8, 1. http://doi.org/10.1038/sj.clp

Datnow, A. (2011). Collaboration and contrived collegiality: Revisiting hargreaves in the age of accountability. Journal of Educational Change, 12(2), 147-158. http://doi.org/10.1007/s10833011-9154-1

Datnow, A., \& Castellano, M. (2000). Teachers' responses to success for all: How beliefs, experiences, and adaptations shape implementation. American Educational Research Journal, 37(3), 775799. http://doi.org/10.3102/00028312037003775

Datnow, A., \& Hubbard, L. (2015). Teachers' use of assessment data to inform instruction: Lessons from the past and prospects for the future. Teachers College Record, 117(4).

Datnow, A., \& Stringfield, S. (2011). Working together for reliable school reform. Journal of Education for Students Placed at Risk, 5(1-2), 183-204. http://doi.org/10.1080/10824669.2000.9671386

Datnow, A., Hubbard, L., \& Mehan, H. (1998). Educational reform implementation: A co-constructed process: Technical report. Santa Cruz, CA: Center for Research on Education, Diversity, and Excellence.

Datnow, A., Park, V., \& Wohlstetter, P. (2007). Achieving with data: How high-performing school systems use data to improve instruction for elementary students. San Fransisco: Center on Educational Governance University of California.

De Jong, R., \& Westerhof, K. J. (2001). The quality of student ratings of teacher behaviour. Learning Environments Research, 4(1), 51-85. http://doi.org/10.1023/A:1011402608575

Den Brok, P., Bergen, T., Stahl, R. J., \& Brekelmans, M. (2004). Students' perceptions of teacher control behaviours. Learning and Instruction, 14, 425-443. http://doi.org/10.1016/j. learninstruc.2004.01.004

Desimone, L. M. (2002). How can comprehensive school reform models be successfully implemented? Review of Educational Research, 72(3), 433-479. http://doi.org/10.3102/00346543072003433

Desimone, L. M. (2006). Consider the source: Response differences among teachers, principals and districts on survey questions about their education policy environment. Educational Policy, 20(4), 640-676. http://doi.org/10.1177/0895904805284056

Desimone, L. M. (2009). Improving impact studies of teachers ' professional development: Toward better conceptualizations and measures. Educational Researcher, 38(3), 181-199. 
Desimone, L. M., Porter, A. C., Garet, M. S., Yoon, K. S., \& Birman, B. F. (2002). Effects of professional development on teachers' instruction: Results from a three-year longitudinal study. Educational Evaluation and Policy Analysis, 24(2), 81-112. Retrieved from http://www.jstor.org/ stable/3594138

Devine, D. J., Clayton, L. D., Philips, J. L., Dunford, B. B., \& Melner, S. B. (1999). Teams in organizations: Prevalence, characteristics, and effectiveness. Small Group Research, 30, 678-711. http://doi. org/10.1177/104649649903000602

Dunn, K. E., Airola, D. T., Lo, W.-J., \& Garrison, M. (2013). Becoming data driven: The Influence of teachers' sense of efficacy on concerns related to data-driven decision making. The Journal of Experimental Education, 81(2), 222-241. http://doi.org/10.1080/00220973.2012.699899

Earl, L., \& Fullan, M. (2003). Using data in leadership for learning. Cambridge Journal of Education, 33(3), 383-394. http://doi.org/10.1080/0305764032000122023

Earl, L., \& Katz, S. (2006). Leading schools in a data-rich world: Harnessing data for school improvement. Thousans Oaks: Corwin Press.

Ertesvåg, S. K. (2009). Classroom leadership: the effect of a school development programme. Educational Psychology, 29(5), 515-539. http://doi.org/10.1080/01443410903122194

Faber, J. M., \& Visscher, A. J. (2014). Digitale leerlingvolgsystemen: een review van de effecten op leerprestaties [Digital student monitoring systems: A review of the effects on student performance]. Zoetermeer, The Netherlands. Retrieved from http://www.kennisnet.nl/ onderzoek/alle-onderzoeken/digitale-leerlingvolgsystemen-een-review-van-de-effecten-opleerprestaties/

Farley-Ripple, E., \& Buttram, J. (2015). The development of capacity for data use: The role of teacher networks in an elementary school. Teachers College Record, 117(4), 1-34.

Fauth, B., Decristan, J., Rieser, S., Klieme, E., \& Büttner, G. (2014). Student ratings of teaching quality in primary school: Dimensions and prediction of student outcomes. Learning and Instruction, 29, 1-9. http://doi.org/10.1016/j.learninstruc.2013.07.001

Ferguson, R. F. (2012). Can student surveys measure teaching quality? Phi Delta Kappan, 94, 24-28.

Ferron, J. M., Moeyaert, M., Van den Noortgate, W., \& Beretvas, S. N. (2014). Estimating causal effects from multiple-baseline studies: Implications for design and analysis. Psychological Methods, 19(4), 493-510. http://doi.org/10.1037/a0037038

Fink, D., \& Brayman, C. (2006). School leadership succession and the challenges of change. Educational Administration Quarterly, 42(1), 62-89. http://doi.org/10.1177/0013161X05278186

Finnigan, K. S., \& Daly, A. J. (2012). Mind the gap: Organizational learning and improvement in an underperforming urban system. American Journal of Education, 119(1), 41-71.

Fox, J.-P. (2010). Bayesian item response modeling. New York, NY: Springer.

Fox, J.-P., \& Glas, C. A. W. (2001). Bayesian estimation of a multilevel IRT model using Gibbs sampling. Psychometrika, 66(2), 271-288.

Fox, J., Friendly, M., \& Weisberg, S. (2013). Hypothesis tests for multivariate linear models using the Car package. The R Journal, 5, 39-52.

Fox, J.-P. (2007). Multilevel IRT modeling in practice with the package mlirt. Journal of Statistical Software, 20(5). http://doi.org/10.18637/jss.v020.i05

Frank, K. A., Penuel, W. R., \& Krause, A. (2015). What is a "good" social network for policy implementation? The flow of know-how for organizational change. Journal of Policy Analysis and Management, 34(2), 378-402. http://doi.org/10.1002/pam.21817

Fuchs, L. S., \& Fuchs, D. (1986). Effects of systematic formative evaluation: A meta-analysis. Exceptional Children, 53(199-208).

Gaertner, H. (2014). Effects of student feedback as a method of self-evaluating the quality of teaching. 
Studies in Educational Evaluation, 42, 91-99. http://doi.org/10.1016/j.stueduc.2014.04.003

Gage, N.A., \&Lewis, T.J. (2012). Hierarchicallinearmodelingmeta-analysisofsingle-subjectdesignresearch. The Journal of Special Education, 48(1), 3-16. http://doi.org/10.1177/0022466912443894

Garet, M. S., Porter, A. C., Desimone, L. M., Birman, B. F., \& Yoon, K. S. (2001). What makes professional development effective? Results from a national sample of teachers. American Educational Research Journal, 38(4), 915-945. Retrieved from http://www.jstor.org/stable/3202507

Gerzon, N. (2015). Structuring Professional Learning to Develop a Culture of Data Use: Aligning Knowledge From the Field and Research Findings. Teachers College Record, 117(4), 1-13.

Gitomer, D. H., \& Bell, C. A. (2013). Evaluating teaching and teachers. In K. F. Geisinger (Ed.), APA handbook of testing and assessment in psychology (pp. 415-444). Washington, DC: American Psychological Association.

Goe, L., Bell, C., \& Little, O. (2008). Approaches to evaluating teacher effectiveness: A research synthesis. Washington, DC: National Comprehensive Center for Teacher Quality. Retrieved from http://search.proquest.com.libproxy.library.wmich.edu/eric/ docview/881466794/13ED180BEAEE4BB92B/2?accountid=15099

Goldring, E., Huff, J., May, H., \& Camburn, E. (2008). School context and individual characteristics: what influences principal practice? Journal of Educational Administration, 46(3), 332-352.

Goldring, E., Mavrogordato, M., \& Haynes, K. T. (2014). Multi-Source principal evaluation data: how principals approach, interpret and use teacher feedback regarding their leadership effectiveness. Educational Administration Quarterly, (414), 1-28. http://doi.org/10.1177/0013161X14556152

Gorter, R., Fox, J.-P., \& Twisk, J. R. (2015). Why item response theory should be used for longitudinal questionnaire data analysis in medical research. BMC Medical Research Methodology, 15. http://doi.org/doi.org/10.1186/s12874-015-0050-x

Gotch, C. M., \& French, B. F. (2013). Elementary teachers' knowledge and self-efficacy for measurement concepts. The Teacher Educator, 48(1), 46-57. http://doi.org/10.1080/08878730.2012.740150

Graham, S., \& Santangelo, T. (2014). Does spelling instruction make students better spellers, readers, and writers? A meta-analytic review. Reading and Writing, 27(9), 1703-1743. http://doi. org/10.1007/s11145-014-9517-0

Graham, S., Morphy, P., Harris, K. R., Fink-Chorzempa, B., Saddler, B., Moran, S., \& Mason, L. (2008). Teaching spelling in the primary grades: A national survey of instructional practices and adaptations. American Educational Research Journal, 45(3), 796-825. http://doi. org/10.3102/0002831208319722

Gray, J. (2002). Jolts and reactions: Two decades of feeding back information on schools' performance. In A.J. Visscher, \& R. Coe (Eds). School improvement through performance feedback (pp. 143162). Lisse: Swets \& Zeitlinger B.V.

Guarino, C. M., Reckase, M. D., \& Wooldridge, J. M. (2015). Policy and research challenges of moving toward best practices in using student test scores to evaluate teacher performance. Journal of Research on Educational Effectiveness, 8(1), 1-7. http://doi.org/10.1080/19345747.2015.986 943

Hallgren, K. a. (2012). Computing inter-rater reliability for observational data: An overview and tutorial. Tutorials in Quantitative Methods for Psychology, 8(1), 23-34. http://doi.org/10.1016/j. biotechadv.2011.08.021.Secreted

Hallinger, P. (2010). A review of three decades of doctoral studies using the Principal Instructional Management Rating Scale: A lens on methodological progress in educational leadership. Educational Administration Quarterly, 271-306. http://doi.org/10.1177/0013161X10383412

Hallinger, P., \& Heck, R. H. (2011). Exploring the journey of school improvement: classifying and analyzing patterns of change in school improvement processes and learning outcomes. School 
Effectiveness and School Improvement, 22(1), 1-27. http://doi.org/10.1080/09243453.2010.5 36322

Hallinger, P., \& Murphy, J. (1985). Assessing the instructional management behavior of principals. The Elementary School Journal, 86(2), 217-248. http://doi.org/10.1086/461445

Hamilton, L., Halverson, R., Jackson, S. S., Mandinach, E. B., Supovitz, J. A., \& Wayman, J. C. (2009). Using student achievement data to support instructional decision making. Washington, DC. Retrieved from http://ies.ed.gov/ncee/wwc/pdf/practice_guides/dddm_pg_092909.pdf

Hammond, P., \& Yeshanew, T. (2007). The impact of feedback on school performance. Educational Studies, 33(2), 99-113. http://doi.org/10.1080/03055690601068212

Hamre, B. K., \& Pianta, R. C. (2006). Student-teacher relationships. In G. G. Bear \& M. K. M (Eds.), Children's needs III: Development, prevention and intervention (pp. 49-60). Washington, DC: National Association of School Psychologists.

Hanushek, E. A. (2011). How much is a good teacher worth? Education Next, 11(3), 40-45.

Hattie, J. (2009). Visible learning: A synthesis of over 800 meta-analyses relating to achievement. London: Routledge.

Hattie, J., \& Timperley, H. (2007). The power of feedback. Review of Educational Research, 77(1), 81112. http://doi.org/10.3102/003465430298487

Heck, R. H., \& Hallinger, P. (2009). Assessing the contribution of distributed leadership to school improvement and growth in math achievement. American Educational Research Journal, 46(3), 659-689. http://doi.org/10.3102/0002831209340042

Hedges, L. V. (2016). Challenges in building usable knowledge in education. Washington, DC: Keynote presented at Spring 2016 Conference SREE.

Henderson, S., Petrosiono, A., Guckenburg, S., \& Hamilton, S. (2007). Measuring how benchmark assessments af fect student achievement Measuring how benchmark assessments affect student achievement. (Issues \& Answers Report, REL 2007-No. 039). Washington, DC. Retrieved from http://ies.ed.gov/ncee/edlabs

Hill, H. C., Charalambous, C. Y., \& Kraft, M. a. (2012). When rater reliability is not enough: Teacher observation systems and a case for the generalizability study. Educational Researcher. http:// doi.org/10.3102/0013189X12437203

Hout, A. van den, Fox, J.-P., \& Klein Entink, R. (2015). Bayesian inference for an illness-death model for stroke with cognition as a latent time-dependent risk factor. Statistical Methods in Medical Research, 25(6), 769-787. http://doi.org/10.1177/0962280211426359

Huguet, A., Marsh, J. A., \& Farrell, C. C. (2014). Building teachers' data-use capacity: Insights from strong and developing coaches. Education Policy Analysis Archives, 22(52), 1-31.

Huizinga, T., Handelzalts, A., Nieveen, N., \& Voogt, J. (2015). Fostering teachers' design expertise in teacher design teams: conducive design and support activities. Curriculum Journal, 26(1), 137163. Retrieved from http://dx.doi.org/10.1080/09585176.2014.990395

Ikemoto, G. S., \& Marsh, J. A. (2007). Cutting through the "data-driven" mantra: Different conceptions of data-driven decision making. In Evidence and decision making: Yearbook of the national society of education (pp. 105-131). http://doi.org/10.1111/j.1744-7984.2007.00099.x

Ingram, D., Louis, K. S., \& Schroeder, R. G. (2004). Accountability policies and teacher decision making: Barriers to the use of data to improve practice. Teachers College Record, 106(6), 1258-1287.

Inspectie van het Onderwijs. (2010). Opbrengstgericht werken in het basisonderwijs [Data-based decision making in primary eduction]. Utrecht, The Netherlands.

Inspectie van het Onderwijs. (2011). De staat van het onderwijs: Onderwijsverslag 2010/2011 [The state of the educational system: the 2010/2011 Report on Education]. Utrecht, The Netherlands.

Inspectie van het Onderwijs. (2012). Beoordeling van opbrengsten in het basisonderwijs [Evaluation of 
student achievement in primary education]. Utrecht, The Netherlands.

Inspectie van het Onderwijs. (2013). De staat van het onderwijs: Onderwijsverslag 2011/2012 [The state of the educational system: the 2011/2012 Report on Education]. Utrecht, The Netherlands.

Inspectie van het Onderwijs. (2014). De staat van het onderwijs: Onderwijsverslag 2012/2013 [The state of the educational system: the 2012/2013 Report on Education]. Utrecht, The Netherlands.

Inspectie van het Onderwijs. (2015). De staat van het onderwijs: Onderwijsverslag 2013/2014 [The state of the educational system: the 2013/2014 Report on Education]. Utrecht, The Netherlands. Retrieved from https://mijn.govunited.nl/blg-221895.pdf

Ioannidis, J. P. A. (2005). Why most published research findings are false. PLoS Medicine, 2(8), e124. http://doi.org/10.1371/journal.pmed.0020124

Jackson, C. (2011). Multi-state models for panel data: The msm package for R. Journal of Statistical Software, 38(8), 1-28.

Jackson, C. (2014). Multi-state modelling with R: the msm package. Version 1.3.2. Cambridge, UK. Retrieved from ftp://218.193.60.3/software/rsoft/msm/doc/msm-manual.pdf

Jenson, W. R., Clark, E., Kircher, J. C., \& Kristjansson, S. D. (2007). Statistical reform: Evidence-based practice, meta-analyses, and single subject designs. Psychology in the Schools, 44(5), 483-493. http://doi.org/10.1002/pits.20240

Jimerson, B., \& Wayman, J. C. (2015). Professional learning for using data: Examining teacher needs and supports. Teachers College Record, 117(4), 1-36.

Kamphuis, F., \& Moelands, F. (2000). A student monitoring system. Educational Measurement: Issues and Practice, 28-30.

Kaufman, T., Graham, C., Picciano, A., Popham, J. A., \& Wiley, D. (2014). Data-driven decision making in the K-12 classroom. In J. M. Spector, M. D. Merrill, J. Elen, \& M. J. Bishop (Eds.), Handbook of research on educational communications and technology SE - 27 (pp. 337-346). Springer New York. http://doi.org/10.1007/978-1-4614-3185-5_27

Keuning, T., van Geel, M., Visscher, A. J., Fox, J.-P., \& Moolenaar, N. (in press). The Transformation of Schools' Social Networks During a Data-Based Decision Making Reform. Teachers College Record.

Kilduff, M., \& Tsai, W. (2003). Social networks and organizations. Thousand Oaks, CA: Sage.

Kingston, N., \& Nash, B. (2011). Formative assessment: A meta-analysis and a call for research. Educational Measurement: Issues and Practice, 30(4), 28-37. http://doi.org/10.1111/j.17453992.2011.00220.x

Kirkup, C., Sizmur, J., Sturman, L., \& Lewis, K. (2005). Schools' use of data in teaching and learning. Retrieved from https://www.european-agency.org/adminvoalrhfwgdjliticzabxujs/agencyprojects/assessment-resource-guide/documents/2008/11/schools_use_of_data.pdf

Kluger, A. N., \& DeNisi, A. (1996). The effects of feedback interventions on performance: A historical review, a meta-analysis, and a preliminary feedback intervention theory. Psychological Bulletin, 199(2), 254-284.

Koh, K. H. (2011). Improving teachers' assessment literacy through professional development. Teaching Education, 22(3), 255-276. http://doi.org/10.1080/10476210.2011.593164

Konstantopoulos, S., Miller, S. R., \& van der Ploeg, a. (2013). The impact of Indiana's system of interim assessments on mathematics and reading achievement. Educational Evaluation and Policy Analysis, 35(4), 481-499. http://doi.org/10.3102/0162373713498930

Koth, C. W., Bradshaw, C. P., \& Leaf, P. J. (2008). A multilevel study of predictors of student perceptions of school climate: The effect of classroom-level factors. Journal of Educational Psychology, 100(1), 96-104. http://doi.org/10.1037/0022-0663.100.1.96

Kratochwill, T. R., Hitchcock, J., Horner, R. H., Levin, J. R., Odom, S. L., Rindskopf, D. M., \& Shadish, W. 
R. (2010). Single-case design technical documentation. Retrieved from http://ies.ed.gov/ncee/ wwc/pdf/wwc_scd.pdf

Kyriakides, L. (2005). Drawing from teacher effectiveness research and research into teacher interpersonal behaviour to establish a teacher evaluation system: A study on the use of student ratings to evaluate teacher behaviour. Journal of Classroom Interaction, 40(2), 44-66.

Lai, K. M., Mcnaughton, S., Amituanai-toloa, M., Turner, R., \& Hsiao, S. (2009). Sustained acceleration of achievement in reading comprehension: The New Zealand experience. Reading Research Quarterly, 44(1), 30-56.

Lai, M. K., \& McNaughton, S. (2009). Raising student achievement in poor communities through evidencebased conversations. In L. Earl \& H. Timperley (Eds.), Professional Learning Converstations: CHallenges in Using Evidence for Improvement (pp. 13-27). Springer.

Lai, M. K., \& McNaughton, S. (2013). Analysis and discussion of classroom and achievement data to raise student achievement. In K. Schildkamp, M. K. Lai, \& L. Earl (Eds.), Data-based decision making in education: Challenges and opportunities (pp. 23-48). Dordrecht: Springer. http://doi. org/10.1007/978-94-007-4816-3

Lai, M. K., \& Schildkamp, K. (2013). Data-based decision making: An overview. In K. Schildkamp, M. K. Lai, \& L. Earl (Eds.), Data-based decision making in education: Challenges and opportunities (pp. 9-21). Dordrecht: Springer Netherlands. http://doi.org/10.1007/978-94-007-4816-3

Lam, A. C., Ruzek, E. A., Schenke, K., Conley, A. M., \& Karabenick, S. A. (2015). Student perceptions of classroom achievement goal structure: Is it appropriate to aggregate? Journal of Edaucational Psychology, 107(4), 1102-1115. http://doi.org/10.1037/edu0000028

Leithwood, K., Harris, A., \& Hopkins, D. (2008). School leadership \& management: Seven strong claims about successful school leadership. School Leadership \& Management, 28(1), 27-42. http://doi. org/10.1080/13632430701800060

Leithwood, K., Patten, S., \& Jantzi, D. (2010). Testing a conception of how school leadership influences student learning. Educational Administration Quarterly, 46, 671-706. http://doi. org/10.1177/0013161X10377347

Levin, J. A., \& Datnow, A. (2012). The principal role in data-driven decision making: Using case-study data to develop multi-mediator models of educational reform. School Effectiveness and School Improvement, 23(2), 179-201. http://doi.org/10.1080/09243453.2011.599394

Little, A. W. (2001). Multigrade teaching: Towards an international research and policy agenda. International Journal of Educational Development, 21, 481-497. http://doi.org/10.1016/S07380593(01)00011-6

Little, A. W. (2006). Education for all and multigrade teaching: Challenges and opportunities. Education for All and Multigrade Teaching. Dordrecht, The Netherlands: Springer. http://doi. org/10.1007/1-4020-4591-3

Liu, K., Springer, J., Stuit, D., \& Wan, Y. (2014). The utility of teacher and student surveys in principal evaluations: An empirical investigation. (REL 2015-047). Washington, DC.

Locke, E. a., \& Latham, G. P. (2002). Building a practically useful theory of goal setting and task motivation: A 35-year odyssey. American Psychologist, 57(9), 705-717. http://doi.org/10.1037//0003066X.57.9.705

Love, N., Stiles, K. E., Mundry, S., \& DiRanna, K. (2008). A data coach's guide to improve learning for all students: Unleashing the power of collaborative inquiry. Thousand Oaks, CA: Corwin Press.

Lukociene, O., Varriale, R., \& Vermunt, J. K. (2010). The simultaneous decision(s) about the number of lower- and higher-level classes in multilevel latent class analysis. Social Methodology, 1(40), 247-283.

Lumpe, A. T. (2007). Research-based professional development: Teachers engaged in professional 
learning communities. Journal of Science Teacher Education, 18(1), 125-128. http://doi. org/10.1007/s10972-006-9018-3

Makel, M. C., \& Plucker, J. A. (2014). Facts are more important than novelty: Replication in the education sciences. Educational Researcher, 43(6), 304-316. http://doi.org/10.3102/0013189X14545513

Mandinach, E. B. (2012). A perfect time for data use: Using data-driven decision making to inform practice. Educational Psychologist, 47(2), 71-85. http://doi.org/10.1080/00461520.2012.667 064

Mandinach, E. B., \& Gummer, E. S. (2013a). A systemic view of implementing data literacy in educator preparation. Educational Researcher, 42(1), 30-37. http://doi.org/10.3102/0013189X12459803

Mandinach, E. B., \& Gummer, E. S. (2013b). Defining data literacy: A report on a convening of experts. The Journal of Educational Research \& Policy Studies, 13(2), 6-28.

Mandinach, E. B., \& Gummer, E. S. (2015). Data driven decision making: Components of the enculturation of data use in education. Teachers College Record, 117(4), 1-5. Retrieved from http://www. tcrecord.org

Mandinach, E. B., Gummer, E. S., \& Muller, R. D. (2011). The complexities of integrating data-driven decision making into professional preparation in schools of education: It 's harder than you think. Report from an invitational meeting. Alexandria, VA, Portland, OR, and Washington, DC.

Mandinach, E. B., Honey, M., \& Light, D. (2006). A theoretical framework for data-driven decision making. Paper presented at the annual meeting of AERA, San Francisco (pp. 1-18).

Marsh, J. A. (2012). Interventions promoting educators use of data: Research insights and gaps. Teachers College Record, 114(11), 1-47.

Marsh, J. A., \& Farrell, C. C. (2014). How leaders can support teachers with data-driven decision making: A framework for understanding capacity building. Educational Management Administration \& Leadership, 43(2), 269-289. http://doi.org/10.1177/1741143214537229

Marsh, J. A., Bertrand, M., \& Huguet, A. (2015). Using data to alter instructional practice: The mediating role of coaches and professional learning communities. Teachers College Record, 117(4), 1-40.

Marsh, J. A., Pane, J. F., \& Hamilton, L. S. (2006). Making sense of data-driven decision making in education. Santa Monica, CA.

Martin, M. O., Mullis, I. V. S., \& Foy, P. (2008). TIMSS 2007 International mathematics report: Findings from IEA's trend in international mathematics and science study at the fourth and eighth grades. Chestnut Hill, MA.

May, H., \& Robinson, M. A. (2007). A randomized evaluation of Ohio's personalized assessment reporting system (PARS). Madison, WI.

McCaffrey, D. F., \& Hamilton, L. S. (2007). Value-added assessment in practise: Lessons from the Pennsy/vania value-added assessment system pilot project. Santa Monica, CA: RAND Corporation. Retrieved from http://www.rand.org/pubs/technical_reports/TR506

Means, B., Padilla, C., \& Gallagher, L. (2010). Use of education data at the local level: From accountability to instructional improvement. Retrieved from http://www2.ed.gov/rschstat/eval/tech/use-ofeducation-data/use-of-education-data.pdf

Moolenaar, N. M. (2010). Ties with potential: Nature, antecedents, and consequences of social networks in school teams. University of Amsterdam.

Moolenaar, N. M. (2012). A social network perspective on teacher collaboration in schools: Theory, methodology, and applications. American Journal of Education, 119(1), 7-39.

Moolenaar, N. M., Daly, A. J., \& Sleegers, P. J. C. (2010). Occupying the principal position: Examining relationships between transformational leadership, social network position, and schools' innovative climate. Educational Administration Quarterly, 46(5), 623-670. http://doi. org/10.1177/0013161X10378689 
Moolenaar, N. M., Daly, A. J., \& Sleegers, P. J. C. (2011). Ties with potential: Social network structure and innovative climate in Dutch schools. Teachers College Record, 113(9), 1983-2017.

Moolenaar, N. M., Daly, A. J., Cornelissen, F., Liou, Y. H., Caillier, S., Riordan, R., Wilson, K., \& Cohen, N. A. (2014). Linked to innovation: Shaping an innovative climate through network intentionality and educators' social network position. Journal of Educational Change, 15, 99-123. http://doi. org/10.1007/s10833-014-9230-4

Moolenaar, N. M., Sleegers, P. J. C., Karsten, S., \& Daly, A. J. (2012). The social fabric of elementary schools: A network typology of social interaction among teachers. Educational Studies, 38(4), 355-371. http://doi.org/10.1080/03055698.2011.643101

Morisano, D., \& Locke, E. A. (2013). Goal setting and academic achievement. In J. Hattie \& E. M. Anderman (Eds.), International guide to student achievement (pp. 45-48). New York and London: Routledge.

Mullis, I. V. S., Martin, M. O., Foy, P., \& Arora, A. (2012). TIMSS 2011 international results in mathematics. Chestnut Hill, MA.

Mullis, I. V. S., Martin, M. O., Kennedy, A. M., \& Foy, P. (2007). PIRLS 2006 International report: IEA's progress in international reading literacy study in primary schools in 40 countries. Chestnut Hill, MA: TIMSS \& PIRLS International Study Center, Boston College.

Muthén, L. K., \& Muthén, B. O. (2004). Mplus user's Guide. Los Angeles, California.

Nye, B., Konstantopoulos, S., \& Hedges, L. V. (2004). How large are teacher effects? Educational Evaluation and Policy Analysis, 26(3), 237-257.

OECD. (2007). PISA 2006: Science competencies for tomorrow's world: Volume 1: Analysis. Paris.

Oláh, L. N., Lawrence, N. R., \& Riggan, M. (2010). Learning to learn from benchmark assessment data: How teachers analyze results. Peabody Journal of Education, 85(2), 226-245. http://doi. org/10.1080/01619561003688688

Orland, M. (2015). Research and policy perspectives on data based decision making in education. Teachers College Record, 117(4).

Park, V., Daly, A. J., \& Guerra, A. W. (2012). Strategic framing: How leaders craft the meaning of data use for equity and learning. Educational Policy, 27(4), 645-675. http://doi. org/10.1177/0895904811429295

Penuel, W. R., \& Riel, M. (2007). The "new" science of networks and the challenge of school change. Phi Delta Kappan, 88, 611-615. http://doi.org/10.3102/0002831207308221

Penuel, W. R., Sun, M., Frank, K. A., \& Gallagher, H. A. (2012). Using social network analysis to study how collegial interactions can augment teacher learning from external professional development. American Journal of Education, 119(1), 103-136.

Peterson, K. D., Wahlquist, C., \& Bone, K. (2000). Student Surveys for school teacher evaluation. Journal of Personnel Evaluation in Education, 14(2), 135-153.

Pianta, R. C., Hamre, B. K., Haynes, N. J., Mintz, S., \& La Paro, K. M. (2007). Classroom assessment scoring system manual, middel/secondary version. Charlottesville: University of Virginia.

Popham, W. (2011). Assessment literacy overlooked: A teacher educator's confession. The Teacher Educator, 46(4), 265-273. http://doi.org/10.1080/08878730.2011.605048

PO-Raad. (2010). Eenpitters: Praktijkervaringen en oplossingen voor knelpunten [One school school boards: Practical experiences and solutions for bottlenecks]. Utrecht, The Netherlands.

Porter, A. C., Polikoff, M. S., Goldring, E., Murphy, J., Elliott, S. N., \& May, H. (2010). Developing a psychometrically sound assessment of school leadership: The VAL-ED as a case study. Educational Administration Quarterly, 46(2), 135-173. http://doi.org/10.1177/1094670510361747

Quint, J. C., Sepanik, S., \& Smith, J. K. (2008). using student data to improve teaching and learning: findings from an evaluation of the Formative Assessments of Student Thinking in Reading 
(FAST-R) Program in Boston elementary schools. New York, NY: MDRC.

R Core Team. (2014). R: A language and environment for statistical computing. Vienna, Austria: R Foundation for Statistical Computing. Retrieved from http://www.r-project.org

R CoreTeam. (2013). R: A language and environment for statistical computing. Vienna, Austria: R Foundation for Statistical Computing. Retrieved from http://www.r-project.org/

Raudenbush, S. W., \& Bryk, A. S. (2002). Hierarchical linear models: Applications and data analysis methods. Advanced quantitative techniques in the social sciences 1 (Vol. 2nd). Sage Publications.

Reeves, P. L., \& Burt, W. L. (2006). Challenges in data-based decision-making: Voices from principals. Educational Horizons, 65-71.

Reise, S. P., Widaman, K. F., \& Pugh, R. H. (1993). Confirmatory factor analysis and item response theory: Two approaches for exploring measurement invariance. Psychological Bulletin, 114, 552-566. http://doi.org/10.1037/0033-2909.114.3.552

Ritzema, E. S. (2015). Professional development in data use: The effects of primary school teacher training on teaching practices and students' mathematical proficiency. University of Groningen.

Robinson, V. M. J., Lloyd, C. a., \& Rowe, K. J. (2008). The Impact of leadership on student outcomes: An analysis of the differential effects of leadership types. Educational Administration Quarterly, 44, 635-674. http://doi.org/10.1177/0013161X08321509

Samejima, F. (1997). Graded response model. In W. van der Linden \& R. K. Hambleton (Eds.), Handbook of modern item response theory (pp. 85-100). New York, NY: Springer.

Scheerens, J., \& Bosker, R. J. (1997). The foundations of educational effectiveness. Oxford: Elsevier Ltd.

Schildkamp, K., \& Ehren, M. (2013). From "intuition"- to "data"-based decision making in Dutch secondary schools? In K. Schildkamp, M. K. Lai, \& L. Earl (Eds.), Data-based decision making in education: Challenges and opportunities (pp. 49-68). Dordrecht: Springer. http://doi. org/10.1007/978-94-007-4816-3

Schildkamp, K., \& Kuiper, W. (2010). Data-informed curriculum reform: Which data, what purposes, and promoting and hindering factors. Teaching and Teacher Education, 26(3), 482-496. http://doi. org/10.1016/j.tate.2009.06.007

Schildkamp, K., \& Lai, M. K. (2013). Conclusion and a data use framework. In K. Schildkamp, M. K. Lai, \& L. Earl (Eds.), Data-based decision making in Education: Challenges and opportunities (pp. 177-191). Dordrecht: Springer. http://doi.org/10.1007/978-94-007-4816-3

Schildkamp, K., \& Lai, M. K. (2013b). Introduction. In K. Schildkamp, M. K. Lai, \& L. Earl (Eds.), Data-based decision making in education: Challenges and opportunities (pp. 1-7). Dordrecht: Springer. http://doi.org/10.1007/978-94-007-4816-3

Schildkamp, K., \& Poortman, C. L. (2015). Factors influencing the function of data teams. Teachers College Record, 117(4). Retrieved from http://www.tcrecord.org/content.asp?contentid=17851

Schildkamp, K., Ehren, M., \& Lai, M. K. (2012). Editorial article for the special issue on data-based decision making around the world: from policy to practice to results. School Effectiveness and School Improvement, 23(2), 123-131. http://doi.org/10.1080/09243453.2011.652122

Schildkamp, K., Karbautzki, L., \& Vanhoof, J. (2014). Exploring data use practices around Europe: Identifying enablers and barriers. Studies in Educational Evaluation, 42, 15-24. http://doi. org/10.1016/j.stueduc.2013.10.007

Schildkamp, K., Lai, M. K., \& Earl, L. (2013). Data-based Decision making in education: Challenges and opportunities. (K. Schildkamp, M. K. Lai, \& L. Earl, Eds.). Dordrecht: Springer Netherlands. http:// doi.org/10.1007/978-94-007-4816-3

Schildkamp, K., Poortman, C. L., \& Handelzalts, A. (2015). Data teams for schoolimprovement. School Effectiveness and School Improvement: An International Journal of Research, Policy and Practice. http://doi.org/10.1080/09243453.2015.1056192 
Schmidt, S. (2009). Shall we really do it again? The powerful concept of replication is neglected in the social sciences. Review of General Psychology, 13(2), 90-100. http://doi.org/10.1037/a0015108

Scott, J. (2013). Social network analysis (third). London, UK: SAGE Publication Ltd.

Seidel, T., \& Shavelson, R. J. (2007). Teaching effectiveness research in the past decade: The role of theory and research design in disentangling meta-analysis results. Review of Educational Research, 77(4), 454-499. http://doi.org/10.3102/0034654307310317

Shadish, W. R. (2014). Analysis and meta-analysis of single-case designs: An introduction. Journal of School Psychology, 52(2), 109-122. http://doi.org/http://dx.doi.org/10.1016/j.jsp.2013.11.009

Shadish, W. R., \& Sullivan, K. J. (2011). Characteristics of single-case designs used to assess intervention effects in 2008. Behavior Research Methods, 43(4), 971-980. http://doi.org/10.3758/s13428011-0111-y

Shadish, W. R., Cook, T. D., \& Campbell, D. T. (2002). Experimental and quasi-experimental designs for generalized causal inference. Bostan, MA: Houghton Mifflin.

Shadish, W. R., Kyse, E. N., \& Rindskopf, D. M. (2013). Analyzing data from single-case designs using multilevel models: New applications and some agenda items for future research. Psychological Methods. 18(3), 385. http://doi.org/10.1037/a0032964

Sklar, J. C., \& Zwick, R. (2009). Multimedia presentations in educational measurement and statistics: Design considerations and instructional approaches. Journal of Statistics Education, 17(1991), 1-14. Retrieved from http://www.amstat.org/publications/jse/v17n3/sklar.pdf

Slavin, R. E., Cheung, A., Holmes, G., Madden, N. A., \& Chamberlain, A. (2012). Effects of a data-driven district reform model on state assessment outcomes. American Educational Research Journal, 50(2), 371-396. http://doi.org/10.3102/0002831212466909

Snijders, T. A. B. (2001). The statistical evaluation of social network dynamics. In M. E. Sobel \& M. P. Becker (Eds.), Sociological Methodology (pp. 361-395). Boston and London: Basil Blackwell.

Snijders, T. A. B. (2005). Models for longitudinal network data. In P. Carrington, J. Scott, \& S. Wasserman (Eds.), Models and methods in social network analysis. New York: Cambridge University Press.

Snijders, T. A. B., \& Bosker, R. J. (1999). Multilevel analysis: An introduction to basic and advanced multilevel modeling. London, UK: Sage Publishers.

Snijders, T. A. B., Steglich, C. E. G., Schweinberger, M., \& Huisman, M. (2007). Manuel for SIENA version 3. Groningen, Oxford: University of Groningen, ICS, University of Oxford: Department of Statistics. Retrieved from http://stat.gamma.rug.nl/stocnet

Sparrowe, R. T., Liden, R. C., Wayne, S. J., \& Kraimer, M. L. (2001). Social networks and the performance of individuals and groups. Academy of Management Journal, 44(2), 316-325. http://doi. $\operatorname{org} / 10.2307 / 3069458$

Spillane, J. P., Kim, C. M., \& Frank, K. A. (2012). Instructional advice and information providing and receiving behavior in elementary schools: Exploring tie formation as a building block in social capital development. American Educational Research Journal, 49(6), 1112-1145. http://doi. org/10.3102/0002831212459339

Spooren, P., Brockx, B., \& Mortelmans, D. (2013). On the validity of student evaluation of teaching: The state of the art. Review of Educational Research, 83, 598-642. http://doi. org/10.3102/0034654313496870

Staman, L., Visscher, A. J., \& Luyten, H. (2013). The effects of training school staff for utilizing student monitoring system data. In D. Passey, A. Breiter, \& A. Visscher (Eds.), 10th IFIP WG 3.7 Conference, ITEM 2012 (pp. 3-14). Bremen, Germany: Springer.

Staman, L., Visscher, A. J., \& Luyten, H. (2014). The effects of professional development on the attitudes, knowledge and skills for data-driven decision making. Studies in Educational Evaluation, 42, 79-90. http://doi.org/10.1016/j.stueduc.2013.11.002 
Stone, C. A., \& Zhu, X. (2015). Bayesian analysis of item response theory models using SAS. Cary, NC: SAS Publications.

Sullivan, K. J., Shadish, W. R., \& Steiner, P. M. (2015). An introduction to modeling longitudinal data with generalized additive models: Applications to single-case designs. Psychological Methods, 20 (1), 26. http://doi.org/10.1037/met0000020

Sun, M., Penuel, W. R., Frank, K. A., Gallagher, H. A., \& Youngs, P. (2013). Shaping professional development to promote the diffusion of instructional expertise among teachers. Educational Evaluation and Policy Analysis, 35, 344-369. http://doi.org/10.3102/0162373713482763

Sung, Y.-T., Chang, K.-E., \& Liu, T.-C. (2016). The effects of integrating mobile devices with teaching and learning on students' learning performance: A meta-analysis and research synthesis. Computers \& Education, 94, 252-275. http://doi.org/10.1016/j.compedu.2015.11.008

Supovitz, J. A. (2012). Getting at student understanding- The key to teachers' use of test data. Teachers College Record, 114(11), 1-29.

Ten Bruggencate, G. C. (2009). Maken schoolleiders het verschil? Onderzoek naar de invloed van schoolleiders op de schoolresultaten [Do school leaders make a difference with respect to school performance?]. University of Twente.

Timperley, H. (2008). Teacher professional learning and development. International Academy of Education.

Tsai, W. (2001). Knowledge transfer in intraorganizational networks: Effects of network position and absorptive capacity on business unit innovation and performance. Academy of Management Journal, 44(5), 996-1004. http://doi.org/10.2307/3069443

Turner, E. O., \& Coburn, C. E. (2012). Interventions to promote data use: An introduction. Teachers College Record, 114(11), 1-13.

Urick, A., \& Bowers, A. J. (2014). What are the different types of principals across the United States? A latent class analysis of principal perception of leadership. Educational Administration Quarterly, 50(1), 96-134. http://doi.org/10.1177/0013161X13489019

Vaerenbergh, Y. Van, \& Thomas, T. (2013). Response styles in survey research: A literature review of antecedents, consequences, and remedies. International Journal of Public Opinion Research, 25(2), 195-217. Retrieved from http://ijpor.oxfordjournals.org/content/25/2/195.short

Van de Grift, W. J. C. M. (2014). Measuring teaching quality in several European countries. School Effectiveness and School Improvement, 25(3), 295-311. http://doi.org/10.1080/09243453.20 13.794845

Van de Grift, W. J. C. M., van der Wal, M., \& Torenbeek, M. (2011). Ontwikkeling in de pedagogisch didactische vaardigheid van leraren in het basisonderwijs [The development in primary school teachers' pedagogical skills]. Pedagogische Studiën, 88, 416-432.

Van den Noortgate, W., \& Onghena, P. (2003). Combining single-case experimental data using hierarchical linear models. School Psychology Quarterly, 18, 325-346.

Van den Noortgate, W., \& Onghena, P. (2003). Multilevel meta-analysis: A comparison with traditional meta-analytical procedures. Educational and Psychological Measurement, 63, 765-790. http:// doi.org/10.1177/0013164403251027

Van der Kleij, F. M., Vermeulen, J. A., Schildkamp, K., \& Eggen, T. J. H. M. (2015). Integrating databased decision making, assessment for learning and diagnostic testing in formative assessment. Assessment in Education: Principles, Policy \& Practice, 22(3), 324-343. http://doi.org/10.1080/ 0969594X.2014.999024

Van der Scheer, E., \& Visscher, A. J. (2014). The effects of an intensive one-year data-based decision making intervention for grade 4 teachers on students' mathematicsal achievement. Southampton (UK): Paper presentation at the EARLI SIG Educational Effectiveness. 
Van Geel, M., Keuning, T., Visscher, A. J., \& Fox, J.-P. (2016). Assessing the Effects of a SchoolWide Data-Based Decision-Making Intervention on Student Achievement Growth in Primary Schools. American Educational Research Journal, 53(2), 360-394. http://doi. org/10.3102/0002831216637346

Van Geel, M., Teunis, B., \& Visscher, A. J. (submitted). School characteristics that influence the implementation of a data-based decision making intervention.

Van Veen, K., Zwart, R., \& Meirink, J. (2011). What makes teacher professional development effective? A literature review. In M. Kooy \& K. Van Veen (Eds.), Teacher learning that matters (pp. 3-21). New York: Routledge.

Vanhoof, J., Van Petegem, P., \& De Maeyer, S. (2009). Attitudes towards school self-evaluation. Studies in Educational Evaluation, 35(1), 21-28. http://doi.org/10.1016/j.stueduc.2009.01.004

Vanhoof, J., Verhaeghe, G., Van Petegem, P., \& Valcke, M. (2013). Improving data literacy in schools: Lessons from the school feedback project. In K. Schildkamp, M. K. Lai, \& L. Earl (Eds.), Databased decision making in education: Challenges and opportunities (pp. 113-134). Dordrecht: Springer. http://doi.org/10.1007/978-94-007-4816-3

Veenman, S. (1996). Effects of multigrade and multi-age classes reconsidered. Review of Educational Research, 66(3), 323-340. http://doi.org/10.3102/00346543066003323

Verhaeghe, G. (2011). Characteristics of school performance feedback systems. In School performance feedback systems: Design and implementation issues (pp. 24-54), Doctoral dissertation, Ghent University).

Verhaeghe, G., Schildkamp, K., Luyten, H., \& Valcke, M. (2015). Diversity in school performance feedback systems. School Effectiveness and School Improvement, 26(4), 612-638. http://doi.org/10.108 0/09243453.2015.1017506

Vermunt, J. K. (2003). Multilevel latent class models. Sociological Methodology, 33(Lc), 213-239. http:// doi.org/10.1111/j.0081-1750.2003.t01-1-00131.x

Visscher, A. J. (2015). Over de zin van opbrengstgericht(er) werken in het onderwijs [On the value of data-based decision making in education]. Groningen: University of Groningen.

Visscher, A. J., \& Ehren, M. (2011). De eenvoud en complexiteit van Opbrengstgericht Werken. [The simplicity and complexity of data-driven teaching], 1-37. Retrieved from http://www. rijksoverheid.nl/documenten-en-publicaties/rapporten/2011/07/13/de-eenvoud-encomplexiteit-van-opbrengstgericht-werken.html

Wayman, J. C., \& Cho, V. (2008). Preparing educators to effectively use student data systems. In T. J. Kowalski \& T. J. Lasley (Eds.), Handbook on data-based decision-making in education (pp. 89104). New York: Routledge.

Wayman, J. C., Cho, V., Jimerson, J. B., \& Spikes, D. D. (2012). District-wide effects on data use in the classroom. Educational Policy Analysis Archives, 20(25).

Wayman, J. C., Midgley, S., \& Stringfield, S. (2006). Leadership for data-based decision making: collaborative educator teams. In A. Danzig, K. Borman, B. Jones, \& B. Wright (Eds.), New models of professional development for learner centered leadership (pp. 189-206). Mahwah, NJ: Erlbaum.

Wayman, J. C., Stringfield, S., \& Yakimowski, M. (2004). Software enabling school improvement through analysis of student data.

Zwick, R., Sklar, J. C., Wakefield, G., Hamilton, C., Norman, A., \& Folsom, D. (2008). Instructional Tools in Educational Measurement and Statistics (ITEMS) for school personnel: Evaluation of three webbased training modules. Educational Measurement: Issues and Practice, 27(2), 14-27. http:// doi.org/10.1111/j.1745-3992.2008.00119.x 
References 


\section{Samenvatting}

Het gebruik van data bij het maken van beslissingen is tegenwoordig in allerlei sectoren heel gangbaar. Van commerciële bedrijven die op basis van consumentendata hun verkoopstrategie aanpassen, tot ziekenhuizen die de effectiviteit van hun behandelingen evalueren. Ook in het onderwijs zien we deze trend: het is steeds beter mogelijk om op efficiënte wijze de prestaties en vorderingen van leerlingen te analyseren, waardoor leerkrachten hun onderwijs kunnen aanpassen op basis van de vastgestelde onderwijsbehoeften van hun leerlingen.

De aandacht voor datagebruik in het onderwijs heeft twee kanten, enerzijds wordt van scholen verwacht dat zij zich kunnen verantwoorden over de resultaten die ze met hun leerlingen behalen. Anderzijds gebruiken scholen data om hun onderwijs te verbeteren en het beter af te stemmen op de behoeften van hun leerlingen. Dit is natuurlijk niets nieuws: leerkrachten gebruiken altijd informatie over hun leerlingen. Zo stellen ze vragen, observeren ze leerlingen, en kijken ze gemaakt werk na. Dit gebeurt echter niet altijd systematisch. Het gebruik van onder andere digitale leerlingvolgsystemen stelt leerkrachten en scholen in staat om op meer systematische wijze gebruik te maken van gegevens, door deze in overzichtelijke grafieken en tabellen weer te geven en bijvoorbeeld ook door prestaties van leerlingen en groepen door de jaren heen te vergelijken. De uitdaging ligt erin deze data systematisch te benutten voor het bepalen van het onderwijsaanbod.

Het systematisch verzamelen en analyseren van data, met als doel het nemen van beslissingen om het onderwijs te verbeteren, staat internationaal bekend als 'databased decision making', oftewel DBDM. In de Nederlandse context wordt vaak de term 'opbrengstgericht werken' (OGW) gebruikt.

\section{Aanleiding}

De Nederlandse prestaties in internationale onderzoeken naar de schoolprestaties, vielen in 2007 tegen: Nederland was een van de weinige landen waar de prestaties in de kernvakken (rekenen en lezen) achteruit waren gegaan. OGW werd daarom speerpunt in het onderwijsbeleid. De Onderwijsinspectie toonde aan dat de prestaties hoger waren op scholen waar volgens de principes van OGW werd gewerkt (deze indicatoren zijn: de school gebruikt een samenhangend systeem van genormeerde instrumenten en procedures voor het volgen van de prestaties en de ontwikkeling van de leerlingen; de leraren volgen en analyseren systematisch de voortgang in de ontwikkeling van de leerlingen; de school evalueert regelmatig de effecten van de zorg; de school evalueert jaarlijks de resultaten van de leerlingen; de school evalueert regelmatig het onderwijsleerproces).

Het Ministerie van Onderwijs heeft de ambitie dat in 2018 maar liefst 90 procent van de Nederlandse basisscholen een voldoende scoort op alle vijf OGW-indicatoren van de Onderwijsinspectie. In de periode 2011-2014 schommelde dit rond de dertig procent. Om dit doel te bereiken stimuleert de overheid initiatieven die erop zijn gericht om OGW in scholen te implementeren en verankeren. Eén van deze initiatieven is het Focus-project, ontwikkeld door de Universiteit Twente. In dit proefschrift evalueren we de Focus-interventie, waarbij we ons enerzijds richten op de effecten op leerlingprestaties, en anderzijds op het bepalen van kenmerken van scholen en leerkrachten die samenhangen met de effectieve implementatie van OGW in de deelnemende scholen. 


\section{Het Focus-project}

In het Focus-project wordt de volgende definitie van OGW gehanteerd: het systematisch en doelgericht werken aan het maximaliseren van prestaties. Deze werkwijze wordt toegepast op groeps- en schoolniveau, en idealiter ook op het niveau van het schoolbestuur. In Figuur 1 zijn de componenten van OGW weergegeven: het analyseren en evalueren van resultaten; het stellen van SMART en uitdagende doelen; het bepalen van een strategie om deze doelen te bereiken; en het uitvoeren van deze strategie.

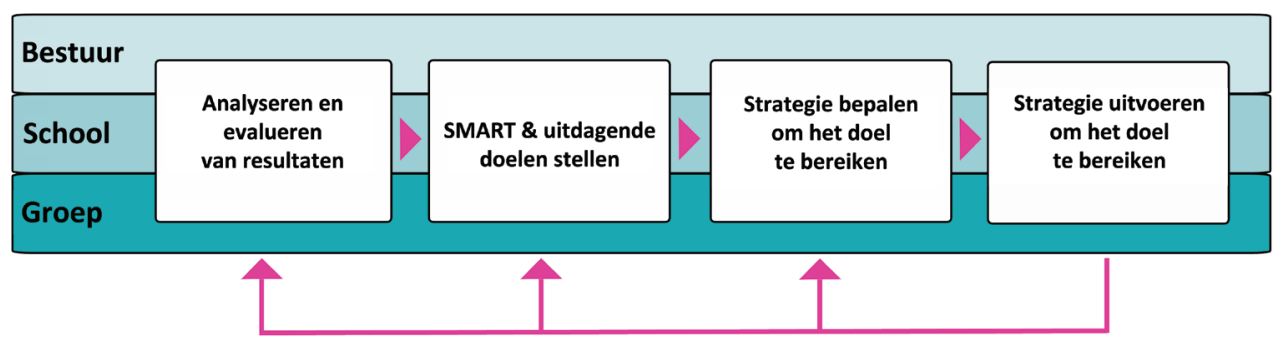

Figuur 1. Schematische weergave van OGW

De Focus-interventie was erop gericht OGW in de scholen te implementeren en verankeren, door de werkwijze zoals weergegeven in Figuur 1 te volgen. De training duurde twee opeenvolgende schooljaren, en werd door het gehele schoolteam van de deelnemende scholen gevolgd. Daarnaast was er op gezette tijden overleg met de schoolleider (en indien van toepassing het bestuur) om de voortgang te bespreken en bevorderen.

De eerste bijeenkomsten waren gericht op het verwerven van de kennis en vaardigheden die noodzakelijk worden geacht voor het implementeren van OGW: het analyseren van de gegevens in het leerlingvolgsysteem (LVS), het diagnosticeren van de onderwijsbehoeften van leerlingen, het stellen van doelen en het bepalen van een strategie voor de realisatie van de gestelde doelen (in de vorm van een groepsplan). Dit plan diende vervolgens te worden uitgevoerd in de school- en klaspraktijk, waarna de resultaten van het uitgevoerde plan werden geëvalueerd na de volgende afname van de toetsen uit het leerlingvolgsysteem. Deze evaluatie vond plaats op groepsniveau, maar ook op schoolniveau, het laatste in de vorm van een opbrengstenvergadering. De evaluatie vormde het startpunt van een nieuwe periode, met nieuwe doelen en een bijbehorende aanpak. Deze cyclus werd per schooljaar tweemaal doorlopen, met tussenevaluaties op basis van onder andere methodegebonden toetsen, dagelijks werk van leerlingen, en leerlingobservaties.

$\mathrm{Na}$ het eerste schooljaar konden scholen kiezen voor het verdiepen van OGW voor het vakgebied waarmee zij het eerste jaar bezig waren geweest, of voor het verbreden van OGW naar een ander vak. Daarnaast vonden in het tweede interventiejaar lesobservaties plaats, en was er aandacht voor collegiale consultatie, waarbij teamleden elkaar observeren om van elkaar te leren.

In de teambijeenkomsten lag de nadruk op het gebruik van de gegevens en inzichten om het onderwijs (nog) beter te laten aansluiten bij de behoeften van de leerlingen. De interventie was nadrukkelijk niet gericht op het verantwoordingsaspect dat ook wel wordt geassocieerd met OGW. 
In dit proefschrift zijn de effecten van twee versies van het Focus-project geëvalueerd: Focus II in de schooljaren 2011-2012-2013, en Focus III in de schooljaren 2012-2013-2014. Het grootste verschil tussen deze versies was de keuze van het vakgebied. In Focus II startten alle scholen met OGW voor rekenen-wiskunde, in Focus III kon ook gestart worden met OGW voor spelling, woordenschat of technisch lezen. Daarnaast omvatte Focus II één ronde van lesobservaties, terwijl dit er in Focus III twee waren.

\section{Onderzoeksvragen}

De Focus-interventie was erop gericht OGW in de deelnemende scholen te implementeren en verankeren, om zo het onderwijs beter te laten aansluiten op de onderwijsbehoeften van leerlingen, en daardoor de leerlingprestaties te verhogen. De hoofdvraag in het onderzoek naar de effectiviteit van de Focus-interventie was daarom: wat is het effect van de interventie op de leerlingprestaties in de deelnemende scholen?

Er zijn meerdere factoren waarvan wordt verondersteld dat deze samenhangen met de implementatie van OGW. In de Focus-training was daarom aandacht voor individuele factoren (LVS-kennis en onderwijskwaliteit), en factoren op schoolniveau (leiderschap, schoolcultuur en samenwerking). Voor elk van deze factoren is onderzocht hoe ze zich gedurende de interventie ontwikkelden, en in welke mate ze samenhangen met kenmerken op individueel niveau (zoals geslacht, leeftijd, functie en opleiding) in schoolniveau (zoals schoolgrootte, urbanisatie en SES).

Om de samenhang tussen deze factoren en de uiteindelijke effecten van de interventie te onderzoeken, is bovendien een exploratieve studie gedaan naar de verschillen tussen deze individuele en schoolorganisatorische factoren voor scholen met de grootste en scholen met de kleinste interventie-effecten op leerlingprestaties.

\section{Resultaten}

\section{Leerlingprestaties}

Om de effecten op leerlingprestaties te bepalen, is de prestatiegroei gedurende de twee jaar voor aanvang van de interventie vergeleken met de groei gedurende de interventie. Zowel voor Focus II als voor Focus III zijn de effecten op leerlingprestaties significant positief. Er is sprake van een extra leeropbrengst van gemiddeld ongeveer een maand gedurende de twee interventiejaren, voor het vakgebied waarmee is gestart (rekenen-wiskunde in Focus II, rekenen-wiskunde en spelling in Focus III).

Het effect dat we hebben gevonden op de leerlingprestaties kan worden beschouwd als substantieel. Uit eerder onderzoek blijkt dat het lastiger is om bij implementatie van een interventie onder grote groepen leerlingen een effect te vinden: hoe groter de groep, des te kleiner het effect. Als een interventie wordt geïmplementeerd onder 500 in plaats van 100 leerlingen, is het effect gemiddeld genomen slechts half zo groot. De effectgrootte wordt nogmaals gehalveerd bij een toename tot 3000 leerlingen. De Focus-interventie is geïmplementeerd onder maar liefst bijna 15.000 leerlingen (die dus gemiddeld 1 maand extra leerwinst realiseerden). Daarnaast is bij dit onderzoek gebruik gemaakt van onafhankelijke, gestandaardiseerde toetsen. Het is bekend dat effecten meestal groter zijn wanneer een test wordt gebruikt die door de onderzoekers is gemaakt.

Het effect op leerlingprestaties was niet voor alle scholen hetzelfde. Verschillen in effecten werden onder andere verklaard door de sociaal-economische status op leerling- en 
op schoolniveau (in zowel Focus II als Focus III was het effect groter voor leerlingen met een lagere SES en ook voor scholen met een groter aandeel gewichtenleerlingen) en het gekozen interventietraject (alleen in Focus III: in scholen waar langer dan één schooljaar aan OGW voor het betreffende vakgebied werd gewerkt was het effect groter).

\section{LVS-kennis}

LVS-kennis betreft de mate waarin leerkrachten, intern begeleiders en schoolleiders in staat zijn om de gegevens uit het leerlingvolgsysteem (LVS) correct te interpreteren. Bij aanvang en na afloop van de interventie hebben 1182 unieke deelnemers (Focus II en III) een kennistoets gemaakt.

Omdat door de deelnemende scholen drie verschillende computerprogramma's werden gebruikt voor de analyses van toetsgegevens, zijn drie versies van deze kennistoets ontwikkeld. Om de prestaties van alle deelnemers met elkaar te kunnen vergelijken, daarbij rekening te houden met de nesting van deelnemers binnen scholen, en om de effecten van de individuele kenmerken op voor- en nameting te kunnen schatten, is gebruik gemaakt van een multivariaat multilevel pre-post IRT-model.

Uit de resultaten blijkt dat de LVS-kennis van de Focus-deelnemers significant vooruit was gegaan, en ook dat er verschillen waren op basis van individuele kenmerken zoals functie en opleidingsniveau. Op de voormeting was nog sprake van een duidelijk verschil in prestaties tussen leerkrachten en schoolleiders, waarbij de laatste groep bij aanvang hoger scoorde, op de post-test was dit 'kennisgat' gedicht. Ook voor deelnemers met een hbo-opleiding versus deelnemers met een master-opleiding geldt dat een initieel verschil in kennisniveau werd opgeheven. Na afloop van de interventie waren de deelnemers dus beter in staat om tabellen en grafieken uit het LVS te interpreteren, en was het kennisniveau meer vergelijkbaar binnen de school. Het is aannemelijk dat dit bevorderlijk is voor het schoolbreed bespreken van de leerlingresultaten.

\section{Onderwijskwaliteit}

De laatste stap van de OGW-cyclus betreft het uitvoeren van de gemaakte plannen. De kwaliteit van de leerkracht is daarbij van groot belang. Om de onderwijskwaliteit in beeld te brengen, is gebruik gemaakt van leerlingvragenlijsten. Leerlingen zitten immers dag in, dag uit, bij hun leerkracht in de groep - dit in tegenstelling tot een externe observator die een enkele les komt observeren. Via vragenlijsten aan de leerlingen van groep 4 tot en met 8 is de kwaliteit van het onderwijs op vijf vlakken onderzocht: veilig klimaat, klassenmanagement, duidelijke instructie, het uitdagen van leerlingen, en doelgericht lesgeven. De eerste drie kwaliteitsaspecten worden beschouwd als basisvaardigheden, terwijl het uitdagen van leerlingen en doelgericht lesgeven worden gezien als meer specifieke OGW-vaardigheden.

Om de verandering tussen voor- en nameting te bepalen, en de effecten van leerling-, groeps- en leerkrachtkenmerken op deze tijdstippen afzonderlijk te kunnen modelleren, is gebruik gemaakt van een multivariaat, multilevel pre-post IRT-model.

De scores van 209 leerkrachten (allen Focus III) aan het begin van de interventie en na een jaar (aan het begin van het tweede interventiejaar) zijn met elkaar vergeleken. Hieruit bleek dat de basisvaardigheden van deze leerkrachten onveranderd waren, terwijl een significante toename van de OGW-vaardigheden werd vastgesteld. Voor de leerlingen was het dus duidelijker wat ze leerden, en waarom. Daarnaast monitorden leerkrachten de voortgang van leerlingen meer gedurende de lessen, en voelden zij zich meer uitgedaagd. Dit impliceert dat leerkrachten na afloop van de interventie ambitieuzere doelen stelden. 


\section{Opbrengstgericht Leiderschap}

De schoolleider wordt veeleal als cruciaal beschouwd voor de succesvolle implementatie van OGW. Aan het begin van de interventie, na afloop van het eerste jaar, en na afloop van het tweede jaar, is daarom op elke deelnemende school bij alle teamleden een vragenlijst afgenomen om de mate van opbrengstgericht leiderschap te bepalen.

Omdat alle teamleden op één school de vragenlijst invulden over dezelfde schoolleider, was een multilevel model noodzakelijk. Om de verschillende typen schoolleiders in kaart te brengen, is gebruik gemaakt van multilevel latente klassenanalyse. Vervolgens zijn de veranderingen gemodelleerd met behulp van multi-state modelling.

Op $44 \%$ van de scholen bleek de mate van opbrengstgericht leiderschap onveranderd. Dit waren met name scholen waarin bij aanvang al betrekkelijk hoog werd gescoord. Op 40\% van de scholen werd de schoolleider aan het eind van de interventie als meer opbrengstgericht beschouwd. Dit houdt in dat hij/zij bijvoorbeeld meer aandacht had voor wat er in de klassen gebeurde, leerkrachten beter ondersteunde bij OGW, en/of de gezamenlijke verantwoordelijkheid voor de leerlingprestaties meer benadrukte. Deze vooruitgang werd voornamelijk gevonden in scholen waarbinnen bij aanvang relatief laag werd gescoord. Op basis van deze resultaten kan gesteld worden dat opbrengstgericht leiderschap kan worden ontwikkeld door middel van een intensieve interventie, en dat de Focus-interventie op scholen met een aanvankelijk laag niveau van opbrengstgericht leiderschap heeft bijgedragen aan deze ontwikkeling.

\section{Opbrengstgerichte Schoolcultuur}

Een opbrengstgerichte cultuur wordt geacht bij te dragen aan de implementatie van opbrengstgericht werken. Hierbij valt te denken aan een gezamenlijke ambitie, hoge (maar realistische) prestatieverwachtingen, en het teambreed bespreken van leerlingprestaties.

Voor het bepalen van deze schoolcultuur is wederom multi-state modelling toegepast op de schoolcultuurklassen die bepaald werden door middel van multilevel latente klassenanalyse.

Scholen waarin de cultuur al opbrengstgericht was bij aanvang van de interventie, bleken in staat om deze cultuur vast te houden. In scholen waarbinnen de cultuur minder opbrengstgericht was, vond vaak een verandering naar een meer opbrengstgerichte cultuur plaats. Omdat het aannemelijk is dat de schoolleider een rol speelt in het ontwikkelen en behouden van een bepaalde schoolcultuur, is ook onderzocht wat de samenhang was tussen opbrengstgericht leiderschap en een opbrengstgerichte schoolcultuur. Uit deze analyses bleek dat er in scholen met een meer opbrengstgerichte schoolleider ook een meer opbrengstgerichte cultuur heerste.

\section{Samenwerking}

Opbrengstgericht werken is een teamaangelegenheid, samenwerking wordt daarom veelal gezien als essentieel voor de effectieve implementatie van OGW. Om inzicht te krijgen in (veranderingen in) samenwerkingspatronen binnen de deelnemende scholen, is gebruik gemaakt van sociale netwerkanalyse. Hierbij is het bespreken van respectievelijk de leerlingprestaties, de prestatiedoelen voor leerlingen en de instructiestrategieën onderzocht, door de dichtheid, de wederkerigheid en de centralisatie van de samenwerkingsnetwerken te bepalen. Uit de analyses blijkt dat het bespreken van de leerlingprestatiedoelen bij aanvang van de interventie minder gebruikelijk was, dan het bespreken van leerlingprestaties en instructiestrategieën. Het aantal wederkerige relaties was het hoogst voor het bespreken 
van leerlingprestaties, en het laagst voor het bespreken van de prestatiedoelen. De 'prestatienetwerken' waren gemiddeld het meest gecentraliseerd, wat erop kan wijzen dat een aantal invloedrijke teamleden hierbij centraal in het netwerk stonden. Dit zijn bijvoorbeeld de intern begeleiders, waarmee vrijwel elke leerkracht de leerlingprestaties bespreekt.

De netwerken met betrekking tot het bespreken van leerlingprestaties lieten een significante verandering zien. Enerzijds werden deze netwerken minder gecentraliseerd, anderzijds was het aantal wederkerige relaties op de nameting toegenomen. Wederkerige relaties worden vaak in verband gebracht met het overbrengen van meer complexe informatie. Deze bevindingen zouden er daarom op kunnen duiden dat leerkrachten na afloop van de interventie meer diepgaande gesprekken voerden over de leerlingprestaties.

\section{Verklaringen voor Differentiële Effecten}

Alle bovenstaande factoren (LVS-kennis, samenwerking, OGW-cultuur etc.) worden doorgaans verondersteld samen te hangen met de implementatie van OGW. De effecten van de interventie op de prestaties van leerlingen was niet voor alle scholen gelijk, en er werden eveneens grote verschillen vastgesteld tussen scholen met betrekking tot bovengenoemde factoren. De variatie tussen scholen met betrekking tot de factoren zou een verklaring kunnen bieden voor de verschillen tussen scholen qua interventie-effecten op leerlingprestaties. Om inzicht te verkrijgen in de relatie tussen die factoren en de effecten op leerlingprestaties, hebben we deze factoren vergeleken voor scholen met de grootste en scholen met de kleinste effecten op leerlingprestaties.

Uit deze verkennende studie bleek dat de scholen met een groot interventie-effect op leerprestaties zich van de scholen met een klein interventie-effect onderscheidden voor wat betreft de attitude van de school ten opzichte van OGW, de onderwijskwaliteit van de school, en de opbrengstgerichte schoolcultuur.

\section{Conclusie}

Ruim honderd scholen hebben deelgenomen aan het Focus-project. In deze training kwamen álle componenten van OGW op alle niveaus aan bod. Deze interventie onderscheidt zich van veel andere OGW-interventies, doordat het gehele schoolteam eraan deelnam.

Uit dit grootschalige onderzoek blijkt dat de leerlingprestaties, op scholen die aan het Focus-project hebben deelgenomen, tijdens de interventie hoger zijn dan vóór deelname aan het project. Daarnaast zien we dat de LVS-kennis, onderwijskwaliteit, opbrengstgericht leiderschap, schoolcultuur en samenwerking op de deelnemende scholen toenamen. De relatie tussen deze factoren en de effecten op leerlingprestaties is niet altijd eenduidig. Uit een overkoepelende vergelijking, bleek dat met name de initiële OGW-attitude, een opbrengstgerichte schoolcultuur en de onderwijskwaliteit een rol lijken te spelen voor het succesvol implementeren van OGW.

Naast de relevantie van dit onderzoek voor het terrein van OGW, heeft dit onderzoek ook bijgedragen aan de ontwikkeling en toepassing van geavanceerde statische methoden en technieken voor de analyse van het soort complexe data dat bij dit type schoolinterventies beschikbaar komt. 
Samenvatting (Dutch summary) 
From: Marieke

\title{
Dankwoord
}

To: Trynke

Subject: Dankwoord

Hela Trynke! We moeten ons dankwoord nog schrijven... dat moet wel een beetje goed zijn, want waarschijnlijk is dat het enige dat de meeste mensen lezen. Maar hoe begin je een dankwoord in een gezamenlijk proefschrift? Vaak zijn ze chronologisch, maar wat is 'het begin'? Was dat toen Adrie mij vroeg voor Focus II, of beginnen we bij jouw sollicitatiegesprek? En wie moeten we allemaal niet vergeten? Er zijn natuurlijk heel veel mensen die we allebei willen bedanken, maar hoe doen we dat met 'individuele bedankjes'? Heb jij een briljant idee?

\author{
From: Trynke \\ To: Marieke \\ RE: Dankwoord
}

Ha Marieke! Oehh goed punt, het dankwoord! Wat een gek idee dat we daar nu over moeten nadenken! Misschien moeten we wel iets zeggen over hoe het begon. Voor mij was dat het sollicitatiegesprek met jou en Adrie. Ik had de Research Master in Groningen bijna afgerond, en Roel Bosker tipte me over de vacature, dus schreef ik een brief en reisde ik af naar Enschede. Ik vond het project toen al heel erg leuk. Maar ja, die afstand!! Ik vond het (en nu nog steeds) zo moeilijk om Dokkum te verlaten, dat ik Adrie moest teleurstellen toen hij belde dat jullie voor mij hadden gekozen als tweede AIO op het project. Wat een geluk dat hij twee weken later nog een keer probeerde mij over te halen. Toen hij zei dat ik best in Dokkum kon blijven wonen, en zo nu en dan kon overnachten in Enschede, ging ik de uitdaging toch maar aan. Dat bleek uiteindelijk een hele goede keus!! :- Hoe begon jij eigenlijk bij Focus? En wie moeten we vooral niet vergeten te bedanken? Hadden we niet al eens ergens een lijstje gemaakt?

From: Marieke

To: Trynke

RE: Dankwoord

Haha, ja dat was me wat! We waren echt teleurgesteld toen je 'nee' zei. Het meisje dat we toen in jouw plaats hadden aangenomen haakte al snel weer af, en toen besloten we je gewoon nog eens te bellen. Ik had niet gedacht dat het zou lukken, maar je ging het toch proberen! Mijn start bij Focus was ook een beetje eigenaardig: ik had een sollicitatie lopen bij Cito, en vroeg Adrie of ik zijn naam mocht doorgeven toen ze naar referenties vroegen - hij was immers mijn afstudeerbegeleider. Tot mijn verbazing zei hij toen "als je toch op zoek bent, heb ik ook wel wat voor jou"! Daar hoefde ik niet lang over na te denken! In onze Dropbox (gaan we die nog een keer opruimen, of is dat inmiddels te laat?) vond ik na lang zoeken het bestand "Dankwoord - Wie moeten we niet vergeten", met een mooi lijstje. Zullen we er allebei een paar uitwerken? 
From: Trynke

To: Marieke

RE: Dankwoord

Ja! Dat lijstje herinner ik me nog, misschien moeten we er ook nog aan toevoegen: Dropbox, want als je samen een proefschrift schrijft is dat heel erg handig! (behalve die ene keer dat er op mysterieuze wijze allemaal bestanden waren verdwenen, maar gelukkig was dat in de literatuurmap en niet in ons 'PROEFSCHRIFT DEF' mapje). Moeten we in het dankwoord ook nog iets zeggen over hoe we uiteindelijk besloten dat het veel handiger was om samen een proefschrift te schrijven? Hebben we dat bij de ICO introductiecursus bedacht? Diana Dolmans gaf die cursus samen met Jeroen van Merriënboer, en zij maakte zich een beetje zorgen of het wel zo'n goed idee was. Samen een proefschrift schrijven brengt natuurlijk risico's met zich mee. We hebben nog samen met haar gebeld, besloten toch samen te werken, en natuurlijk waren we toen extra gemotiveerd om het te redden!

De ICO activiteiten vond ik sowieso een erg leuk onderdeel van het promoveren. Het was leuk om andere AIO's te leren kennen, en met elkaar over onze onderzoeken te praten. En tijdens de 'multi-level in SPSS'-cursus heeft Sven de Maeyer ons toch mooi op het R-spoor gezet. Zijn uitspraak "R heeft altijd gelijk" hebben we nog vaak tegen elkaar herhaald, als we weer eens een foutmelding kregen - en inderdaad, het lag altijd aan onszelf!

From: Marieke

To: Trynke

RE: Dankwoord

Volgens mij hebben we al heel snel besloten dat we een gezamenlijk proefschrift zouden schrijven, ik denk al vóór de introductiecursus, toen we het hadden over het verschil tussen de proefschriften die we zouden gaan schrijven. Omdat we concludeerden dat jij dan hetzelfde zou doen als ik, maar dan met nieuwe scholen, bedachten we volgens mij al snel dat we het interessanter vonden om dan alles gezamenlijk te doen- en zo ook meer te kunnen onderzoeken, Adrie vond het een prima plan.

Dat telefoongesprek met Diana was echt geweldig! We zaten toen samen op mijn kamer (oh ja, we zijn zelfs nog een tijdje geen kamergenoten geweest!) en zij stond op de speaker om haar zorgen te uiten. Op zich had ze wel een goed punt: dat we ook onze eigen keuzes moesten kunnen maken, dat we zo wel afhankelijk van elkaar werden, en vooral: dat we geen ruzie moesten maken omdat we dan echt een probleem zouden hebben. Maar wij waren vol vertrouwen, en besloten met haar advies in ons achterhoofd toch echt samen verder te gaan. Op elke ORD kwam ze even kijken bij onze presentaties, en vragen hoe het met de samenwerking ging, zo fijn dat we elk jaar konden zeggen 'hartstikke goed'. En nu werken we met haar en Jeroen samen in ons post-doc project!

Ik denk dat de belangrijkste mensen voor het slagen van ons project toch wel onze 
trainers zijn! Voor Focus II natuurlijk Bernard, Claudia, Janet en Wilma. En Marit, van Focus I, die ontzettend heeft geholpen met het inwerken van iedereen en heeft ingevallen wanneer nodig. Het leukst aan mijn trainers vond ik misschien wel dat ze zo verschillend waren, en dat ze daarom zo'n goed team waren bij het voorbereiden van de trainingen. Ook bij de scholen merkte ik, als ik eens meeging of een gesprek met een school had, dat ze allemaal goed gebruik maakten van hun eigen kwaliteiten.

From: Trynke

To: Marieke

RE: Dankwoord

Evelien, Hananja en Rob waren de trainers voor Focus III. Toen ze begonnen, hadden alle drie nog weinig ervaring met het geven van trainingen. Inmiddels zijn ze echte profs :-) En dat, terwijl we ze echt wel in het diepe gooiden! Ik denk nog steeds met heel veel plezier terug aan de Focus-dagen: dat alle trainers op de UT kwamen en dat we dan de hele dag bijeenkomsten aan het voorbereiden waren. Die dagen begonnen met de verhalen over de scholen en hoe de bijeenkomsten waren gegaan. Daarna moesten we hard aan het werk om de bijeenkomsten voor te bereiden. Het waren hectische dagen, en soms dacht iedereen: oei, komt dit wel goed? Maar het kwam altijd goed! De printers in de hele Cubicus waren op die dagen bezet omdat we nog van alles moesten printen.

Over die printers gesproken! In de eerste jaren van het project waren we beste vrienden met de mannen van de Xerox. Vooral Dennis was onze held, omdat hij soms last-minute toch nog de stapels met vragenlijsten, handboeken, of handouts voor de bijeenkomsten op tijd wist af te leveren, en op onmogelijke tijden nog storingen kwam oplossen of cartridges vervangen. Zijn telefoonnummer heeft heel lang met een hartje op ons whiteboard gestaan, hihi!

De print service valt een beetje in dezelfde categorie als de ICTS-service desk. Die staat ook op het lijstje van mensen die we niet moeten vergeten te bedanken, maar we hadden wel een beetje een haat-liefde verhouding met ze. We hebben heel vaak met Barry en Jan Jaap aan de telefoon gehangen als iets het weer niet deed. En gelukkig losten ze het uiteindelijk altijd weer voor ons op (bijvoorbeeld die ene keer dat je je hele dataset kwijt was vanwege een back-up fout, maar ze het daarna gelukkig terug vonden). Dus ook: dank aan de ICTS-service desk!

We waren wel goed in mensen voor ons aan het werk krijgen. De trainers, Dennis van de printer, de ICTS service desk, en ook flink wat student-assistenten. De eerste ronde vragenlijsten in Focus II hebben ze nog helemaal handmatig voor jou ingeklopt, en toen we daarna via Teleform de vragenlijsten konden scannen, was dat alsnog behoorlijk veel werk: scannen, checken, alles netjes archiveren. Ontzettend fijn dat we hen daarvoor konden inschakelen. De student die we in het bijzonder moeten bedanken is Dion, die ons heeft geholpen door alle papers taalkundig na te lopen. Zijn tips en correcties zijn heel waardevol geweest.

Ook de secretaresses hebben echt veel voor ons gedaan. Carola en Sandra hebben ons veel geholpen, met de werving van de scholen, het printen van certificaten 
en het beantwoorden van telefoontjes als wij weer eens op pad waren. En niet te vergeten Lorette en Birgit, zonder Lorette was het proefschrift nooit op tijd af geweest. Ik dacht dat ik veel van Word wist, maar zij is echt een Word expert!

From: Marieke

To: Trynke

RE: Dankwoord

"Weer eens op pad", dat was nogal vaak! Eerst de werving van de scholen, maar dat deden we samen met Adrie en vaak in zijn auto. Voor Focus II was dat midden in een strenge winter en zijn verwarming deed het niet. Hij zat in een jas, met een muts op en dikke wanten aan, en ik in een slaapzak in de auto. Bij de werving voor Focus III was dat toch minder ernstig. De intakegesprekken en alle andere activiteiten deden wij natuurlijk met de trein, en vaak namen we de OV-fiets voor het laatste stukje. Wat een top uitvinding!

Omdat jij in Dokkum woont, werkten we ook niet alleen samen in Enschede maar spraken we ook vaak af in Groningen of Zwolle. In Groningen werkten we dan op de RUG, meestal in het Harmoniegebouw. Bijna elke week wilde een afstudeerder ons wel een vragenlijst voor studenten laten invullen! De bibliotheek in Zwolle was ook zo'n fijne plek, jammer dat die ging verbouwen waardoor we er de laatste winter niet meer terecht konden, want we waren daar altijd erg productief.

Over productief gesproken: de schrijfweken vond ik ook echt fantastisch! We hadden echt een leuke groep AIO's, met Emmelien, Annemiek, Marjan en Marjoleine. Op de UT tijdens lunchwandelingen, koffiedates en vier-uur-dipjes, en met de schrijfweken als hoogtepunt. We kunnen er echt wel trots op zijn dat wij met $z$ 'n tweeën, maar ook met z'n allen, een goede balans hadden tussen hard werken en ontspannen. Zowel tijdens de schrijfweek als met bijvoorbeeld de ICO fall schools en conferenties lagen we tot's avonds laat te kletsen. We schakelden moeiteloos van korfbal, naar een presentatie, naar babies en trouwplannen.

Ik denk dat dat voor onze mannen ook wel grappig zal zijn geweest. Toen we na de AERA met $z$ 'n vieren die fantastische road trip om Lake Michigan maakten, hebben ze elkaar wel eens verwonderd aangekeken als wij bij een prachtige waterval ineens over een nieuwe invalshoek voor een paper begonnen!

From: Trynke

To: Marieke

RE: Dankwoord

Dit is wel een goed moment om jou ook te bedanken. Het is eigenlijk best heel bijzonder dat wij het zo goed met elkaar konden vinden! Volgens mij hebben we in die vier en een half jaar van onze promotie nooit ruzie gehad. We waren echt een goed team en vulden elkaar perfect aan. We hebben hetzelfde (wandel)tempo, houden van dezelfde kamertemperatuur en dezelfde snoepjes en koekjes. Als ik er even niet uitkwam, belde, mailde of 'lyncte' ik jou en dan kwam je altijd met een oplossing, of we klaagden samen even heel hard hoe zwaar we het hadden, om 
vervolgens weer vrolijk verder te gaan.

Niet alleen op werkgebied, maar ook privé kunnen we het heel erg goed met elkaar vinden en ik heb er echt een hele goede vriendin bij gekregen.

Onze begeleiders, Adrie en Jean-Paul hebben het denk ik best wel eens moeilijk gehad met het front dat wij vormden. Als wij een plan hadden, wat zij misschien toch niet zo'n goed idee vonden, was het moeilijk om dat uit ons hoofd te praten. Met de 'good cop/bad cop-methode' lukte het ons vaak toch ons plannetje erdoor te krijgen.

From: Marieke

To: Trynke

RE: Dankwoord

Ik ben het helemaal met je eens! Je hoort vaak dat een nadeel van promoveren is dat AIO's zich eenzaam voelen, maar daar heb ik nooit last van gehad: wij hadden elkaar! Dat kan inderdaad wel eens lastig zijn geweest voor Adrie en Jean-Paul. Tegelijk was het denk ik ook een voordeel: we bespraken alle overleggen samen voor, en stuurden stukken pas op als we er allebei naar gekeken hadden. En hoewel we inderdaad regelmatig tegen elkaar klaagden, hebben we ons ook vaak gelukkig geprijsd!

Adrie heeft altijd het volste vertrouwen in ons gehad, en nam ons en onze ideeën (hoe 'overdreven' hij ze soms ook vond) altijd serieus. We hebben vaak inhoudelijke discussies gehad, waarbij hij ook echt bereid was zijn mening te herzien als hij onze argumenten goed genoeg vond - in tegenstelling tot het stereotype beeld van de starre, dictatoriale professor waar je ook vaak AIO's over hoort klagen! Dat hij ons zo betrok bij zijn oratie vond ik echt een compliment, al is het voor het publiek ook maar goed dat hij zijn PowerPoints altijd even laat checken ;) Dat we dit soort opmerkingen ook in ons proefschrift zouden durven te publiceren, geeft wel aan dat we écht een goede, ook behoorlijk relaxte en informele band hebben met onze begeleiders!

Jean-Paul kwam er later bij als begeleider, omdat we verhuisden naar de vakgroep OMD. De statistiek vloog ons daar om de oren, maar dat vonden wij juist wel leuk. Onze echte, complexe datasets waren een mooie uitdaging, en Jean-Paul wilde die uitdaging met ons wel aangaan. Nou, dat hebben we geweten! Tijdens lange gesprekken waarin we er halverwege achter kwamen dat we volkomen langs elkaar heen praatten, of urenlange pruts-sessies met code in $\mathrm{R}$ of Mplus hebben we ontzettend veel geleerd. Jean-Paul had hoge verwachtingen van ons, en regelmatig dacht ik dat we die echt niet gingen waarmaken, maar wonder boven wonder lukte het meestal toch. Hij vond het geloof ik maar gek dat wij met z'n tweeën bij hem aan tafel zaten als hij het nieuwste programma wat hij voor één van ons had geschreven ging uitleggen. Maar als we elk de helft snapten, kwamen we er later samen altijd wel weer uit.

Grappige quotes van JP schreven we vaak op, ik vind dat er ten minste één in ons proefschrift moet, maar welke? Misschien dat hij die ene keer, toen we een 
voorstel deden voor een bepaald design zei: "Jullie zijn zó grappig. Ik ga nu even heel hard lachen op de gang".

Zullen we onze 'persoonlijke stukjes' ook naar elkaar sturen?

From: Trynke

To: Marieke

RE: Dankwoord

Ja, prima plan! Hierbij mijn stukje, een deel zul je wel niet helemaal begrijpen, want dat heb ik in het Frysk geschreven omdat ik eigenlijk vooral Dokkum wil bedanken! Ik was de afgelopen jaren heel veel van huis, maar vond het altijd fijn om weer thuis te komen. Mijn sociale leven daar, de korfballers, maar ook een paar mensen in het bijzonder.

In de eerste plaats Erik, die me zo support dat hij zelfs mee zou willen verhuizen naar Enschede, als dat nodig was. In tijden dat ik het even niet meer zag zitten, of te druk was met alle dingen die nog moesten, zorgde hij ervoor dat ik thuis weer tot rust kwam. Al was het alleen al, omdat hij altijd rustig is en zich nooit (te) druk maakt.

Heit en mem, foar it feilige thús, wêrtroch ik útdagingen oan doar te gean. Heit, dy't my de 'at it net kin sa as it moat, dan moat it mar sa as it kin' hâlding mei jûn hat, wêrtroch ik yn dit grutte projekt altyd wer in oplossing fûn foar de bearen op de dyk. Mem, mei wa't ik al myn ideeën en betinkingen oer it underwiis diele koe en sy my, mei al har ûnderfining as learkrêft op de basisskoalle, soms wer my beide fuoten op de grûn sette koe, troch sjen te litten dat in skoalle mear is as allinnich in plak wer't opbringsten wichtich binne.

Jurjen, dy't foar myn gefoel faker op my past, dan ik op him, al bin ik syn grutte sus.

Ta beslút, pake Keuning, dy't as haadmaster en yn 'e polityk in grut foarfjochter wie foar goed underwiis. Ea sei hy tsjin mei dat ik mar goed myn bêst dwaan moast op skoalle. Ik tink dat hy hiel grutsk wêze soe.

From: Marieke

To: Trynke

RE: Dankwoord

In veel proefschriften lees ik dankwoorden waarin mensen hun omgeving bedanken voor eindeloos geduld, begrip voor talloze afgezegde afspraken, en beloftes aan hun partner en gezin van de strekking 'nu dit klaar is, heb ik weer tijd voor jullie'. Daar herken ik mezelf helemaal niet in. Ik heb de afgelopen jaren met ontzettend veel plezier gewerkt aan dit onderzoek, maar daarnaast ook een heel leuk leven geleid, met genoeg ontspanning en aandacht voor wie me lief is. Een aantal van hen wil ik wel in het bijzonder even noemen, omdat zij mijn leven extra leuk maken. 
Jasper, je bent de beste. Om alles.

Mama, je hebt me geleerd altijd logisch na te denken en dingen van meerdere kanten te willen bekijken; de basisles voor een wetenschapper. Hoewel je keer op keer zei dat je er niks van begreep omdat het veel te ingewikkeld zou zijn, toonde je toch altijd interesse in mijn werk en liet je vooral merken dat je ontzettend trots op me bent en alle vertrouwen in me hebt.

Mirjam, ik ben blij dat jij, mijn grote kleine zusje, vandaag als paranimf achter me staat.

Oma, je bent zo ontzettend betrokken dat je elk krantenartikel over onderwijs voor me uitknipt en opstuurt. Hoewel ik dat niet altijd heb laten merken, vind ik dat ontzettend lief en ontroerend.

(ex-)WoesteLanders, het is altijd fijn met jullie. Van kampjes met dertig pubers naar vakanties met onze eigen geliefden en babies. Jaap, hoewel je momenteel in Australië woont, voel je nooit ver weg.

Buufs \& buurvrouw, onze spelletjes-, hardloop-, en BoerZoektVrouw-dates maken elk weekend een feestje. Ik ben blij dat ik bij jullie in de buurt woon.

Elja, je bent er nog maar net, maar nu al zo belangrijk. Ik kan me geen mooiere deadline voor ons proefschrift voorstellen.

From: Marieke

To: Trynke

RE: Dankwoord

Maar boven alles moeten we natuurlijk de scholen, besturen en alle leerlingen bedanken!

Beatrixschool, Haarlem

Bogermanschool, Rotterdam

de Bongerd, Oldenzaal

Bos en Vaart, Haarlem

de Brink, Lelystad

Catamaran, Rotterdam

de Cirkel. Haarlem

Crayenester, Heemstede

Dolfijn, Haarlem

Duinroos, Zandvoort

de Eemster, Dwingelo

Eduard van Beinum, Rotterdam

de Esch, Oldenzaal
From: Trynke

To: Marieke

RE: Dankwoord

Maar boven alles moeten we natuurlijk de scholen, besturen en alle leerlingen bedanken!

de Achtbaan, Moordrecht

Anne Frankschool, Bleiswijk

de Bonte Tol, Pijnacker

Da Costa, Den Haag

Drienermarke, Hengelo

DUO2002, Rotterdam

El Feth, Bergen op Zoom

Finnjol, Lelystad

Groene Hoek, Bergschenhoek

de Hoeksteen, Vlaardingen

Keijzerschool, Moerkapelle

de Klipper, Berkel en Rodenrij

de Meander, Delfgauw 
de Flambou, Gorredijk

Gerardus Majella, Vaassen

de Gorzen, Schiedam

Groene Palm, Rotterdam

Hannie Schaft, Zandvoort

Hannie Schaft, Haarlem

de Jasker, Nij Beets

't Kämpke, Lattrop

de Klinker, Schiedam

de Kring, Haarlem

de Krugerstee, Vaassen

Margrietschool, Halfweg

Meander, Lelystad

ML King. Haarlem

Molenwiek Dalton, Haarlem

't Oldhof, Borne

Peppelaer, Haarlem

Piramide, Haarlem

Prins Alexander, Rotterdam

Prins Alexander, Denekamp

Prinses Marijke, Den Haag

SamSam, Rotterdam

Sint Aegidius, Hertme

Spaarneschool, Spaarndam

Spitsbergen, Apeldoorn

Ter Cleeff, Haarlem

de Tjalk, Lelystad

Trimbeets, Gorredijk

Troubadour, Schiedam

Twickelo, Delden

de Veldkamp, Denekamp

de Vier Leeuwen, Rotterdam

de Voorsprong, Den Haag

Vrije School, Almelo

de Wheele, Borne

Wilgenhoek, Haarlem

Windroos, Deventer

de Wissel, Den Haag

de Wjukslach, Langezwaag

Zonnewijzer, Haarlem

Zuiderpolder, Haarlem

Zuidwester, Haarlem de Meerpaal, Scheveningen

't Meesterwerk, Schiedam

de Ontmoeting, Den Haag

Op Dreef, Zeist

de Optimist, Lelystad

Prinses Ireneschool, Den Haag

Prins Willem Alexander, Nieuwerkerk aan

den IJssel

de Poolster, Lelystad

de Regenboog, Schiedam

't Reigerbos, Zevenhuizen

Sint Bernardus, Schiedam

Sint Jan, Schiedam

Sint Jozefschool, Schiedam

Sint Willibrordus, Schiedam

de Sluis, Lelystad

het Startblok, Schiedam

Tarieq Ibnoe Ziyad, Eindhoven

de Terp, Nieuwerkerk aan den IJssel

de Triangel, Lelystad

de Tweemaster, Pijnacker

de Vlinder, Schiedam

het Visnet, Vlaardingen

Voorwegschool, Heemstede

de Vuurtoren, Den Haag

de Vuurtoren, Lelystad

Vuurvogel, Bergschenhoek

de Waterlelie, Rotterdam

de Winde, Nootdorp

Windroos, Lelystad

Zonnebloem, Deventer 
Dankwoord 


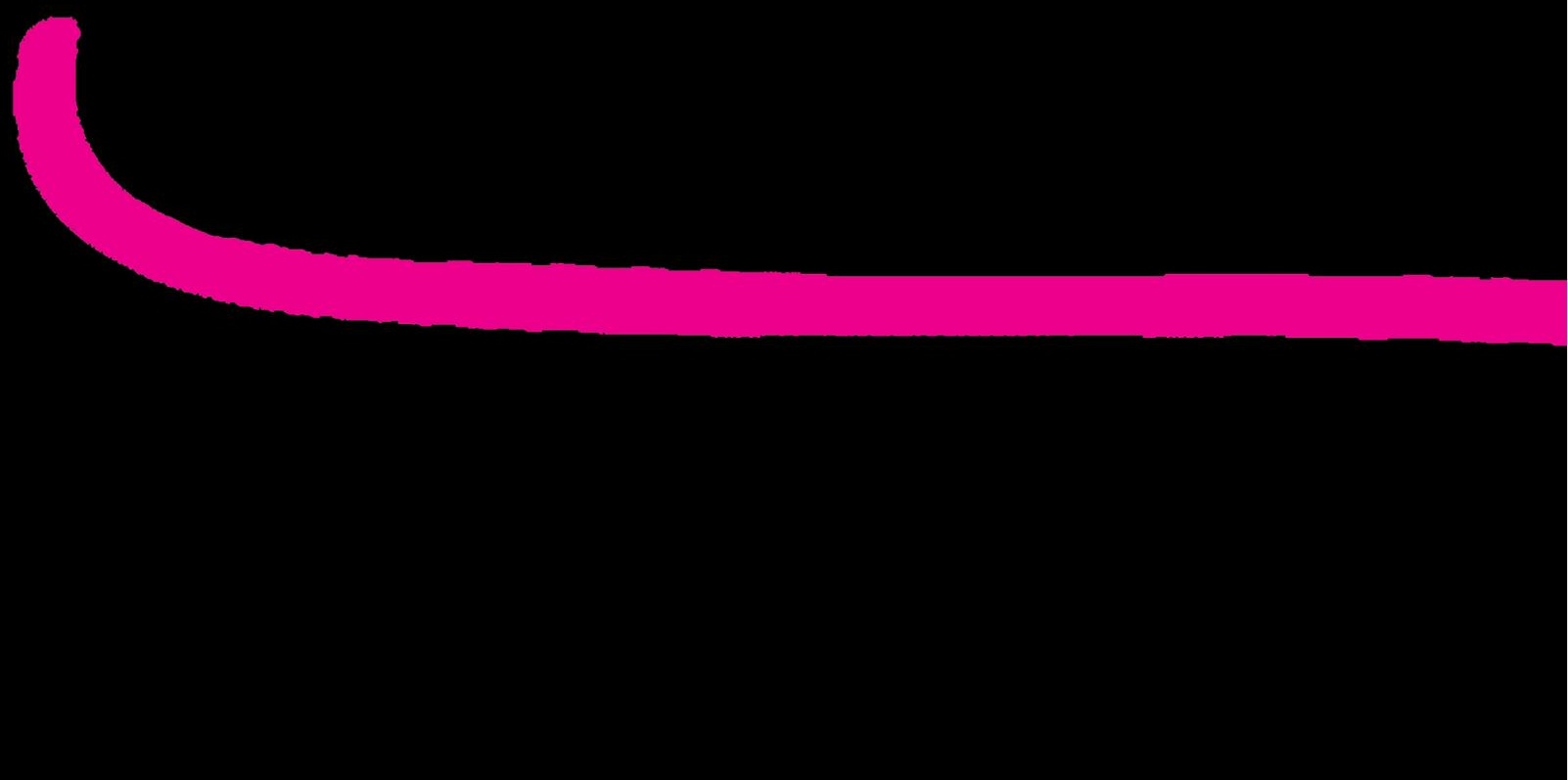

